\title{
SWEEP AND TAPER ANALYSIS OF SURFBOARD FINS USING COMPUTATIONAL FLUID DYNAMICS
}

\author{
A Thesis \\ presented to \\ the Faculty of California Polytechnic State University, \\ San Luis Obispo
}

\author{
In Partial Fulfillment \\ of the Requirements for the Degree \\ Master of Science in Aerospace Engineering
}

by

Brandon Baldovin

March 2019 
(C) 2019

Brandon Baldovin

ALL RIGHTS RESERVED 


\section{COMMITTEE MEMBERSHIP}

TITLE: Sweep and Taper Analysis of Surfboard Fins Using Computational Fluid Dynamics

AUTHOR: Brandon Baldovin

DATE SUBMITTED: March 2019

COMMITTEE CHAIR: David Marshall, Ph.D.

Professor of Aerospace Engineering

COMMITTEE MEMBER: Graham Doig, Ph.D.

Head Aerodynamicist, Canoo

COMMITTEE MEMBER: Aaron Drake, Ph.D.

Professor of Aerospace Engineering

COMMITTEE MEMBER: Hans Mayer, Ph.D.

Lecturer of Mechanical Engineering 


\begin{abstract}
Sweep and Taper Analysis of Surfboard Fins Using Computational Fluid Dynamics Brandon Baldovin
\end{abstract}

The research presented here provides a basis for understanding the hydrodynamics of surfboard fin geometries. While there have been select studies on fins, there has been little correlation to the shape of the fin and its corresponding hydrodynamic performance. This research analyzes how changing the planform shape of a surfboard fin effects its performance and flow field. This was done by isolating the taper and sweep distribution of a baseline geometry and varying each parameter individually whilst maintaining a constant span and surface area. The baseline surfboard fin was used as a template in Matlab to generate a set of $x$ and $y$ coordinates that defined the outline of the fin shape. These coordinates were then altered by changing either the sweep or taper distribution and resulted in new, unique planform shapes. The new shapes were used to generate 3D models with the NACA 0006 foil as the cross-section hydrofoil.

After the geometry was modeled, each fin was meshed and simulated in CFD for incidence angles ranging from $0^{\circ}$ to $20^{\circ}$ and a fin Reynolds number of $3.51 \times 10^{5}$. When the sweep distribution was changed, there was a direct correlation to vortex formation off the leading edge. Increasing the sweep generated a stronger vortex that persisted for higher angles of attack and resulted in higher moments but increased drag. Changing the taper distribution was not as influential. The tapered fin set showed similar flow fields and body forces to each other. Making a fin more rectangular had slight decreases in drag but made the shape more prone to separation. 


\section{ACKNOWLEDGMENTS}

I would like to thank Dr. Doig for being a major influence in my education and career. Without your relentless drive to bring out excellence in students, there is no doubt that I would not have applied myself and learned half of what I have. I would also like to thank Dr. Mayer for being the first positive role model I had as a professor early in my education. I am extremely grateful that you sat on my committee and showed me the power of a strong work ethic. I also thank Dr. Drake and Dr. Marshall for the expertise and professionalism in the field of aerospace engineering and fluid dynamics.

Thank you Tynan and Shaun for being incredible friends during grad school and Danny for almost watching Creed. You guys along with my fellow graduate and undergraduate students made my tenure at Cal Poly more than a fond memory but a major pillar of my life.

Eric, Adam, Abner, and Bryce, there is no way I could have pursued schooling for this long without your friendship. To my family, Mom, Dad, Danielle, Grandma, and Papa, this section is no where near long enough to show my appreciation. I could not have been raised in a better support system surrounded by loved ones.

Thank you Alex for being my partner in this journey. It has not been a short or easy one, but you have been there with me through all of, and nothing means more to me than that. 


\section{TABLE OF CONTENTS}

Page

LIST OF TABLES . . . . . . . . . . . . . . . . . . .

LIST OF FIGURES . . . . . . . . . . . . . . . . . . xi CHAPTER

1 Introduction and History . . . . . . . . . . . . . . . . 1

1.1 Surfing Background . . . . . . . . . . . . . . . . . 1

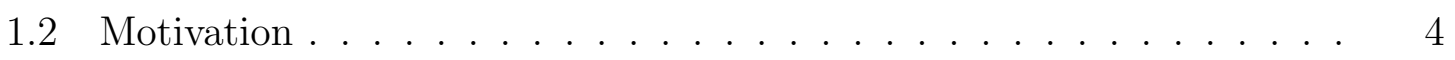

1.2.1 Turning Maneuvers ................ 5

1.3 Current Fin Configurations and Terminology . . . . . . . . . . . . 7

1.3.1 Naming Convention . . . . . . . . . . . . . . . . . 7

1.3.2 Fin Configurations ................. 9

1.4 Surfing Flow Field . . . . . . . . . . . . . . . . . . 11

1.4.1 Reynolds Number and Boundary Layers . . . . . . . . . . . . 14

1.5 Basic Fin Hydrodynamics . . . . . . . . . . . . . . . . . 17

1.5.1 Body Forces . . . . . . . . . . . . . . . . 17

1.5.2 Moments.................... 18

1.5.3 Vortex Shedding . . . . . . . . . . . . . . 19

1.6 Previous Work . . . . . . . . . . . . . . . . . 21

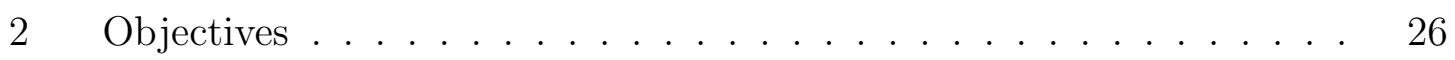

2.1 Problem Statement and Goal . . . . . . . . . . . . . 26

2.2 Methodology of this Study . . . . . . . . . . . . . 26

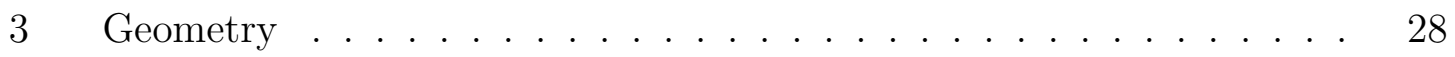

3.1 Hydrofoil Selection . . . . . . . . . . . . . . . . . 28

3.2 Fin Sweep Definition . . . . . . . . . . . . . . . . . 29

3.3 Fin Taper Definition . . . . . . . . . . . . . . . . . . . . . . . 31

4 Three Dimensional Modeling . . . . . . . . . . . . . 33

4.1 CAD Methodology . . . . . . . . . . . . . . 33

4.2 Constraints . . . . . . . . . . . . . . . . . . 33

4.3 Baseline Shape . . . . . . . . . . . . . . . . . 34 
4.3 .1 Image Processing . . . . . . . . . . . . . . . . . . . . . 35

4.4 Two Dimensional Point Manipulation . . . . . . . . . . . . . . . . 40

4.4 .1 Calculating Fin Sweep . . . . . . . . . . . . . . . . . 40

4.4 .2 Changing Fin Sweep . . . . . . . . . . . . . . . . . 44

4.4 .3 Calculating Fin Taper . . . . . . . . . . . . . . . . . 47

4.4.4 Changing Fin Taper . . . . . . . . . . . . . . . . . 48

4.5 CAD Creation . . . . . . . . . . . . . . . . . 53

5 Simulation Setup . . . . . . . . . . . . . 56

5.1 CFD Software and Resources _ . . . . . . . . . . . . 56

5.2 Physics Continua . . . . . . . . . . . . . . . . . 56

5.3 Fluid Domain . . . . . . . . . . . . . . . . . . . 58

$5.3 .1 \quad$ Fin Placement . . . . . . . . . . . . . . . . . . . . 60

$5.3 .2 \quad$ Fluid Domain Sizing . . . . . . . . . . . . . . . . . . 60

5.4 Mesh Generation . . . . . . . . . . . . . . . . . . 63

5.4 .1 Wall $\mathrm{Y}+\ldots \ldots \ldots \ldots \ldots \ldots \ldots$

5.4 .2 Prism Layers . . . . . . . . . . . . . . . . . . . . 64

5.4 .3 Mesh Independence . . . . . . . . . . . . . . . . . . . . . 66

5.5 Validation Test Case . . . . . . . . . . . . . . . . . . . 68

6 Results and Analysis . . . . . . . . . . . . . . . 71

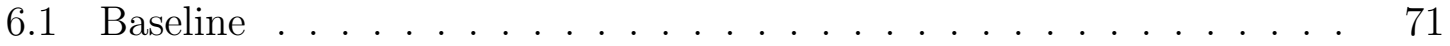

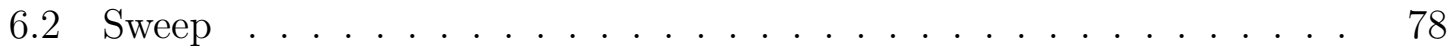

6.2 .1 Sweep Geometry . . . . . . . . . . . . . . . . . 78

6.2.2 $\Lambda_{\text {tip }}=15^{\circ}, 20^{\circ}, 25^{\circ}$ and $30^{\circ}$ Lift, Shear and Vorticity . . . . 79

6.2.3 $\Lambda_{\text {tip }}=40^{\circ}, 45^{\circ}, 50^{\circ}, 55^{\circ}$ Lift, Shear and Vorticity . . . . . . 83

6.2.4 Drag Comparison for Swept Fins _ . . . . . . . . . . 86

6.2.5 Moment Comparison for Swept Fins . . . . . . . . . . . . 88

6.3 Taper . . . . . . . . . . . . . . . . . . 90

6.3 .1 Taper Geometry . . . . . . . . . . . . . . . . . . . 90

6.3.2 Taper Lift, Shear, and Vorticity Comparison . . . . . . . . . 91

6.3.3 Taper Drag Comparison . . . . . . . . . . . . . . . . . . 95

6.3.4 Taper Moment Comparison _. . . . . . . . . . . . . . . 96

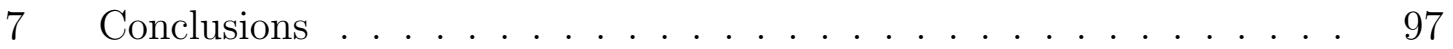


7.1 Effectiveness of Geometry Creation . . . . . . . . . . . . . 97

7.2 Effects of Sweep and Taper on Performance . . . . . . . . . . . 98

7.3 Future Work . . . . . . . . . . . . . . . . . . . . . . . . . . . . 99

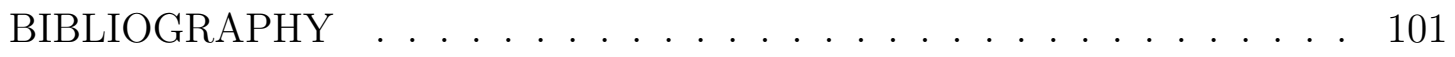
APPENDICES

A Tabulated Data . . . . . . . . . . . . . . . . . 105

A.1 Sampled Fin Measurements . . . . . . . . . . . . . . 105

A.2 Mesh Settings . . . . . . . . . . . . . . . . 106

B Fin Data . . . . . . . . . . . . . . . . . . . . . . 108

B.1 Baseline Fin . . . . . . . . . . . . . . . . . . . . . . . 108

B.2 Sweep . . . . . . . . . . . . . . . . . . 110

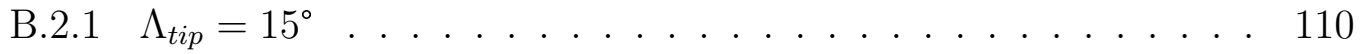

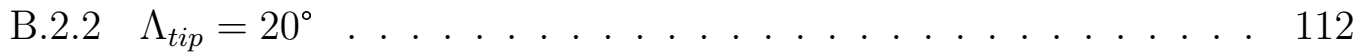

B.2.3 $\Lambda_{t i p}=25^{\circ} \ldots \ldots \ldots \ldots \ldots \ldots \ldots \ldots \ldots \ldots \ldots$

B.2.4 $\Lambda_{t i p}=30^{\circ} \ldots \ldots \ldots \ldots \ldots \ldots \ldots \ldots \ldots$

B.2.5 $\Lambda_{t i p}=40^{\circ} \ldots \ldots \ldots \ldots \ldots \ldots \ldots \ldots \ldots \ldots$

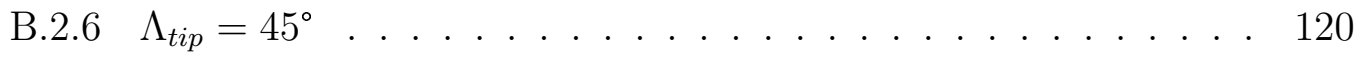

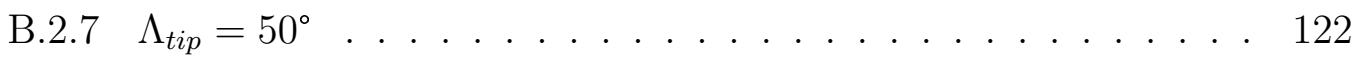

B.2.8 $\Lambda_{t i p}=55^{\circ} \ldots \ldots \ldots \ldots \ldots \ldots \ldots \ldots \ldots \ldots \ldots$

B.3 Taper . . . . . . . . . . . . . . . . . . . . . 126

B.3.1 $K_{\text {taper }}=0.50 \ldots \ldots \ldots \ldots \ldots \ldots \ldots \ldots \ldots \ldots$

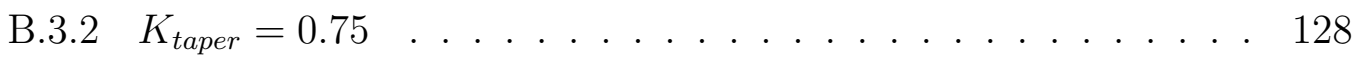

B.3.3 $K_{\text {taper }}=1.00 \ldots \ldots \ldots \ldots \ldots \ldots \ldots$

B.3.4 $K_{\text {taper }}=-0.50 \ldots \ldots \ldots \ldots \ldots \ldots \ldots \ldots \ldots \ldots$

B.3.5 $K_{\text {taper }}=-0.75 \ldots \ldots \ldots \ldots \ldots \ldots \ldots \ldots \ldots$

B.3.6 $K_{\text {taper }}=-1.00 \ldots \ldots \ldots \ldots \ldots \ldots \ldots \ldots \ldots$

B.4 Proportionally Tapered . . . . . . . . . . . . . . . . . . . . . . . 138

B.4.1 $K_{\text {taper }}=0.50 \ldots \ldots \ldots \ldots \ldots \ldots \ldots \ldots \ldots$

B.4.2 $K_{\text {taper }}=0.75 \ldots \ldots \ldots \ldots \ldots \ldots \ldots \ldots \ldots$

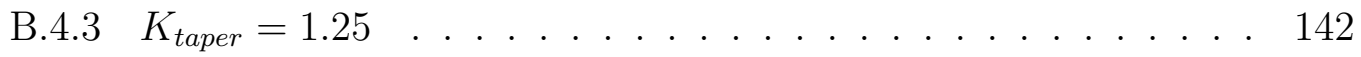

B.4.4 $K_{\text {taper }}=1.50 \ldots \ldots \ldots \ldots \ldots \ldots \ldots \ldots \ldots$

C Code ......................... 146 
C.1 Matlab Code . . . . . . . . . . . . . . . . . 146

C.1.1 Fin Planform Generation . . . . . . . . . . . . . . . . . 146 


\section{LIST OF TABLES}

Table

4.1 Baseline fin dimensions $[1] \ldots \ldots \ldots$. . . . . . . . . . . . .

4.2 Baseline fin dimensions after post processing. . . . . . . . . . . 39

4.3 Comparison of root chord and surface area for different tapered fins. $K_{\text {taper }}=0$ is the baseline geometry. . . . . . . . . . . . . 52

5.1 Mesh settings used for the domain sizing study. The sizes refer to the target cell size for the given setting. . . . . . . . . . . . . . . 60

5.2 Summary of the three domain sizes. The distances are an increments of $c_{r}=1.0 \mathrm{~m}$. . . . . . . . . . . . . . . . .

5.3 Results of the domain sizing study. Percent Difference is abbreviated as $\%$ Diff. . . . . . . . . . . . . . . . 62

5.4 Iterations of prism layer sizing on the surfboard fin. . . . . . . . 65

5.5 Iterations of prism layer sizing on the surfboard no-slip surface. . . 66

5.6 Summary of final mesh settings. The wake length refers to the distance of the wake refinement aft of the fin, and the wake density is the target cell size within the wake refinement. . . . . . . . . .

6.1 Lift curve slopes for high swept fin geometries. . . . . . . . . . .

A.1 Dimensions of surfboard fins. There is one outer fin. This is for the five fin setup, which uses a thruster configuration with the addition of two outer fins . . . . . . . . . . . . . . 106

A.2 Mesh sizing for the domain. The domain size represents the cell size of the volume mesh away from the surfboard fin, and the growth rate determines how fast the cells in the domain increase in size. . . . . 106

A.3 Mesh sizing for the wake behind the fin. The wake length represents the length of the refinement area aft of the fin, the wake angle determines the spread of the refinement, and the wake density determines the target cell size in the wake. . . . . . . . . . . . . . . . 107

A.4 Mesh sizing for the fin surface size. The fin surface size is the targeted mesh size on the fin surface and the minimum surface size is the allowed minimum size for cells wrapping around curvature. . . . . . 107 


\section{LIST OF FIGURES}

Figure

1.1 The first publicly, recognized surfboard fin, created and shaped by

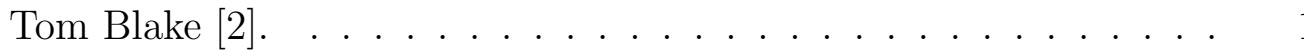

1.2 A keel fin (sometimes called a "D" fin). This particular fin was made

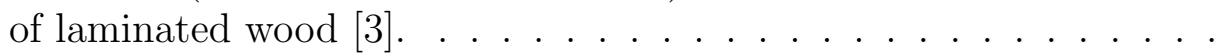

1.3 Comparison of a George Greenough's fin designed in 1960 versus a FCS fin made in 2018. Both share similar elongated and swept geometry. . . . . . . . . . . . . . . 3

1.4 A surfer at a wave pool in Waco, Texas. . . . . . . . . . . 4

1.5 A surfer performing a cutback maneuver on a wave. . . . . . . . . 5

1.6 A surfer performing an aerial maneuver. . . . . . . . . . . . . 6

1.7 Comparison of geometry naming conventions for surfboard fins and airplane wings. . . . . . . . . . . . . . . . . . . . . . 8

1.8 Labeled geometry parameters of a surfboard fin using aerospace no-

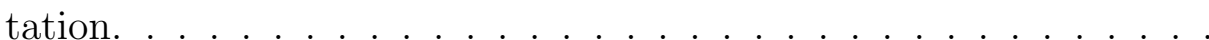

1.9 Illustration of a longboard fin versus a shortboard fin. A longboard fin will be used as the only fin of the surfboard while a shortboard fin will typically be used in a multiple fin configuration. . . . . . . . 10

1.10 Angles at which fins can be placed on a surfboard [4] . . . . . . . . 11

1.11 Sinusoidal structure for a set of waves [5] . . . . . . . . . . . 12

1.12 Sequence from left to right, top to bottom of a wave cresting and

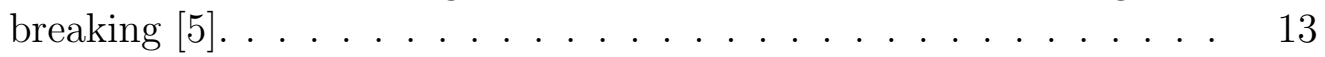

1.13 Cross sectional view of different surfboard bottom contours [6]. . . 14

1.14 Illustration of boundary layer growth and transition. The image depicts boundary layer growth over a flat plate, and the solid line

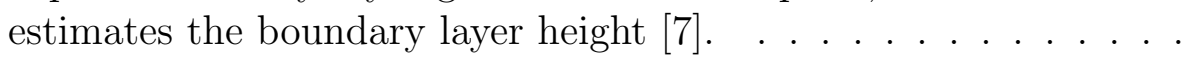

1.15 Horizontal cross section of root chord on fin, showing a sample hydrofoil, and a diagram of body forces with respect to a given freestream velocity vector. . . . . . . . . . . . . . . . 17

1.16 Diagram of the moment generated from the fin lift force. . . . . . . 19

1.17 The strong conical vortex can be seen in the right conditions on the Concorde as condensed water vapor [8]. . . . . . . . . . . . . . . 19

1.18 Underwater image of a surfboard with a thruster setup passing by. . 20 
1.19 (Left) Illustration of wind tunnel experiment setup. (Right) Picture of wind tunnel model $[9] \ldots \ldots$. . . . . . . . . . . . .

1.20 Flow visualization images of the tested keel fin at $R e=4.0 \times 10^{5}$. The left images are off the body of the fin using air bubbles and the right images are on the body using oil [4]. . . . . . . . . . . . . .

1.21 (Left) Fin Whale and (Right) Blue Shark fin's rescaled to a larger $\operatorname{span}[10] . \ldots \ldots \ldots \ldots \ldots \ldots$

$3.1 \quad$ NACA 0006 Airfoil. . . . . . . . . . . . . .

3.2 (a) Fin sweep defined from the root half-chord to the fin tip. (b) $\Lambda_{t i p}$ compared to the centerline of the fin. . . . . . . . . . . .

3.3 Sweep of the surfboard fin as a function of the span location. $\Lambda(y)$ is defined as the sweep at location $y$ from the root half chord to the centerline. . . . . . . . . . . . . . . . . .

3.4 (a) Rectangular wing taper. (b) Taper measurements for a surfboard fin, where $y$ is measured from $c_{r}$. . . . . . . . . . . . .

4.1 FCS II FT fin. The image shown is the center fin for the medium size fin set [1] . . . . . . . . . . . . . . . . . . . . 34

4.2 Edited image used to trace the edge of the baseline, planform shape. 36

4.3 Comparison of the edge coordinates after converting to units of meters and translating to an origin coordinate of $(0,0) \ldots . . . . .38$

4.4 Zoomed in window of edge coordinates. Because there are more than one point at a given $y$ coordinate, the points create a stair step like shape. . . . . . . . . . . . . . . . 39

4.5 Discrete point illustration of how $\Lambda(y)$ is defined. . . . . . . . . . . 40

4.6 Sweep distribution calculated using the centerline created from the baseline fin. . . . . . . . . . . . . . . . . . . 42

$4.7 \quad$ Filtered sweep data using a moving average filter. . . . . . . . . . . 42

4.8 Piecewise polynomial function fit to the baseline sweep distribution. 43

4.9 Planform outline for both the baseline shape and the new shape with changed sweep distribution. . . . . . . . . . . . . . . 45

4.10 Comparison of sweep distributions for $\Lambda_{t i p}=45^{\circ}$. . . . . . . . . 46

4.11 Comparison of Taper Distribution for $\Lambda_{t i p}=45^{\circ}$. The lines overlap, meaning the two distributions are equal. . . . . . . . . . . . 47

4.12 Taper distribution for baseline fin geometry along with a line illustrating the mean hydrodynamic chord. . . . . . . . . . . 
4.13 The leading and trailing edge overlapping before the fin tip. . . . .

4.14 Comparison of baseline shape with newly tapered fin after applying Equation 4.15 and $K_{\text {taper }}=0.75$. (a) Contains both the outline of the new shape along with the baseline fin. (b) The new fin outline.

4.15 Taper distribution comparison for $K_{\text {taper }}=0.75$. Note that the the distributions for both the new and baseline lines overlap at the MAC. 52

4.16 Sweep distribution comparison for $K_{\text {taper }}=0.75$. The red dashed line and black line perfectly overlap, illustrated equal sweep distributions. 53

4.17 Two view drawing of baseline fin with included isometric view. Units are in meters. . . . . . . . . . . . . . . . . . . . . 54

4.18 Zoomed in perspective of the fin tip hydrofoil used in the base geometry with a chord length of $10.41 \mathrm{~mm}$. The hydrofoil connected the leading and trailing edge at the tip. . . . . . . . . . . .

5.1 Two different boundary settings used depending on the incidence angle 59

5.2 Fluid domain bottom boundary split into three separate surfaces. The blue indicates the ocean surface where there is no boundary layer growth, and the grey indicates no-slip surfaces where the boundary layer is present. Freestream is going from left to right. . . . . . .

5.3 Three view drawing of the fluid domain. Grey is a no slip boundary, and the blue is for slip boundaries. All dimensions are in increments of $c_{r}=1.0 \mathrm{~m} . \ldots \ldots \ldots$. . . . . . . . . . . 62

5.4 Wall $y+$ for the baseline geometry at $\alpha_{F}=0^{\circ} \ldots \ldots 6$

5.5 Results of mesh sizing study. The numbers correspond to the iteration. 67

5.6 Comparison of $C_{L_{F}}$ for the Brandner and Walker test [4] and the Validation CFD at $R e=4.0 \times 10^{5}$. . . . . . . . . . . . . . . . 69

5.7 Comparison of $C_{M}$ for the Brandner and Walker test [4] and the Validation CFD at $R e=4.0 \times 10^{5}$. . . . . . . . . . . . . . . 70

5.8 Comparison of $C_{D}$ for the Brandner and Walker test [4] and the Validation CFD at $R e=4.0 \times 10^{5} \ldots$. . . . . . . . . . 70

6.1 Lift plot for the baseline fin configuration . . . . . . . . . 71

6.2 Lift distribution for the baseline fin configuration. . . . . . . . . . . 72

6.3 Skin friction coefficient comparison for the baseline fin configuration. 73

6.4 Streakline comparison for the baseline fin configuration. . . . . . . 74

6.5 Pressure versus shear drag for the baseline fin. . . . . . . . . . . 75 
6.6 Vorticity comparison for the baseline fin configuration. Each vorticity cross section is at $0.1 \mathrm{~m}$, and the fin surface has streakline visualization. . . . . . . . . . . . . . . 77

6.7 Planform shapes of swept geometries. . . . . . . . . . . 78

6.8 Lift curve comparison for $\Lambda_{\text {tip }}=15^{\circ}, 20^{\circ}, 25^{\circ}$, and $30^{\circ} \ldots \ldots$. . . 79

$6.9 \alpha_{F}=10^{\circ}$, skin friction coefficient comparison on the low pressure surface for the fins with decreasingly less sweep. Areas of white on the fin surface indicate high shear areas that exceed the maximum scalar value. . . . . . . . . . . . . . . . . . . 80

6.10 Vorticity images for $\Lambda_{t i p}=15^{\circ}$ through $30^{\circ}$ and the baseline geometry. 82

6.11 Lift curve comparison for $\Lambda_{\text {tip }}=40^{\circ}, 45^{\circ}, 50^{\circ}$, and $55^{\circ}$. . . . . . 83

$6.12 \alpha_{F}=10^{\circ}$, skin friction coefficient comparison on the low pressure surface for the fins with increasingly more sweep. . . . . . . . . . . 84

$6.13 \alpha_{F}=20^{\circ}$, skin friction coefficient comparison on the low pressure surface for the fins with increasingly more sweep. . . . . . . . . . . 84

$6.14 \alpha_{F}=20^{\circ}$, vorticity images for $\Lambda_{t i p}=40^{\circ}$ through $55^{\circ} \ldots \ldots$. . . . 85

6.15 Pressure versus shear drag comparison for all $\Lambda_{\text {tip }}$ values. The drag values are split into two plots: the lower and higher swept geometries. 87

6.16 Drag comparison for $\Lambda_{\text {tip }}=15^{\circ}$ and $55^{\circ}$ to the baseline geometry. . . 88

6.17 Drag comparison for $\Lambda_{\text {tip }}=30^{\circ}$ and $40^{\circ}$ to the baseline geometry. . . 88

6.18 Moment coefficient comparison for all $\Lambda_{\text {tip }}$ values. The drag values are split into two plots: the lower and higher swept geometries. . . 89

6.19 Planform shapes of tapered geometries. . . . . . . . . . . . . . . 90

6.20 Tapered fin lift curve comparison. . . . . . . . . . . . . . 91

6.21 Lift distribution comparison for $K_{\text {taper }}=0.75$ and -0.75 to the baseline geometry. Each line represents the lift distribution for a given $\alpha_{F}$ in increments of $5^{\circ} \ldots \ldots . \ldots . \ldots . \ldots . . \ldots 93$

6.22 Skin friction coefficient comparison on the low pressure surface for the fins with different taper distributions. Areas of white on the fin surface indicate high shear areas that exceed the maximum scalar value. . . . . . . . . . . . . . . . . . .

6.23 Vorticity comparison at $\alpha_{F}=20^{\circ}$ for positive $K_{\text {taper }}$ fins and the baseline geometry. . . . . . . . . . . . . . . . . . . 95

6.24 Pressure versus shear drag coefficient comparison for both positive and negative $K_{\text {taper }}$ fin geometries. . . . . . . . . . . . . . . . . 96

6.25 Moment coefficient comparison for each of the tapered geometries. $\quad 96$ 
B.1 Planform characteristics, baseline fin. . . . . . . . . . . . 108

B.2 Lift, drag, and moment coefficients, baseline fin. . . . . . . . . . 109

B.3 Vorticity cross sections on the suction surface, baseline fin. . . . . 109

B.4 $C_{f}$ coefficient contours on the suction surface, baseline fin. . . . . 109

B.5 Planform characteristics, $\Lambda_{t i p}=15^{\circ} \ldots \ldots \ldots \ldots \ldots \ldots$

B.6 Lift, drag, and moment coefficients, $\Lambda_{t i p}=15^{\circ} \ldots \ldots \ldots \ldots \ldots$

B.7 Vorticity cross sections on the suction surface, $\Lambda_{t i p}=15^{\circ} \ldots \ldots 111$

B.8 $C_{f}$ coefficient contours on the suction surface, $\Lambda_{t i p}=15^{\circ} \ldots \ldots 111$

B.9 Planform characteristics, $\Lambda_{t i p}=20^{\circ} \ldots \ldots \ldots \ldots \ldots \ldots \ldots$

B.10 Lift, drag, and moment coefficients, $\Lambda_{t i p}=20^{\circ} \ldots \ldots \ldots \ldots$

B.11 Vorticity cross sections on the suction surface, $\Lambda_{t i p}=20^{\circ} \ldots \ldots 113$

B.12 $C_{f}$ coefficient contours on the suction surface, $\Lambda_{t i p}=20^{\circ} \ldots \ldots 113$

B.13 Planform characteristics, $\Lambda_{t i p}=25^{\circ} \ldots \ldots \ldots \ldots \ldots \ldots \ldots$

B.14 Lift, drag, and moment coefficients, $\Lambda_{t i p}=25^{\circ} \ldots \ldots \ldots \ldots \ldots$

B.15 Vorticity cross sections on the suction surface, $\Lambda_{t i p}=25^{\circ} \ldots \ldots 115$

B.16 $C_{f}$ coefficient contours on the suction surface, $\Lambda_{t i p}=25^{\circ} \ldots \ldots 115$

B.17 Planform characteristics, $\Lambda_{t i p}=30^{\circ} \ldots \ldots \ldots \ldots \ldots \ldots$

B.18 Lift, drag, and moment coefficients, $\Lambda_{t i p}=30^{\circ} \ldots \ldots \ldots \ldots \ldots$

B.19 Vorticity cross sections on the suction surface, $\Lambda_{t i p}=30^{\circ} \ldots \ldots 117$

B.20 $C_{f}$ coefficient contours on the suction surface, $\Lambda_{t i p}=30^{\circ} \ldots \ldots 117$

B.21 Planform characteristics, $\Lambda_{t i p}=40^{\circ} \ldots \ldots \ldots \ldots \ldots \ldots$

B.22 Lift, drag, and moment coefficients, $\Lambda_{t i p}=40^{\circ} \ldots \ldots \ldots \ldots \ldots$

B.23 Vorticity cross sections on the suction surface, $\Lambda_{t i p}=40^{\circ} \ldots \ldots 119$

B.24 $C_{f}$ coefficient contours on the suction surface, $\Lambda_{t i p}=40^{\circ} \ldots \ldots \ldots$

B.25 Planform characteristics, $\Lambda_{t i p}=45^{\circ} \ldots \ldots \ldots \ldots \ldots \ldots$

B.26 Lift, drag, and moment coefficients, $\Lambda_{t i p}=45^{\circ} \ldots \ldots \ldots \ldots \ldots$

B.27 Vorticity cross sections on the suction surface, $\Lambda_{t i p}=45^{\circ} \ldots \ldots \ldots 121$

B.28 $C_{f}$ coefficient contours on the suction surface, $\Lambda_{t i p}=45^{\circ} \ldots \ldots \ldots 121$

B.29 Planform characteristics, $\Lambda_{t i p}=50^{\circ} \ldots \ldots \ldots \ldots \ldots \ldots \ldots \ldots$

B.30 Lift, drag, and moment coefficients, $\Lambda_{t i p}=50^{\circ} \ldots \ldots \ldots \ldots \ldots$

B.31 Vorticity cross sections on the suction surface, $\Lambda_{t i p}=50^{\circ} \ldots \ldots 123$

B.32 $C_{f}$ coefficient contours on the suction surface, $\Lambda_{t i p}=50^{\circ} \ldots \ldots 123$ 
B.33 Planform characteristics, $\Lambda_{t i p}=55^{\circ} \ldots \ldots \ldots \ldots \ldots \ldots \ldots$

B.34 Lift, drag, and moment coefficients, $\Lambda_{t i p}=55^{\circ} \ldots \ldots \ldots \ldots \ldots$

B.35 Vorticity cross sections on the suction surface, $\Lambda_{t i p}=55^{\circ} \ldots \ldots 125$

B.36 $C_{f}$ coefficient contours on the suction surface, $\Lambda_{t i p}=55^{\circ} \ldots \ldots 125$

B.37 Planform characteristics, $K_{\text {taper }}=0.50 \ldots \ldots \ldots \ldots \ldots$

B.38 Lift, drag, and moment coefficients, $K_{\text {taper }}=0.50 \ldots \ldots \ldots \ldots 127$

B.39 Vorticity cross sections on the suction surface, $K_{\text {taper }}=0.50 \ldots \ldots 127$

B.40 $C_{f}$ coefficient contours on the suction surface, $K_{\text {taper }}=0.50 \ldots \ldots 127$

B.41 Planform characteristics, $K_{\text {taper }}=0.75 \ldots \ldots \ldots \ldots \ldots$

B.42 Lift, drag, and moment coefficients, $K_{\text {taper }}=0.75 \ldots \ldots \ldots \ldots$

B.43 Vorticity cross sections on the suction surface, $K_{\text {taper }}=0.75 \ldots \ldots 129$

B.44 $C_{f}$ coefficient contours on the suction surface, $K_{\text {taper }}=0.75 \ldots \ldots$

B.45 Planform characteristics, $K_{\text {taper }}=1.00 \ldots \ldots \ldots \ldots \ldots$

B.46 Lift, drag, and moment coefficients, $K_{\text {taper }}=1.00 \ldots \ldots \ldots \ldots .131$

B.47 Vorticity cross sections on the suction surface, $K_{\text {taper }}=1.00 \ldots \ldots 131$

B.48 $C_{f}$ coefficient contours on the suction surface, $K_{\text {taper }}=1.00 \ldots \ldots .131$

B.49 Planform characteristics, $K_{\text {taper }}=-0.50 \ldots \ldots \ldots \ldots \ldots$

B.50 Lift, drag, and moment coefficients, $K_{\text {taper }}=-0.50 \ldots \ldots \ldots \ldots$

B.51 Vorticity cross sections on the suction surface, $K_{\text {taper }}=-0.50 \ldots 133$

B.52 $C_{f}$ coefficient contours on the suction surface, $K_{\text {taper }}=-0.50 \ldots 133$

B.53 Planform characteristics, $K_{\text {taper }}=-0.75 \ldots \ldots \ldots \ldots \ldots$

B.54 Lift, drag, and moment coefficients, $K_{\text {taper }}=-0.75 \ldots \ldots \ldots \ldots$

B.55 Vorticity cross sections on the suction surface, $K_{\text {taper }}=-0.75 \ldots 135$

B.56 $C_{f}$ coefficient contours on the suction surface, $K_{\text {taper }}=-0.75 \ldots 135$

B.57 Planform characteristics, $K_{\text {taper }}=1.00 \ldots \ldots \ldots \ldots \ldots$

B.58 Lift, drag, and moment coefficients, $K_{\text {taper }}=-1.00 \ldots \ldots \ldots \ldots$

B.59 Vorticity cross sections on the suction surface, $K_{\text {taper }}=-1.00 \ldots \ldots 137$

B.60 $C_{f}$ coefficient contours on the suction surface, $K_{\text {taper }}=-1.00 \ldots \ldots 137$

B.61 Planform characteristics, $K_{\text {taper }}=0.50 \ldots \ldots \ldots \ldots \ldots \ldots$

B.62 Lift, drag, and moment coefficients, $K_{\text {taper }}=0.50 \ldots \ldots \ldots \ldots$

B.63 Vorticity cross sections on the suction surface, $K_{\text {taper }}=0.50 \ldots \ldots 139$

B.64 $C_{f}$ coefficient contours on the suction surface, $K_{\text {taper }}=0.50 \ldots \ldots 139$ 
B.65 Planform characteristics, $K_{\text {taper }}=0.75 \ldots \ldots \ldots \ldots \ldots \ldots$

B.66 Lift, drag, and moment coefficients, $K_{\text {taper }}=0.75 \ldots \ldots \ldots \ldots$

B.67 Vorticity cross sections on the suction surface, $K_{\text {taper }}=0.75 \ldots \ldots 141$

B.68 $C_{f}$ coefficient contours on the suction surface, $K_{\text {taper }}=0.75 \ldots \ldots 141$

B.69 Planform characteristics, $K_{\text {taper }}=1.25 \ldots \ldots \ldots \ldots \ldots \ldots$

B.70 Lift, drag, and moment coefficients, $K_{\text {taper }}=1.25 \ldots \ldots \ldots \ldots$

B.71 Vorticity cross sections on the suction surface, $K_{\text {taper }}=1.25 \ldots \ldots 143$

B.72 $C_{f}$ coefficient contours on the suction surface, $K_{\text {taper }}=1.25 \ldots \ldots 143$

B.73 Planform characteristics, $K_{\text {taper }}=1.50 \ldots \ldots \ldots \ldots \ldots$

B.74 Lift, drag, and moment coefficients, $K_{\text {taper }}=1.50 \ldots \ldots \ldots \ldots$

B.75 Vorticity cross sections on the suction surface, $K_{\text {taper }}=1.50 \ldots \ldots 145$

B.76 $C_{f}$ coefficient contours on the suction surface, $K_{\text {taper }}=1.50 \ldots \ldots 145$ 
Chapter 1

\section{INTRODUCTION AND HISTORY}

\subsection{Surfing Background}

The origin of modern surfing dates to the early 1900's [12]. Craftsmen on Polynesian islands would shape tree trunks into long rectangular boards and would use these boards as a planing surface to ride an oncoming wave of water. Eventually, these boards would be named surfing boards or surfboards. As time progressed, surfing was discovered by western culture, and people looked to improve the speed of maneuverability of surfboards [13]. A breakthrough was made in 1934 when Tom Blake rode the first surfboard with an attached fin. The fin was a solid piece of timber attached to the bottom surface of the surfboard and would become instrumental to the performance of surfing [2].

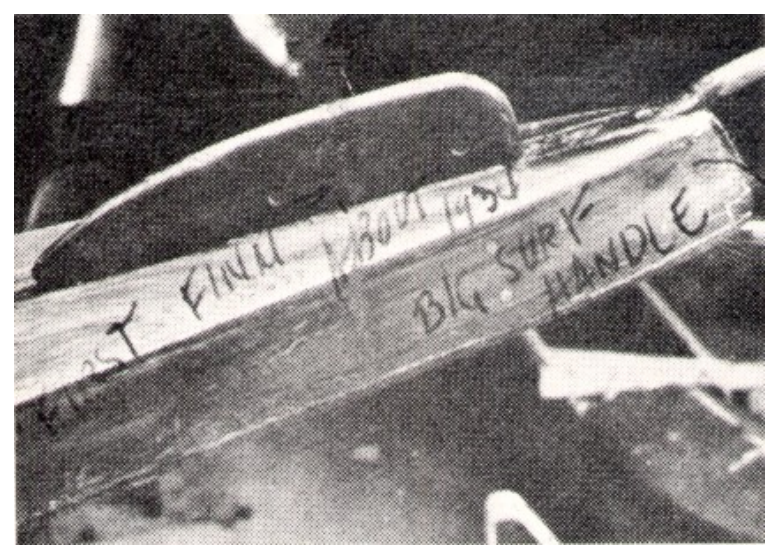

Figure 1.1: The first publicly, recognized surfboard fin, created and shaped by Tom Blake [2].

The fin became the first control surface for the surfboard. It was analogous to a tail fin on an airplane; it provided passive, directional stability and the capability for a controlled yawing motion. The tail fin on an airplane may have one or more hinged points for a rudder to move, but a surfboard fin has no degrees of freedom for 
rotation. Instead of any mechanical actuation to turn, the surfer will change their weight distribution above the fin, changing the moment arm and resulting in a turning motion.

Once it was understood that fins were instrumental to the surfer's stability (static or dynamic), the materials and shape used for creating them started to change. Up until 1960, wood wrapped in fiberglass was the primary choice for material, whilst maintaining a keel shape. The keel fin is the same shape seen in Tom Blake's fin and also in Figure 1.2. The leading edge holds an elliptical shape, the fin tip is rounded, and the trailing edge is straight.

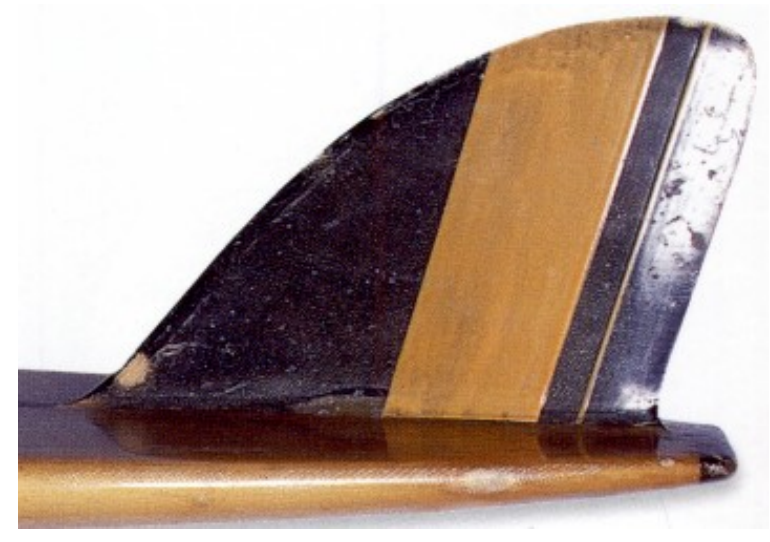

Figure 1.2: A keel fin (sometimes called a "D" fin). This particular fin was made of laminated wood [3].

After 1960, fin shapers began to use laminated fiberglass and started experimenting with different shapes and designs, and during this time period, George Greenough designed the first fin to have what is considered a modern shape, seen in Figure 1.3a.

Inspired by the tail fin of a tuna fish, Greenough used the curvature of the tail fin as motivation to design his now-famous fin shapes. Surfers felt an improvement using the higher aspect ratio fin, and soon, George Greenough's shape became the standard for surfboard fin designs. Eventually, this shape transitioned to mimicking the dorsal fin on a shark or dolphin as seen in Figure 1.3b. 

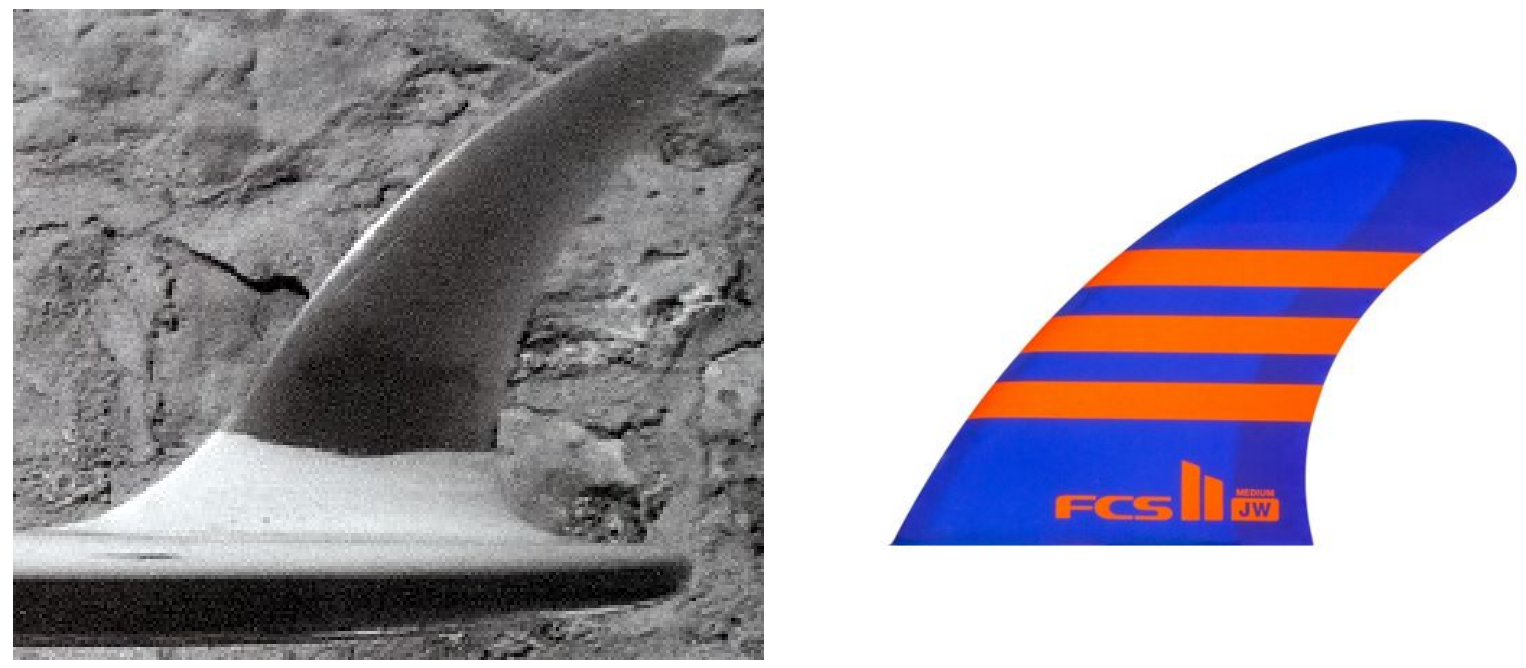

(a) George Greenough's first surfboard fin (b) FCS II Julian Wilson surfboard fin; the made in 1960 [14].

shape and design for this fin is considered typical for the modern era of surfing [15].

Figure 1.3: Comparison of a George Greenough's fin designed in 1960 versus a FCS fin made in 2018. Both share similar elongated and swept geometry.

The current state of fin shapes does not necessarily reflect an optimization of the fin's performance. The original concept for the modern surfboard fin was bio-inspired, and future shapers did not validate this shape with any quantitative analysis. Instead of testing the fin's shape in water tunnels or with computer simulations, shapers would use guess and check methods to prove their concepts. A new shape was made, a surfer used it, and then based on the feedback of whether or not the fin performed well from the surfer's opinion, the shaper would use or discard that shape [16].

Decades of this trial and error method have led to the current state of fin design and to a very subjective based grading of a surfboard fin's performance and quality. While there have been select studies that have analyzed environments with fins in them, there has yet to be a comprehensive look at the geometry of a surfboard fin. 


\subsection{Motivation}

Surfing as a sport has seen growth in the recent decade, with an increase of $50 \%$ of people who surf at least once a year and a forecasted 13 billion dollars net industry worth in 2017 [17]. Part of this growth stems from the emergence of wave pools. Wave pools are large ponds of water that generate waves mechanically. By either pulsating the water or creating an undertow with a moving hydrofoil, wave pools artificially create waves, and because this is a mechanically created wave, the waves are consistent every time [18]. This is not always the case when surfing in the ocean, where the change in tide, wind or sand bar underneath can change the surfing conditions hourly. For the first time, surfing now has a viable, even, playing field for all athletes and a new arena for competition. This has led to increased popularity and facilitated surfing in the Olympics for the very first time in 2020.

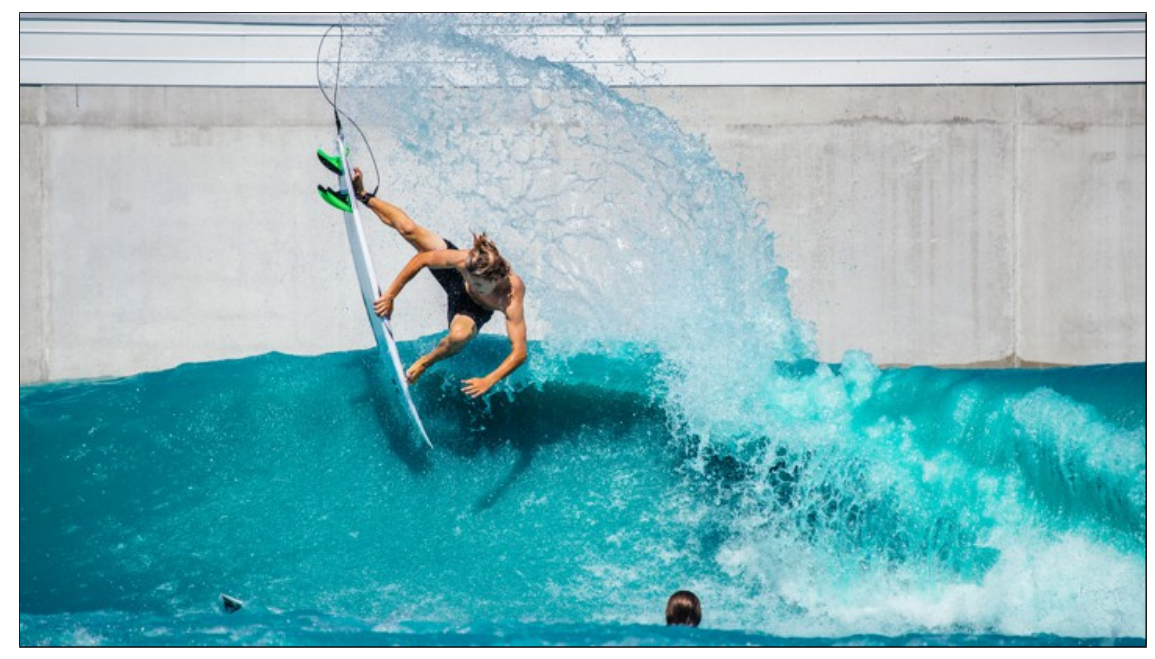

Figure 1.4: A surfer at a wave pool in Waco, Texas.

The combination of wave pools, the Olympics, and the increased accessibility of surfing have brought forth a new generation of athletes. These athletes need the best performing equipment possible to improve and compete. However, the shape design of surfing equipment does not represent a quantitative optimization derived from a 
set of requirements. The shaper tries to feel out what would be best and goes with their intuition.

This leaves room for improvement. If designers want to improve how they design and shape surfboard fins or surfing equipment, there needs to be an understanding of the fin hydrodynamics. While there is a wealth of knowledge on hydrodynamics and aerodynamics applied to wings, there is yet to be a thorough, reliable study that focuses on characterizing the shape of a surfboard fin. This was the primary source of motivation for this research.

\subsubsection{Turning Maneuvers}

The fin design and shape ultimately become important for how it maneuvers in the water. Three out of the five scoring categories in competitive surfing involve how the athlete maneuvers [19], so the fin's performance directly influences how well an athlete can score. A common maneuver is called a cutback and is where a surfer starts high on the wave face and turns sharply to face the other direction, seen in Figure 1.5.

Often the goal of the cutback is to spray as much water as possible and to turn

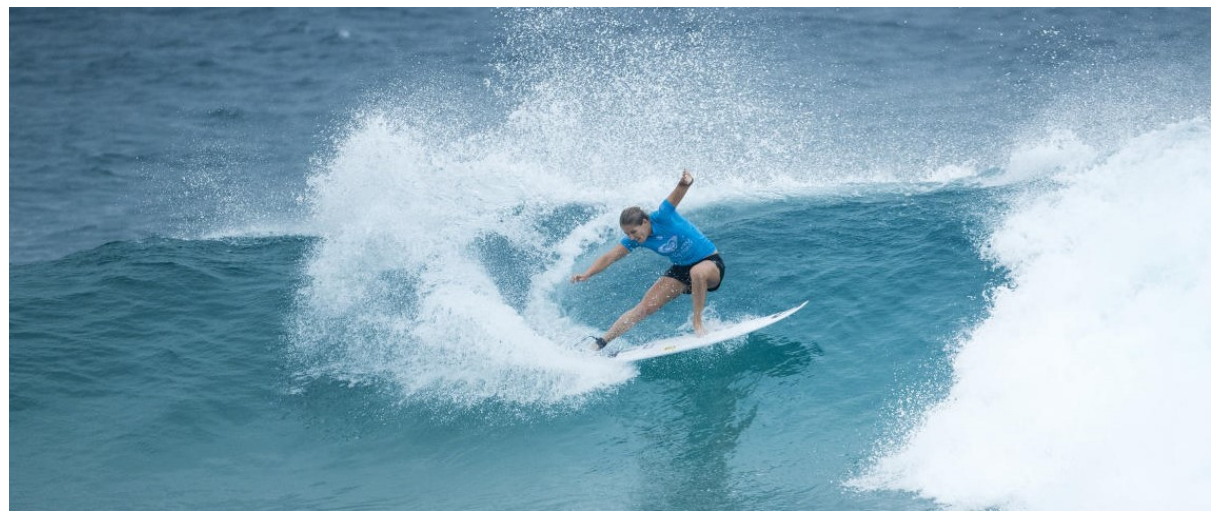

Figure 1.5: A surfer performing a cutback maneuver on a wave. ${ }^{1}$

\footnotetext{
${ }^{1}$ http://www.worldsurfleague.com/posts/stephanie-gilmore-the-surfers-surfer
} 
close enough to the lip of the wave that the fins become exposed. In doing so, the surfer shows power and mastery of the maneuver.

Turning quickly and efficiently in the water takes effort from the surfer, but is made easier by how much fin lift and moment the fins generate. The surfer also loses a lot of speed after performing a cutback, so having low drag is important to re-accelerate back out in front of breaking water.

There are also surfer's who accelerate fast enough to launch off the lip of a wave, hang in the air, and land back on the same wave. This is called an aerial maneuver, and a variation of this is seen in Figure 1.6.

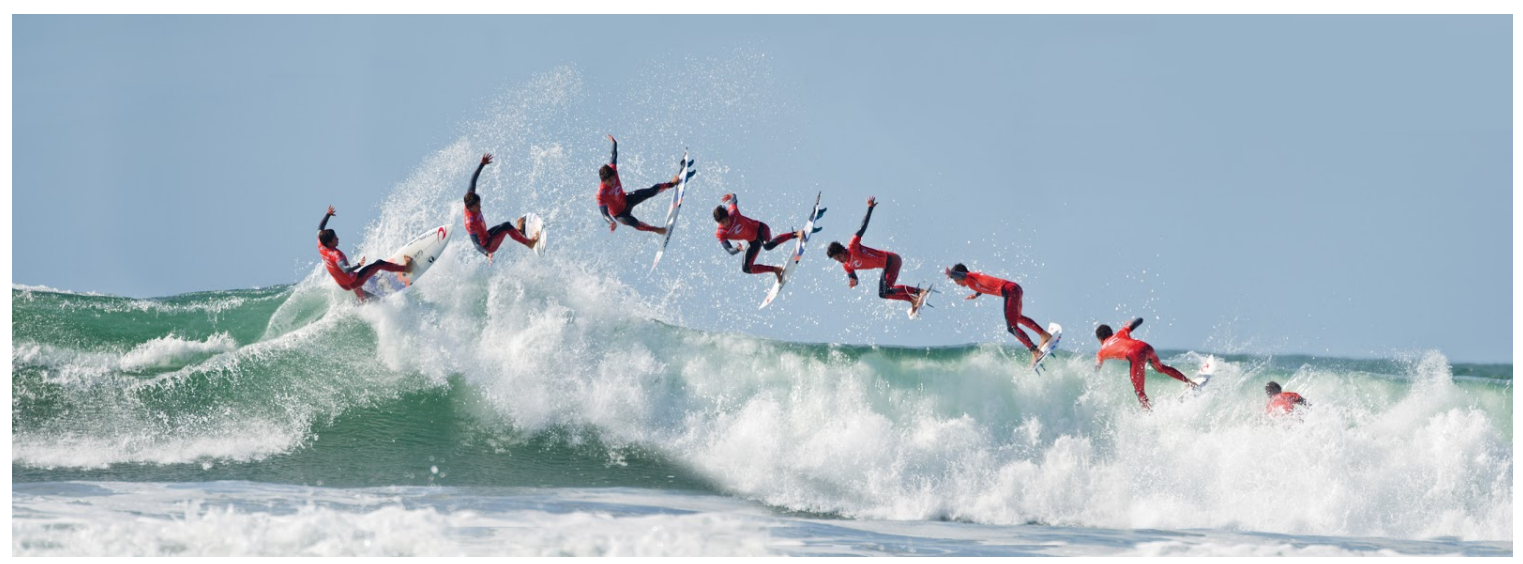

Figure 1.6: A surfer performing an aerial maneuver. ${ }^{2}$

In order to perform an aerial, the surfer has to maintain high speed and stay efficient with their movements. This implies that the fin lift to drag ratio needs to be high, and there should not be any early separation across the fins. Separation would decelerate the surfer from the large pressure drag created, so the fins need to delay the separation angle as long as possible.

There are a variety of other maneuvers that surfer's can perform, but the cutback and aerial are two of the most common. Performing them becomes more difficult

\footnotetext{
${ }^{2}$ https://www.theinertia.com/disruptors/christian-fletcher-wins-1989-surf-bout-opens-door-foraerial-surfing/
} 
from poorly designed equipment, so it is important that the fins used are optimized for a given environment and set of maneuvering requirements.

\subsection{Current Fin Configurations and Terminology}

A surfboard fin has a unique set of parameters and environmental conditions that define it's application and use. While a lot of concepts are similar to other aerodynamic or hydrodynamic applications, there are a few clarifications that must be made before moving into the analysis of the fin.

\subsubsection{Naming Convention}

A surfboard fin's geometric parameters are similar to an airplane wing or rudder with a few discrepancies. Meaning, the reference lengths on a surfboard fin are the same to that of an airplane wing, but the names used vary. Figure 1.7 presents a comparison of the naming convention.

Instead of defining a chord length on a fin, it's width will be described by how long the base is, and sometimes the sweep of the fin will be described as the rake. An issue with some of the terminology associated with fins is that there is inconsistency in the surfing community with how each parameter is explicitly defined. An example of this is referenced from two companies describing the starting and ending points of the rake differently $[21,22]$. One describes the rake as the curvature and length of the leading edge, and the other as an angle from root chord to tip. This can lead to some confusion as to what parameters correspond to. For this reason, the naming convention and definitions of an airplane wing will be used in this paper.

The goal was then to define the aspect ratio of the fin to be the same as an airplane wing. The leading edge of the surfboard fin is defined as the edge length of 


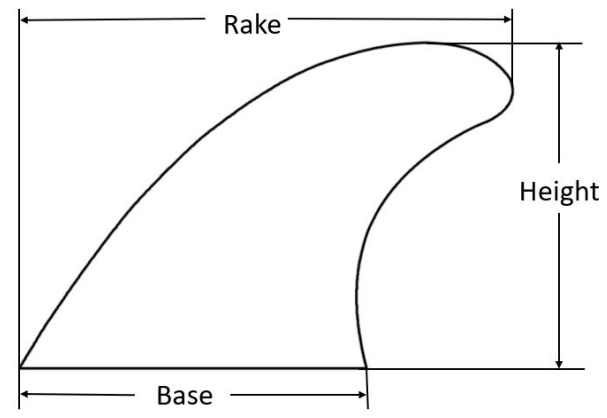

(a) Geometric parameters on a fin.

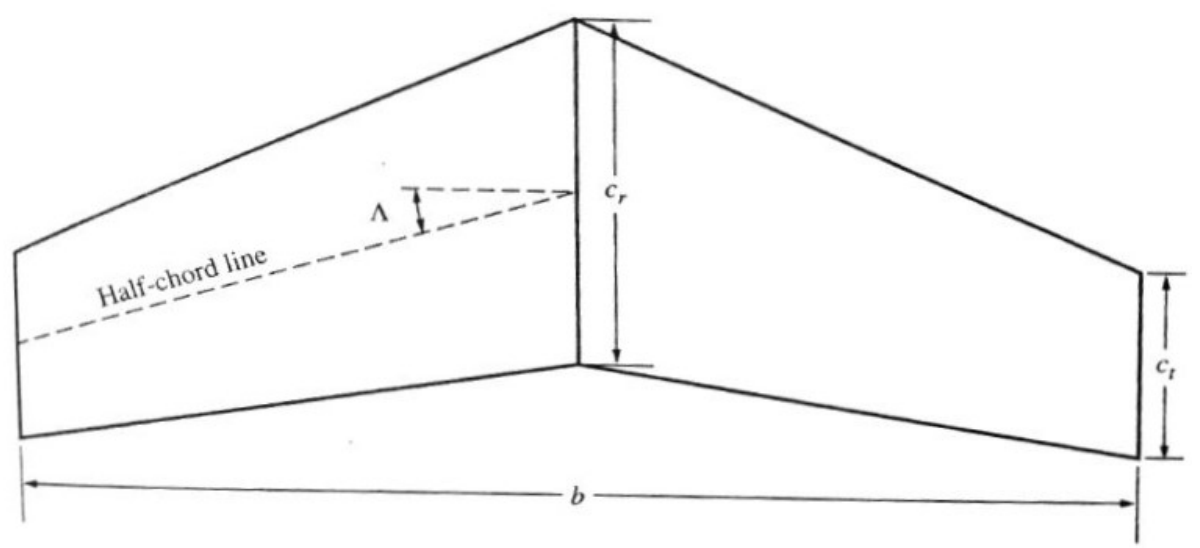

(b) Geometric parameters on an airplane wing. $\Lambda$ is the sweep of the wing, $b$ is the wing span, and $c_{r}$ and $c_{t}$ are the root and tip chords respectively [20].

Figure 1.7: Comparison of geometry naming conventions for surfboard fins and airplane wings.

the fin that encounters freestream conditions, $U_{\infty}$, first, and the trailing edge is the opposite edge (the freestream velocity is the velocity upstream of an object before it's perturbed). The length at a given cross section from the leading edge to the trailing edge is described as the chord length, $c$, of the fin, and the chord length at the root of the fin, the base length in Figure 1.7, is the root chord, $c_{r}$. The span of the fin, $b$, is defined as the height, or the orthogonal length from root chord to the tip of the fin. This leads to defining the aspect ratio of the fin the same as an airplane wing, Equation 1.1, where $S$ is the projected area of the fin. 


$$
A R=\frac{b^{2}}{S}
$$

When the aspect ratio of a wing changes, it influences the proportionality of induced and parasitic drag, along with the wing loading [8]. Induced drag primarily stems from the presence of vortices, and parasitic drag is the friction portion of drag in a fluid; both are defined in following sections. Changing the aspect ratio can also change the mean hydrodynamic chord, $M H C$, which is the average chord length of the fin. The $M H C$ is often used as the reference length for calculations.

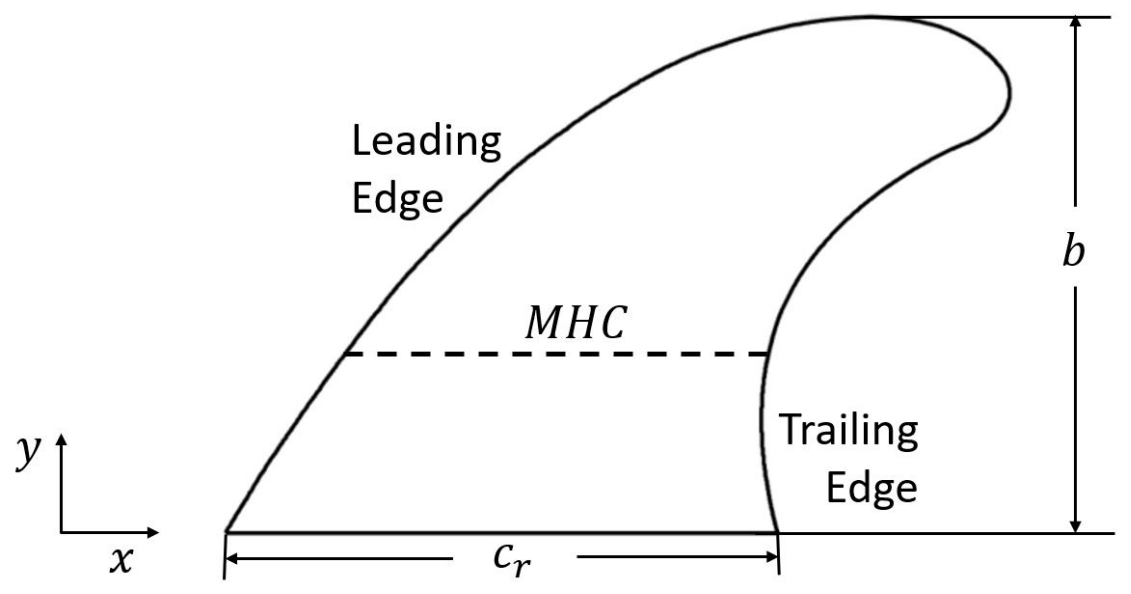

Figure 1.8: Labeled geometry parameters of a surfboard fin using aerospace notation.

\subsubsection{Fin Configurations}

There are two prominent styles of surfboard fins, and they reflect the type of board

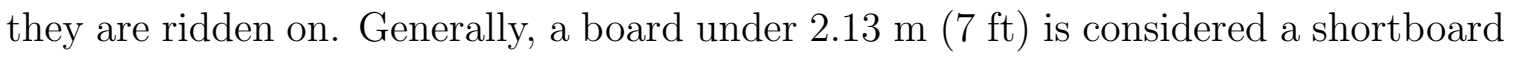
and will use multiple lower aspect ratio fins with the same dorsal fin shape described previously. Surfboards larger than that are classified as longboards and will use a single, larger, high aspect ratio fin.

Longboards are heavier than shortboards, so the size of the fin is typically larger and longer to produce a bigger moment for the surfer to turn the board. Surfers who 
ride longboards don't use multiple fin systems as depicted in Figure 1.9, not because the single fin performs better on a longer board, but because surfer's use long boards for smoother surfing. A long board is not meant for high performance maneuvering; it's for either beginner surfers or surfing on smaller waves. This distinction is made because the single fin on a long board is an anomaly for fin shapes and designs.

Shortboards will use fin configurations with two or more fins on the bottom of the surfboard. The two fin configuration, or twin fin, is similar to the single fin in that it is used recreationally, not competitively. The most common shortboard configuration is the three fin setup, otherwise known as the thruster configuration. The thruster fin will have a symmetric fin that sits on the centerline of the board, and two asymmetric side fins. The twin fin and four fin, or quad, configurations, will have only asymmetric fins that sit off the centerline of the board.

There have been limited studies comparing the different configurations, but one has stated that thruster setups can produce more side force at lower angles of attack,

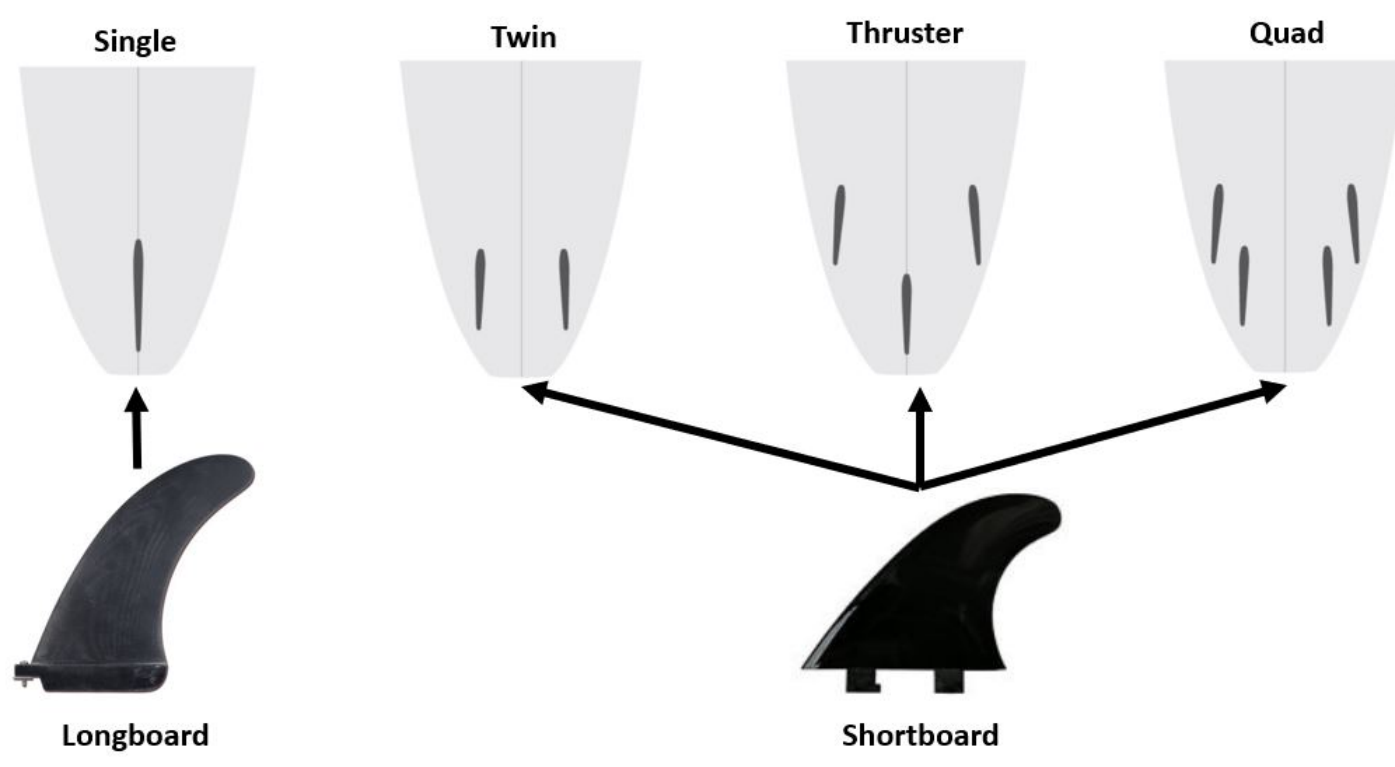

Figure 1.9: Illustration of a longboard fin versus a shortboard fin. A longboard fin will be used as the only fin of the surfboard while a shortboard fin will typically be used in a multiple fin configuration. 
increasing maneuverability [23]. A four fin configuration, or quad configuration, has more surface area and is better for holding a direction or trajectory on a wave [24]. The choice between using a quad or thruster will be the personal preference of the surfer, but more often than not, both professional and amateur surfer's will use thruster configuration.

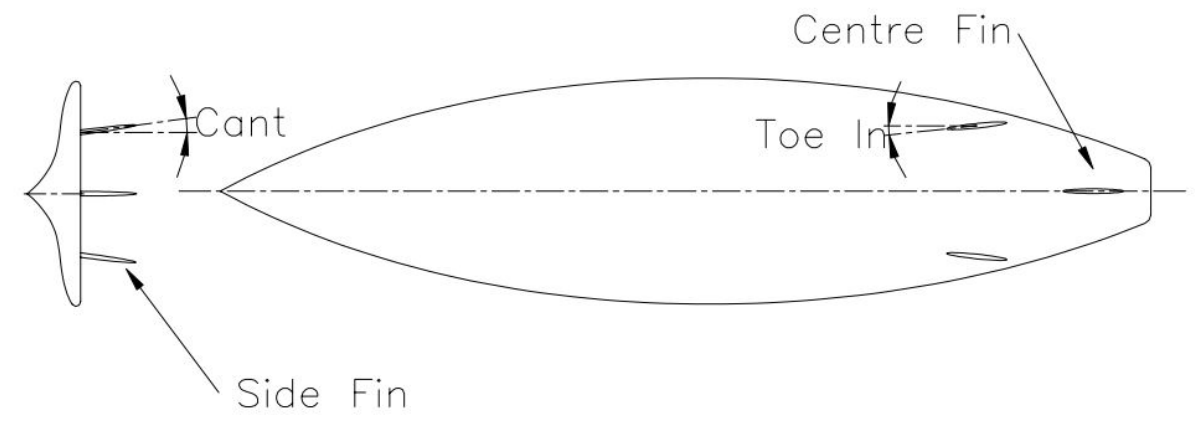

\section{Figure 1.10: Angles at which fins can be placed on a surfboard [4].}

The placement of fins on surfboards can be variable as well, as seen in Figure 1.10. The toe in angle is the angle at which a surfboard fin points at with respect to the centerline of the surfboard (i.e. a $0^{\circ}$ toe angle means that the surfboard fin is perfectly parallel with the length of the surfboard), and the cant is the angle that the side fins are tilted with respect to the center fin.

This research isolated a single, symmetric shortboard fin without the influence of side fins. This was to establish an understanding of the baseline performance of the fin shape, so that later, more precise design decisions can be made for different fin configurations.

\subsection{Surfing Flow Field}

One reason for small study numbers on surfboard fins comes from the difficulty in simulating the environment. As mentioned, surfing is a sport where a person uses 
a planing surface, called a surfboard, to ride breaking waves. The goal is to stay in front of the breaking water and ride the smoother part of the wave. This can be done in rivers or lakes, but is most commonly seen in the ocean.

The origin of a wave starts with shear and turbulent interactions of wind across the ocean surface that create small peaks and troughs. These are then amplified from the pressure gradient of the wind moving across the ocean surface and results in a group or set of waves [5]. The final structure is sinusoidal and can be visualized in Figure 1.11. Each peak also has a rotational component to it's motion. If the set of water is moving from left to right, as in Figure 1.11, there is a clockwise rotation as it moves past a given location.

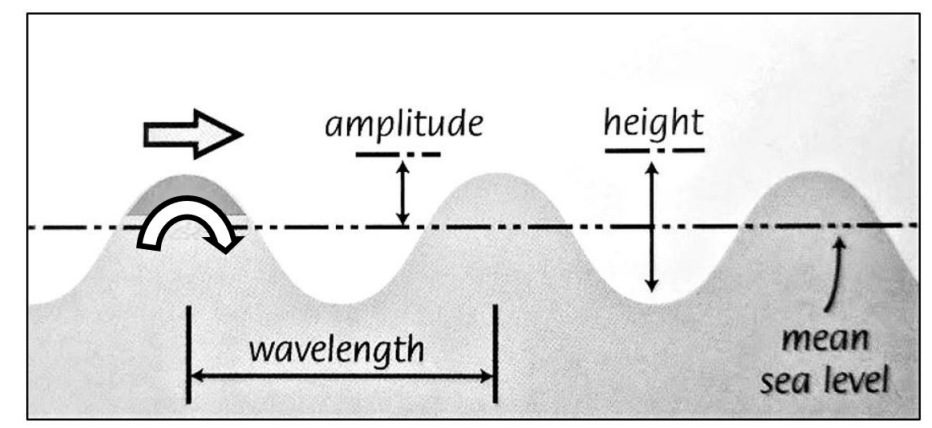

Figure 1.11: Sinusoidal structure for a set of waves [5].

As a set of waves approaches a body of land, the water becomes physically constrained by the ocean floor, either a reef or sand bar. The trough of the wave can no longer move as far down and is instead displaced away from the ocean floor. Due to the decrease in area, the water accelerates underneath and rotates towards the peak, and the wave crests, curls over and crashes. An illustration of this sequence can be seen in Figure 1.12.

This is a simplistic description of the motion and formation of waves. Often there are multiple periods for a group of waves interacting with each other, the shape of the ocean floor is variable, and the weather is highly influential. The combination of these 

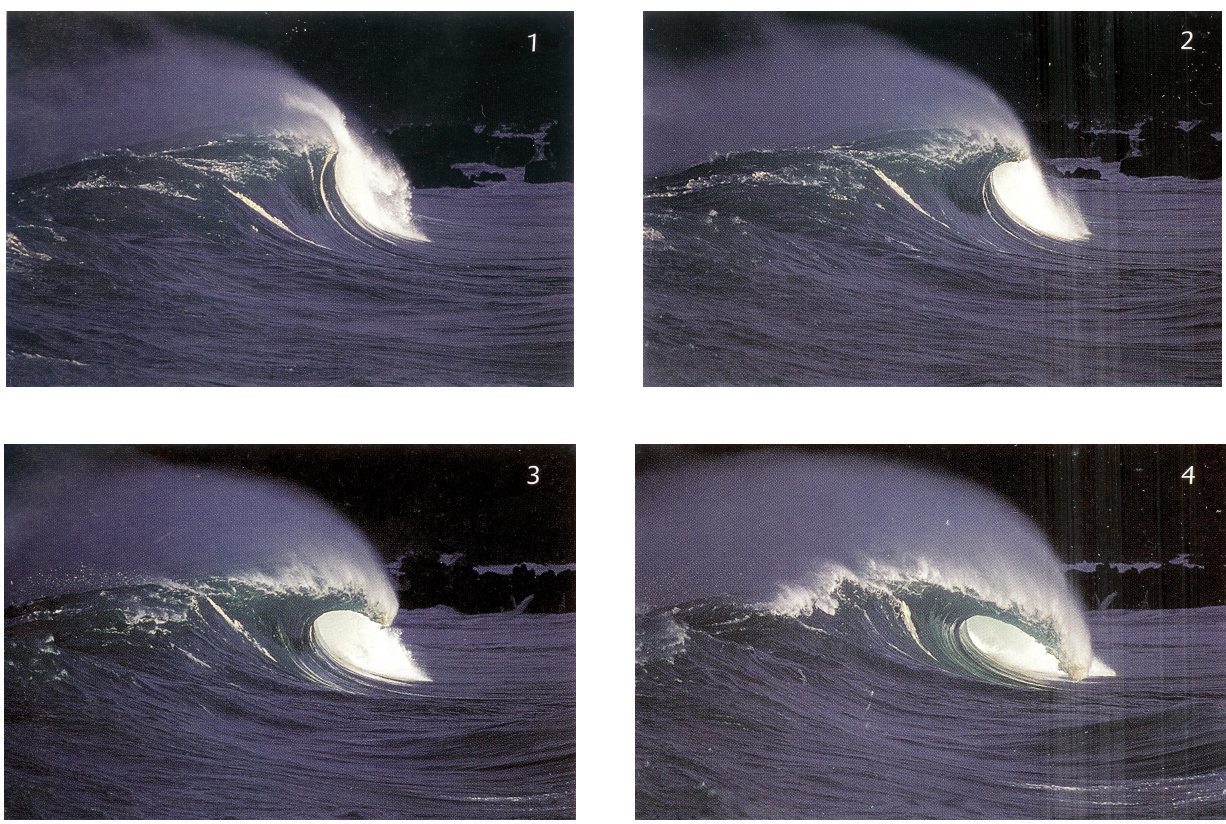

Figure 1.12: Sequence from left to right, top to bottom of a wave cresting and breaking [5].

factors make it difficult to predict the consistency let alone what the surface texture of the wave will be, and this is only one part of simulating an ocean environment.

The relative velocity vector of a fin with respect to the breaking wave is also difficult to measure. Not only is the face of the wave moving towards the beach, but there is water being pulled up the face as well. Most often these two vectors will be non-parallel, and the surfer will be moving and maneuvering independently of how the wave is moving. This makes it difficult to characterize the magnitude and direction of the fin's freestream velocity.

The final factor for consideration is the interaction of the surfboard bottom with the ocean surface. A surfboard will rarely be submerged evenly at the surface of the water. This is in in part due to the variety of bottom shapes (see Figure 1.13), and also to the uneven surface of the ocean. If there are wind gusts, the shear interactions along the ocean surface will create a choppy texture.

The combination of these conditions made modeling a realistic environment out 
SURFBOARD BOTTOM CONTOURS

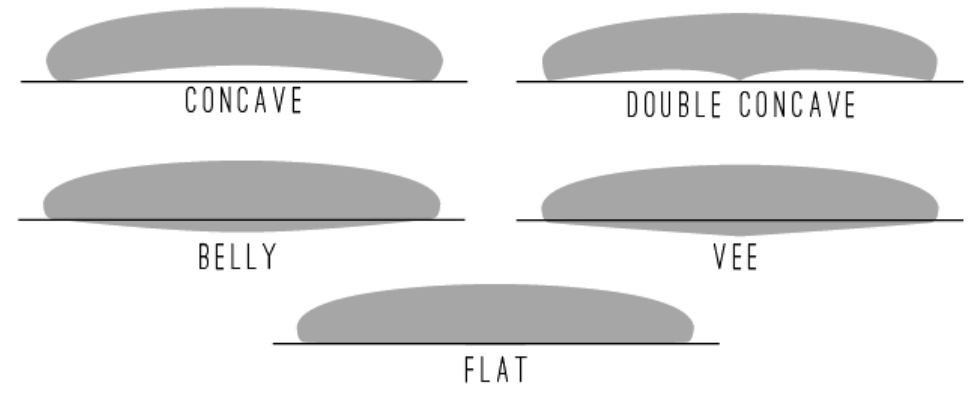

Figure 1.13: Cross sectional view of different surfboard bottom contours [6].

of the scope of this research. Instead, a simpler approach was taken: a surfer riding straight down a wave using a flat surfboard bottom. This removes the problem of characterizing the relative velocity, as the wave velocity will be assumed to be parallel with the surfboard velocity, and the surfboard bottom will be continuous with the top of the ocean, eliminating the interaction of air and salt water.

\subsubsection{Reynolds Number and Boundary Layers}

Using experimental data, basic properties of the wave can be characterized. A study was completed by Farley, Harris, and Kilding [25] during a surf competition to measure the physiological demands of competitive surfing. Twelve surfers were equipped with a heart rate monitor and an on-board global position system (GPS) unit that tracked the surfer's position in the water. The author's of the paper were interested in the athletic performance, but the GPS data was valuable for characterizing the average surfed wave.

After two events, the mean time for a surfed wave was 14.9 seconds. Meaning, the surfer only had close to 15 seconds to perform. In that time, the average wave riding speed was between 3.36 and $5.55 \mathrm{~m} \mathrm{~s}^{-1}$. This speed can be used to find the Reynolds number of the surfboard and fin. The Reynolds number describes the inertial to 
viscous ratio of a flow field with respect to a reference length. A low Reynolds number corresponds to a highly viscous fluid or slower velocity and vise versa for a high Reynolds number. Equation 1.2 shows the calculation of Reynolds number, Re, where $\rho$ is the density, $U_{\infty}$ is the freestream velocity, $l$ is a reference length, and $\mu$ is dynamic viscosity.

$$
R e=\frac{\rho U_{\infty} l}{\mu}
$$

Reynolds number is also used to describe whether or not the boundary layer is laminar or turbulent. The boundary layer is the region close to the surface where friction forces act on an object moving through a fluid. It stems from the no-slip principle, which states that at the surface of a moving object the mean velocity of the fluid is zero. The boundary layer is the region that captures the zero velocity at the surface to the point where the velocity has reached $99 \%$ of the freestream velocity. In relationship to the scale of the body the boundary layer is growing on, the boundary layer thickness, $\delta$, is proportionally much smaller [7].

Laminar flow is smooth and constant, and turbulent flow is chaotic and noisy. Whether or not the flow is laminar or turbulent is important for the amount of friction drag, or parasitic drag, an object experiences. Turbulent flow will have more momentum or velocity closer to the surface, generating more parasitic drag [7], and will also have a larger total boundary layer height.

At Reynolds numbers less than $3.5 \times 10^{5}$, more often than not the fluid will be laminar, and at Reynolds numbers larger than $1.0 \times 10^{6}$, generally the flow will be turbulent [7]. In between these two numbers, there is a transitional region shown in Figure 1.14. Depending on the environment, the point at which laminar flow transitions to turbulent varies. Flow characteristics that can influence this transition point are: the surface roughness, amount of turbulence in the freestream, type of 
pressure gradient, or thermal energy; which is why there is not always consensus to where laminar Reynolds numbers end and turbulent begins.

The Reynolds number for both the surfboard and fin can be found using conditions presented in the above study. From the average velocity range, $U_{\infty}=5 \mathrm{~ms}^{-1}$ was used to better represent a velocity closer to the faster speed regime. The density of salt water is $\rho=1026 \mathrm{~kg} \mathrm{~m}^{-3}$, and the dynamic viscosity is $\mu=1.23 \times 10^{-3} \mathrm{~N} \mathrm{~s} \mathrm{~m}^{-2}$ when the water is $15^{\circ} \mathrm{C}[26]$.

Shortboards, surfboards under $2.13 \mathrm{~m}(7 \mathrm{ft})$, were the only boards used in this competition. If surfboards ranging from $1.83 \mathrm{~m}(6 \mathrm{ft})$ to 2.13 meters are used, the Reynolds number for the bottom of shortboards span from $7.63 \times 10^{6}$ to $8.88 \times 10^{6}$, well into the turbulent regime. Fins, however, have a much smaller reference length. It is often better to use the MHC, but for the fins measured in Table A.1, the root chord was the only chord length measured, so this was used as the reference length. The smallest root chord measured was $106.0 \mathrm{~mm}$, and the biggest was $117.2 \mathrm{~mm}$, which equates to Reynolds numbers of $4.42 \times 10^{5}$ and $4.89 \times 10^{5}$ respectively.

These values sit within the transitional region for Reynolds number, but, because the bottom surface of the surfboard will likely have a turbulent boundary layer, the

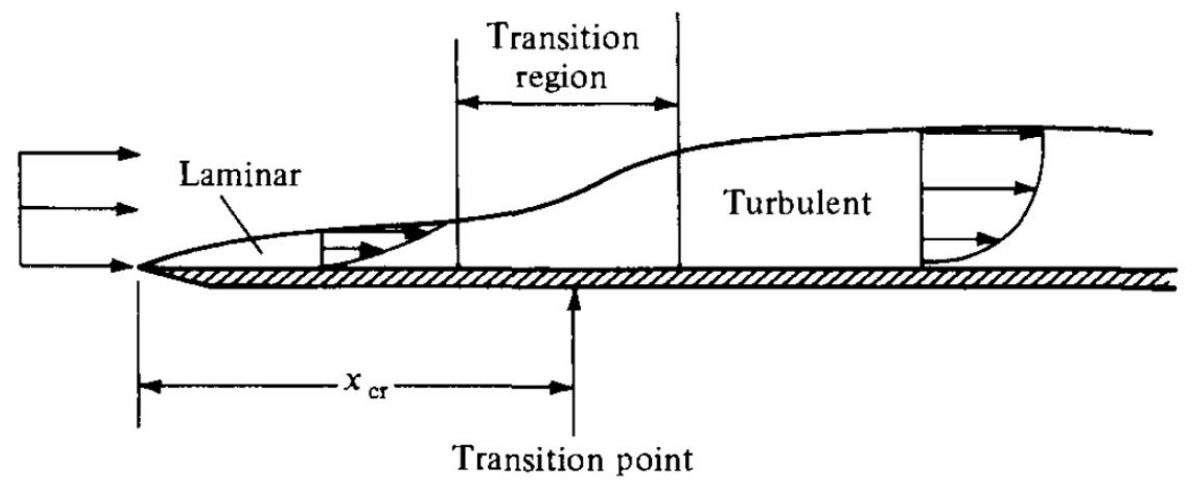

Figure 1.14: Illustration of boundary layer growth and transition. The image depicts boundary layer growth over a flat plate, and the solid line estimates the boundary layer height [7]. 
interaction of the air and ocean surface, and turbulent energy in the water, it is safe to assume that the flow field around the fin will be turbulent.

\subsection{Basic Fin Hydrodynamics}

Surfboard fin hydrodynamics are similar to classical aerodynamics. Some clarifications and definitions are made below.

\subsubsection{Body Forces}

A fin will generate body forces using the same principles of an airfoil and a wing. Surfboard fins have a hydrofoil cross section that create a difference in pressure along the top and bottom surface of the fin. When integrated, the difference in pressure results in body forces normal and parallel to the fin. These two forces on a wing are called lift and drag, and the single point where these two forces can be applied is the center of pressure, $c p$, of the fin.

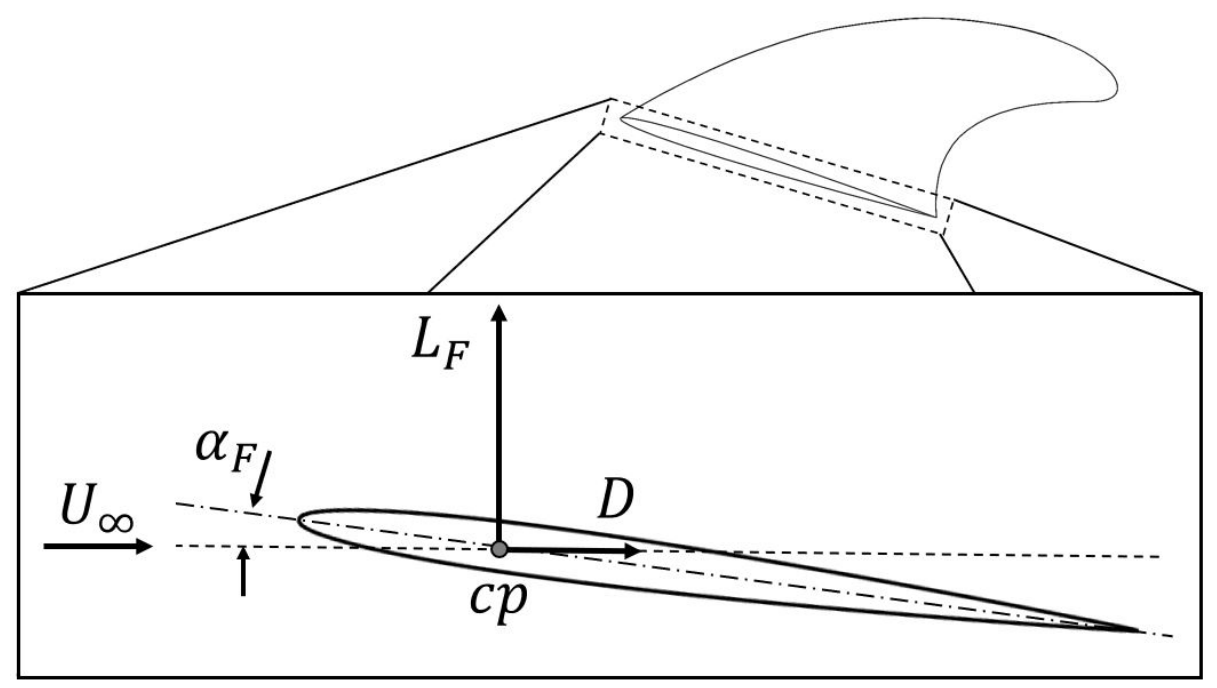

Figure 1.15: Horizontal cross section of root chord on fin, showing a sample hydrofoil, and a diagram of body forces with respect to a given freestream velocity vector.

The force normal to the fin is better described as a side force since it is not lifting 
the surfboard or fin but used to generate a turning force. Typically, the side force on the tail fin of an airplane is called the fin lift, $L_{F}$, so a similar notation will be used for the surfboard fin [27].

The angle at which the centerline of the hydrofoil sits at with respect to the freestream velocity is the incidence angle of the fin. This is more commonly called the angle of attack for an airplane wing, and for the fin it will be denoted as $\alpha_{F}$. Both the fin lift and drag can be non-dimensionalized for a given projected area, $S$, using Equation 1.3 and Equation 1.4.

$$
\begin{aligned}
C_{L_{F}} & =\frac{L_{F}}{\frac{1}{2} \rho U_{\infty}^{2} S} \\
C_{D} & =\frac{D}{\frac{1}{2} \rho U_{\infty}^{2} S}
\end{aligned}
$$

\subsubsection{Moments}

The fins turn the surfboard by generating a moment, $M$, between the center of gravity, $c g$, and the hydrodynamic center, $h c$. The center of gravity is the point where the weight force is applied for the surfer and surfboard system. A surfboard is typically made out of a light foam, so the surfer will proportionally outweigh the surfboard and fin, dominating the position of the $c g$. The distance between the $c g$ and $h c$ is the fin arm, $l_{F}$.

The moment can be calculated by multiplying the side force with the fin arm.

$$
M=L_{F} \cdot l_{F}
$$

The moment can also be non-dimensionalized, similar to the body force coefficients, using Equation 1.6, where $l$ is a characteristic length. For this system, the $M H C$ of the fin will be the characteristic length.

$$
C_{M}=\frac{M}{\frac{1}{2} \rho U_{\infty}^{2} S l}
$$




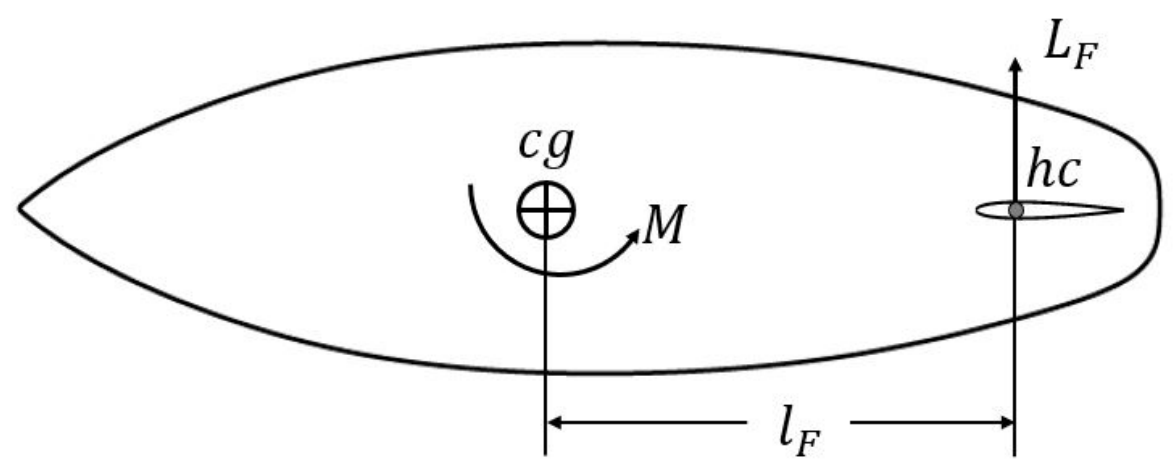

Figure 1.16: Diagram of the moment generated from the fin lift force.

\subsubsection{Vortex Shedding}

A vortex can form several ways. There are wing-bound vortices caused from the circulation around a wing, or wing tip vortices generated from the high pressure underneath a wing curling to the low pressure above the wing at the tip [8].

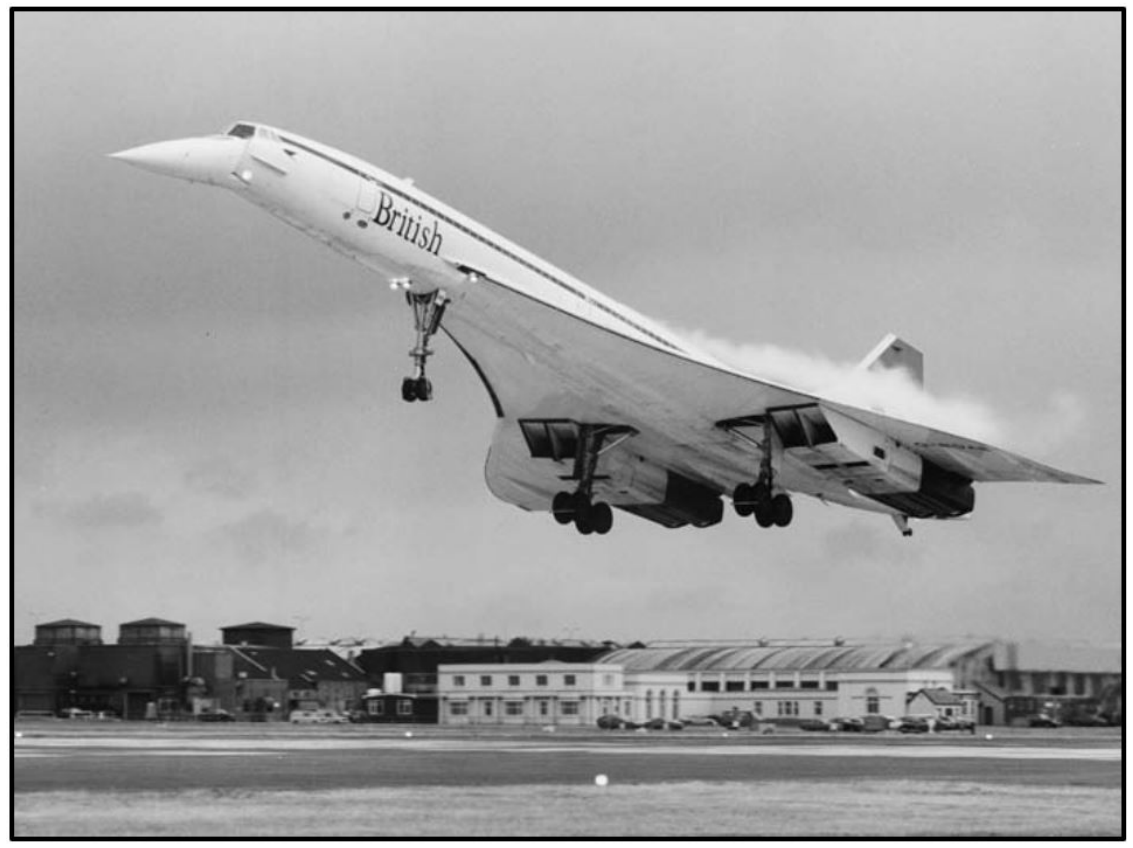

Figure 1.17: The strong conical vortex can be seen in the right conditions on the Concorde as condensed water vapor [8].

There is another type of votex used to produce lift on low aspect ratio wings. 
This type of lift is called conical vortex lift, and it is created through swept wings and separated airflow. Separated flow occurs when the fluid can no longer follow the curvature of the object it's traveling around. The boundary layer experiences an adverse pressure gradient too great, and the flow reverses at the portion of the boundary layer closest to the wall. Instead of wrapping with the object, it separates and creates a turbulent wake of low pressure and recirculating flow.

Separation causes large increases in drag, loss of lift, and instability, but separation can also be used advantageously in certain scenarios. "...if the wings are swept back at a sharp angle, the separated flow will roll up into a pair of stable cone-shaped vortices..." - Barnard and Philpott discussing the formation of the conical vortex on low aspect ratio wings [8]. An example of this concept demonstrated was on the Concorde, seen in Figure 1.17.

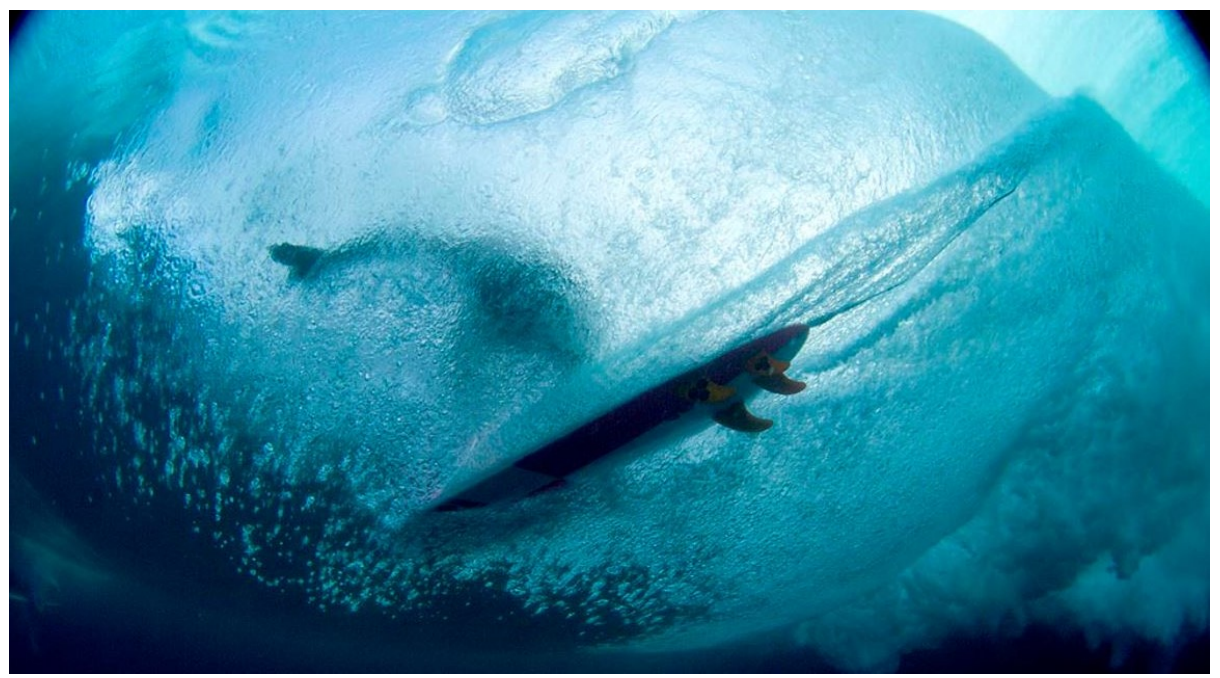

Figure 1.18: Underwater image of a surfboard with a thruster setup passing by. ${ }^{3}$

The speed at the center of the vortex is high compared to freestream, so the upper surface of the wing will generate lift. Strong conical vortices are most prevalent on low aspect ratio wings, triangular delta wings, or low aspect ratio surfaces with sharp

\footnotetext{
${ }^{3}$ https://surfsimply.com/surf-coaching/how-a-surfboard-works/
} 
leading edges. A surfboard fin fits this description, and although it does not have a sharp leading edge, it is thin enough to make it a candidate geometry.

To date, this has not yet been proven experimentally, but a vortex trail is often seen behind fins. For example, Figure 1.18 shows vortex trails coming off a surfboard and fins going across a wave. For these reasons, vortex shedding and the vorticity in the flow field around a fin was one focus of this research.

\subsection{Previous Work}

For the popularity of surfing, there have been surprisingly few published experiments on surfboard fins. One of these was performed at Cal Poly San Luis Obispo in their low speed wind tunnel [9]. This was a series of wind tunnel tests on a thruster configuration using only the FCS K2.1 fin geometry.

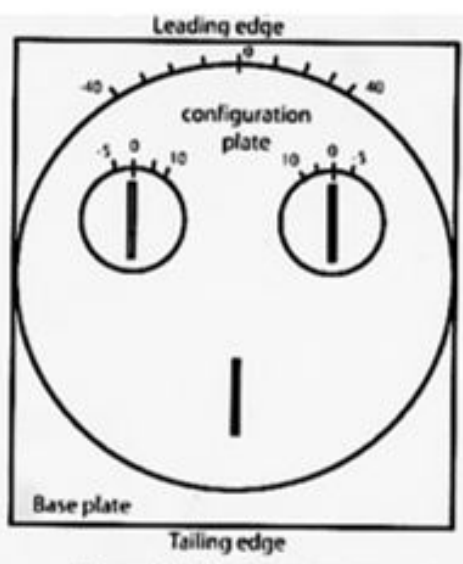

Figure 4 - Equipment setup

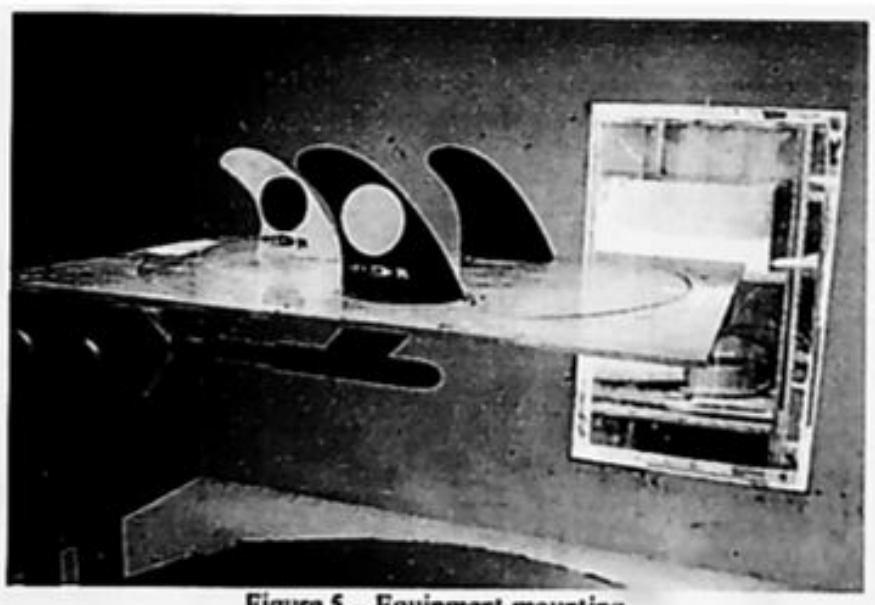

Figure 5 - Equipment mounting

Figure 1.19: (Left) Illustration of wind tunnel experiment setup. (Right) Picture of wind tunnel model [9].

The wind tunnel tests were run at Reynolds numbers ranging from $8.3 \times 10^{5}$ and $1.5 \times 10^{6}$, and the toe angle of the side fins were varied in increments of $5^{\circ}$. In addition, the incidence angle of the entire configuration was changed in increments of $5^{\circ}$ (see Figure 1.19 for the wind tunnel setup). The experiments measured the body 
forces on the fin: fin lift and drag.

The goal set by the authors was to demonstrate the feasibility of aerospace testing on surfboard fins, which they accomplished successfully. They found that each set of configurations stalled close to $20^{\circ}$ total angle of attack, and the fin configuration with $0^{\circ}$ toe angle performed the best, with a minimum drag force of $0.38 \mathrm{~N}$ and a maximum side force of 3.20 N. However, Tong and Millard acknowledge flaws in their testing and admitted that their results might not be completely accurate. Their testing apparatus moved during experimentation and there were problems in manufacturing, making their results less reliable.

A visual water tunnel experiment was completed by Brandner and Walker [4]. Their study focused on a single keel fin geometry, at Reynolds numbers ranging from $2.0 \times 10^{5}$ to $1.0 \times 10^{6}$ and incidence angles from $-2^{\circ}$ to $26^{\circ}$. The fin was tested at the Tom Fink Cavitation Tunnel, a closed recirculating variable pressure water tunnel. The fin had a root chord length of $0.1 \mathrm{~m}$ and a span of $0.12 \mathrm{~m}$. The experiments measured the fin lift, drag, and moment coefficients, along with taking high resolution flow visualization images at $R e=4.0 \times 10^{5}$ (Figure 1.20$)$.

The images were taken using two different techniques: off and on body. The off body techniques visualized water flow around the fin by injecting air bubbles with a reverse pitot-tube, and the on body technique used a mixture of titanium dioxide powder and silicone oil to generate oil streaks on the surface of the fin. The experiment showed a vortex coming off the tip of the fin from the air bubbles, and the on body flow highlighted a tendency for span-wise flow along with a clear transition from laminar to turbulent. At the $\alpha=20^{\circ}$ case, it also appears that there was large recirculation region near the leading edge of the fin and out towards the fin tip.

From the body force measurements taken, there was no discernible difference between the different Reynolds numbers except at $R e=2.0 \times 10^{5}$, where there were 

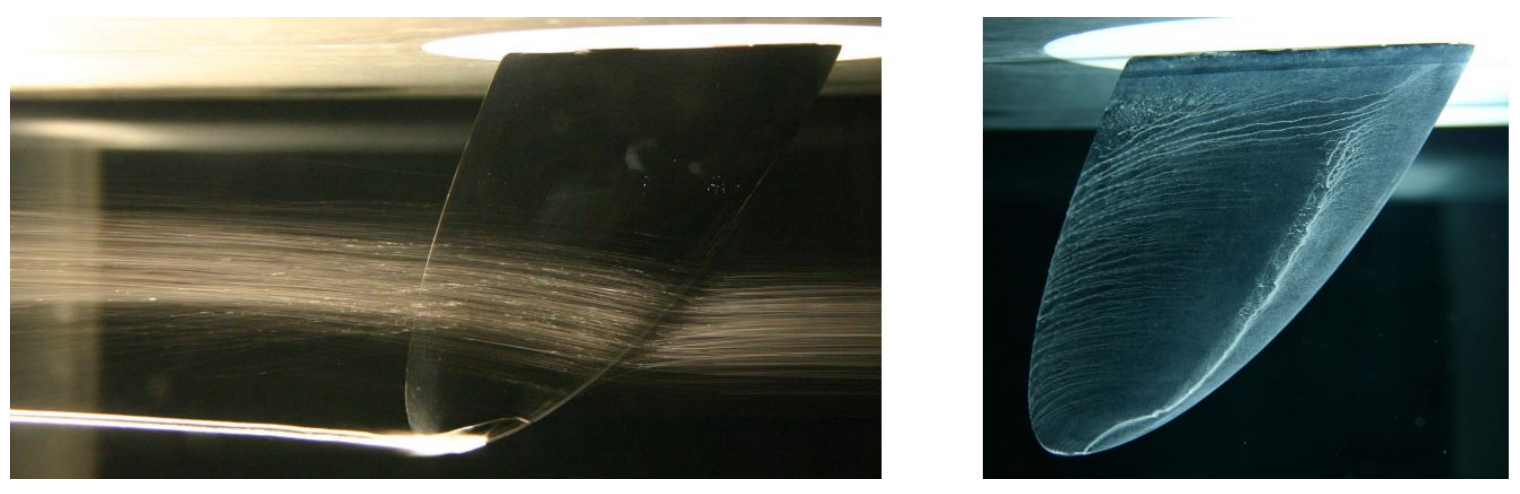

(a) (Left) On body and (Right) off body flow visualization at $\alpha=12^{\circ}$.
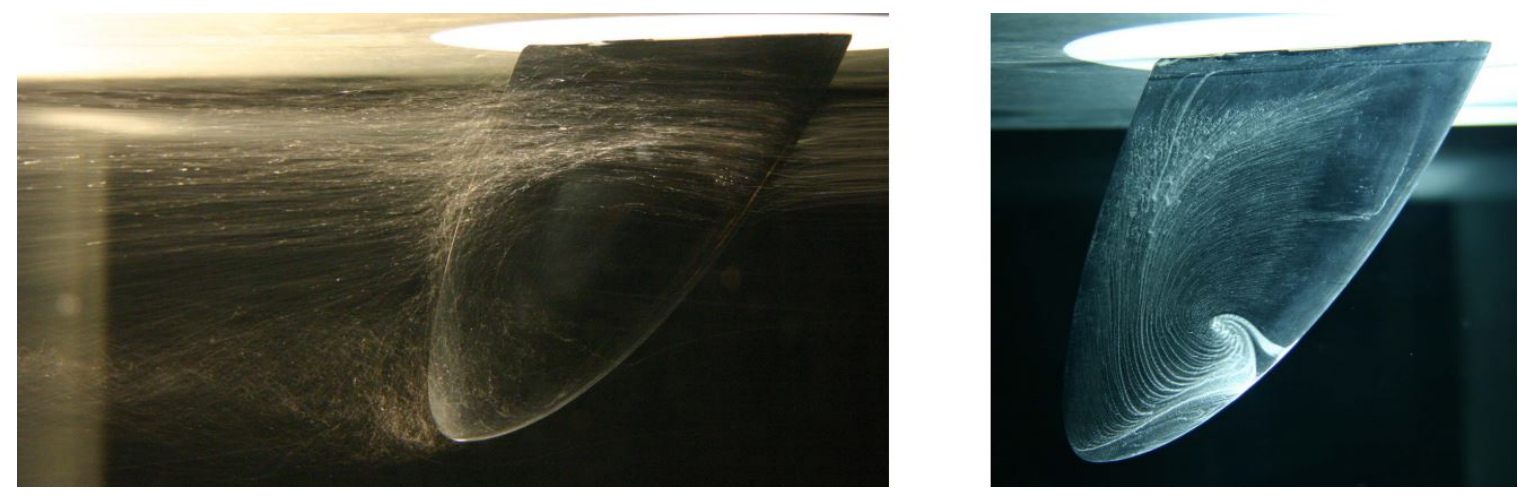

(b) (Left) On body and (Right) off body flow visualization at $\alpha=20^{\circ}$.

Figure 1.20: Flow visualization images of the tested keel fin at $R e=$ $4.0 \times 10^{5}$. The left images are off the body of the fin using air bubbles and the right images are on the body using oil [4].

noticeable instabilities. Their paper quoted these instabilities coming from laminar separation bubbles. There was no flow visualization for those cases, so it was hard to say whether or not this was true.

A computational fluid dynamic (CFD) and water tunnel study was completed on bio-inspred fin shapes by MacNeill [10]. Nine different marine animals' dorsal fins ranging from a Blue Shark to a Blue Marlin had their dorsal fins used as templates for surfboard fin shapes created in CAD software. The CAD was then used to print water tunnel models and was then imported into CFD software. The two sets of testing were validated against each other.

MacNeil was attempting to characterize how the different shapes affected the 
lateral stability and body forces of surfboard fins and chose to use nature as her inspiration. The CFD was run using Reynolds Averaged Navier-Stokes (RANS) based models and a $k-\epsilon$ turbulence model. A tetrahedral mesh was used with cell counts averaging between $3.9 \times 10^{4}$ to $8.0 \times 10^{4}$.

The water tunnel tests were completed at Michigan Technological University and used a spring gauge to measure forces. The models were printed using Polylactic Acid (PLA) plastic and tested at $R e=5.57 \times 10^{4}$, referencing a root chord length of 112 $\mathrm{mm}$.

From both series of tests, MacNeill found that the Short-finned Pilot Whale had the best lift-to-drag ratio in both the CFD simulations and water tunnel testing at 4.45 and 4.77 respectively. However, there were some problems with the methodology that make these results questionable. For instance, the CFD simulations were not preceded by any mesh or domain independence studies, making the relatively low cell count suspicious. The author also notes that the instrumentation used to measure the water tunnel forces was very likely to be inconsistent. The spring gauges were not electrical, all recorded force values were taken by eye sight, and the increments of measurement were in tenths of a newton. This makes precise accuracy much harder to obtain.

Another issue was that every fin geometry was scaled unproportionally. As can be seen in Figure 1.21, each fin was stretched to reach a uniform span length. By doing so, the wing loading of the fin changes from the original geometry and makes it difficult to correlate the two.

While the breadth of experiments and testing completed by MacNeill was comprehensive, it may not be a reliable source for hydrodynamic performance. There was not much correlation from the hydrodynamic results to the geometry change of the fin either. 

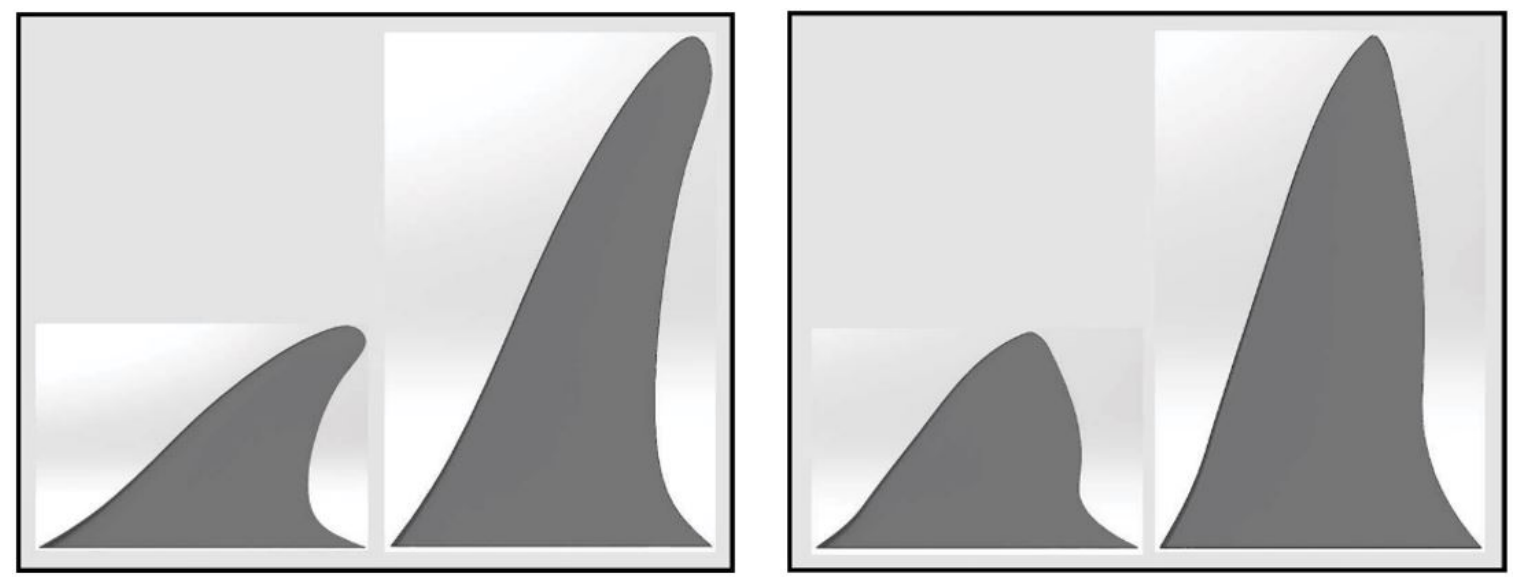

Figure 1.21: (Left) Fin Whale and (Right) Blue Shark fin's rescaled to a larger span [10].

These experiments cover a majority of published work on surfboard fins, and no conclusive statements can be made from them. Other research not mentioned focus on multi-fin configurations, which does not aid in the analysis of a singular fin shape. The result is a lack of experiments on a single, isolated fin. 
Chapter 2

\section{OBJECTIVES}

\subsection{Problem Statement and Goal}

No correlation has been made to the size and shape of a surfboard fin and its performance. While there are subjective grading criteria, not much headway has been made in quantifying the fin itself. This research's goal was to isolate geometric parameters on a surfboard fin to understand how each parameter influences the flow field. The two parameters chosen to vary are the sweep and taper of the fin.

The primary method of generating results was done through computational fluid dynamics (CFD) simulations. Once results were generated, the goal was to correlate how differences in the parameters effect the performance of the fin and the performance of a surfer using the fin.

\subsection{Methodology of this Study}

This study uses CFD to analyze a single, symmetrical fin without the flow field influence of a multi-fin configuration. This is done to gain a more precise understanding that can then be applied to future design considerations. The FCS II FT [11] was used as a baseline shape because of it's popularity and similarity to other fin designs. This shape had it's sweep or taper changed using discrete point methods, and the newly generated planform shape was used to create a three-dimensional CAD model.

The CAD model was then imported into CFD software to simulate a simplified salt water environment in a rectangular fluid domain. The underside of the surfboard was modeled as a flat, no-slip surface, and the fin was placed proportionally on this 
surface. The flow field was assumed to be steady, and the Reynolds Averaged NavierStokes (RANS) equations were used with a $k-\epsilon$ realizable turbulence model. Greater detail of the numerical methodology and simulation are described in later sections.

A total of 15 different geometries were tested at a surf fin Reynolds number of $3.51 \times 10^{5}$. This can be considered a transitional regime of Reynolds numbers, but as reasons described in Section 1.41, the flow field was assumed to be turbulent. Each fin geometry was swept through incidence angles of $0^{\circ}$ to $20^{\circ}$, and fin lift, drag, and moment coefficients were reported at each angle. These values along with images taken of scalar scenes were used to compare the results for the different geometry. 
Chapter 3

GEOMETRY

\subsection{Hydrofoil Selection}

The hydrofoil of a surfboard fin is the two-dimensional cross section, analogous to the airfoil on a tail fin. While both hydrofoils and airfoils are critical to the performance of a fin, the goal for this study was not to optimize the hydrofoil used for surfing. Because of this, the hydrofoil selection was not considered significant to the overall study. It did remain constant for each shape created so that it did not influence any changes made to the planform shape.

Choosing a hydrofoil involved measuring 25 manufactured surfboard fins for their root chord length and maximum thickness with a Neiko 01407A Digital Caliper. The digital caliper had a resolution of $0.01 \mathrm{~mm}$, and the results of these measurements are tabulated in Table A.1 in the appendix.

The maximum thickness is the point on the hydrofoil with the thickest cross section, and determining this location by hand and eye sight was not an exact process. No imaging or machine tools were used to gather where the point of maximum thickness was located because the goal was to estimate on average what the thickness to chord ratio, $t / c$, was. From the 25 fins measured, 18 were side fins and 7 were center fins on a thruster fin configuration, with the exception of one five fin configuration (this configuration used the same thruster fins but added an extra fin on each side).

The average $t / c$ was $6.35 \pm 0.25 \%$ and $6.59 \pm 0.25 \%$ for the side and center fins respectively. When rounded to the nearest whole integer, each surfboard fin had close to a $6.00 \% t / c$. The $t / c$ percentage was rounded to simplify the hydrofoil selection. 
Typically the side fins on a thruster configuration will use an asymmetric hydrofoil, and the center fin will use a symmetric hydrofoil. This study isolated the symmetric center fin of a thruster configuration, so the choice was to use a symmetric hydrofoil with $6.00 \% t / c$. Using the NACA four digit airfoils, this meant that a logical selection for the hydrofoil was the NACA 0006. The NACA four digit airfoils were created using a set equations that describe the mean camber line and thickness; the first two digits represent the camber of the hydrofoil, and the second two are equal to the $t / c$. Therefore, the NACA 0006 has no camber, is symmetrical and has a maximum $t / c$ of $6.00 \%$, which fits the profile needed for the surfboard fins.

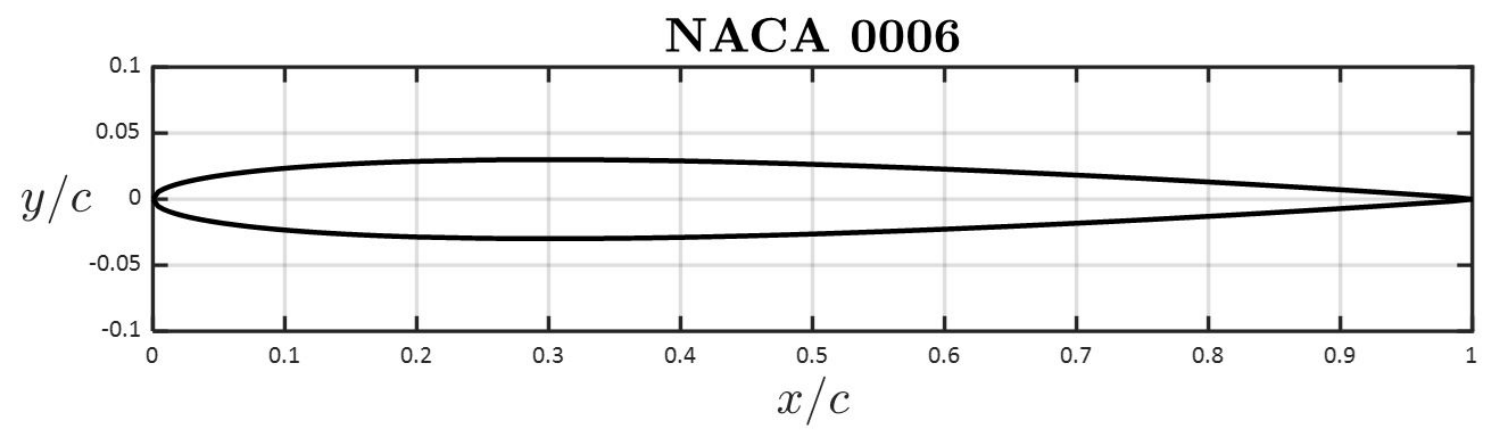

Figure 3.1: NACA 0006 Airfoil.

\subsection{Fin Sweep Definition}

There were two variables used to change the geometry of the fin: the sweep and taper. The sweep angle, $\Lambda$, is the angle referenced from the root half-chord to the fin tip and is the shown in Figure 3.2a. As an aside, the half-chord was used as a reference instead of the quarter-chord because it was not influenced by the taper of the fin. The angle depicted in Figure 3.2a would only be true for a fin with constant sweep, which was not the case for this geometry. The sweep of the fin was variable with respect to the span of the fin. The angle shown is $\Lambda_{t i p}$, or the sweep of the fin with respect to the fin tip. 
This can be illustrated by following the centerline of the fin. The centerline references the half-chord location along the span of the fin. If the sweep was constant, the line from fin tip to the root half chord would overlap with the centerline, but as seen in Figure 3.2b, this wasn't true.

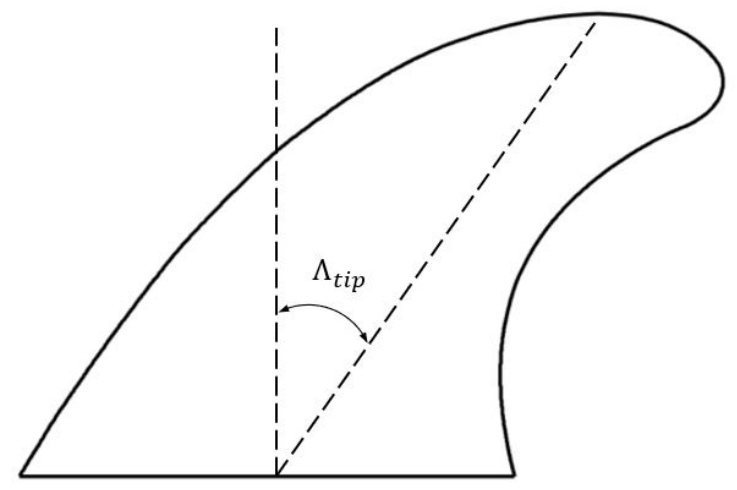

(a)

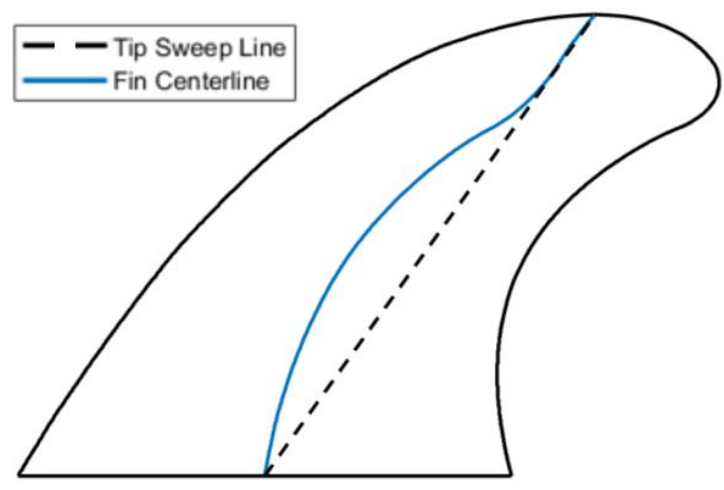

(b)

Figure 3.2: (a) Fin sweep defined from the root half-chord to the fin tip. (b) $\Lambda_{t i p}$ compared to the centerline of the fin.

The sweep was instead changing and a function of the span, $\Lambda(y)$. To match this description, the sweep for a given height, $y$, was measured from the root half-chord to the centerline at that location. This is shown in Figure 3.3 and will be used to describe the sweep distribution along the span.

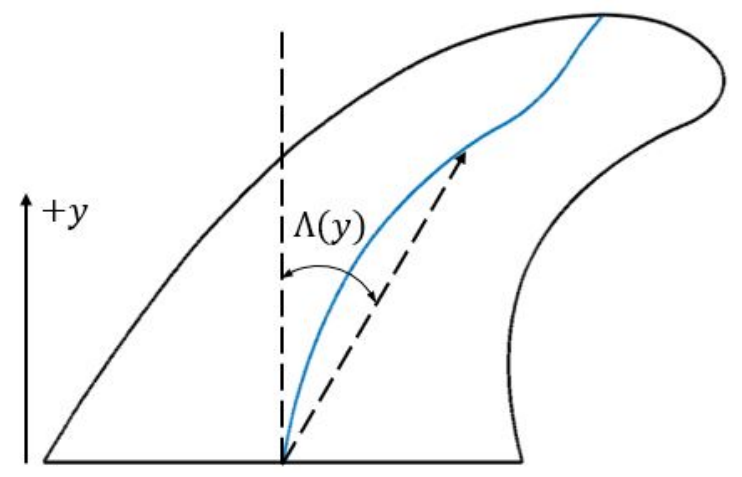

Figure 3.3: Sweep of the surfboard fin as a function of the span location. $\Lambda(y)$ is defined as the sweep at location $y$ from the root half chord to the centerline. 


\subsection{Fin Taper Definition}

The taper of a wing is the change in chord length with respect to the root chord. For constant, tapered wings, this relationship can be defined by Equation 3.1, where $c_{t}$ is the tip chord length. A taper ratio bigger than one corresponds to a tip chord larger than the root chord, and a taper ratio less than one is a tip chord that is smaller.

$$
\lambda=\frac{c_{t}}{c_{r}}
$$

However, this definition only applies to geometries that are trapezoidal, as can be seen in Figure 3.4a. Not only is a surfboard fin non-trapezoidal, but the tip of the fin also comes to a point. A point has a reference length of zero, therefore $\lambda=0$, and applying Equation 3.1 was less meaningful and not representative of the fin shape.

Similar to the sweep, the fin had variably changing taper. The taper ratio was a function of the span, $\lambda(y)$, and can be defined by Equation 3.2. Instead of a singular value, $\lambda$ varied with height. $c_{y}$ is the chord length at a given span location $y$, and $c_{r}$ is the root chord.

$$
\lambda(y)=\frac{c_{y}}{c_{r}}
$$

An illustration of this definition can be seen in Figure 3.4b. 


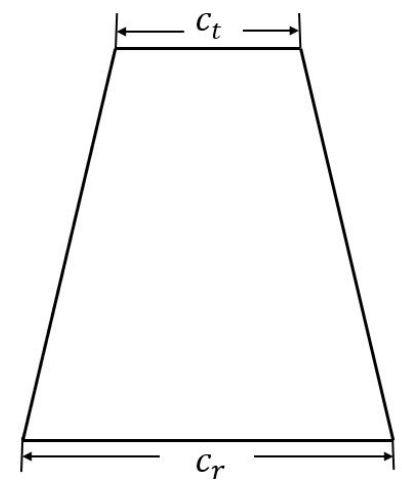

(a)

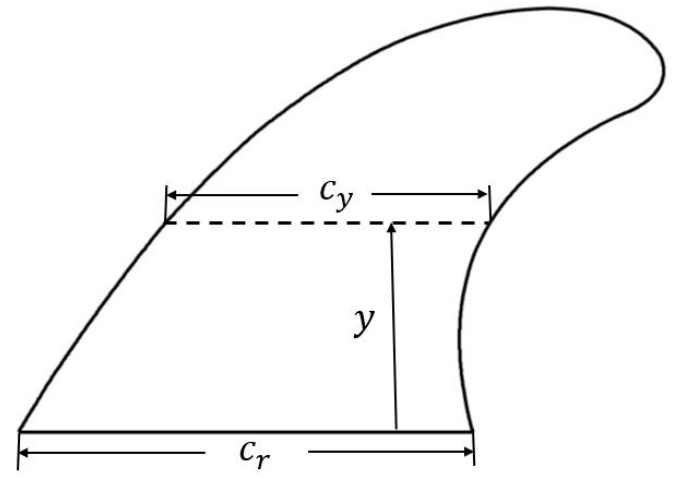

(b)

Figure 3.4: (a) Rectangular wing taper. (b) Taper measurements for a surfboard fin, where $y$ is measured from $c_{r}$. 
Chapter 4

THREE DIMENSIONAL MODELING

\subsection{CAD Methodology}

The CAD models were created using three steps. The first step was to use the image of a pre-existing fin geometry as a template for a baseline configuration. The image was imported and processed using Matlab software, and the edge of the fin was represented as discrete, non-dimensional points. These points were manipulated and shifted in order to create different shapes for surfboard fins and exported to a CAD software. The CAD software generated a 3D model of the fin that was exported for use in CFD.

\subsection{Constraints}

There were restrictions placed on what and how much could change for the geometry. The span and projected area of the fin remained constant unless otherwise noted. This was to ensure that the results were comparable for a given Reynolds number, and to isolate the other design variables. The two variables that were changed on the fin were the sweep and taper distribution. In order to isolate one of those variables, the other was left unaltered. For example, if the sweep of the fin was changed, the taper distribution remained the same. In doing so, the simulations showed the effects of changing a single variable.

The only limitation placed on the sweep distribution was that $\Lambda(y) \not \leq 0$. This meant the leading edge of any cross section did not go in front of the root chord leading edge and ensured no forward swept values. This was done to not only limit 
the scope of this research, but because a forward swept fin is not practical when surfing. A forward swept fin can accidentally catch seaweed or other debris as it travels forward. The ocean is different than flying in open air space. There are plenty of living and non-living objects in an aquatic environment that can become tangled on surfing equipment. While it is not formally documented, shedding debris is one reason why a surfer would want to have sweep with their fin.

The taper will have a similar restriction to the sweep in that $\lambda(y)>0$ except at the fin tip. There cannot be a chord length on the fin that collapses to a point before the tip.

\subsection{Baseline Shape}

The baseline shape was the starting, reference geometry. The FCS II Filipe Toledo Tri Fins (FCS II FT) are a fin set manufactured by FCS (Fin Control Systems) for a thruster configuration and were chosen for the baseline shape, seen in Figure 4.1 [11]

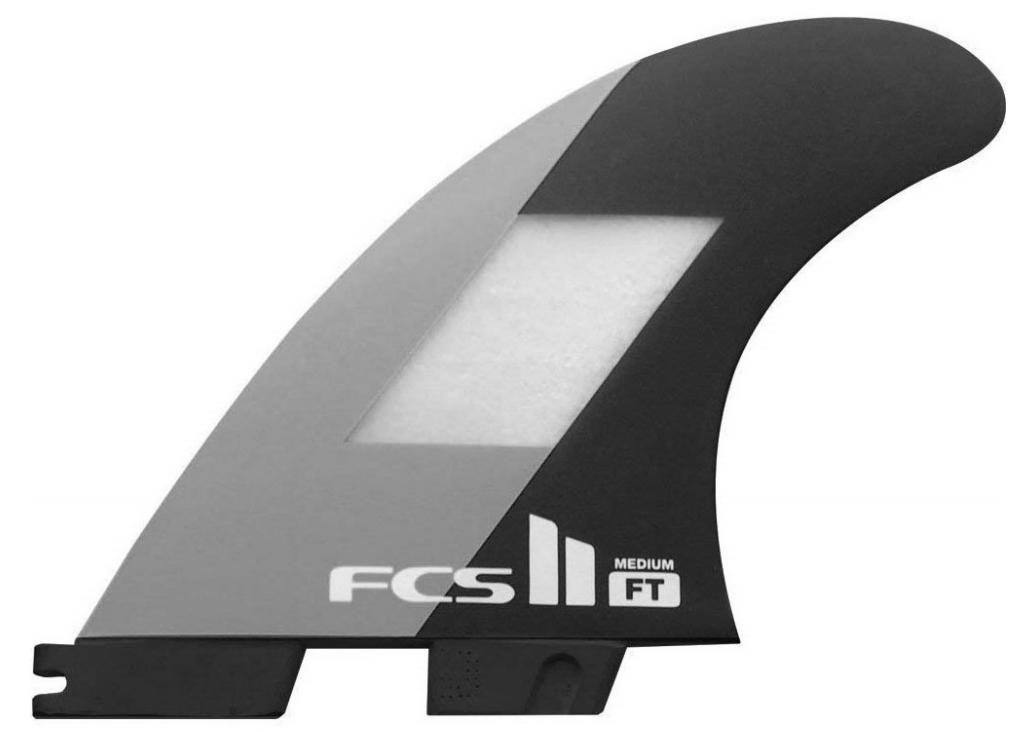

Figure 4.1: FCS II FT fin. The image shown is the center fin for the medium size fin set [11]. 
The choice for a baseline shape was not critical to the results of the research, as it is the starting point. As long as the rest of the changes refer back to this shape, the end results were meaningful. With that said, the FCS fins were not chosen arbitrarily; as of the current date, this particular fin is widely sold and commonly used, meaning results gained are applicable. It also has an aspect ratio close to one, which was convenient for calculations, and when compared to other fin shapes and dimensions provided by FCS, it was close to a median value [1].

Table 4.1: Baseline fin dimensions [1].

\begin{tabular}{cccccc}
\hline$c_{r}(\mathbf{m m})$ & $M H C(\mathbf{m m})$ & $b(\mathbf{m m})$ & $S\left(\mathbf{m m}^{2}\right)$ & $A R$ & $\Lambda_{\text {tip }}$ \\
\hline 115 & 85.7 & 118 & $1.01 \times 10^{4}$ & 1.37 & $35.2^{\circ}$ \\
\hline
\end{tabular}

One thing to note about the image in Figure 4.1 are the two protrusions at the bottom of the fin. These are the box tabs that insert into a fin box on a surfboard, and it's how the fin attaches to a surfboard. Moving forward, the fin tabs were ignored as they are flush with the surface of the surfboard and do not protrude into the free stream flow.

\subsubsection{Image Processing}

The image chosen was free of any perspective and perfectly orthogonal to the planform of the fin. Using Matlab, the image in Figure 4.1 was traced to generate a baseline shape. Matlab has built in image post-processing tools that can import and convert image file types into discrete coordinates. The process started by first using a file reading function that converted each pixel into a position with an associated red, green, and blue color strength value. Once the raw image data was imported, the next step was to find the outermost coordinates that defined the edge.

Depending on the background of an image, the easiest way to separate the border of the object from the background was too look for a transition from a given color to 
a different one in adjacent pixels, for example, red to black or green to white. The easiest transition to look for was from black to white, as black and white pixels will only have zero's or one's in the red, green, and blue color space. Because of this, the image of the fin was converted to all black pixels and the background to all white.

The last step before moving forward was removing the fin tabs and was done by cropping the bottom portion of the fin. Figure 4.2 shows the result of this process and was the image used to separate the border of the fin with the background. When Figure 4.2 was imported, a matrix of values representing the black and white pixel locations was generated and then restructured with a built-in Matlab function to create a two column matrix of $\mathrm{x}$ and $\mathrm{y}$ coordinates of edge values.

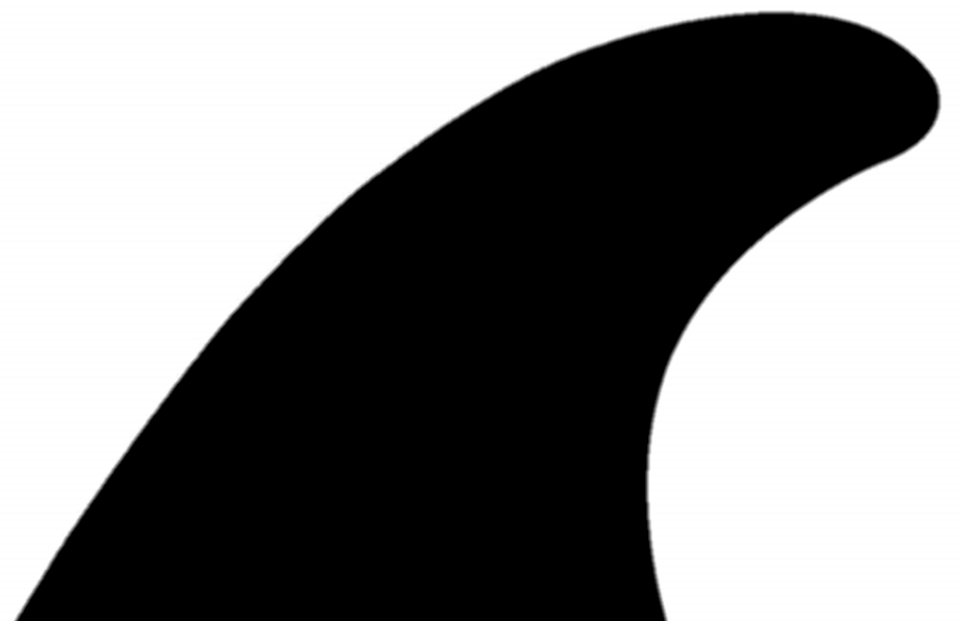

Figure 4.2: Edited image used to trace the edge of the baseline, planform shape.

The imported coordinates did not have either SI or Imperial units attached to them. As they were imported, each coordinate had an associated location based on the resolution of the image, see Figure 4.3a. This was addressed by first normalizing the coordinates with the length of the root chord in pixel coordinates. The root chord then had a length of one, and the rest of the edge values were scaled in a similar manner. The entire coordinate set was then translated to an origin of $(0,0)$.

At this stage, the edge values were scaled and ordered properly to represent the 
outline shape, but still did not have attached units. The decision was made to maintain a root chord unit length of one and to use meters as the units. While the original root chord length of the FCS II FT was $115 \mathrm{~mm}$, using that value makes future scaling more time consuming, and as long as the Reynolds numbers were matched properly, the decision to leave the root chord length as one meter did not influence results.

The edge points were then split into two halves: leading and trailing edge coordinates. This was done by finding the coordinate of the fin tip point, and splitting any points with a $x$ value smaller than $x_{t i p}$ as leading edge coordinates and vice versa for the trailing edge. The leading and trailing edge coordinates excluded the fin tip point and the line connecting the root chord.

An issue encountered here were coordinates with the same $y$ value on both the leading and trailing edge. Having two points at the same $y$ coordinate is the result of loading in the pixel distribution from the image. The fineness of a given curvature for a picture is only as good as the pixel resolution at the location. When displaying any kind of line that is not horizontal or vertical, the pixels will create a discrete stair step pattern to follow the line. This isn't always evident when looking from a zoomed out perspective, but when looked at closer, this problem can be seen in Figure 4.4.

This was problematic for defining the sweep and taper distribution, as there were two or more points too choose from at a given cross section, and it created ambiguity for which $x$ value to input for calculations. The extra points were removed by selecting either the center point of the set or the left center point for even numbered points and resulted in a single, unique $x$ coordinate for the leading and trailing edge for each $y$ height.

This kind of filtering process could have left slight discrepancies to the original geometry, as points were removed that defined the original shape. However, on average the distance between points in the $x$ direction was $0.0015 \mathrm{~m}$, so if two points were 


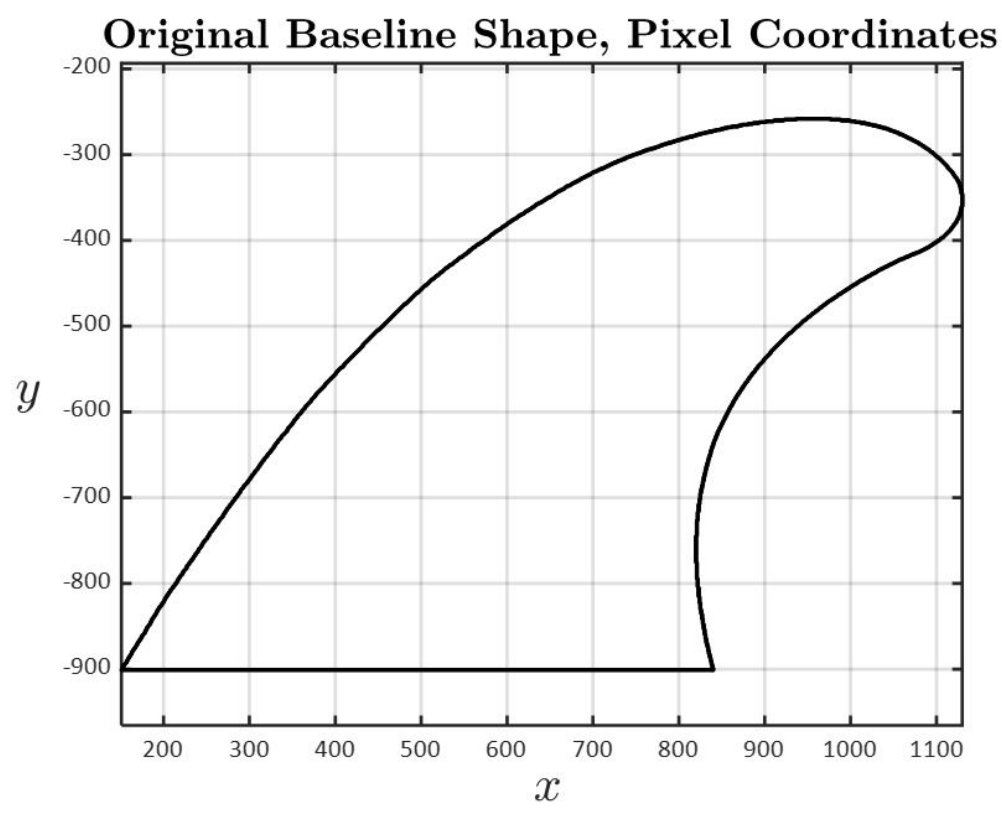

(a) Plotted edge coordinates of the image after Matlab processing. There are no units on the $\mathrm{x}$ or $\mathrm{y}$ axis as the locations are pixel coordinates from the image.

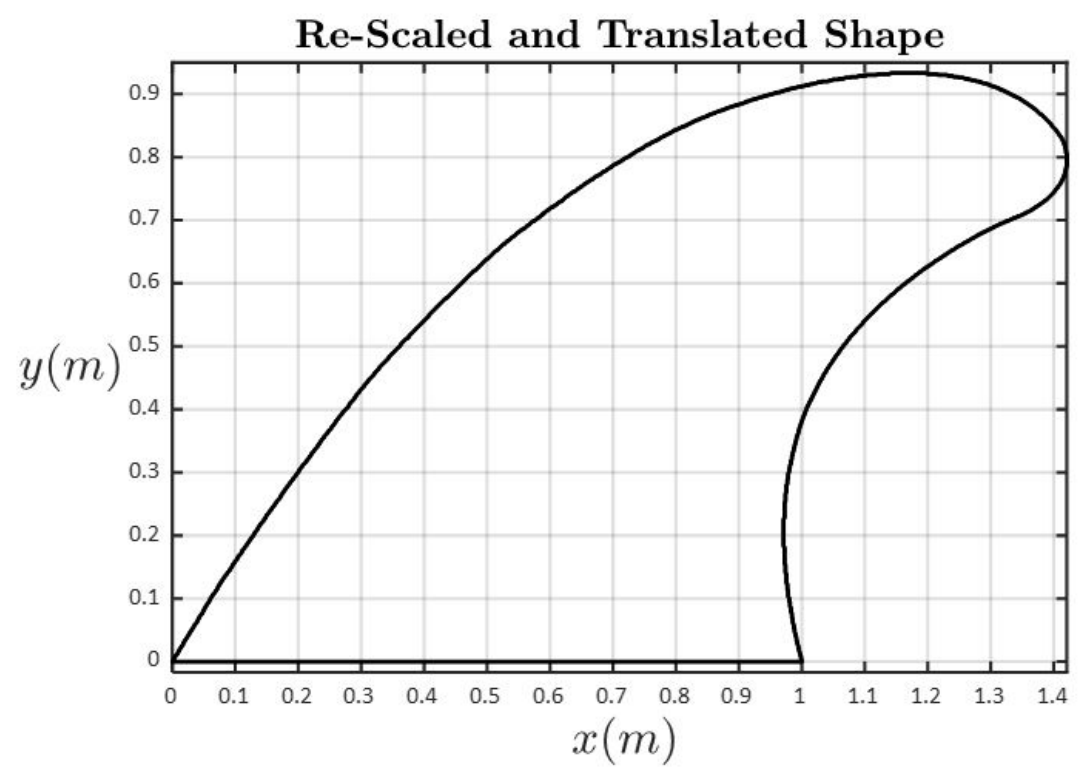

(b) Re-scaled and translated plot. The origin of the root chord leading edge is at a coordinate of $(0,0)$ and $c_{r}=1$. The aspect ratio and proportionality are kept constant Figure 4.3: Comparison of the edge coordinates after converting to units of meters and translating to an origin coordinate of $(0,0)$. 


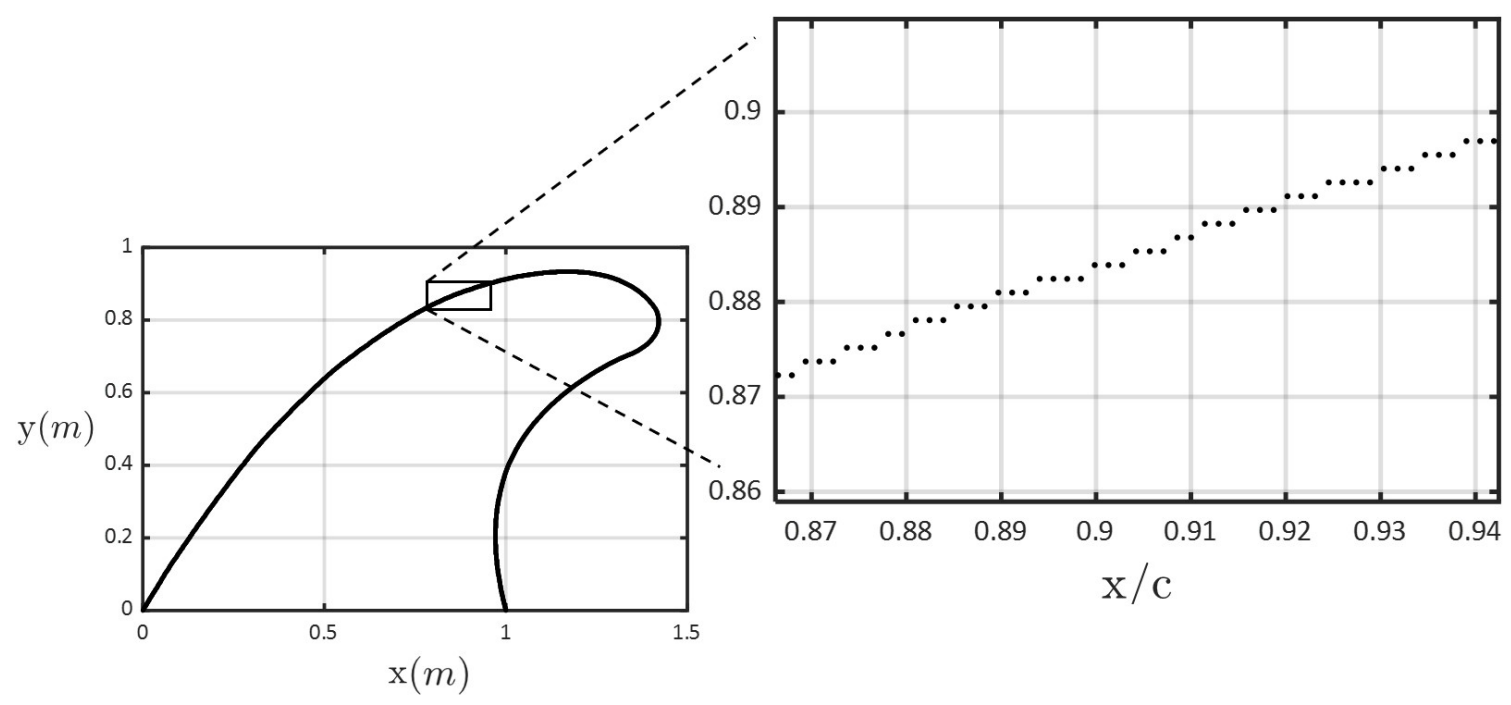

Figure 4.4: Zoomed in window of edge coordinates. Because there are more than one point at a given $y$ coordinate, the points create a stair step like shape.

removed, the outline of the shape would have to interpolate $0.003 \mathrm{~m}$. This is $0.3 \%$ of the root chord, and was assumed to be a small enough distance to interpolate with respect to the total size of the fin to not affect the overall planform shape.

This same filtering process did not need to be applied to $x$ values as it was impossible to have duplicate $y$ points for a given span location. This was because the cross sections were defined in the $y$ direction, not $x$. At this stage, the leading and trailing edge were successfully defined and the next step in the CAD creation could be taken. The final dimensions for the post processed fin are summarized in Table 4.2.

Table 4.2: Baseline fin dimensions after post processing.

\begin{tabular}{cccccc}
\hline$c_{r}(\mathbf{m})$ & $M H C(\mathbf{m})$ & $b(\mathbf{m})$ & $S\left(\mathbf{m}^{2}\right)$ & $A R$ & $\Lambda_{t i p}$ \\
\hline 1.00 & 0.745 & 0.933 & 0.695 & 1.37 & $35.2^{\circ}$ \\
\hline
\end{tabular}




\subsection{Two Dimensional Point Manipulation}

In Chapter 3, it was stated that both the taper and sweep of a surfboard fin vary non-linearly along the span of the fin. This presented a challenge for changing one parameters and not affecting the other. The sweep and taper each required a unique approach to change their respective distribution whilst leaving the other parameters constant.

\subsubsection{Calculating Fin Sweep}

To start changing the fin sweep, the original sweep distribution was calculated first. As stated previously, this was done by measuring the angle between the centerline at a given height to the half chord at the root and is illustrated in Figure 4.5.

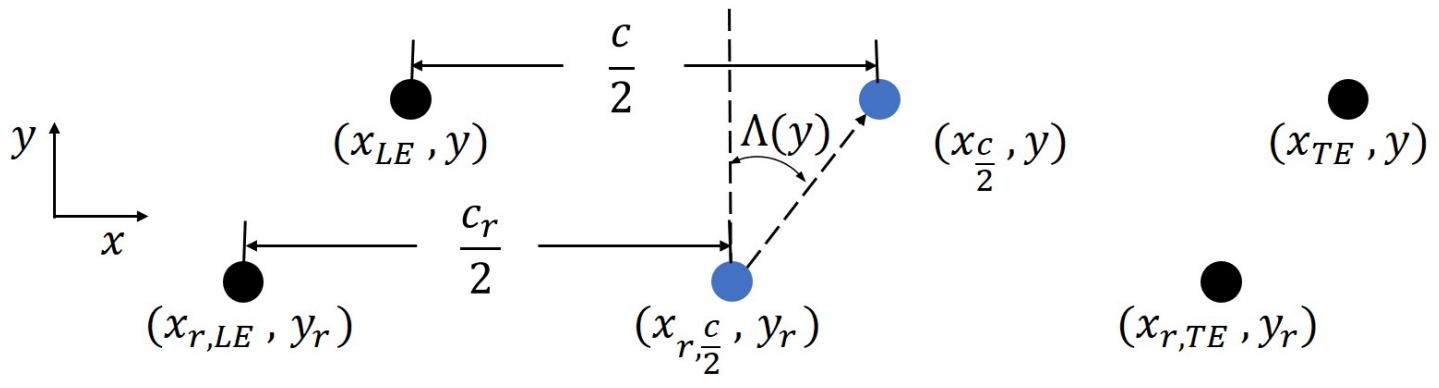

Figure 4.5: Discrete point illustration of how $\Lambda(y)$ is defined.

The subscript $r$ represents values taken at the root chord, and the second subscript seen in $x$ coordinates was for either the leading edge, $L E$, or the trailing edge, $T E$. Figure 4.5 shows the vertical line parallel with the y axis at $c_{r} / 2$ and the line connecting $c / 2$ and $c_{r} / 2$ used to define the sweep angle for each height. As different cross sections were referenced, the vertical reference line did not change, but the line connecting the two half chord locations changed depending on the offset of the centerline.

Calculating $\Lambda(y)$ was then a matter of trigonometry. The change in $x$ and $y(\Delta x$ 
and $\Delta y$ ) were used as reference lengths for this calculation, and to do so the location of the half chord point was found first.

$$
x_{c / 2}=\frac{x_{T E}-x_{L E}}{2}
$$

$\Delta x$ and $\Delta y$ were then calculated as follows:

$$
\begin{gathered}
\Delta x=x_{c / 2}-x_{r, c / 2} \\
\Delta y=y-y_{r}
\end{gathered}
$$

If the taper distribution is unaltered, the root chord remain constant, $x_{r, c / 2}=0.5$ and $y_{r}=0$. The equations then reduce to:

$$
\begin{gathered}
\Delta x=x_{c / 2}-0.5 \\
\Delta y=y
\end{gathered}
$$

With $\Delta x$ and $\Delta y$ calculated, $\Lambda(y)$ was then found with Equation 4.6.

$$
\Lambda(y)=\tan ^{-1}\left(\frac{\Delta x}{\Delta y}\right)
$$

Figure 4.6 shows the result of this calculation for the baseline fin, and as a result, the first portion of the graph contained noisy data. This stemmed from small, position changes close to the root chord. At cross sections and $y$ values close to the root chord, $\Delta y$ remains small, and any change in $\Delta x$ is proportionally much larger than cross sections farther away from the root chord. This resulted in more volatile values for $\Lambda(y)$ close to the root chord.

The data was still usable. Larger spikes in the distribution did not necessarily correlate to large shifts as $\Delta x$ and $\Delta y$ may both be small. The noisy information can be filtered using a built-in Matlab moving average filter, a variation of a low pass filter. This corrected most spikes in the distribution except for initial variations, seen in Figure 4.7. 


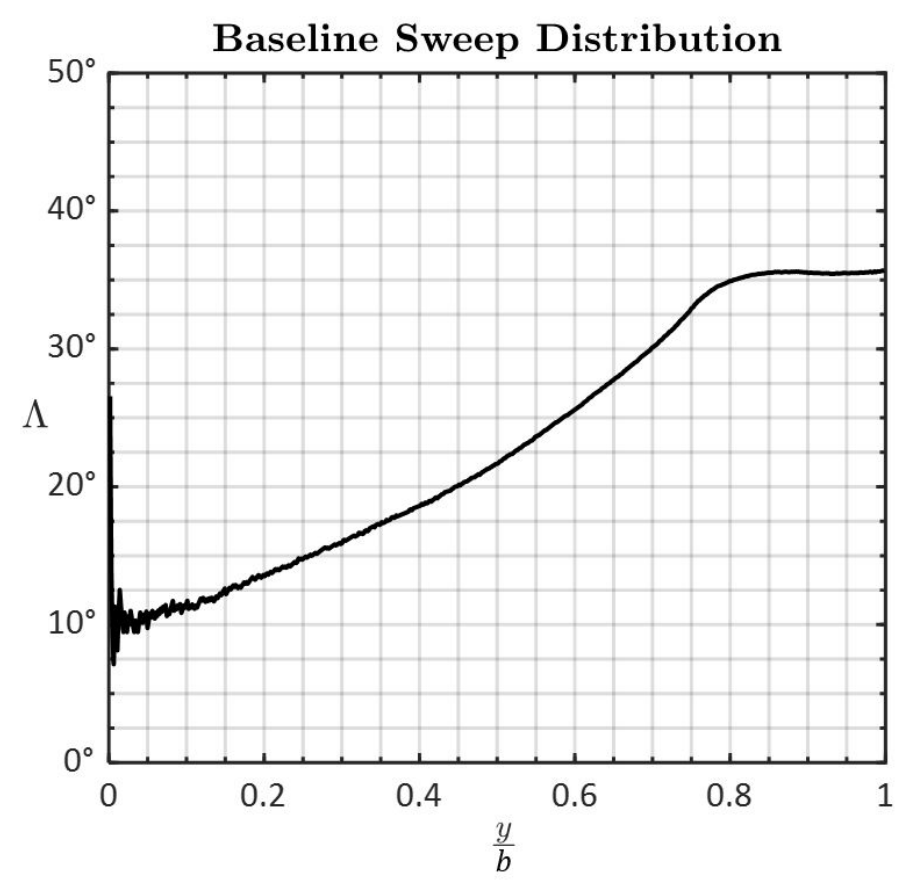

Figure 4.6: Sweep distribution calculated using the centerline created from the baseline fin.

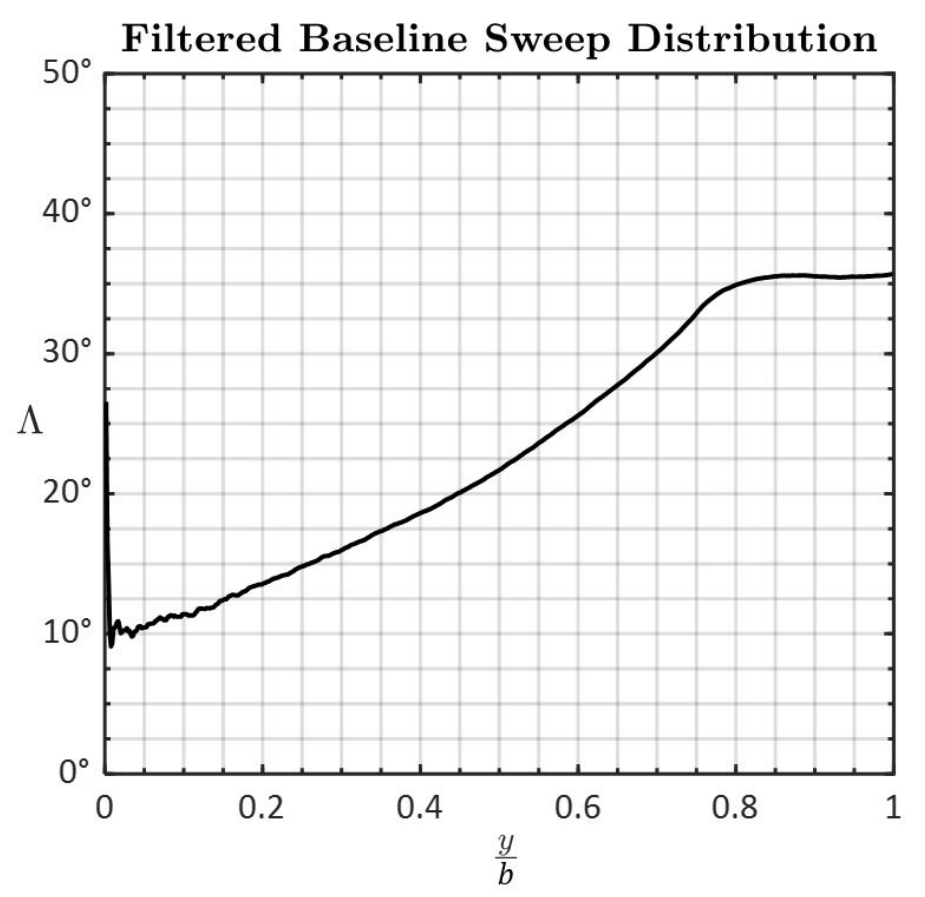

Figure 4.7: Filtered sweep data using a moving average filter. 
It was also possible to fit a continuous line to the sweep distribution using a piecewise polynomial fit. It was difficult to fit a single, continuous polynomial function to the curve, but when split at $\frac{y}{b}=0.75$, two cubic polynomial functions can trace the distribution. Equation 4.7 shows an example piecewise function for the baseline sweep distribution, and a plot for Equation 4.7 can be seen in Figure 4.8. The piecewise function was not used for any calculations, but was proof that the discrepancies in the original distribution can be smoothed out mathematically.

$$
\Lambda\left(\frac{y}{b}\right)= \begin{cases}28.1\left(\frac{y}{b}\right)^{3}-10.4\left(\frac{y}{b}\right)^{2}+23.5\left(\frac{y}{b}\right)+9.05, & 0 \leq \frac{y}{b} \leq 0.75 \\ 763\left(\frac{y}{b}\right)^{3}-2080\left(\frac{y}{b}\right)^{2}+1890\left(\frac{y}{b}\right)-535, & 0.75<\frac{y}{b} \leq 1\end{cases}
$$

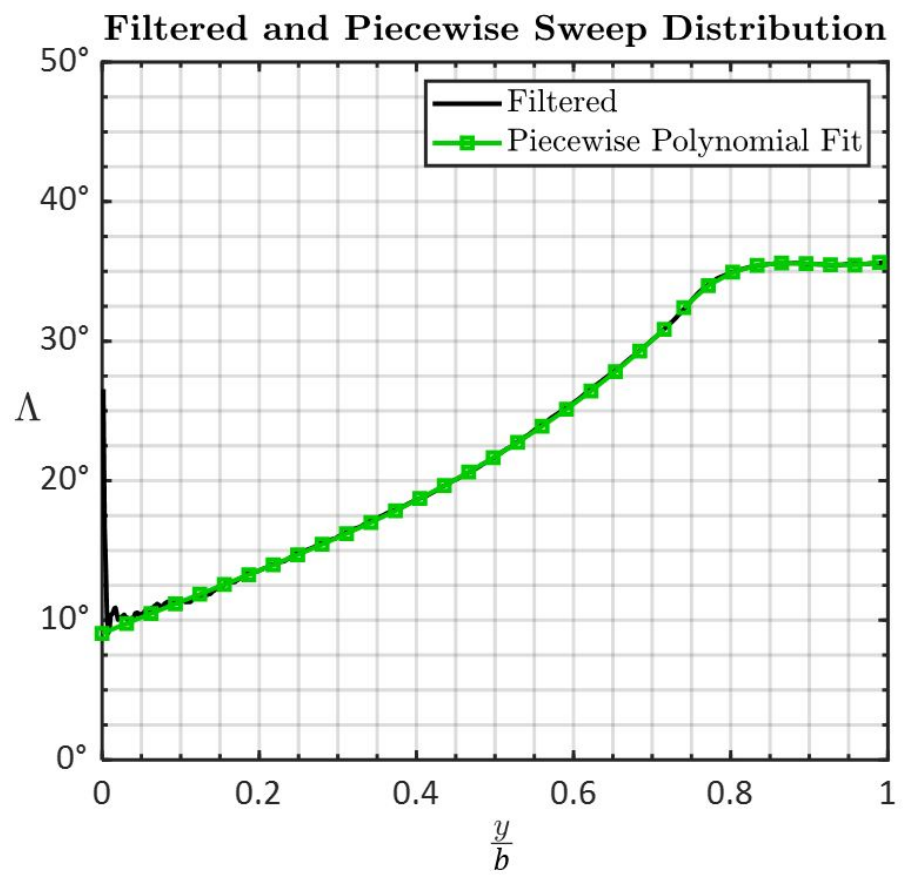

Figure 4.8: Piecewise polynomial function fit to the baseline sweep distribution.

Examining Figure 4.7, there was a gradual increase in $\Lambda(y)$ until $\frac{y}{b} \sim 0.8$, where it plateaus to the final value, $\Lambda_{t i p}=35.2^{\circ}$. Meaning for the baseline shape, the sweep gradually increased until approximately $80 \%$ of the span, where it remains close to 
constant. The FCS II FT was the only baseline shape analyzed here, but it could prove worthwhile in future studies to see if this trend is prevalent in other designs.

\subsubsection{Changing Fin Sweep}

There are only two variables that can be changed in Equation 4.6: $\Delta x$ or $\Delta y$. However, for this given system, $\Delta y$ must remain constant. Changing $\Delta y$ would result in increasing or decreasing the span and aspect ratio of the fin. Therefore, the only option was to change $\Delta x$.

The root chord length and location remained constant, which isolated $x_{c / 2}$, the half chord, $x$ coordinate at a given cross section. To change this, $x_{c / 2}$ was translated proportionally to a desired new $\Lambda_{t i p}$. The new $\Lambda_{t i p}$ value describes the final swept point of the fin, was user chosen, and used to label each of the final geometries tested.

A multiplication constant was calculated using the ratio of original and desired tip sweep angles, where the subscript new denotes a new input $\Lambda_{\text {tip }}$ value, and 0 is the starting tip angle.

$$
K_{\text {sweep }}=\frac{\Lambda_{\text {tip,new }}}{\Lambda_{\text {tip }, 0}}
$$

This constant was multiplied to the original sweep distribution, and the result was a proportionally shifted distribution. The new sweep distribution was used to back out the new half chord location. At a given cross section, the original sweep angle was multiplied by the calculated constant:

$$
\Lambda_{\text {new }}(y)=\Lambda_{0}(y) \cdot K_{\text {sweep }}
$$

$\Lambda_{\text {new }}(y)$ was multiplied by the $y$ value at that location to get the corresponding $x$ value.

$$
x_{n e w}=\Lambda_{n e w}(y) \cdot y
$$

This $x$ value represents a location with respect to the origin. In order to calculate 
the new half chord location, $x_{\text {new }}$ had to be added to the original half root chord.

$$
x_{c / 2, \text { new }}=x_{\text {new }}+x_{r, c / 2}
$$

Once the $x$ coordinate of the half chord was found, the last step was to find the new leading edge and trailing edge point coordinates. This was accomplished by subtracting and adding half the corresponding taper value at the given height.

$$
\begin{aligned}
& x_{\text {LE, new }}=x_{\text {new }}-\frac{\lambda(y)}{2} \\
& x_{\text {TE,new }}=x_{\text {new }}+\frac{\lambda(y)}{2}
\end{aligned}
$$

By using taper values from the baseline distribution, the original taper distribution was maintained. The fin shape did not increase or decrease its span, and the surface area remained constant. The final result was a planform shape that was at new $\Lambda_{t i p}$ angle, with a sweep distribution proportionally translated to the new $\Lambda_{t i p}$.

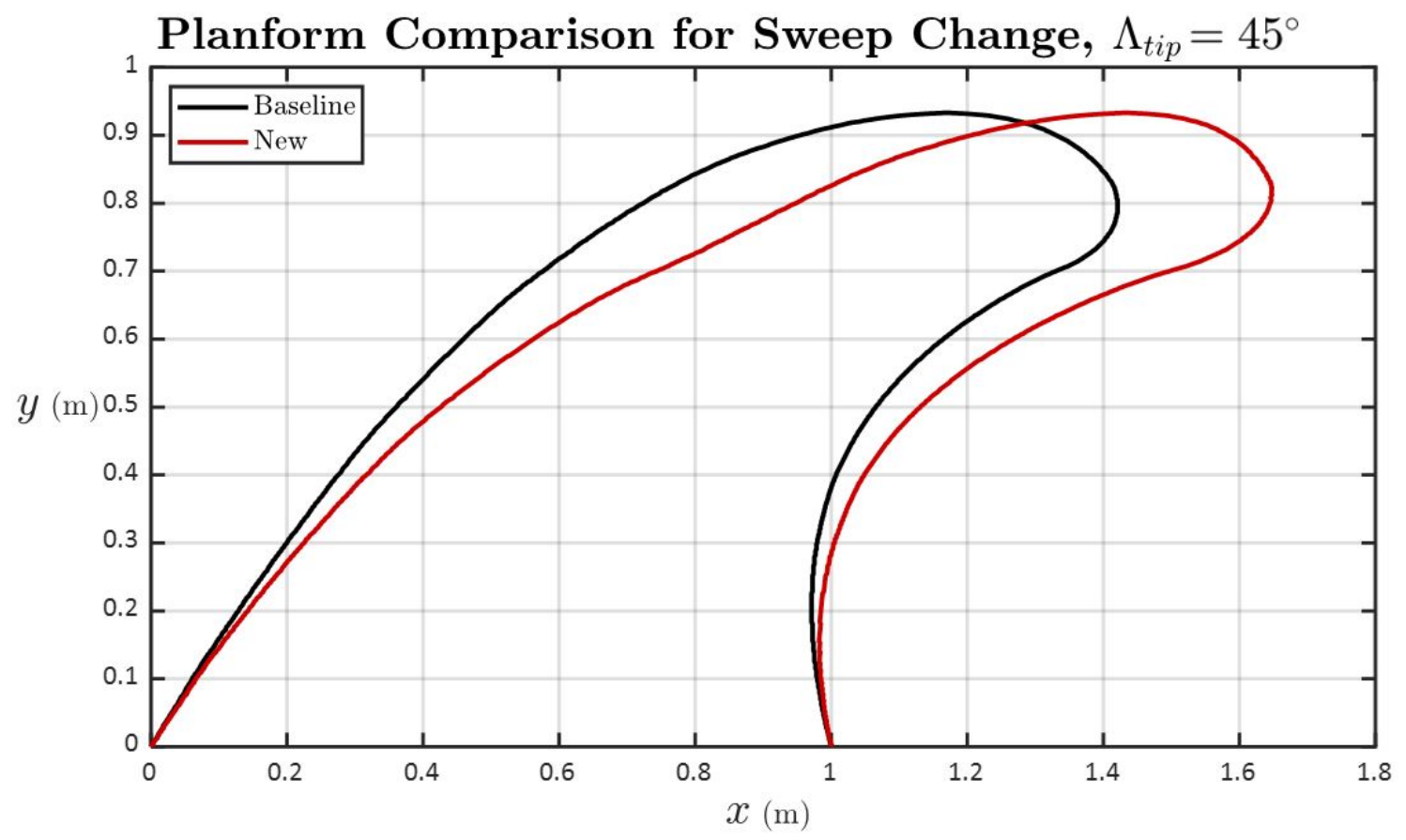

Figure 4.9: Planform outline for both the baseline shape and the new shape with changed sweep distribution.

At first glance, the new fin outline in Figure 4.9 may look to have changed it's taper distribution, but this was not the case. The perimeter of the fin has been elongated, 
but the area has remained the same. To validate this, Figure 4.10 shows the change in sweep distribution and Figure 4.11 shows taper distribution at the same conditions (the taper distribution methodology is shown in the following section). Here the sweep distribution gradually increases until reaching $\Lambda_{t i p, \text { new }}$ and the baseline and new taper lines overlap each other, meaning that the taper distributions are equivalent.

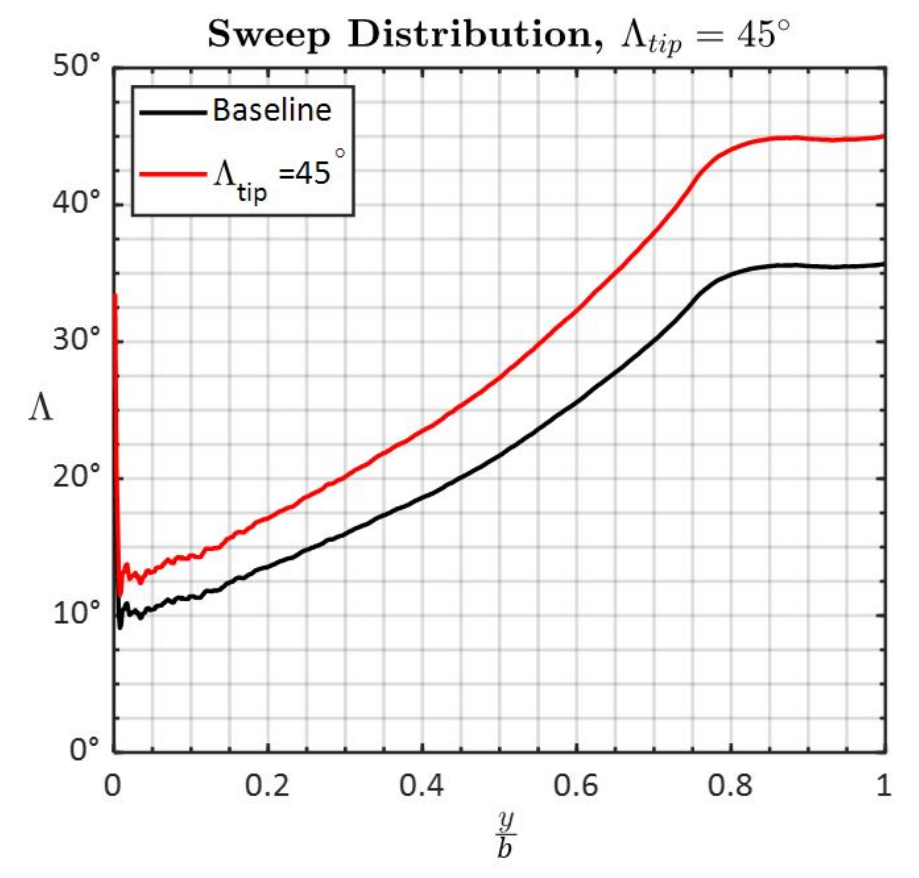

Figure 4.10: Comparison of sweep distributions for $\Lambda_{t i p}=45^{\circ}$.

There was however a noticeable change in concavity on the leading edge. The baseline shape has a uniformly concave leading edge, but the new planform changes at $x \sim 0.8 \mathrm{~m}$. This was a product of the taper distribution. The taper distribution saw an increase and decrease in chord size along the span of the fin, and because of this, the concavity of the fin can change. On the baseline geometry, this is what creates the "S" like curve on the trailing edge. The change in concavity is an issue that should be addressed in follow up research, but did not make any of the new fin shapes invalid. 


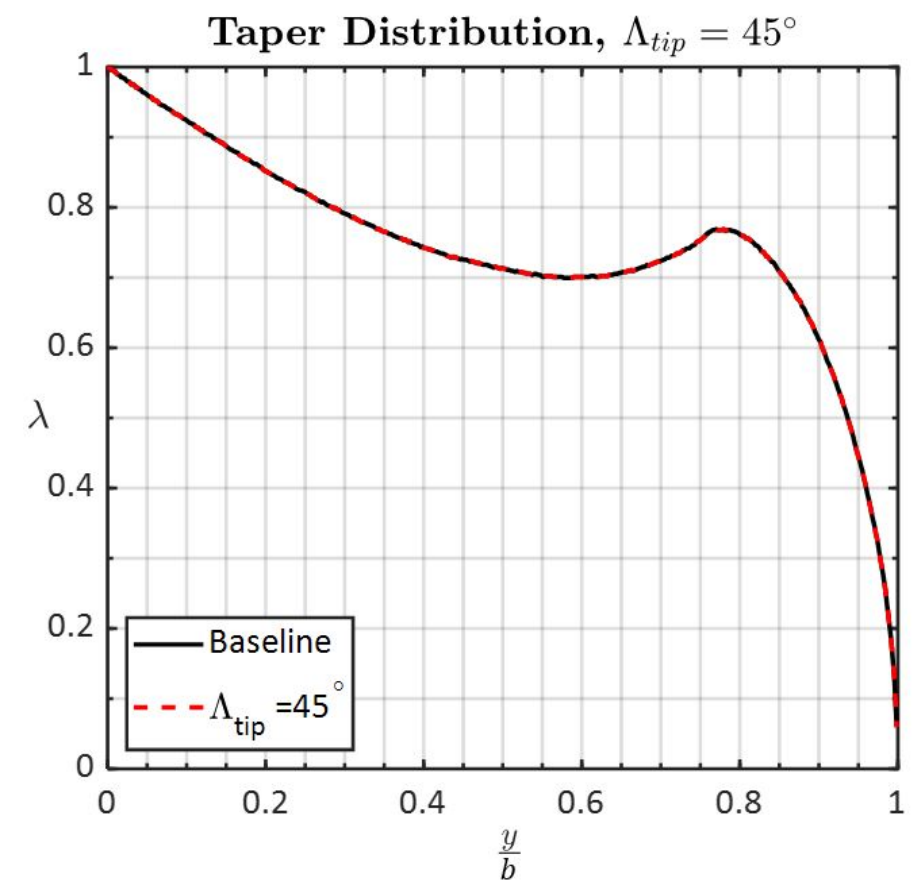

Figure 4.11: Comparison of Taper Distribution for $\Lambda_{t i p}=45^{\circ}$. The lines overlap, meaning the two distributions are equal.

\subsubsection{Calculating Fin Taper}

Similar to the sweep distribution, before the taper distribution was altered, it was first characterized. Using Equation 3.2, where $c_{y}$ is calculated from the leading and trailing edge points at a given $y$ coordinate, the taper can be calculated and plotted with respect to the span of the fin, seen in Figure 4.12.

Figure 4.12 also plots a horizontal line for the $M H C$. The distribution shows that close to the root chord, the taper remains close to one, but as $\frac{y}{b}$ increases, the taper decreases until $\sim 60 \%$ of the span. After this point, the distribution bottoms out and trends oppositely to increasing values. This continues until $\sim 80 \%$ of the span, where afterwards it dramatically decreases to the tip point. This results in two different concavities on the plot: concave up for the first $\sim 80 \%$ and concave down for the ending portion.

This could be a trait of a surfboard fin that gives it a dorsal fin like look. If 


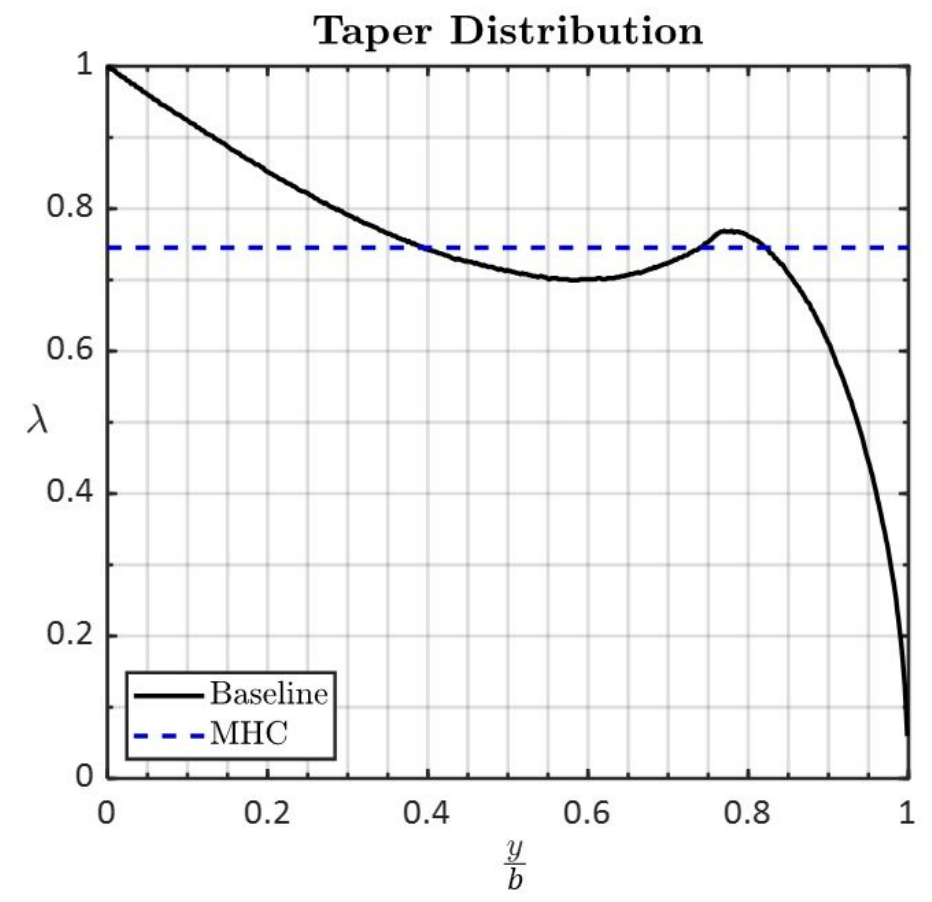

Figure 4.12: Taper distribution for baseline fin geometry along with a line illustrating the mean hydrodynamic chord.

the local taper continued to decrease towards the the tip, the planform shape might represent an elliptical or trapezoidal wing depending on the rate at which the chord length decreased. However, since there was an increase of chord length towards the tip, the fin maintains its surface area a little longer.

For the baseline fin, this also meant that the $M H C$ was at three separate locations: once at the initial decrease and then twice where concavity changes. It is not appropriate to say this applies to all surfboard fin geometry without further testing, but it can be suggested that other fins share this feature.

\subsubsection{Changing Fin Taper}

The fin taper was not changed as simply as the sweep. For the fin sweep, all that was applied was a proportionality constant to offset the sweep whilst maintaining span and area. The sweep did not directly influence the calculation of area, because the 
chord length and $y$ coordinates were maintained throughout any changes. However by definition, in order to change the taper distribution the chord lengths had to change. If the taper distribution was multiplied by a constant, the entire distribution will either increase or decrease, and consequentially change the surface area accordingly.

$$
\lambda_{\text {new }}(y)=\lambda(y) \cdot K_{\text {taper }}
$$

While the results from applying this kind of modification are not comparable, four simulations were run for thoroughness applying Equation 4.14. The results from these simulations can be seen in Appendix B.4.

The challenge with changing the taper was that there had to be an equal amount of increase and decrease in surface area. Meaning, the integral of the change in area had to equal zero. The perimeter of the fin needed to change non-uniformly while the area remained constant. At the time of this research, attempts to model this by equation proved exceedingly difficult and another method was sought.

An alternative solution was found, within a set of limitations. The requirements for the solution were:

- The $M H C$ remained constant.

- The new surface area had to be within $5 \%$ of the starting surface area.

The priority was to maintain the $M H C$ in order to maintain Reynolds Number similarity and to provide an inflection point. There needed to be a point where the chord lengths switched from decreasing to increasing in size so that the surface area was allowed to change appropriately. The $M H C$ was chosen to be this point. Through this thought process Equation 4.15 was constructed.

$$
\lambda_{\text {new }}(y)=\lambda(y)-K_{\text {taper }} \cdot \lambda(y)(M H C-\lambda(y))
$$


The logic behind this equation is as follows. To calculate $\lambda_{\text {new }}$, the original taper value, $\lambda(y)$ was either increased or decreased by the product of three values, seen on the right hand side of Equation 4.15. One of these values is the quantity of $(M H C-\lambda(y))$. As mentioned, $M H C$ was an inflection point in the taper distribution. When the original taper value equals the $M H C, \lambda(y)=M H C$, the right hand side of the equation goes to 0 , and $\lambda_{\text {new }}=M H C$. This maintained the $M H C$ and satisfied the first requirement. When $\lambda(y) \neq M H C$, the result was either a positive or negative number depending on whether or not the chord length was smaller or bigger than the $M H C$. Therefore, the $M H C$ remained constant, and the chord length increased or decreased around it.

$K_{\text {taper }}$ was a user set constant. It was used as a method for controlling the scale and the sign of the right hand side of the equation and to differentiate and generate the planforms.

The last term of the product was $\lambda(y)$, the original taper value. By including $\lambda(y)$ in the product, the change in taper values was scaled by the taper at that location; small chord lengths resulted in smaller changes in taper. If $\lambda(y)$ was not included in the product, there was a point where the subtraction of $M H C-\lambda(y)$ was to great, and the leading and trailing edge offset past each other. This issue is highlighted in Figure 4.13.

Including $\lambda(y)$ in the product, however, made Equation 4.15 non-linear, and as a result, the change in area between the new and old taper distributions can become non-zero. In future applications, this needs to be addressed, but for the purpose of this research, this was a non-issue when $-1<K_{\text {taper }}<1$. $K_{\text {taper }}$ values outside this range resulted in a change of surface area more than $5 \%$.

Figure 4.14 and Figure 4.15 show an application of Equation 4.15 to create a new fin planform and taper distribution with $K_{\text {taper }}=0.75$. The planform comparison 


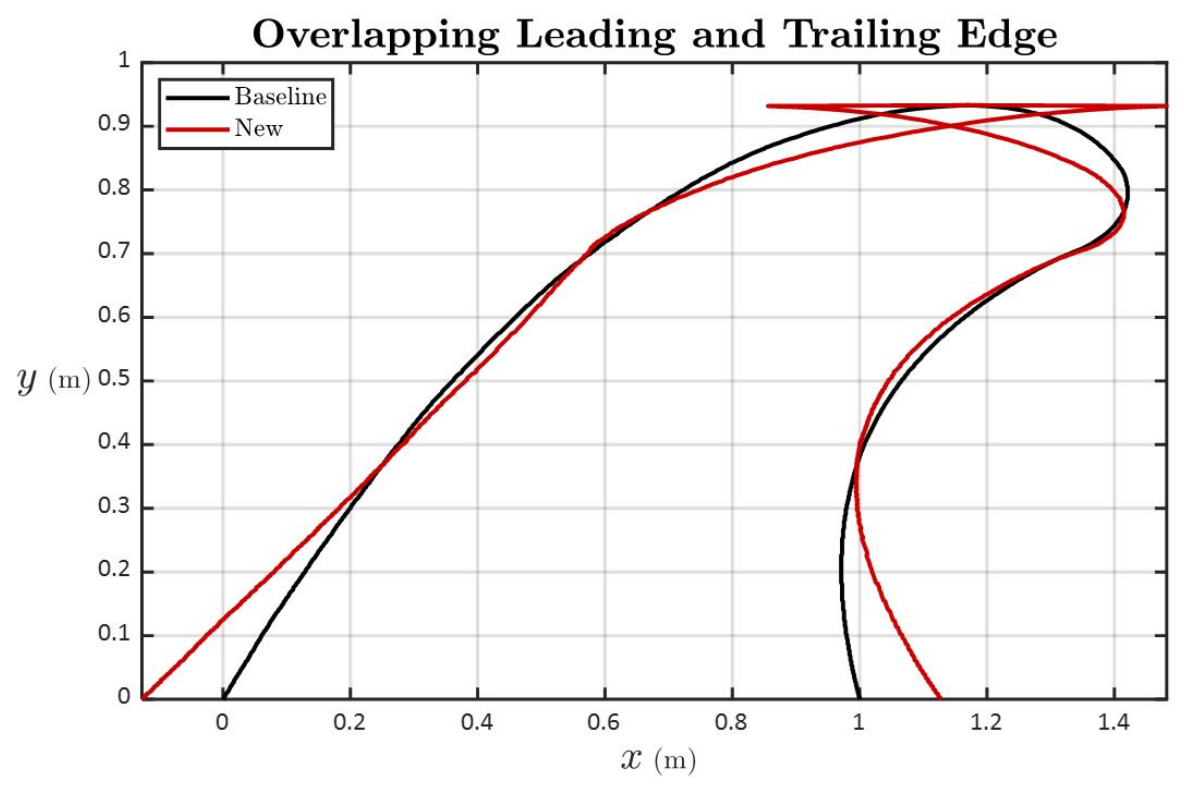

Figure 4.13: The leading and trailing edge overlapping before the fin tip.

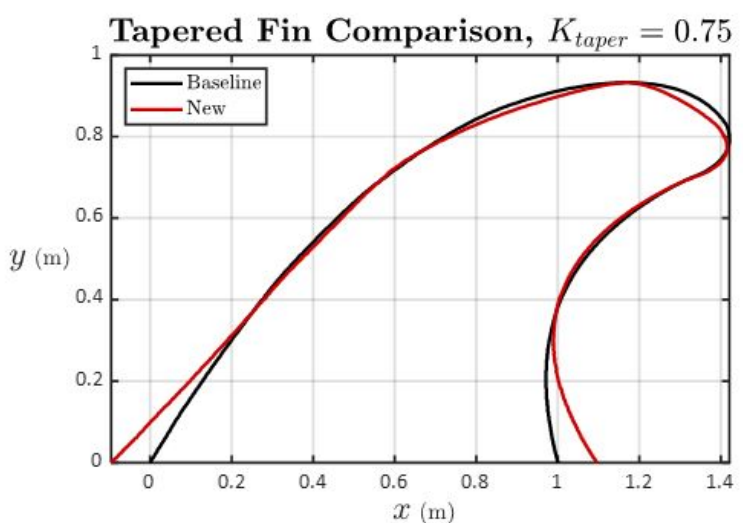

(a)

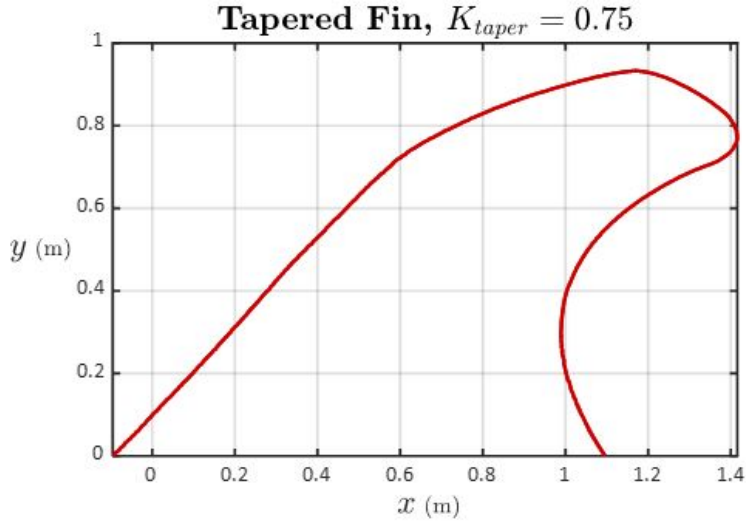

(b)

Figure 4.14: Comparison of baseline shape with newly tapered fin after applying Equation 4.15 and $K_{\text {taper }}=0.75$. (a) Contains both the outline of the new shape along with the baseline fin. (b) The new fin outline.

illustrates an increase in the root chord and a decrease in area close to the tip, and the $M H C$ remains constant for both shapes. When $K_{\text {taper }}$ was positive, the decrease in chord size closer to the fin tip was accelerated, increasing the sharpness. The opposite was true when $K_{\text {taper }}$ was negative, the root chord decreased in size and the tip became more rounded. 


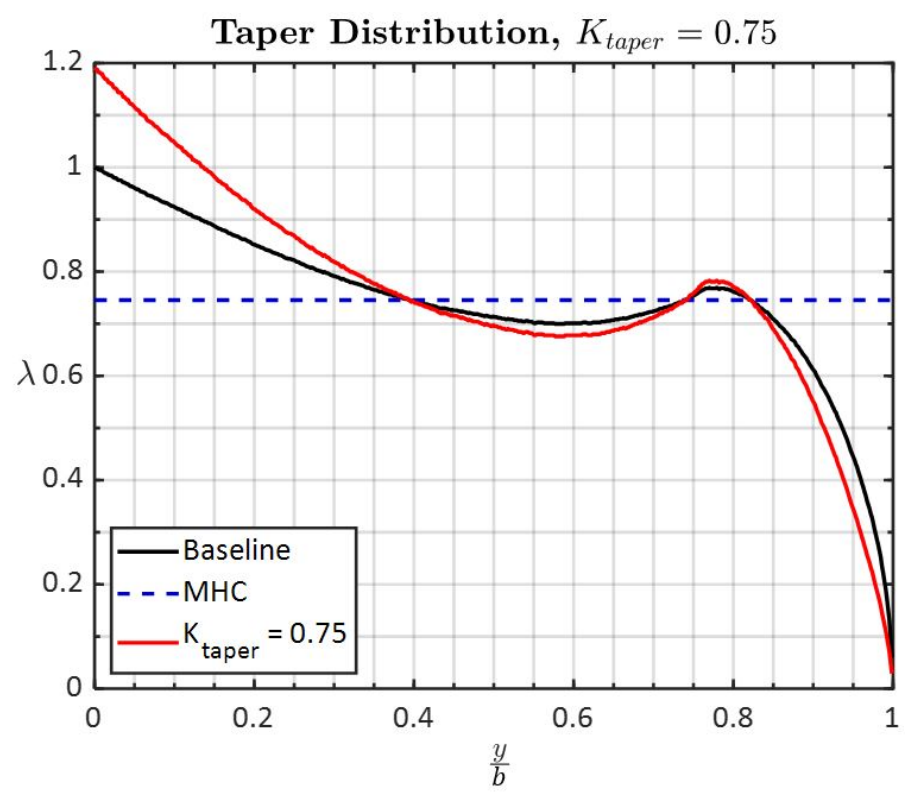

Figure 4.15: Taper distribution comparison for $K_{\text {taper }}=0.75$. Note that the the distributions for both the new and baseline lines overlap at the MAC.

These figures also show the slight difference in area change. There were six different $K_{\text {taper }}$ values that were tested, shown in Table 4.3. The greatest increase in surface area was when $K_{\text {taper }}=-1.00$ at $2.81 \%$, which was a small enough percentage to be considered an acceptable change.

Table 4.3: Comparison of root chord and surface area for different tapered fins. $K_{\text {taper }}=0$ is the baseline geometry.

\begin{tabular}{c|ccccccc}
\hline$K_{\text {taper }}$ & $\mathbf{- 1 . 0 0}$ & $\mathbf{- 0 . 7 5}$ & $\mathbf{- 0 . 5 0}$ & $\mathbf{0 . 0 0}$ & $\mathbf{0 . 5 0}$ & $\mathbf{0 . 7 5}$ & $\mathbf{1 . 0 0}$ \\
\hline$c_{r}(\mathbf{m})$ & 0.745 & 0.809 & 0.872 & 1.00 & 1.13 & 1.19 & 1.25 \\
\hline$S\left(\mathbf{m}^{2}\right)$ & 0.675 & 0.680 & 0.685 & 0.695 & 0.704 & 0.709 & 0.714 \\
\hline Percent Difference $S$ & $2.81 \%$ & $2.01 \%$ & $1.39 \%$ & $0.00 \%$ & $1.37 \%$ & $2.06 \%$ & $2.73 \%$ \\
\hline
\end{tabular}

Using this method, the centerline of the fin did not change, and $\Lambda_{\text {tip }}$ remained constant at $35^{\circ}$, shown in Figure 4.16. Therefore, the taper distribution was the only variable changed. 


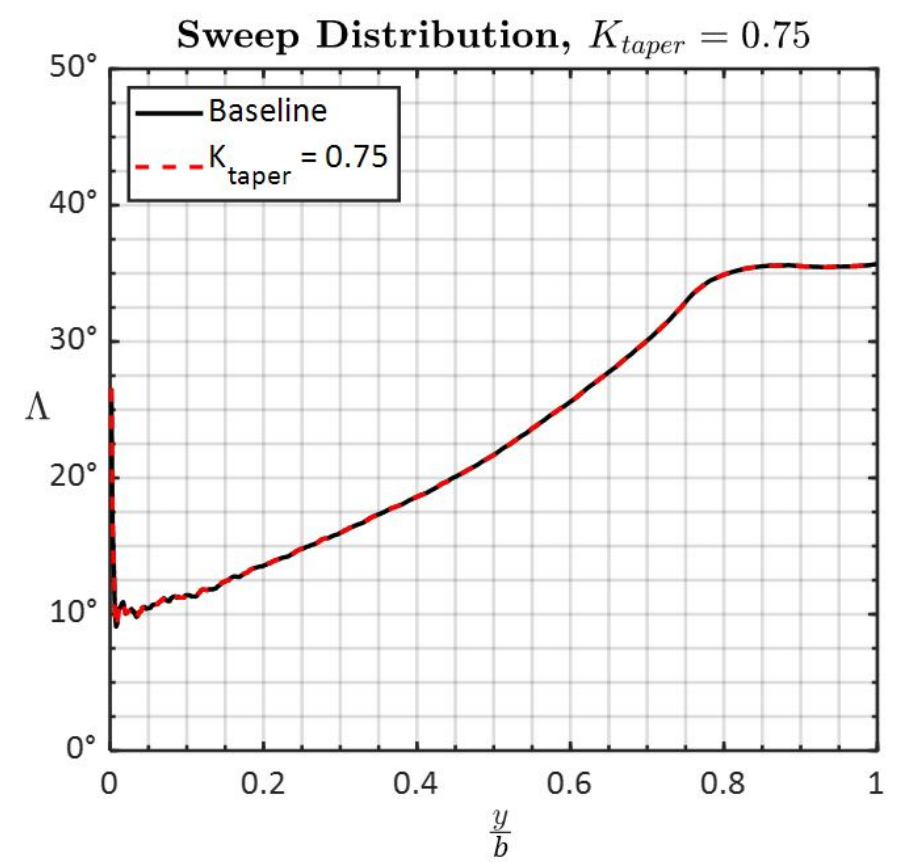

Figure 4.16: Sweep distribution comparison for $K_{\text {taper }}=0.75$. The red dashed line and black line perfectly overlap, illustrated equal sweep distributions.

\subsection{CAD Creation}

After both the changes in sweep and taper were defined, the final step to create the three dimensional models was to export the modified leading and trailing edge points into Solidworks and loft together the model using the NACA 0006. The leading and trailing edge were exported as two-column comma separated values files (.csv) with $x$ and $y$ coordinates, and a third column filled with 0's for the $z$ coordinate were added after the original export. The points were converted to a text file (.txt) and imported into Solidworks using the Curve Through XYZ Points tool along with the coordinates for the NACA 0006 hydrofoil.

The NACA 0006 points were converted from a curve to a sketch feature on the $\mathrm{XY}$ plane, and then the leading and trailing edge were translated to be coincident to the leading and trailing point on the hydrofoil. 
The lofting operation worked by taking a starting and ending sketch as inputs and then blending the two shapes together in three dimensional space using guide curves to define the edges. For a surfboard fin, the two sketch inputs would be the hydrofoil at the root of the fin and the point at the tip, and the guide curves would be the leading and trailing edge. The point at the tip, however, was an issue for the lofting operation. The hydrofoil was forced to blend to a single point, and as result, the cross sections would begin to lose the hydrofoil shape and collapse. This creates inconsistent and uncharacterized cross sections. To correct for this, the point at the tip was replaced with a second NACA 0006 hydrofoil and was fit to be the width of the last leading and trailing edge points in the $y$ direction. In doing so, the NACA 0006 cross section was maintained from the root to the tip of the fin.
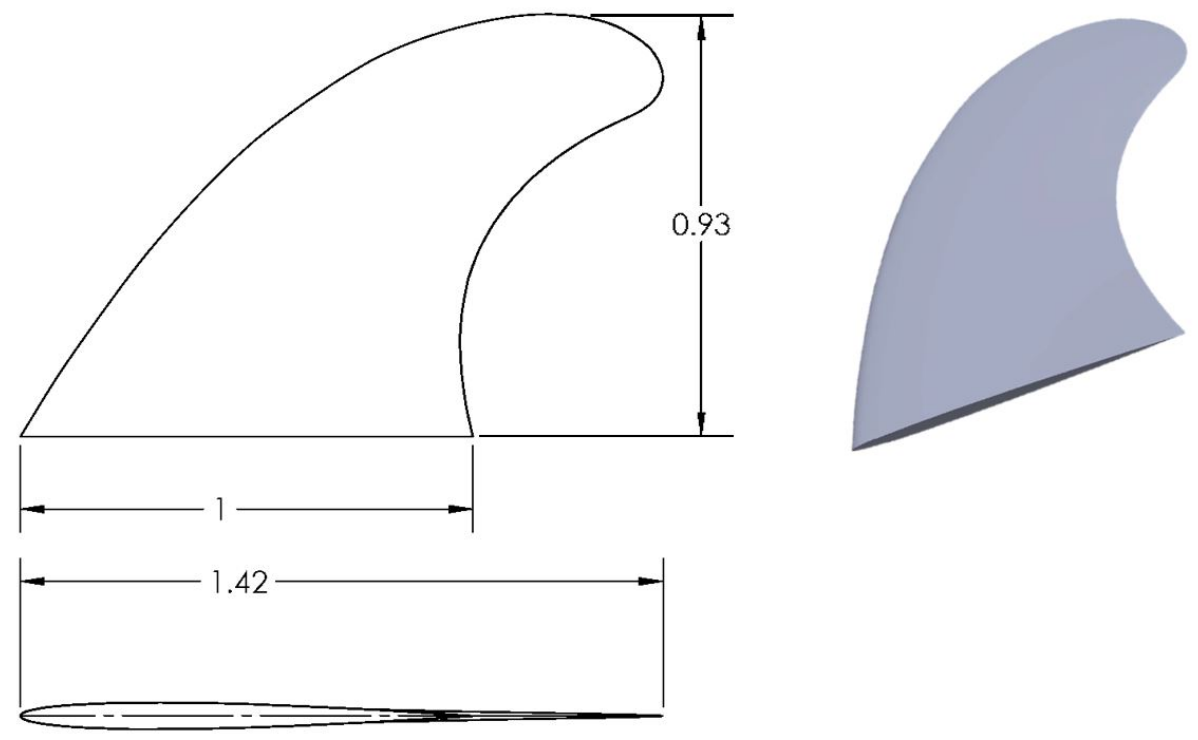

Figure 4.17: Two view drawing of baseline fin with included isometric view. Units are in meters.

The reason for this decision was to maintain consistency for all geometries. If the tip point was used, there is no user control of how and where the airfoil begins to blend, resulting in inconsistent cross sections. Figure 4.18 shows a zoomed in perspective of the fin tip hydrofoil used in the base geometry with a chord length of $10.41 \mathrm{~mm}$. This was approximately $1 \%$ of the root chord length and was too 
small to make noticeable differences. This methodology was used to create all of the geometries tested in this study.

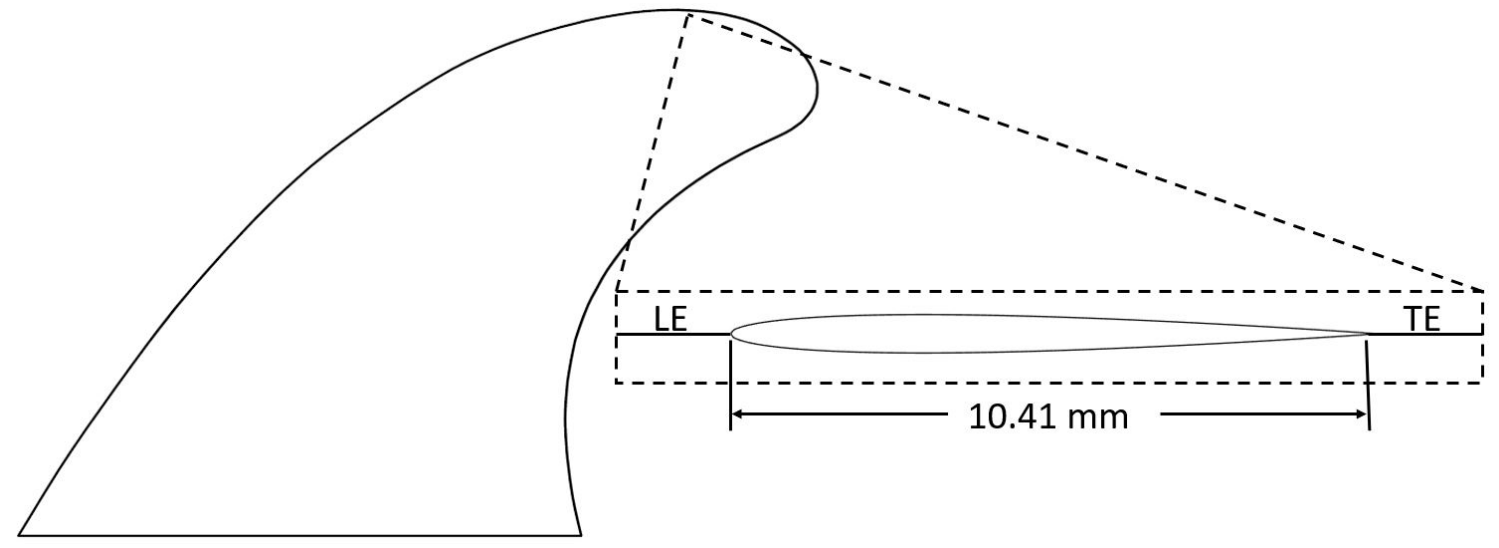

Figure 4.18: Zoomed in perspective of the fin tip hydrofoil used in the base geometry with a chord length of $10.41 \mathrm{~mm}$. The hydrofoil connected the leading and trailing edge at the tip. 
Chapter 5

SIMULATION SETUP

\subsection{CFD Software and Resources}

The CFD simulations were all meshed, solved, and partly post processed in STAR$\mathrm{CCM}+$ version 12.06.011. The CAD was generated in Solidworks and then exported as a parasolid file type into STAR-CCM+. The scalar images and data points were all processed in STAR-CCM+, and any exported data points were manipulated in Matlab.

The domain sizing, grid independence simulations, and final geometry simulations were all run on Cal Poly San Luis Obispo's Bishop High Performance Computer Cluster (Bishop Cluster). The Bishop Cluster is a high performance cluster that features four nodes with 240 processing cores and 1.1 terabytes of ram.

\subsection{Physics Continua}

The physics continua defined the flow field environment of the CFD simulation and was set using the fluid properties of liquid salt water. At $15{ }^{\circ} \mathrm{C}$, the density of salt water is $\rho=1026 \mathrm{~kg} \mathrm{~m}^{-3}$ and incompressible, and the dynamic viscosity is $\mu=1.23 \times 10^{-3} \mathrm{~N} \mathrm{~s} \mathrm{~m}^{-2}[26]$. To determine the freestream velocity of the simulation, Reynolds number matching was used from collected surfer data. As mentioned in Section 1.4.1, the average speed of a surfer was measured at $5 \mathrm{~m} \mathrm{~s}^{-1}$. Using the unscaled $M H C$ of $85.7 \mathrm{~mm}$, the Reynolds Number can be calculated as $R e=3.51 \times 10^{5}$. This Reynolds number was then used and matched to find the freestream velocity for the 
scaled, CAD geometry. Equation 1.2 can be rewritten so that it solves for $U_{\infty}$.

$$
U_{\infty}=\frac{R e \rho l}{\mu}
$$

Using this equation and the $M H C$ of the CAD geometry, $M H C=0.745 \mathrm{~m}, U_{\infty}=$ $0.56 \mathrm{~m} \mathrm{~s}^{-1}$. This would be the velocity magnitude used for all CFD simulations.

The steady RANS equations with segregated flow were used for the solver. The flow field around a surfboard fin is rarely steady, or invariant with time, due to the constantly changing wave shape and surface composition, but using unsteady equations to capture this requires much more computational power and time. Currently, there is also no validated estimation of what the unsteady flow field for a surfboard fin is either, so the increased resource usage was not justified.

The segregated flow model was chosen over coupled as it solves each of the momentum equations in turn; opposed to a coupled flow model that solves mass, momentum, and energy simultaneously [28]. The coupled model can help with convergence for compressible fluids but was not necessary for improving the convergence of the physics modeled.

The realizable $k-\epsilon$ model was used for the turbulence model with a two layer all wall $\mathrm{y}+$ treatment. The $k-\epsilon$ series of turbulence models are two equation turbulence models with an equation for the turbulent kinetic energy, $k$, and the dissipation rate, $\epsilon$ [28]. The realizable version of $k-\epsilon$ uses a revised version of the transport equations that provide improved predications of separation, boundary layers, and round jets, which was more useful for capturing the separation and vortex formation around the fins. The two layer treatment allows the user to apply fine meshes close to the wall, is done to better capture viscosity near the wall, but must have the correct wall $y+$ value, described in subsection 5.4.1. 


\subsection{Fluid Domain}

The fluid domain is the confined volume or area that is meshed and solved for by the CFD software. It has defined outer boundaries that limit where the flow field is solved for and was setup to be a rectangular prism with two different boundary settings. The first set of boundary conditions were used when the incidence angle, $\alpha_{F}$, was equal to zero. For this condition, the flow enters from a single velocity inlet boundary and exits through a single pressure outlet. The second set of conditions were setup for when $\alpha_{F} \neq 0$. Instead of rotating the fin geometry to meet the changing incidence angle, the direction of the velocity vector was instead set to equal $\alpha_{F}$. By doing so, the geometry did not have to be rotated and re-meshed for every change in $\alpha_{F}$. Instead, a pair of side walls were changed to an inlet and outlet pair so that the freestream could enter and exit the domain at an angle. Figure 5.1 illustrate these two setups from a top down perspective.

Figure 5.1 also has color coding for the shear stress specification. There is no boundary layer growth until the water first encounters the surfboard. At this point, the boundary layer develops through the end of the surfboard where it returns to freestream slip conditions. This is a defining characteristic of the flow field around a surfboard fin, so to loosely approximate this, the intersecting surface was split into three surfaces, as seen in Figure 5.2.

As mentioned previously, modeling the shape and submersion level of the surfboard bottom adds complexity outside the scope of this research. Modeling the surface correctly would increase the applicability of the simulations, but without having an established, validated method for doing so, there is not yet justification for it. Therefore, the no-slip surface was assumed to be continuous with the slip surface and spanned the width of the domain. 


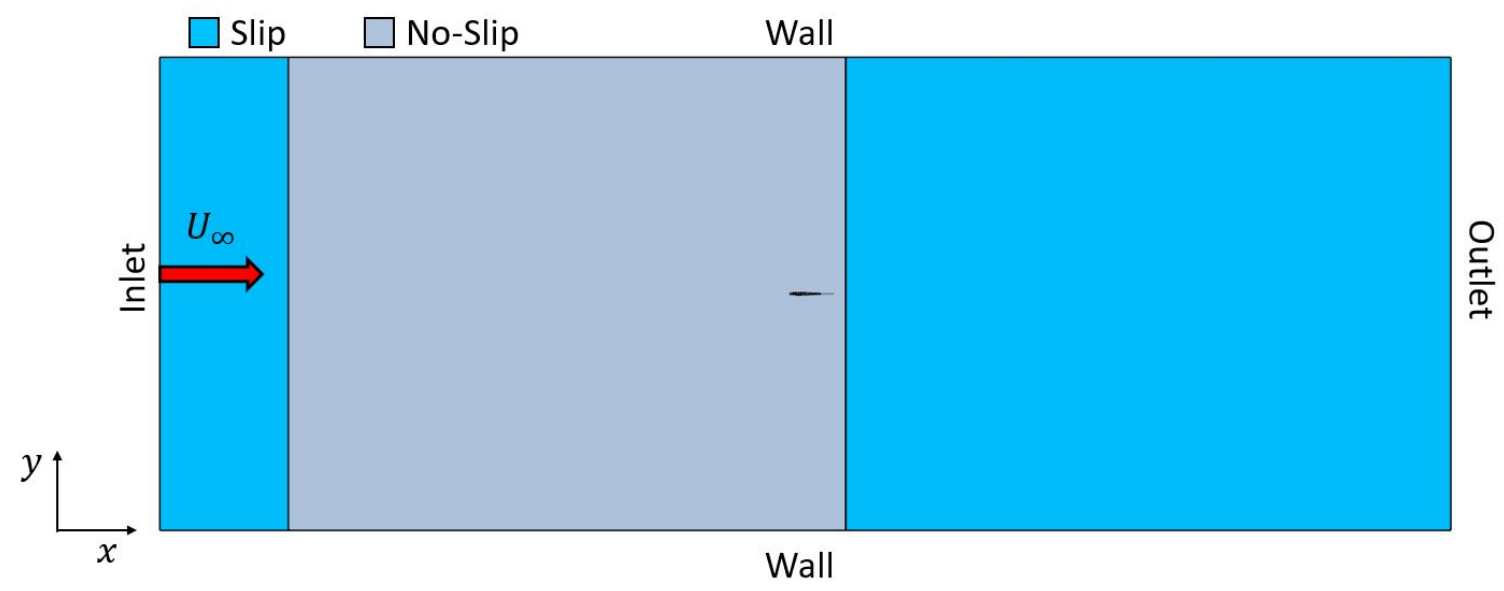

(a) $\alpha_{F}=0$

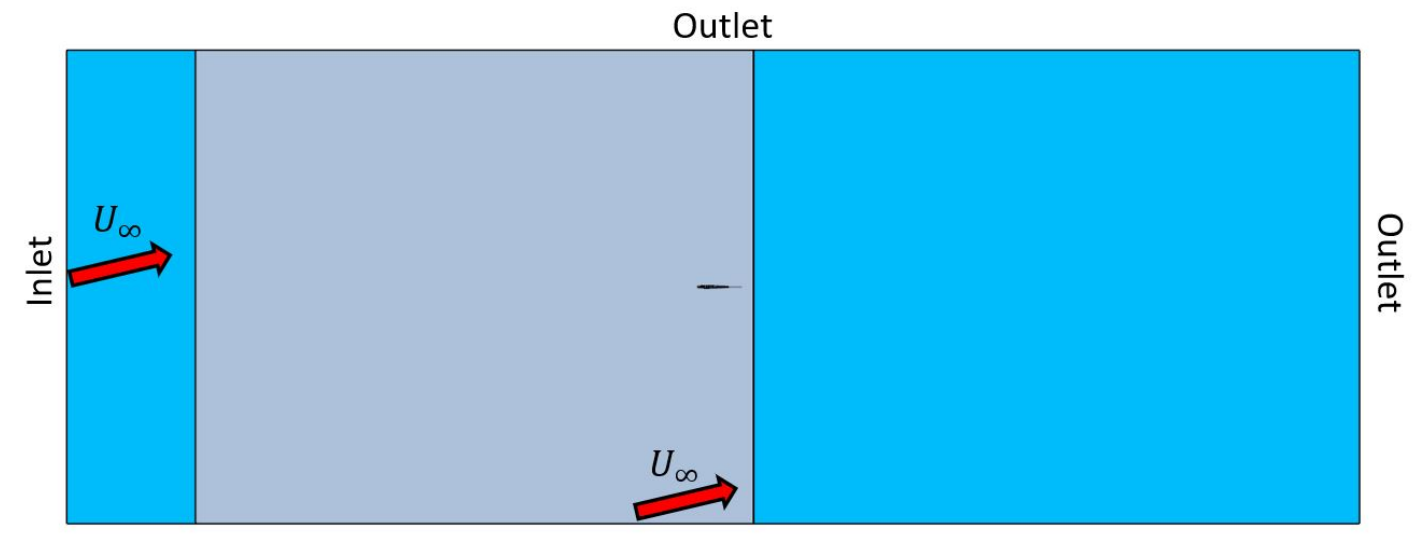

Inlet

(b) $\alpha_{F} \neq 0$

Figure 5.1: Two different boundary settings used depending on the incidence angle

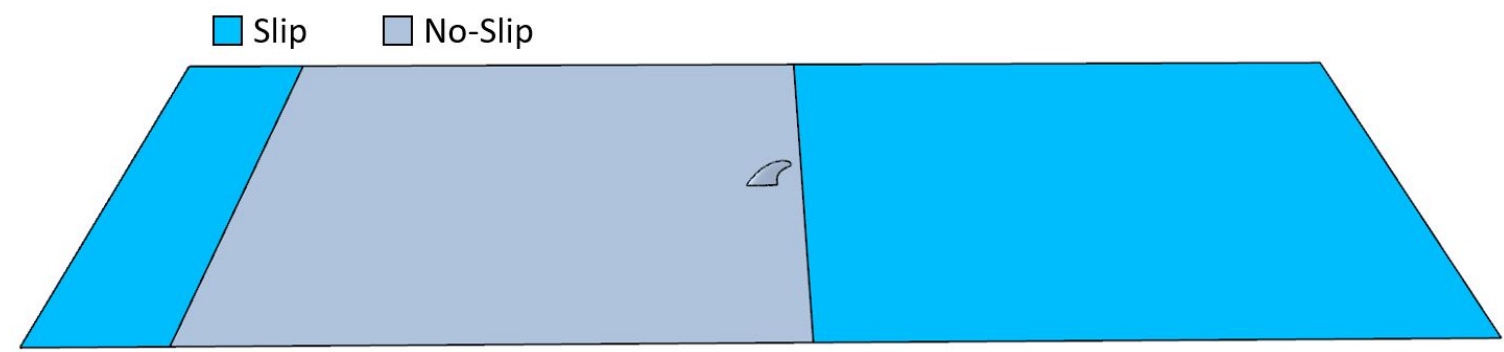

Figure 5.2: Fluid domain bottom boundary split into three separate surfaces. The blue indicates the ocean surface where there is no boundary layer growth, and the grey indicates no-slip surfaces where the boundary layer is present. Freestream is going from left to right. 


\subsubsection{Fin Placement}

The surfboard fin geometries were imported into STAR-CCM+ as three dimensional models, and translated to sit flush on the no-slip boundary proportional to the fin placement on a standard surfboard. Using the dimensions for a $2.0 \mathrm{~m}$ surfboard, a shaper in San Diego has quoted that the center fin of a thruster configuration sits $89.9 \mathrm{~mm}$ (3.5 in) from the tail of the board [29]. Using this as reference and scaled proportionally to the size of the baseline geometry, the fin in the domain was offset $787 \mathrm{~mm}$ from the tail of the no-slip boundary, and the no slip boundary was scaled to have a total length of $17.7 \mathrm{~m}$.

\subsubsection{Fluid Domain Sizing}

The rest of the boundary lengths were sized using a domain sizing study. This was done by generating an unstructed mesh, and solving the simulation at $\alpha_{F}=12^{\circ}$ with varying domain sizes. The mesh settings were not the final settings used, as those were determined after the domain sizing. Instead, estimated settings were used, seen in Table 5.1, and the simulations were iterated until each of the residuals built into the software went below $1.0 \times 10^{-3}$ after at least 1,500 iterations. The meaning and significance of the settings in Table 5.1 are explained further in Section 5.4, and on average, the cell count for each of the domain sizes was close to two million cells.

Table 5.1: Mesh settings used for the domain sizing study. The sizes refer to the target cell size for the given setting.

\begin{tabular}{cccc} 
Fin Surface Size & Domain Size & Wake Size & Growth Rate \\
\hline 0.0075 & 0.25 & 0.02 & 1.2 \\
\hline Prism Layer Height & Number of Prism Layers \\
\hline \multicolumn{2}{c}{0.0005} & 3 \\
\hline
\end{tabular}


For each domain size, both the side force and drag were reported on the fin geometry. When there was no significant change in the forces between two different domain sizes, then the domain size was no longer influencing the results generated. Here a significant change is defined as less than $5 \%$ for a percent difference between two sizes.

This was done at $\alpha_{F}=12^{\circ}$ in order to create a wake behind the fin. The final simulations were run up to $\alpha_{F}=20^{\circ}$, but at this angle there was a strong chance there would be separated flow. Separation is difficult for most solvers to converge without a properly sized mesh, so to avoid the risk of non-convergence, a lower incidence angle was used instead. At $\alpha_{F}=12^{\circ}$, there was still a noticeable pressure gradient created and any overly constrained domain walls would influence the oncoming flow and the wake dissipation.

The distance from each wall to the fin was referenced from the leading edge of the root chord, and was spaced by increments of the root chord. The surface that was coincident with the fin was labeled the bottom surface, and the opposite, parallel surface was labeled as the top. The remaining four surfaces used the naming convention in Figure 5.1a. There were a total of three domain sizes used; there dimensions are summarized in Table 5.2.

Table 5.2: Summary of the three domain sizes. The distances are an increments of $c_{r}=1.0 \mathrm{~m}$.

\begin{tabular}{ccccc}
\hline Domain Size & Inlet & Oulet & Top & Walls \\
\hline Small & 17.5 & 15.0 & 3.00 & 10.0 \\
\hline Medium & 20.0 & 20.0 & 7.50 & 15.0 \\
\hline Large & 30.0 & 30.0 & 10.0 & 20.0 \\
\hline
\end{tabular}

The results of these simulations are presented in Table 5.3; the small domain size was the first simulation run and is why it does not have values for percent differences. 
The percent difference of $C_{L_{F}}$ was below one, and the percent difference of $C_{D}$ for the medium and large domain sizes was $\sim 3 \%$ for each case. This means that when the domain size was increased, the body forces on the fin changed slightly and did not effect the results significantly. The medium size was chosen over the small size for the final fluid domain, even though the percent values were similar, to make sure there was no influence from the boundaries without increasing cell count unnecessarily. A three view drawing of the final fluid domain with the baseline fin is shown in Figure 5.3.

Table 5.3: Results of the domain sizing study. Percent Difference is abbreviated as \% Diff.

\begin{tabular}{c|c|c|c|c|}
\hline Domain Size & $C_{L_{F}}$ & \% Diff. $C_{L_{F}}$ & $C_{D}$ & \% Diff. $C_{D}$ \\
\hline Small & 0.554 & & 0.0356 & \\
\hline Medium & 0.550 & $0.70 \%$ & 0.0366 & $2.77 \%$ \\
\hline Large & 0.547 & $0.59 \%$ & 0.0356 & $2.57 \%$ \\
\hline
\end{tabular}
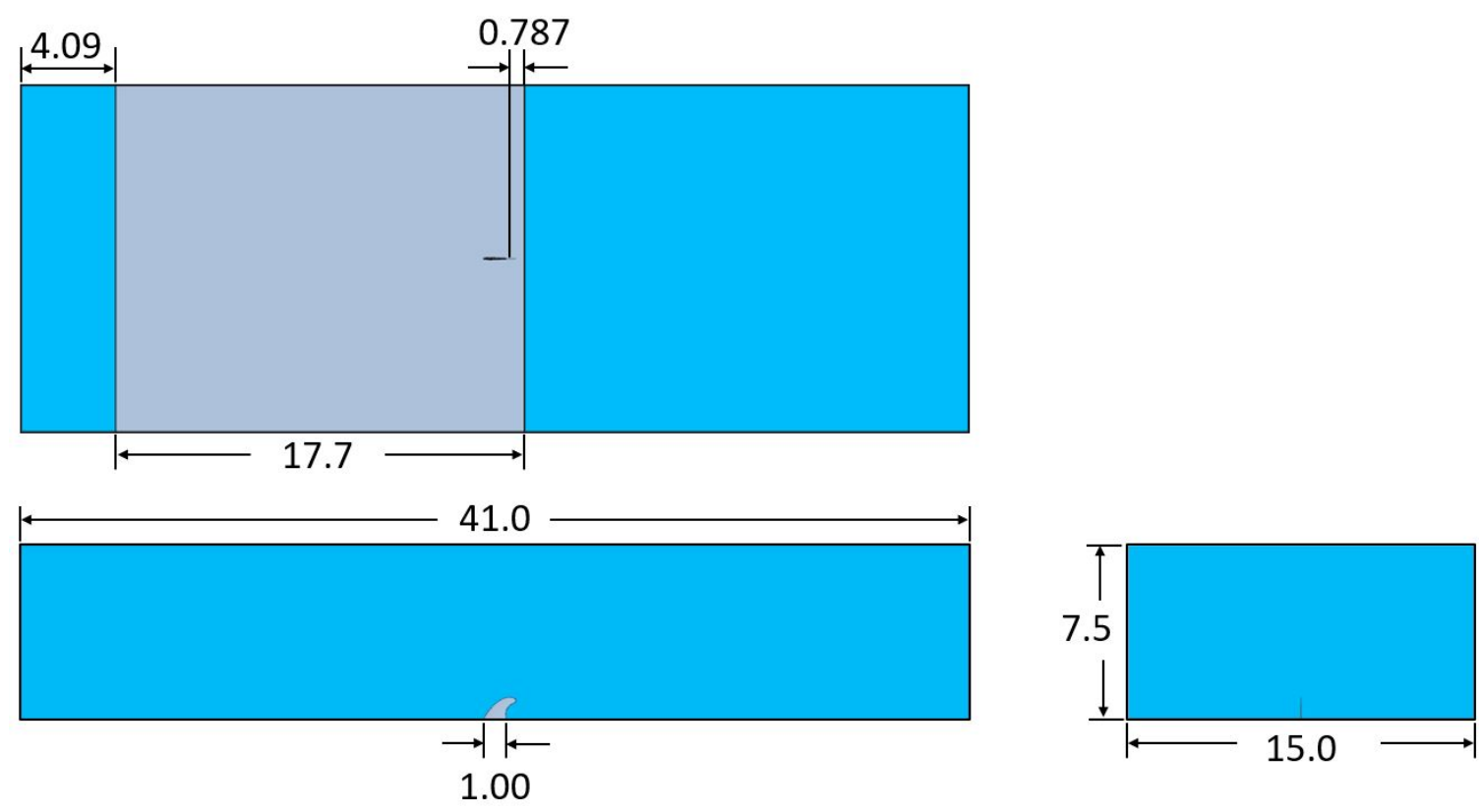

Figure 5.3: Three view drawing of the fluid domain. Grey is a no slip boundary, and the blue is for slip boundaries. All dimensions are in increments of $c_{r}=1.0 \mathrm{~m}$. 


\subsection{Mesh Generation}

Once the domain had been properly sized, a mesh was generated for for the geometry. The mesh for the fin was unstructured, polyhedral and the settings were the same for each incidence angle. Instead of rotating the fin geometry to the incidence angle and re-meshing, the freestream direction was changed. This saved simulation time, but required a grid with a large wake refinement that captured the higher incidence angles. In doing so, the lower incidence angles had more refinement than necessary. The polyhedral mesh was used for it's robustness and low cell count.

\subsubsection{Wall Y+}

In order to capture the boundary layer properly, the wall $y+$ of the generated mesh had to be appropriate to the turbulence model. Wall $y+$ is a non-dimensional distance that relates the cell height away from the wall with the viscosity. It can be calculated using Equation 5.2, where $u_{*}$ is friction velocity, $\mathrm{y}$ is the distance away, and $\nu$ is the dynamics viscosity.

$$
y^{+}=\frac{u_{*} y}{\nu}
$$

Similar to Reynolds Number, $y+$ indicates what part of the boundary layer is being measured. The boundary layer is broken into several layers that are approximated more accurately with using either inner or outer equations [30]. These different layers are separated by $y+$ values. The viscous sublayer ranges from $0.1<y+\lesssim 5$, the buffer layer ranges from $5 \lesssim y+<30$, and the log-law inner region spans from $30<$ $y+<350$ [30]. The viscous sublayer and log-law regions have known approximations, whereas the buffer layer is more difficult, so it is better practice to either have a $y+$ in the sublayer or log-law region.

For the realizable $k-\epsilon$ two layer turbulence model, the recommended $y+$ value 
is $y+\sim 1$ for lower Reynolds numbers [28]. The prism layers were sized to meet this criteria.

\subsubsection{Prism Layers}

Prism layers are high aspect ratio, rectangular prism cells that are meshed on the surfaces and boundaries. They were only needed on no-slip boundaries and were used to capture the boundary layer. The boundary layer contains a large flow field gradient within a small distance, and the high aspect ratio cells were used to capture the transition.

The sizing methodology of the prism layers were broken into two parts: on the surfboard surface and on the fin itself. For the fin, it was important to meet the appropriate $y+\sim 1$ to capture and compare shear interactions across it. The prism layers were sized so that the first prism layer met this value and that the total prism layer height captured the estimated boundary layer thickness. The final boundary layer thickness was approximated using the Blasius solution for a flat plate, Equation 5.3.

$$
\delta=\frac{5.0 x}{\sqrt{R e}}
$$

The Blasius solution is derived from an infinitely long flat plate, so it does not incorporate curvature and is an estimation. It is useful for an approximate boundary layer thickness which will be used as the total prism layer height. Using Equation 5.3 with $x=M H C=0.745 \mathrm{~m}$ and $R e_{x}=3.51 \times 10^{5}$, the boundary layer thickness was calculated as $\delta=0.0271 \mathrm{~m}$.

The next step was to calculate the desired first cell height and was done again using flat plate boundary layer theory [30]. First the friction coefficient was found, $C_{f}$, and then the wall shear stress, $\tau_{\text {wall }}$.

$$
C_{f}=\frac{0.026}{R E^{\frac{1}{7}}}
$$




$$
\tau_{\text {wall }}=\frac{C_{f} \rho U_{\infty}^{2}}{2}
$$

The final steps were to calculate $u_{*}$ and then to re-arrange Equation 5.2 to solve for $y$.

$$
\begin{gathered}
u_{*}=\sqrt{\frac{\tau_{\text {wall }}}{\rho}} \\
y=\frac{u_{*} y^{+}}{\nu}
\end{gathered}
$$

In doing so, the first layer prism height is found to be $y=4.71 \times 10^{-5}$, and the prism layer cells were defined by this height and estimated boundary layer thickness. An iterative process was used to determine the number of layers used, summarized in table Table 5.4. It should be noted that the first layer height in the final iteration was

Table 5.4: Iterations of prism layer sizing on the surfboard fin. Iteration First Layer Height Total Thickness (m) Number of Layers

\begin{tabular}{llll}
\hline 1 & $4.71 \mathrm{E}-05$ & 0.0271 & 20 \\
\hline 2 & $4.71 \mathrm{E}-05$ & 0.0271 & 25 \\
\hline 3 & $9.42 \mathrm{E}-05$ & 0.0271 & 25 \\
\hline
\end{tabular}

increased to double the previous value. This was done because the first two iterations had $y+$ values too small, and after doing so, the appropriate scaling was reached as seen in Figure 5.4. The majority of the fin surface area is in the range close to 1.0 and was deemed satisfactory for accurate convergence.

After the prism layer sizing of the fin, the prism layers were set for the no-slip surface on the surfboard bottom. This followed a similar methodology. The boundary layer thickness was recalculated using the length of the no-slip surface, $17.7 \mathrm{~m}$ and the Reynolds number of the surface, $8.34 \times 10^{6}$ and was found to be $\delta=0.270 \mathrm{~m}$. The $y+$ value for this surface was less critical to match. It was more important to capture the turbulent boundary layer than it was to calculate accurate shear stress values, as 


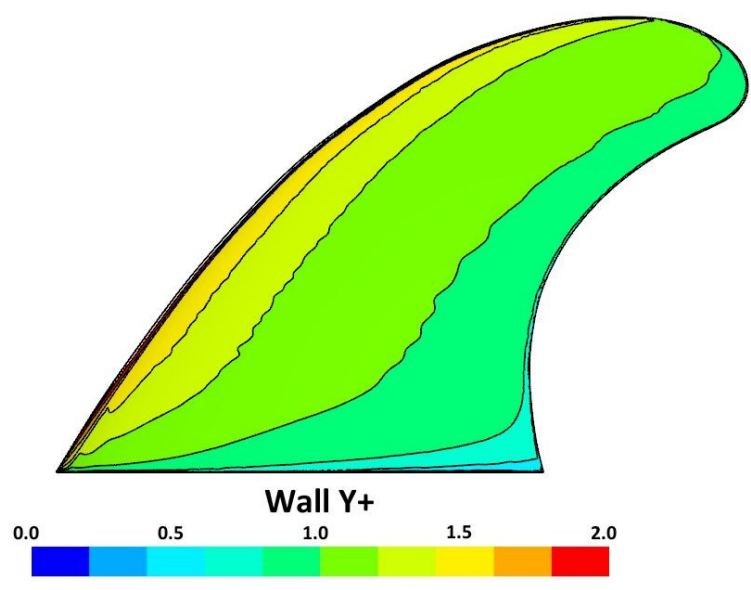

Figure 5.4: Wall $y+$ for the baseline geometry at $\alpha_{F}=0^{\circ}$.

the fin was the body being measured. In order to meet a desired low $y+$ value, the number of prism layers has to increase close to the surface. This can sometimes lead to an added cell count of million or more, so to save cells, less prism layers were used on the surfboard surface. The final total thickness of the prism layers was $0.15 \mathrm{~m}$,

Table 5.5: Iterations of prism layer sizing on the surfboard no-slip surface.

\begin{tabular}{cccc}
\hline Iteration & First Layer Height $(\mathbf{m})$ & Total Thickness $(\mathbf{m})$ & Number of Layers \\
\hline 1 & 0.001 & 0.273 & 15 \\
\hline 2 & 0.001 & 0.200 & 10 \\
\hline 3 & 0.001 & 0.150 & 10 \\
\hline
\end{tabular}

which was less than the predicted boundary layer thickness. This was because after this height the prism layer cells began to blend in properly with the volume mesh off the surface.

\subsubsection{Mesh Independence}

The mesh sizing focused on three different areas: domain cell size, wake cell size, and fin surface size. Similar to the domain sizing, each of these three areas had their mesh settings varied until there was no longer a noticeable change in forces on the 
fin. There were a total of nine iterations tested. The detail of each setting used is tabulated in Appendix A in Table A.2 through Table A.4.

Figure 5.5 displays the results of the mesh independence study, with the $x$ axis as the cell count and the $y$ axis split into two, the fin lift and drag coefficients. The numbers labeling the points correspond to the iteration.

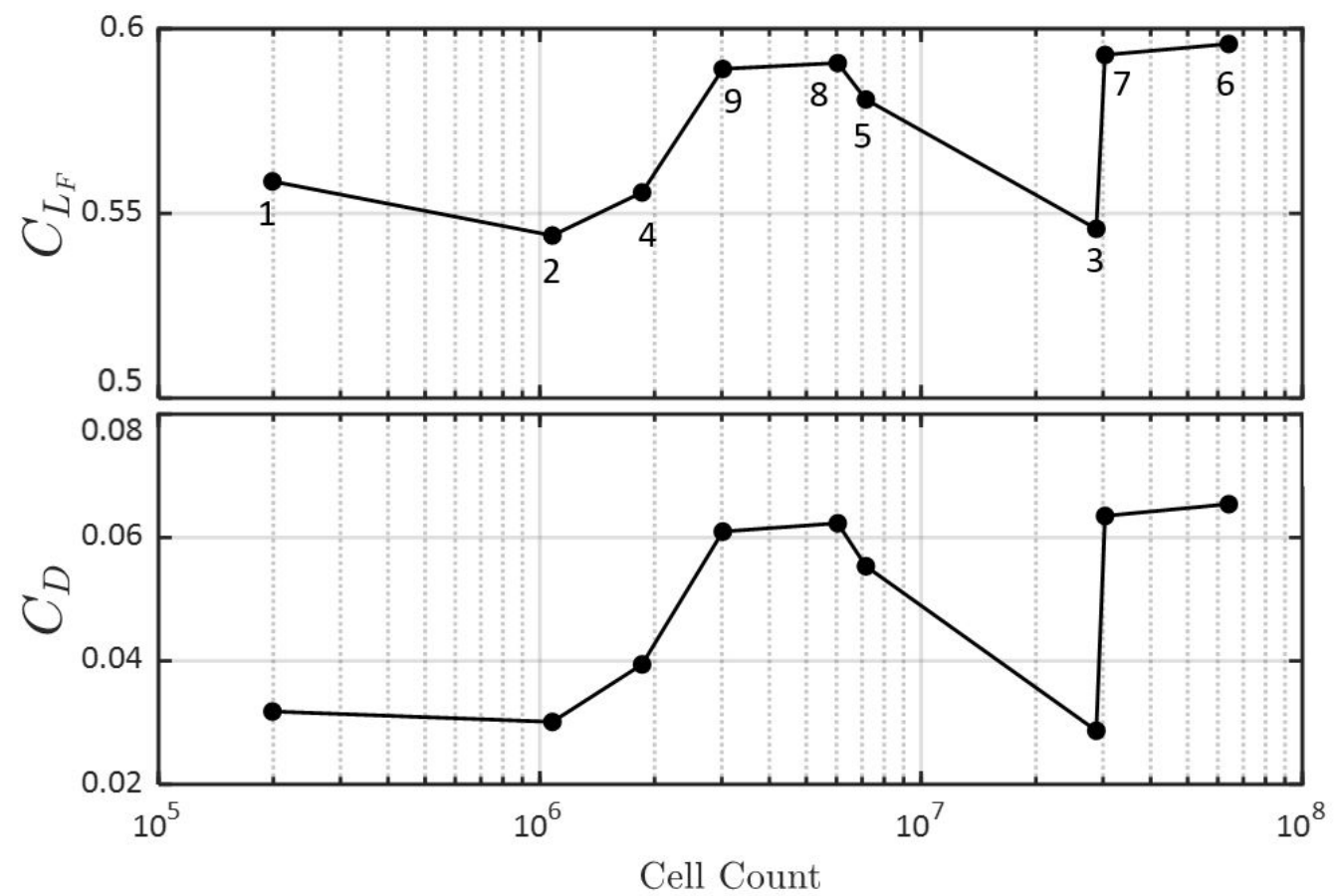

Figure 5.5: Results of mesh sizing study. The numbers correspond to the iteration.

After $1 \times 10^{6}$ cells, the results of each mesh size begin to plateau for both the fin lift and drag coefficient with a single outliers at a cell count of $2.88 \times 10^{7}$. This was the third iteration, and because this was an earlier iteration the wake and surface size had not yet been sized properly, so the values generated were closer to the lower cell count cases.

The final mesh settings came from the eighth iteration, which was the $5.0 \times 10^{6}$ cell case. This was an order of magnitude less cells than the highest cell count and had a percent difference less than $1.0 \%$ for $C_{L_{F}}$ and $C_{D}$. These settings would be 
used for the rest of the imported geometries, and a summary can be seen in Table 5.6.

Table 5.6: Summary of final mesh settings. The wake length refers to the distance of the wake refinement aft of the fin, and the wake density is the target cell size within the wake refinement.

\begin{tabular}{ccc}
\hline Domain Size (m) & Growth Rate & Fin Surface Size (m) \\
\hline 0.50 & 1.00 & 0.0175 \\
\hline Wake Length $(\mathbf{m})$ & Wake Angle & Wake Density (m) \\
\hline 3.00 & $20^{\circ}$ & 0.040 \\
\hline
\end{tabular}

\subsection{Validation Test Case}

An attempt was made to validate the mesh settings and domain size using a comparison simulation against the study completed by Brandner and Walker in Section 1.6 [4]. The same geometry was used from their water tunnel test but simulated with the mesh settings and domain size found in the previous section. It is important to note that a point of difference in doing so is that the domain did not imitate the wall effects of their water tunnel or the boundary layer growth of the test section. After these simulations were run, it was also discovered that the geometry was not a perfect match to the original geometry.

A Reynolds number of $4.0 \times 10^{5}$ was matched, and the CFD simulations were run at the same $\alpha_{F}=0^{\circ}: 4^{\circ}: 24^{\circ}$. The body forces between the two studies were than compared.

Figure 5.6, Figure 5.7 and Figure 5.8 show the results of this comparison. One of the noticeable differences is the CFD simulations predict $C_{L_{F}, \max }$ much earlier than the Brandner and Walker study. Brandner and Walker's water tunnel tests found that their fin reached $C_{L_{F}, \max }$ at $\alpha_{F}=24^{\circ}$, whereas the validation CFD predicts $C_{L_{F}, \max }$ at $\alpha_{F}=16^{\circ}$. 


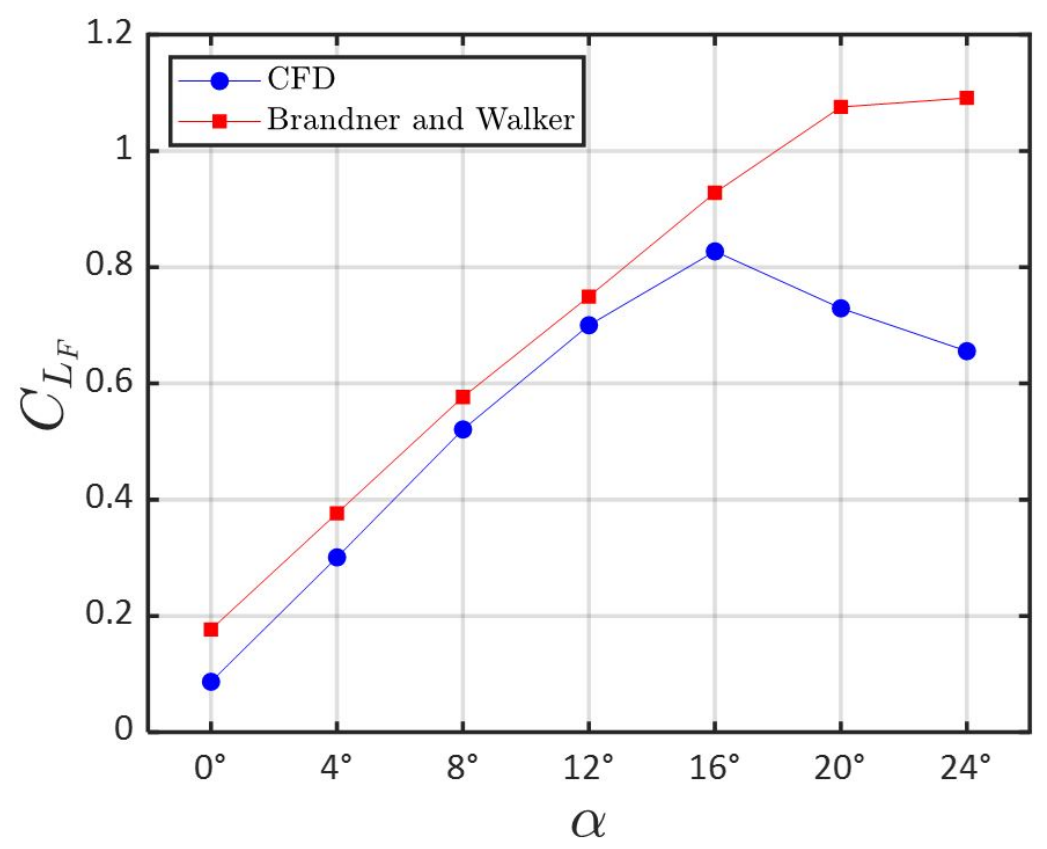

Figure 5.6: Comparison of $C_{L_{F}}$ for the Brandner and Walker test [4] and the Validation CFD at $R e=4.0 \times 10^{5}$

$C_{M}$ and $C_{D}$ were not as close to the water tunnel tests. Both show similarly trending values, in that both trend down and up respectively with an increase in $\alpha_{F}$, up until $\alpha_{F}=16^{\circ}$, but the slopes differ from the Brandner and Walker study. Both $C_{M}$ and $C_{D}$ increase in magnitude faster. While these two plots are not as similar to $C_{L_{F}}$, both follow the same trend and are within the same order of magnitude, giving confidence to move forward. 


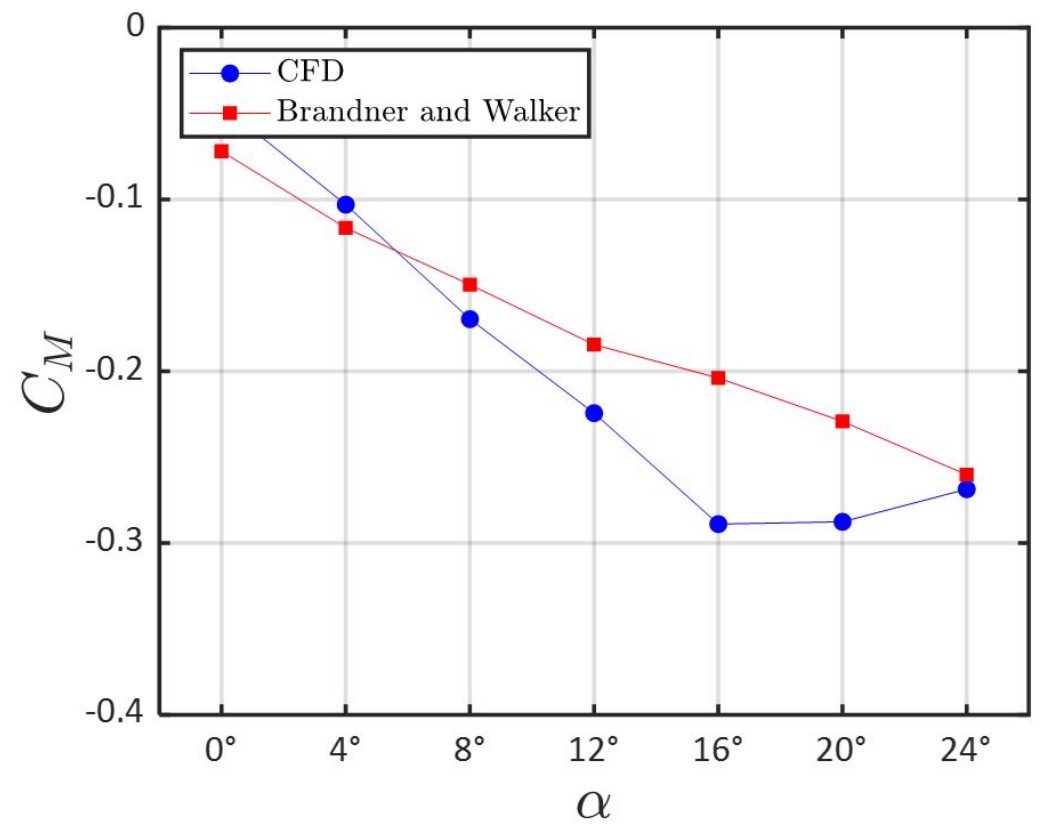

Figure 5.7: Comparison of $C_{M}$ for the Brandner and Walker test [4] and the Validation CFD at $R e=4.0 \times 10^{5}$

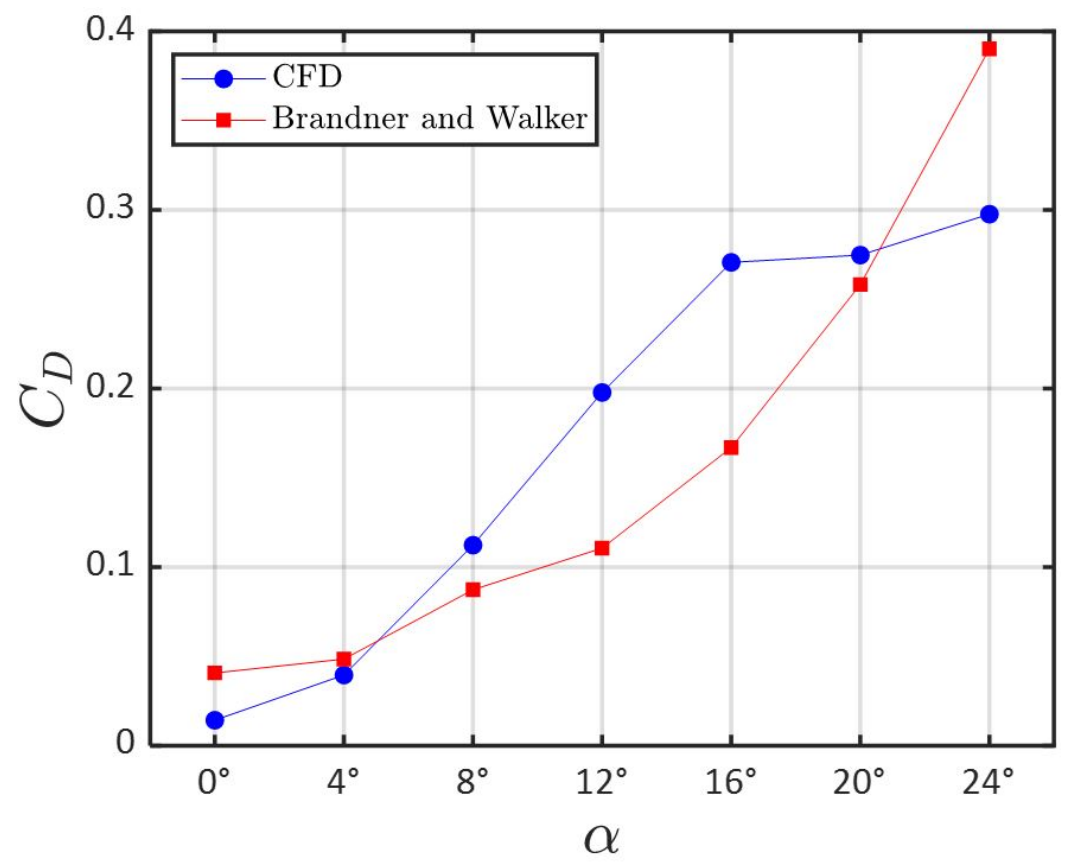

Figure 5.8: Comparison of $C_{D}$ for the Brandner and Walker test [4] and the Validation CFD at $R e=4.0 \times 10^{5}$ 
Chapter 6

RESULTS AND ANALYSIS

Each of the fin geometries was simulated at a $R e=3.51 \times 10^{5}$ and $\alpha_{F}=0^{\circ}, 5^{\circ}, 10^{\circ}$ : $20^{\circ}$. The following results begin with an analysis of the baseline geometry, followed with a comparison of the altered geometry sets.

\subsection{Baseline}

For the baseline geometry, the body forces were measured and reported along with visual cross sections of skin friction and vorticity at each incidence angle.

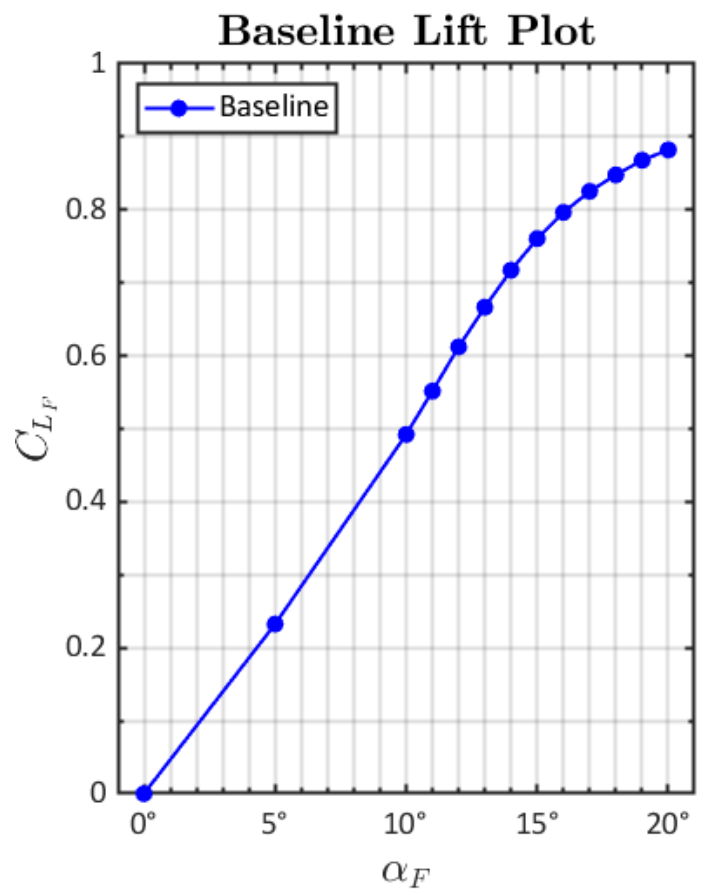

Figure 6.1: Lift plot for the baseline fin configuration

Figure 6.1 shows the change in $C_{L_{F}}$ with respect to $\alpha_{F}$. It should be noted that for this figure and for the following plots, error bars were not included, as they were 
less than $1 \%$ of the resultant values and overcrowded the plots. The lift curve slope, $\alpha_{0}$, was measured to be $\alpha_{0}=0.0492$, and through $\alpha_{F}=20^{\circ}$, there was still no drop in $C_{L_{F}}$, implying that the maximum $C_{L_{F}}$ value had not been reached. To investigate this, the lift distribution was analyzed at each incidence angle.

The lift distribution along the fin's span was measured and shown in Figure 6.2. This was done by using $C_{p}$ distributions at $0.5 \mathrm{~m}$ increments along the span of the fin. The data was then input into a panel code. The code broke the pressure force into both a normal and axial component relative to the surface of the hydrofoil and freestream direction. The normal and axial components were then integrated along the span of the fin to get a two dimensional lift and drag coefficient $\left(c_{l_{F}}\right.$ and $\left.c_{d}\right)$ for each cross section. When plotted with respect to the span of the fin, the distribution of lift along the fin surface is depicted. The result of this can be seen in Figure 6.2.

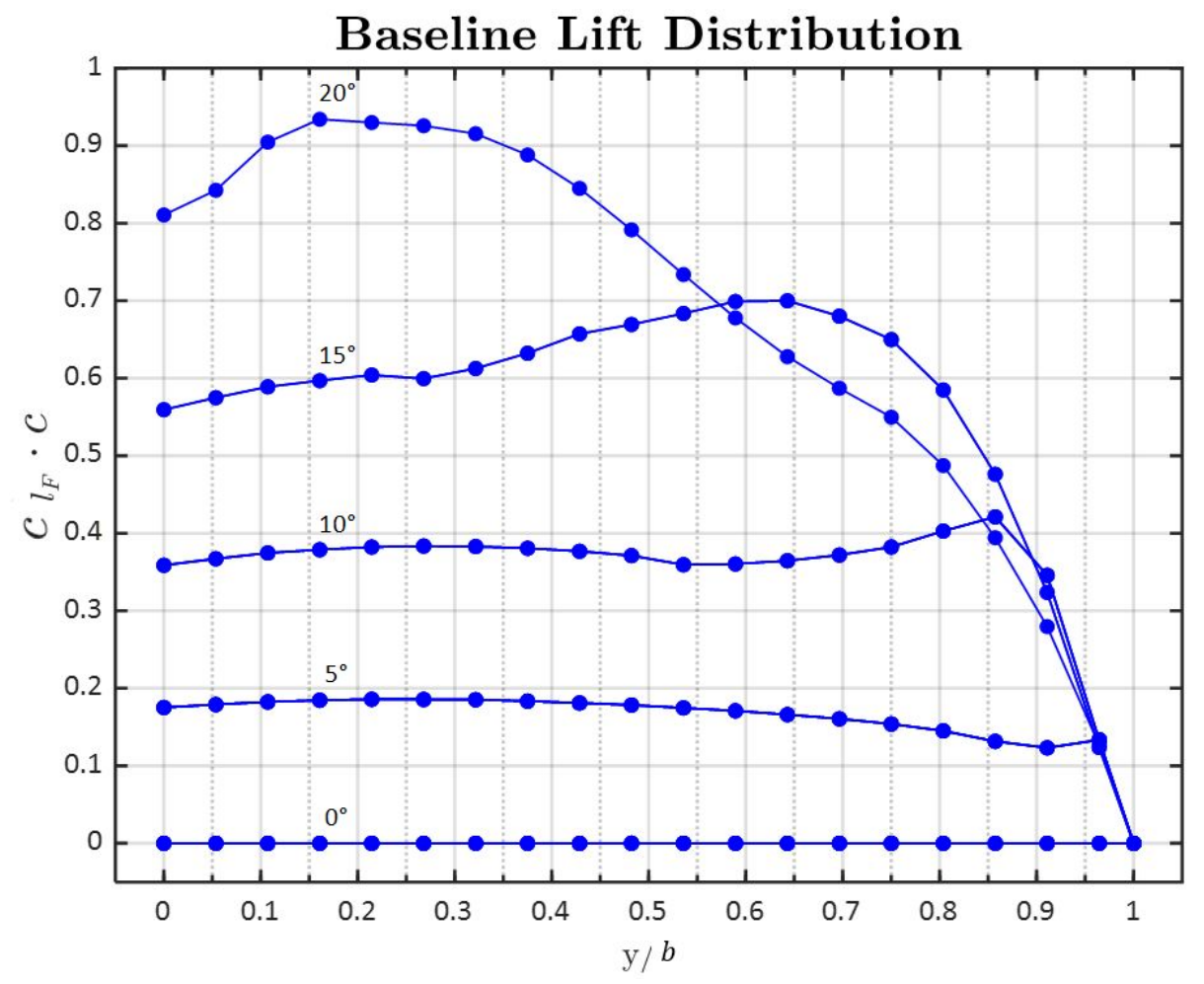

Figure 6.2: Lift distribution for the baseline fin configuration. 
Five lines were plotted in increments of $\alpha_{F}=5^{\circ}$, and $c_{l_{F}}$ was multiplied by the local chord length to weight the amount of lift produced at a given location. At $\alpha_{F}=0^{\circ}$ and $\alpha_{F}=5^{\circ}$, the lift distribution remains relatively flat, but at $\alpha_{F}=10^{\circ}$, there is a noticeable rise in lift close to the fin tip. This was the first indication that there was a low pressure zone generated from either a vortex or separation. For this case, it was the beginning of a conical vortex.

This is highlighted in Figure 6.3, which shows skin friction coefficient contours along the suction surface of the fin. The skin friction coefficient is defined by Equation 6.1 , where $\tau_{W}$ is the local wall shear stress, $\rho$ is density and $U_{\infty}$ is the freestream

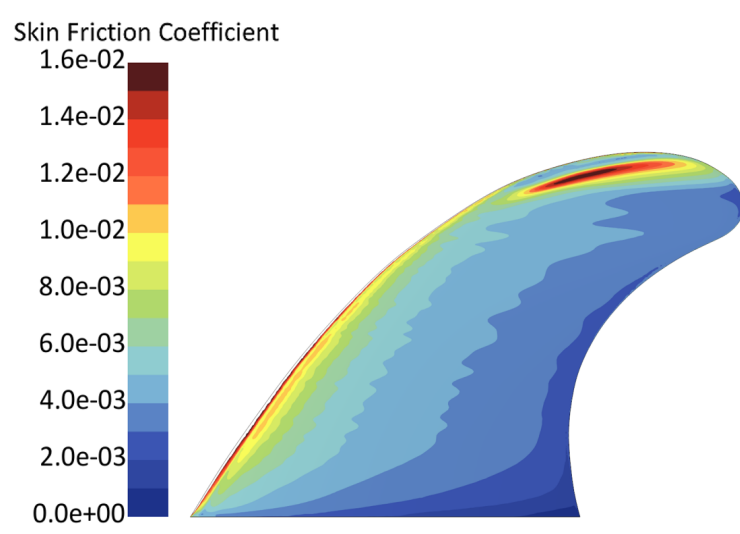

(a) $\alpha_{F}=5^{\circ}$

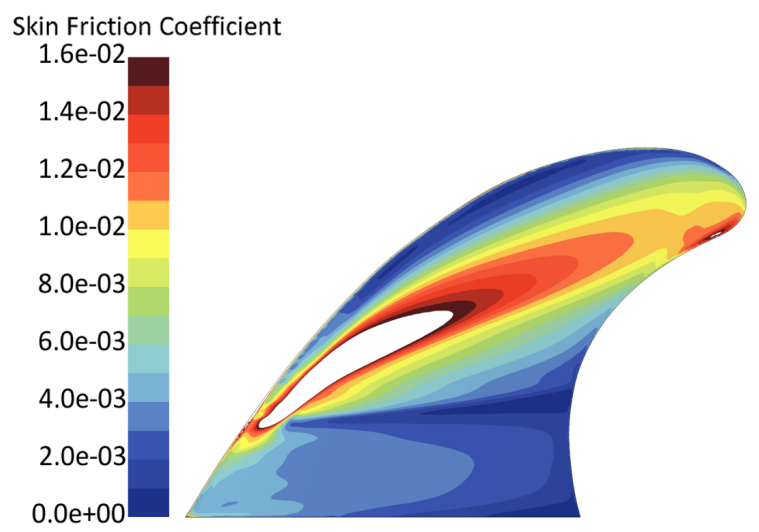

(c) $\alpha_{F}=15^{\circ}$

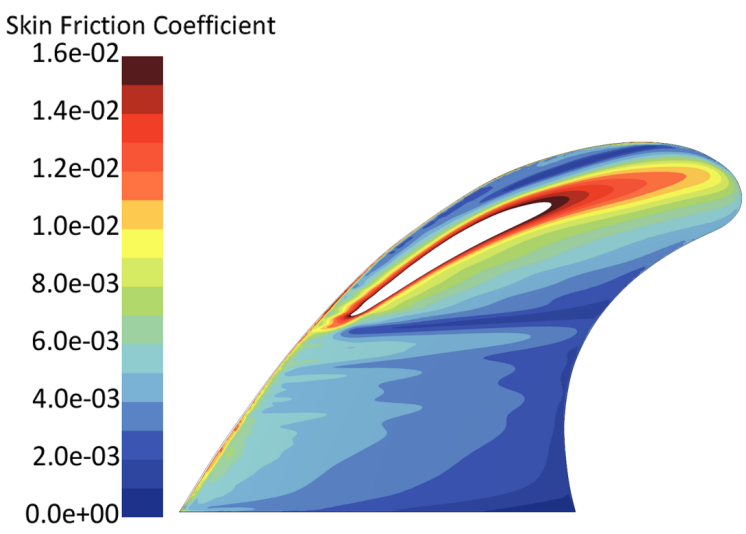

(b) $\alpha_{F}=10^{\circ}$

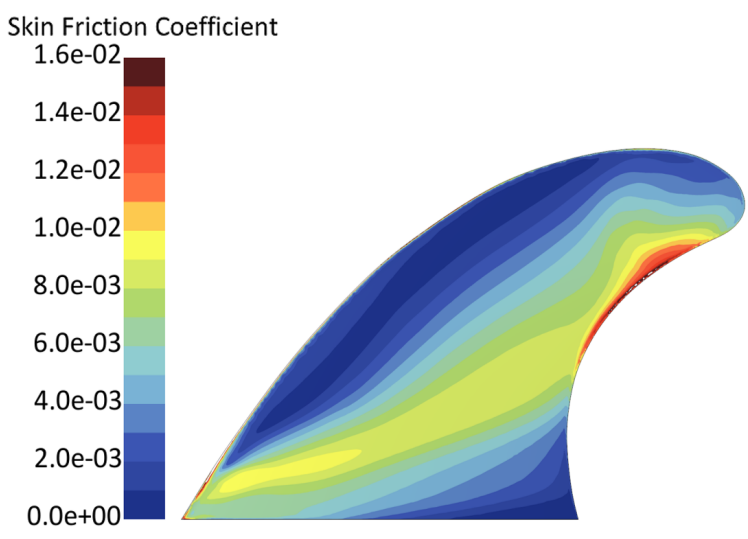

(d) $\alpha_{F}=20^{\circ}$

Figure 6.3: Skin friction coefficient comparison for the baseline fin configuration. 
velocity. It is a non-dimensional form of expressing the amount of shear stress on the surface of an object.

$$
C_{f}=\frac{\tau_{w}}{\frac{1}{2} \rho U_{\infty}^{2}}
$$

Any areas of white on the fin surface of Figure 6.3 indicate an area where the skin friction coefficient exceeded the maximum value of the legend $\left(C_{f}=1.6 \times 10^{-2}\right)$. At $\alpha_{F}=5^{\circ}$ (Figure 6.3a), there was a high shear streak of $C_{f}=1.6 \times 10^{-2}$ towards the fin tip. This area grows and moves towards the root chord as the angle increases and was indicative that the conical vortex began its formation closer to the root chord. This was why the larger lift coefficients in Figure 6.2 moved from the fin tip to the

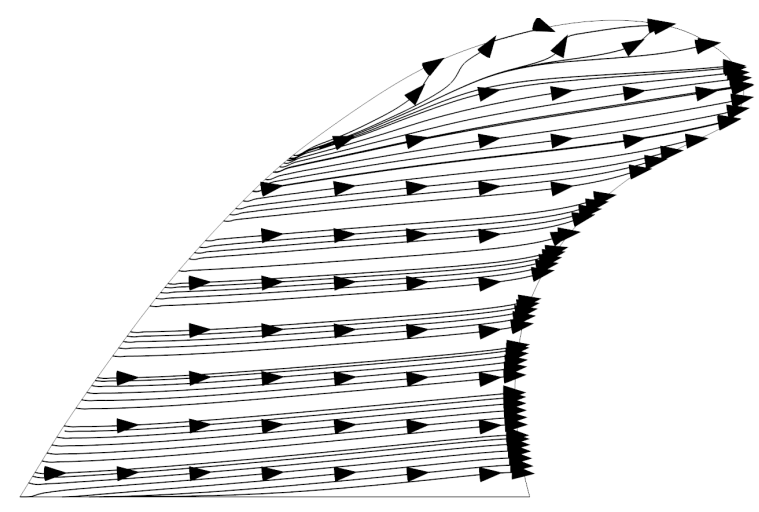

(a) $\alpha_{F}=5^{\circ}$

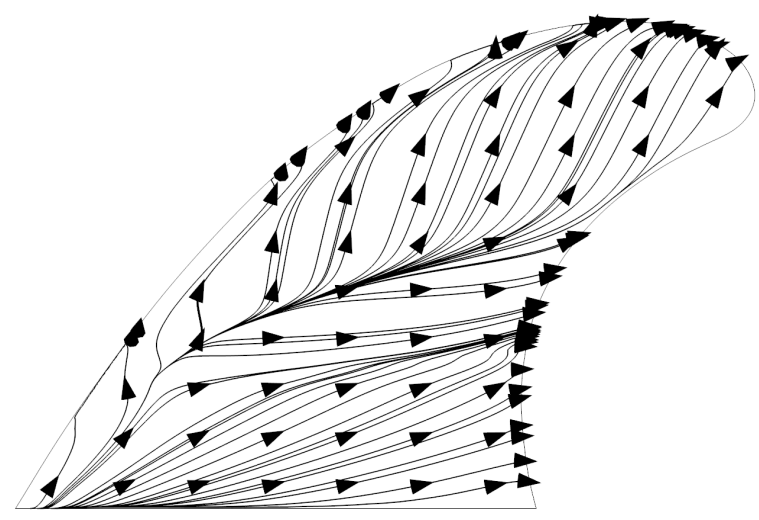

(c) $\alpha_{F}=15^{\circ}$

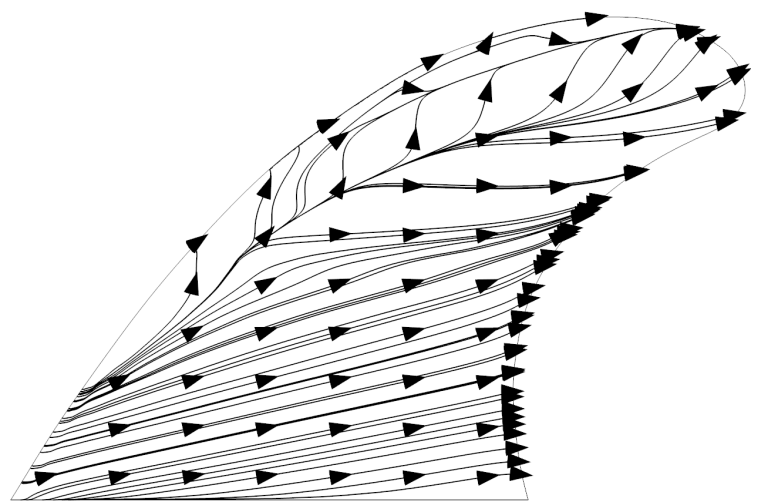

(b) $\alpha_{F}=10^{\circ}$

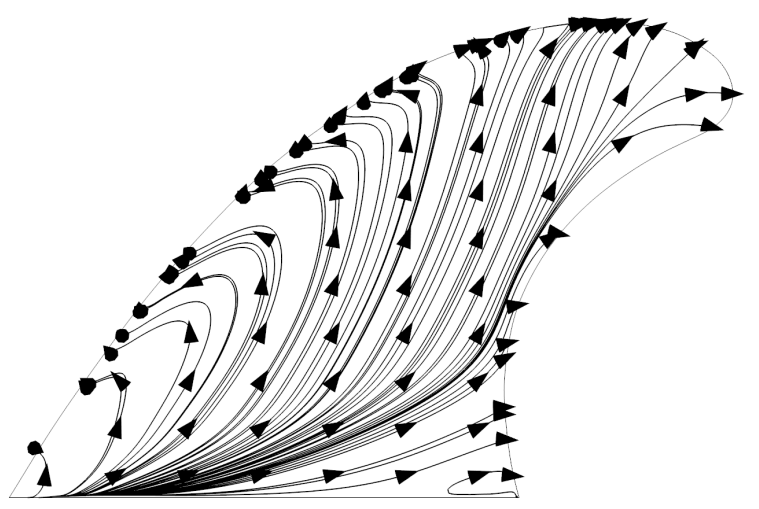

(d) $\alpha_{F}=20^{\circ}$

Figure 6.4: Streakline comparison for the baseline fin configuration. 
root chord.

At $\alpha_{F}=20^{\circ}$, the lift distribution, max value was even closer to the root chord at $\frac{y}{b}=0.2$, but there were no longer shear areas above $C_{f}=8.0 \times 10^{-3}$. Instead, as highlighted in Figure 6.4, there was an area of recirculation.

Figure 6.4 shows streaklines along the suction surface of the fin. At $\alpha_{F}=5^{\circ}, 10^{\circ}$ and $15^{\circ}$, the streaklines swirl and intertwine through the fin tip, showing the rotation of a vortex. However, at $\alpha_{F}=20^{\circ}$, there are no longer any intertwining lines but an elliptical pattern.

This showed and indicated three different flow patterns around the fin. Starting with either zero or low incidence to the freestream flow, there were no notable flow features. As the incidence angle increased, a vortex began to roll up along the leading edge and create a low pressure, high shear region on the suction surface. Eventually, the structure of the vortex was lost and replaced by a recirculating, separated region. This meant there was a constant low pressure region on the suction surface of the fin and was a similar phenomena to swept and delta wing geometry.

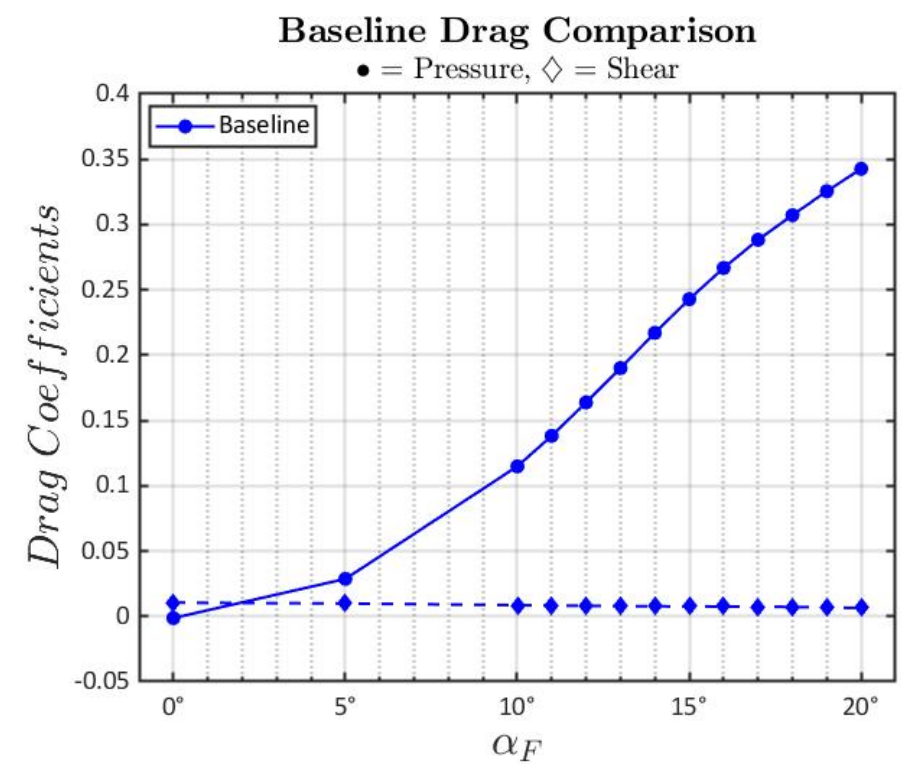

Figure 6.5: Pressure versus shear drag for the baseline fin. 
Figure 6.5 shows both the shear and pressure components of drag with increasing incidence angle. Because the switch from a structured vortex to separation is gradual, the increase in pressure drag remains relatively constant through the higher angles.

The switch from vortex to separation can be visualized in Figure 6.6, which shows vorticity contours at $0.5 \mathrm{~m}$ increments along the fin. At $\alpha_{F}=10^{\circ}$, the vortex core formed off the leading edge, and even though the strength dissipates, the structure of the vortex persisted through the fin tip. However, starting at $\alpha_{F}=15^{\circ}$, a vortex core was generated but quickly turns into a large re-circulating area. At $\alpha_{F}=20^{\circ}$, there was no longer a vortex core. This was the dominant flow feature seen on the baseline geometry and was used as a point of comparison to the other fin geometries. 


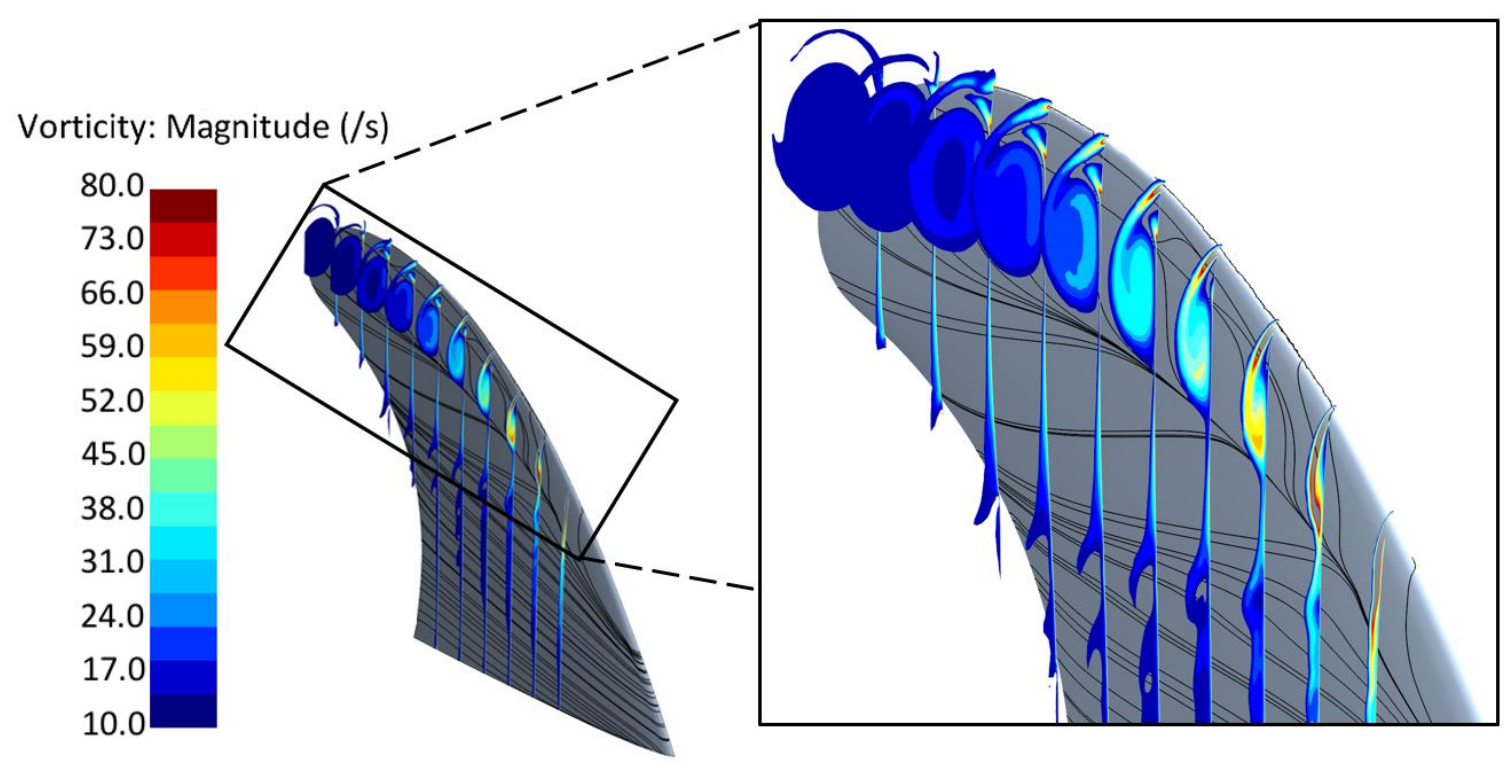

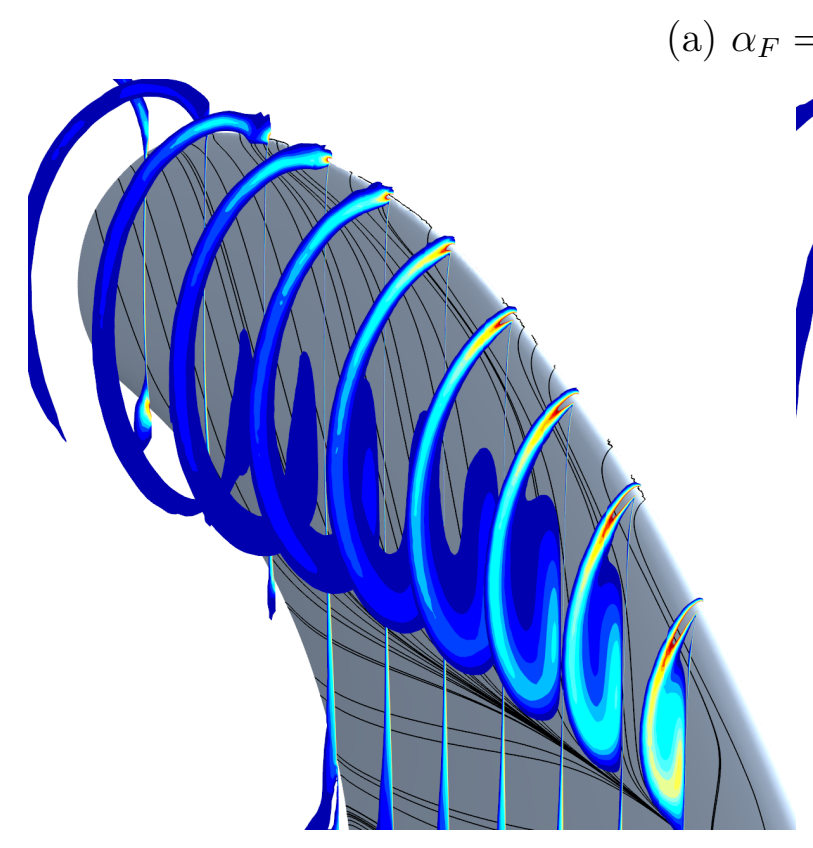

(b) $\alpha_{F}=15^{\circ}$

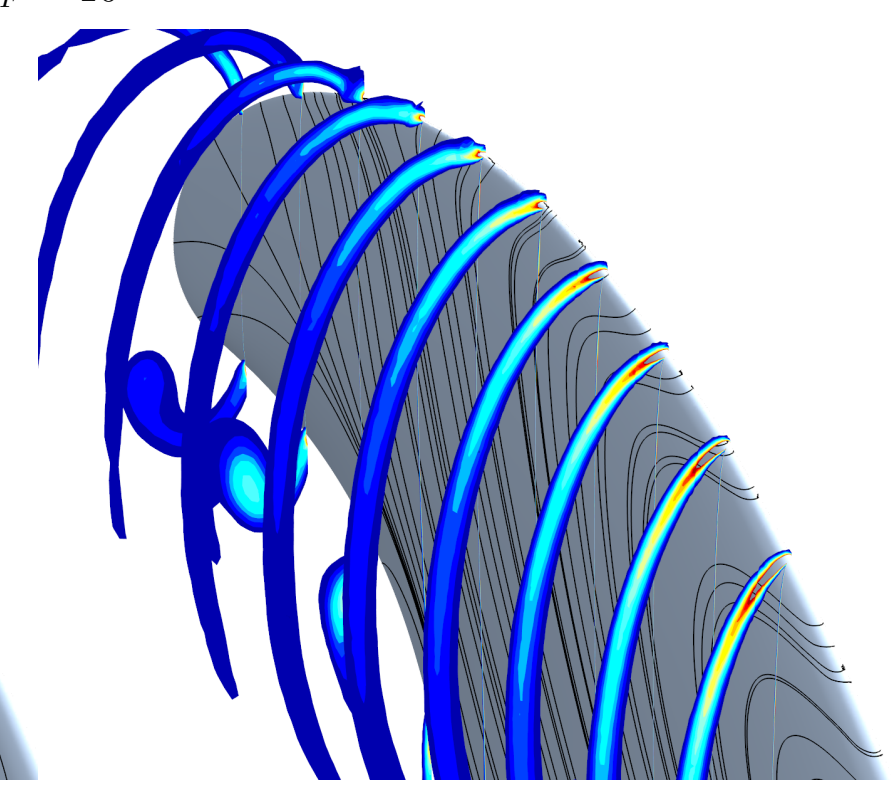

(c) $\alpha_{F}=20^{\circ}$

Figure 6.6: Vorticity comparison for the baseline fin configuration. Each vorticity cross section is at $0.1 \mathrm{~m}$, and the fin surface has streakline visualization. 


\subsection{Sweep}

There were a total of eight different swept fin geometries $\left(\Lambda_{\text {tip }}=15^{\circ}, 20^{\circ}, 25^{\circ}, 30^{\circ}, 40^{\circ}\right.$, $45^{\circ}, 50^{\circ}$ and $\left.55^{\circ}\right)$ simulated and compared to the baseline geometry $\left(\Lambda_{\text {tip }}=35^{\circ}\right)$. Each of these geometries had the same taper distributions, but the fins' sweep distribution was proportionally varied. The shapes were simulated at the same conditions as the baseline geometry: $R e=3.51 \times 10^{5}$ and $\alpha_{F}=0^{\circ}, 5^{\circ}, 10^{\circ}: 20^{\circ}$.

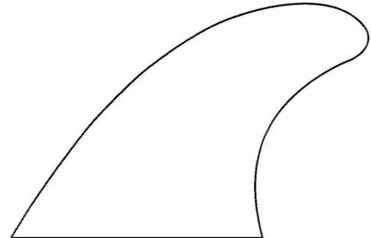

(a) $\Lambda_{\text {tip }}=35^{\circ}$ (Baseline)

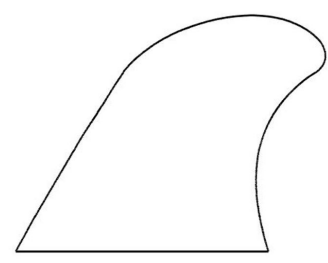

(d) $\Lambda_{t i p}=25^{\circ}$

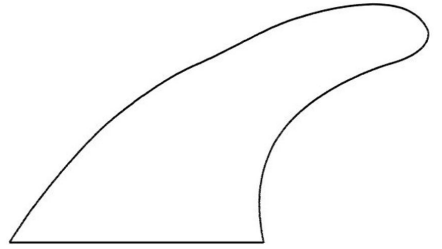

(g) $\Lambda_{\text {tip }}=45^{\circ}$

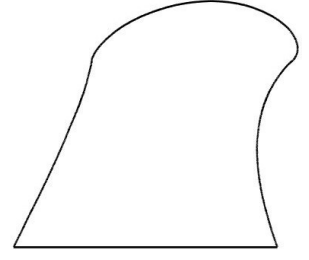

(b) $\Lambda_{\text {tip }}=15^{\circ}$

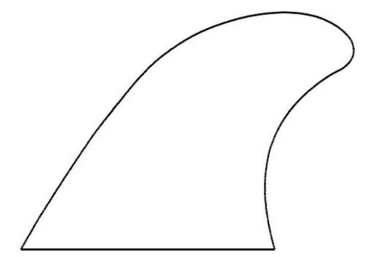

(e) $\Lambda_{t i p}=30^{\circ}$

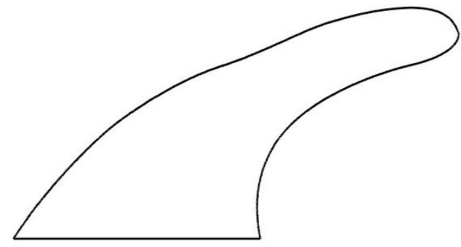

(h) $\Lambda_{t i p}=50^{\circ}$

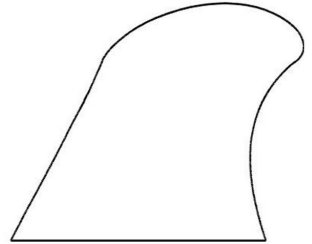

(c) $\Lambda_{\text {tip }}=20^{\circ}$

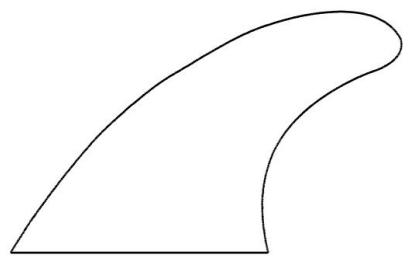

(f) $\Lambda_{\text {tip }}=40^{\circ}$

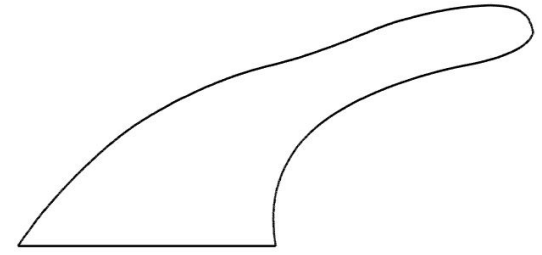

(i) $\Lambda_{\text {tip }}=55^{\circ}$

Figure 6.7: Planform shapes of swept geometries.

\subsubsection{Sweep Geometry}

Figure 6.7 shows the results of the geometry creation using new proportionally swept values. Each fin geometry will be referred to be its final tip sweep value, $\Lambda_{t i p}$, and the fins with $\Lambda_{t i p}$ values of $15^{\circ}$ and $55^{\circ}$ were minimum and maximum differences in 
sweep. Appendix B.2 shows more detail on each geometry.

\subsection{2 $\Lambda_{t i p}=15^{\circ}, 20^{\circ}, 25^{\circ}$ and $30^{\circ}$ Lift, Shear and Vorticity}

The lower $\Lambda_{t i p}\left(\Lambda_{t i p}=15^{\circ}, 20^{\circ}, 25^{\circ}, 30^{\circ}\right)$ fin lift curves are shown in Figure 6.8. Previously, it was difficult to discern where and if the baseline fin geometry saw separation, as there was no drop off in $C_{L_{F}}$, but Figure 6.8 shows that each of the less swept fins have distinct points after a given critical $\alpha_{F}$ where $C_{L_{F}}$ decreases. $\Lambda_{t i p}=15^{\circ}$ had the smallest value of $C_{L_{F}, \max }=0.61$, and as $\Lambda_{t i p}$ increases, $C_{L_{F}, \max }$ increases as well along the angle at which it occurs at.

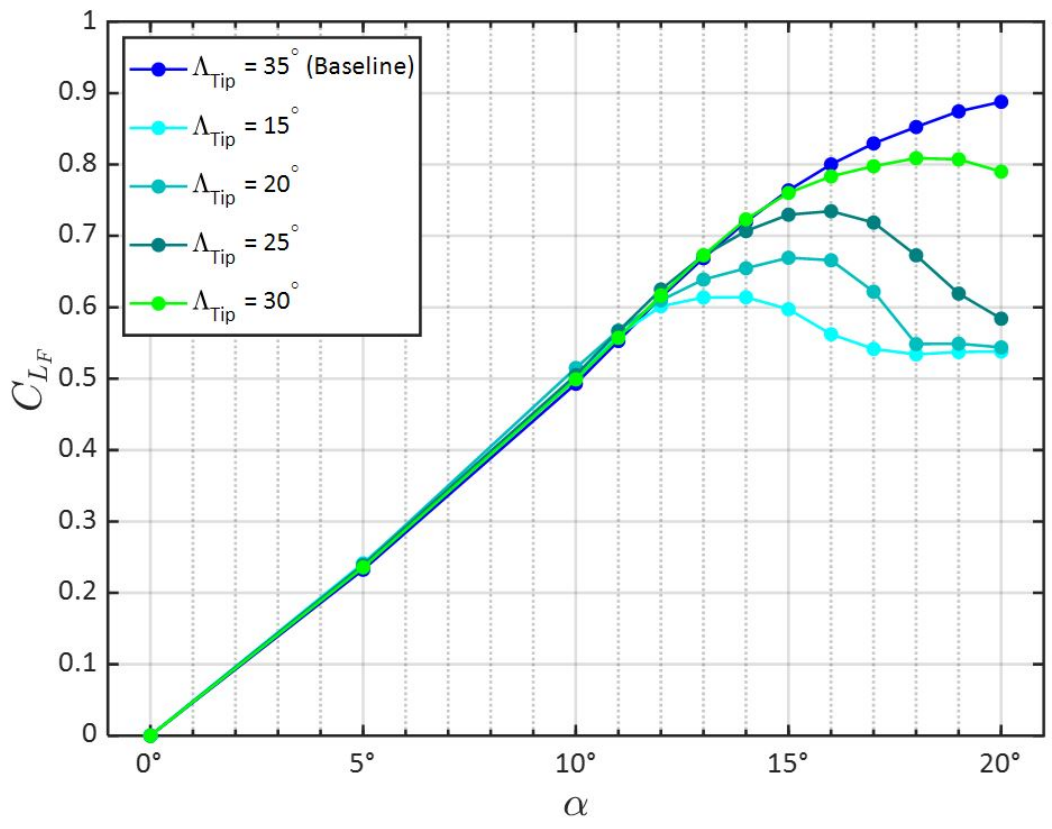

Figure 6.8: Lift curve comparison for $\Lambda_{t i p}=15^{\circ}, 20^{\circ}, 25^{\circ}$, and $30^{\circ}$.

Each lift curve also had similar lift curve slopes; the maximum slope was 0.0507 for $\Lambda_{t i p}=20^{\circ}$ and a minimum of 0.0498 for $\Lambda_{t i p}=30^{\circ}$, giving a maximum percent difference from the baseline slope of $2.98 \%$. To investigate the difference in $C_{L_{F}, \max }$, the skin friction coefficient was analyzed.

Figure 6.9 shows skin friction coefficient contours on the suction surface at $\alpha_{F}=$ 


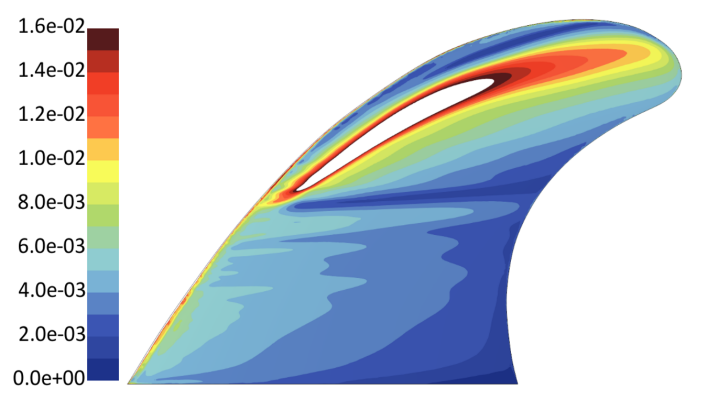

(a) $\Lambda_{t i p}=35^{\circ}$ (baseline)

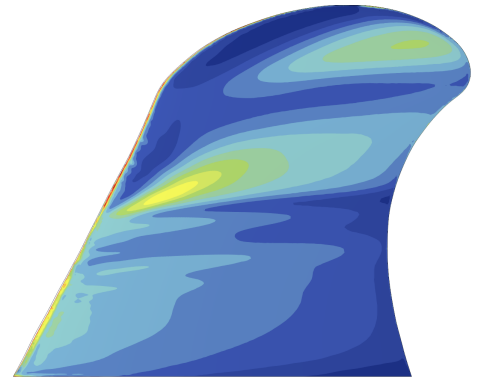

(c) $\Lambda_{t i p}=20^{\circ}$

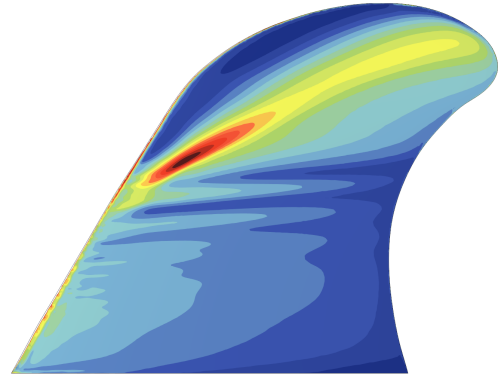

(d) $\Lambda_{t i p}=25^{\circ}$

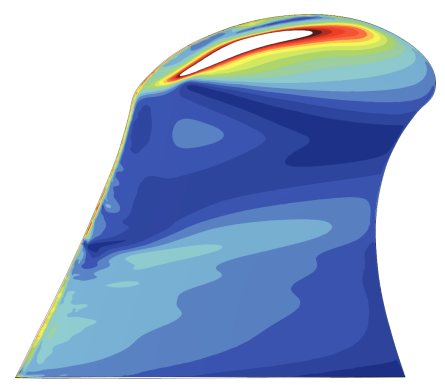

(b) $\Lambda_{\text {tip }}=15^{\circ}$

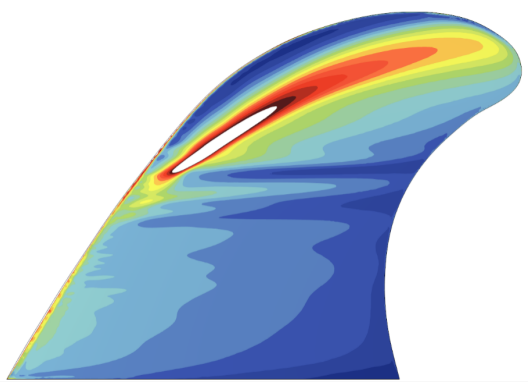

(e) $\Lambda_{t i p}=30^{\circ}$

Figure 6.9: $\alpha_{F}=10^{\circ}$, skin friction coefficient comparison on the low pressure surface for the fins with decreasingly less sweep. Areas of white on the fin surface indicate high shear areas that exceed the maximum scalar value.

$10^{\circ}$. Again, any areas on the surface that were white exceeded the maximum bound of the scalar legend, indicating areas of high shear stress. When looking at the skin friction coefficient in Figure 6.9, there was a notable difference in flow behavior. Starting with $\Lambda_{t i p}=20^{\circ}$ in Figure 6.9c, there was an area highlighted by light green, halfway up the leading edge where $C_{F}=8.0 \times 10^{-3}$. As the sweep of the different fin geometry increases from $\Lambda_{t i p}=20^{\circ}$ to $\Lambda_{t i p}=30^{\circ}$, this area increased in shear stress and followed a path closer to the leading edge, indicating that increasing the sweep increased the shear stress from vorticity.

The $\Lambda_{t i p}=15^{\circ}$ case in Figure $6.9 \mathrm{~b}$ did not follow this trend, due to the protrusion forward on the leading edge. The $\Lambda_{t i p}=15^{\circ}$ fin was the only geometry where the leading edge changed direction from sweeping aft to moving back forwards, seen near the fin tip. This was an unexpected product of altering the sweep distribution to the 
smallest $\Lambda_{t i p}$, and as a result, the scalar images indicated that the protrusion acted as a vortex generator. It is difficult to say whether or not this is the only reason for this high shear area, but because this shape was the only one with this feature, and the only one with the forward leading edge; it hints that this is the case.

In order to clarify the differences in $C_{L_{F}}$ further, images of the vorticity off the suction surface were exported, seen in Figure 6.10. Whereas previously in the baseline geometry, there was a strong vortex core that traveled along the leading edge off the tip, $\Lambda_{t i p}=15^{\circ}, 20^{\circ}$, and $25^{\circ}$ show no signs of this. The only vorticity seen is in the boundary layer of the fin or regions of high pressure circulating around the leading edge to the low pressure surface. It is also noted that the hypothesized tip vortex can be seen in Figure $6.10 \mathrm{c}$ for the $\Lambda_{t i p}=15^{\circ}$ case, but at $12^{\circ}$, this vortex has already transitioned into a separated region.

There was a clear distinction of flow features from the baseline geometry to the $\Lambda_{t i p}=15^{\circ}, 20^{\circ}$, and $25^{\circ}$ cases. The lower swept fins did not generate either as strong or any conical vortex. Instead of switching from vortex to separation, there was simply separation once a fin moved past it's critical $\alpha_{F}$. This indicated that decreasing the sweep of the fin influenced this result. 

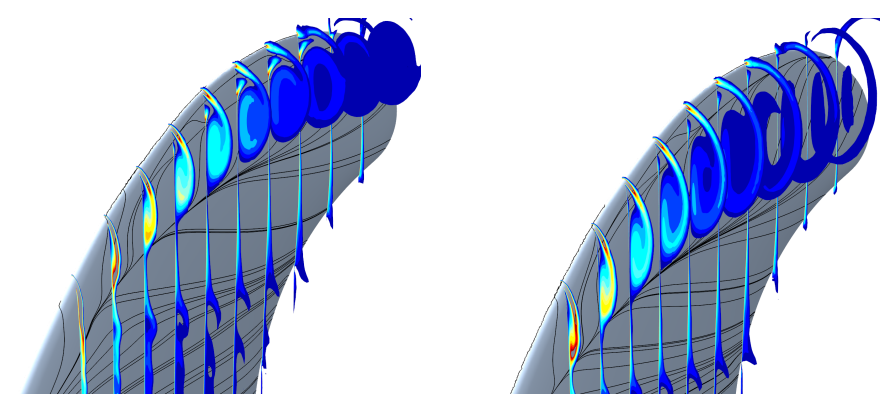

(a) $\Lambda_{\text {tip }}=35^{\circ}$ (baseline),

(b) $\Lambda_{t i p}=35^{\circ}$ (baseline),

$$
\alpha_{F}=10^{\circ}
$$

$$
\alpha_{F}=12^{\circ}
$$
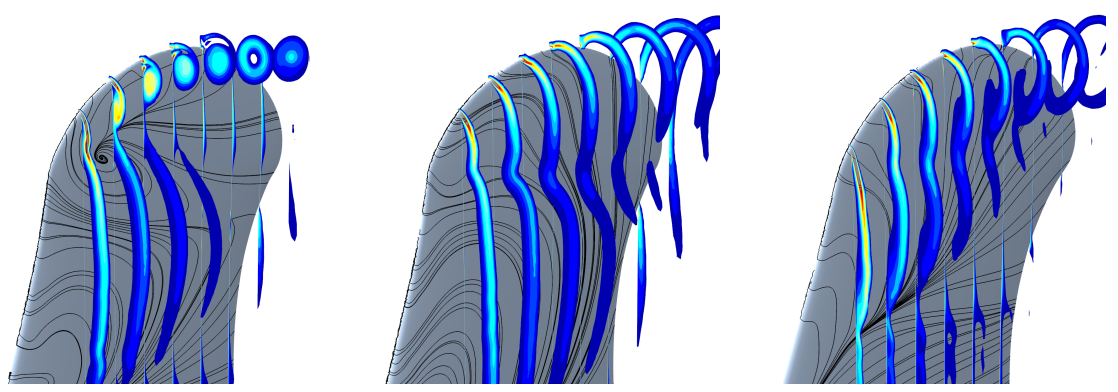

(c) $\Lambda_{t i p}=15^{\circ}$,

(d) $\Lambda_{t i p}=15^{\circ}$,

(e) $\Lambda_{t i p}=20^{\circ}$,

$\alpha_{F}=10^{\circ}$

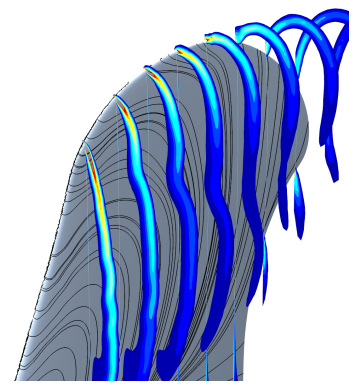

$\alpha_{F}=10^{\circ}$

$\alpha_{F}=12^{\circ}$
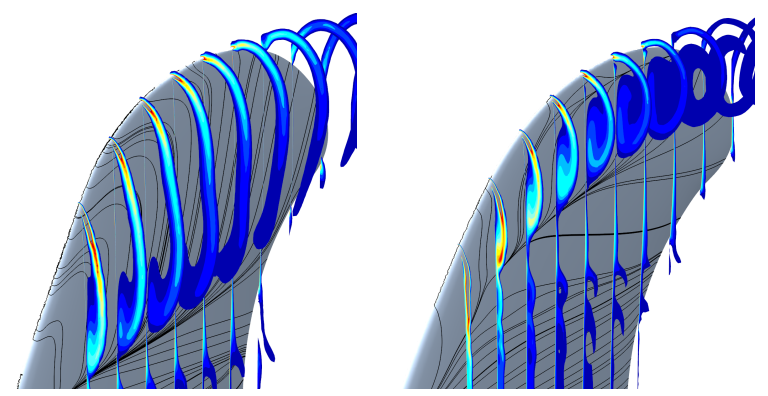

(f) $\Lambda_{t i p}=20^{\circ}$,

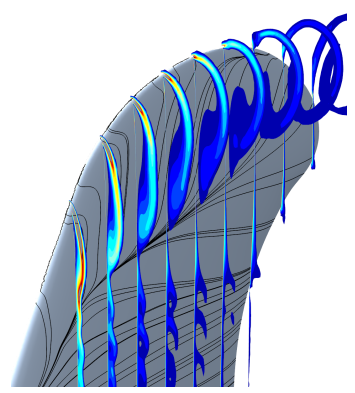

(h) $\Lambda_{\text {tip }}=25^{\circ}$,

(i) $\Lambda_{t i p}=30^{\circ}$,

$\alpha_{F}=12^{\circ}$

(g) $\Lambda_{t i p}=25^{\circ}$,

$\alpha_{F}=12^{\circ}$

$\alpha_{F}=10^{\circ}$

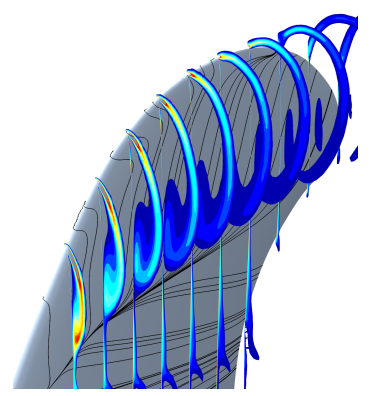

$\alpha_{F}=10^{\circ}$

$\alpha_{F}=10^{\circ}$

(j) $\Lambda_{\text {tip }}=30^{\circ}$,

$$
\alpha_{F}=12^{\circ}
$$

Figure 6.10: Vorticity images for $\Lambda_{t i p}=15^{\circ}$ through $30^{\circ}$ and the baseline geometry. 


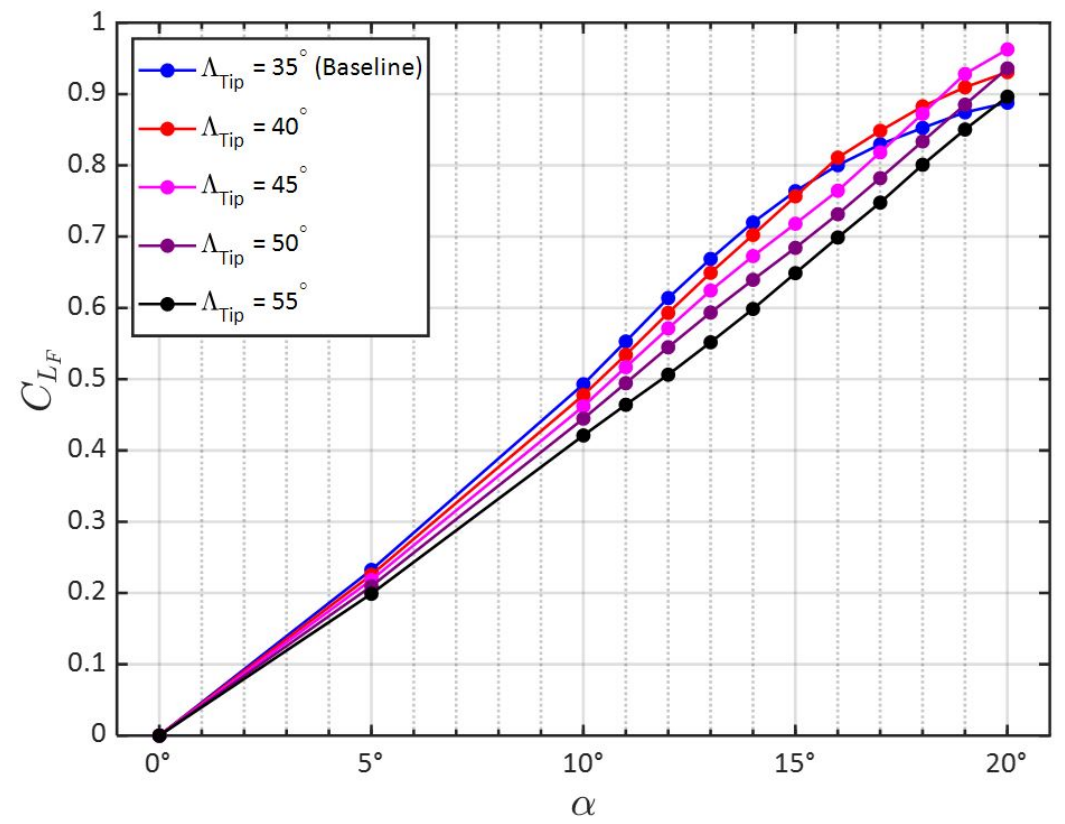

Figure 6.11: Lift curve comparison for $\Lambda_{t i p}=40^{\circ}, 45^{\circ}, 50^{\circ}$, and $55^{\circ}$.

\subsection{3 $\Lambda_{\text {tip }}=40^{\circ}, 45^{\circ}, 50^{\circ}, 55^{\circ}$ Lift, Shear and Vorticity}

The higher $\Lambda_{t i p}\left(\Lambda_{t i p}=40^{\circ}, 45^{\circ}, 50^{\circ}, 55^{\circ}\right)$ fin lift curves are shown in Figure 6.11. These fins showed similar trends to the baseline geometry, where there was no clear $C_{L_{F}, \max }$ point through $\alpha_{F}=20^{\circ}$. This reinforced the trend from subsection 6.2.2, that with an increase in sweep, there was a greater offset in separation and critical $\alpha_{F}$. The lift slope decreased as $\Lambda_{t i p}$ increased shown in Table 6.1.

Table 6.1: Lift curve slopes for high swept fin geometries.

\begin{tabular}{cccccc}
$\Lambda_{\text {tip }}$ & $35^{\circ}$ & $40^{\circ}$ & $45^{\circ}$ & $50^{\circ}$ & $55^{\circ}$ \\
\hline$a_{0}$ & 0.0492 & 0.0477 & 0.0461 & 0.0444 & 0.0420
\end{tabular}

The skin friction coefficient was also compared in Figure 6.12. The $\Lambda_{t i p}=40^{\circ}, 45^{\circ}$, $50^{\circ}, 55^{\circ}$ fins show similar streaks of shear areas exceeding $C_{F}=1.6 \times 10^{-2}$, indicating again that there was a vortex running past the surface at this location. The location of this high shear area remained above $50 \%$ span for each geometry, and the strength 


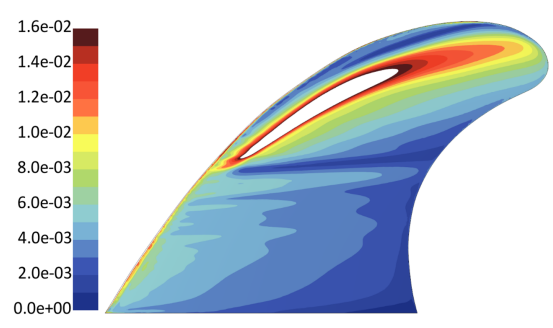

(a) $\Lambda_{t i p}=35^{\circ}$ (baseline)

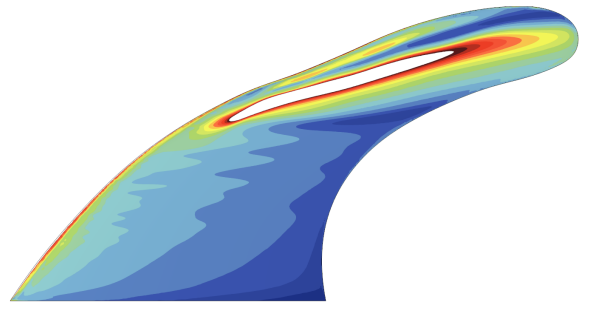

(d) $\Lambda_{t i p}=50^{\circ}$

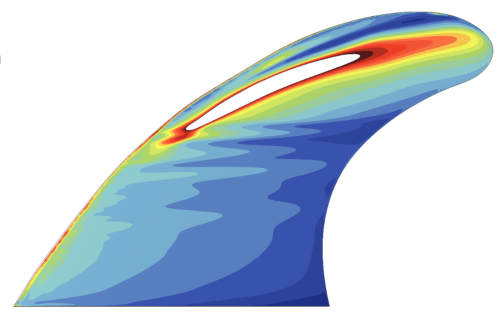

(b) $\Lambda_{t i p}=40^{\circ}$

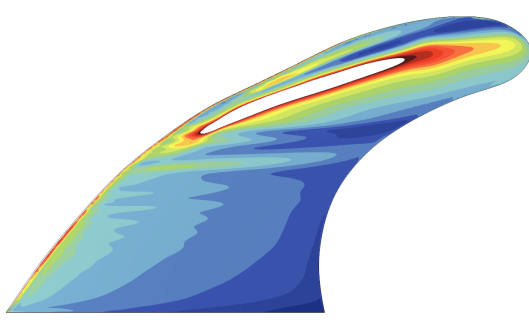

(c) $\Lambda_{t i p}=45^{\circ}$

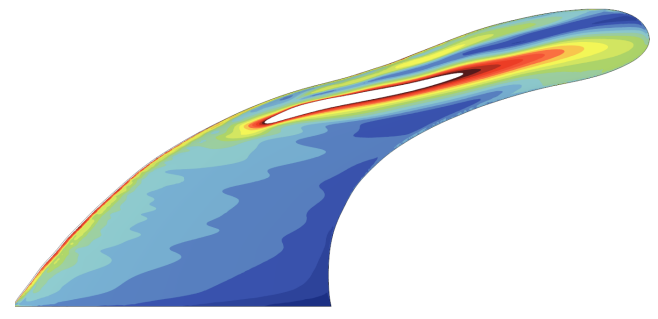

(e) $\Lambda_{\text {tip }}=55^{\circ}$

Figure 6.12: $\alpha_{F}=10^{\circ}$, skin friction coefficient comparison on the low pressure surface for the fins with increasingly more sweep.

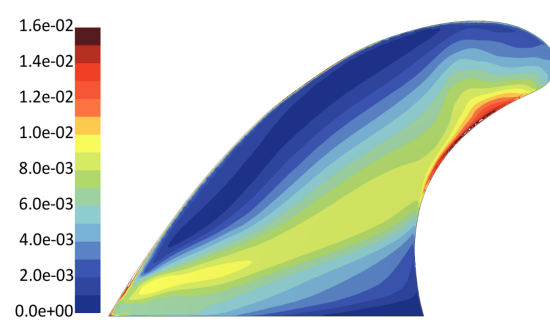

(a) $\Lambda_{t i p}=35^{\circ}$ (baseline)

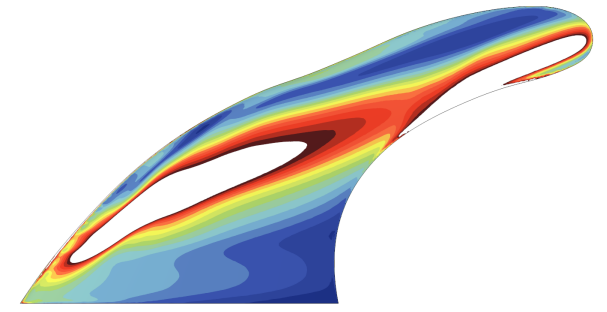

(d) $\Lambda_{t i p}=50^{\circ}$

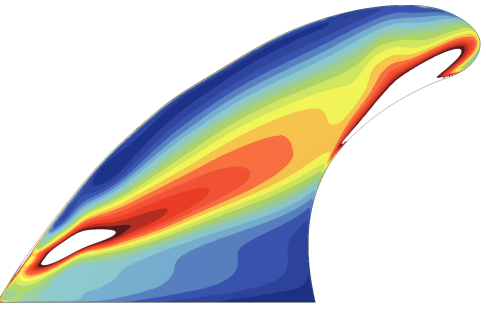

(b) $\Lambda_{t i p}=40^{\circ}$

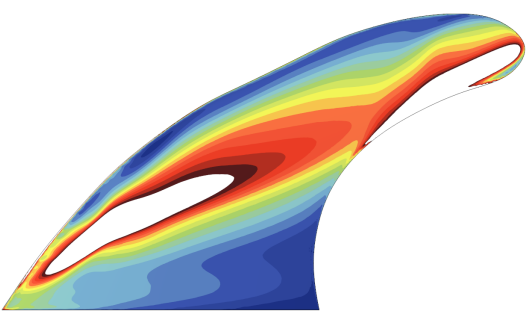

(c) $\Lambda_{t i p}=45^{\circ}$

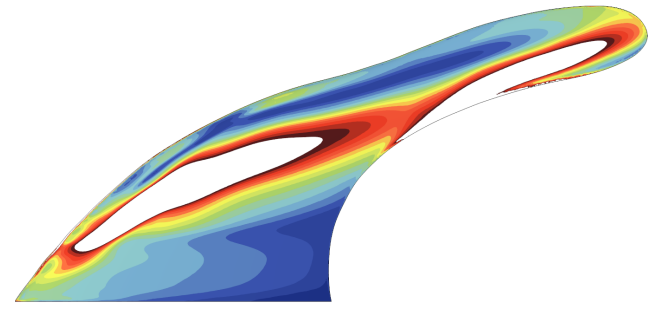

(e) $\Lambda_{\text {tip }}=55^{\circ}$

Figure 6.13: $\alpha_{F}=20^{\circ}$, skin friction coefficient comparison on the low pressure surface for the fins with increasingly more sweep.

and pattern remains consistent.

This was a clear difference from the previous fin set of $\Lambda_{t i p}=15^{\circ}, 20^{\circ}, 25^{\circ}, 30^{\circ}$. The decrease in sweep resulted in lower shear stress from the generated vortex, and 


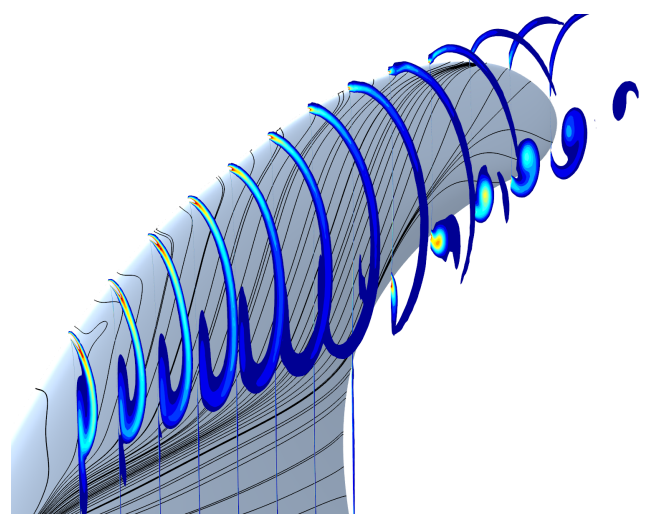

(a) $\Lambda_{\text {tip }}=40^{\circ}$

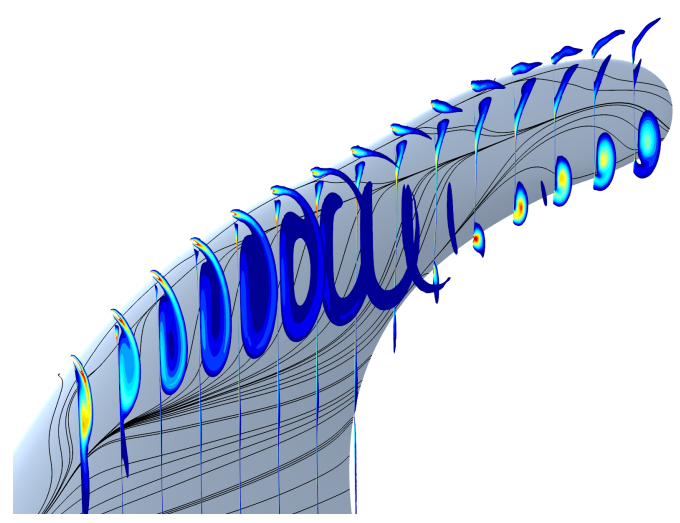

(c) $\Lambda_{\text {tip }}=50^{\circ}$

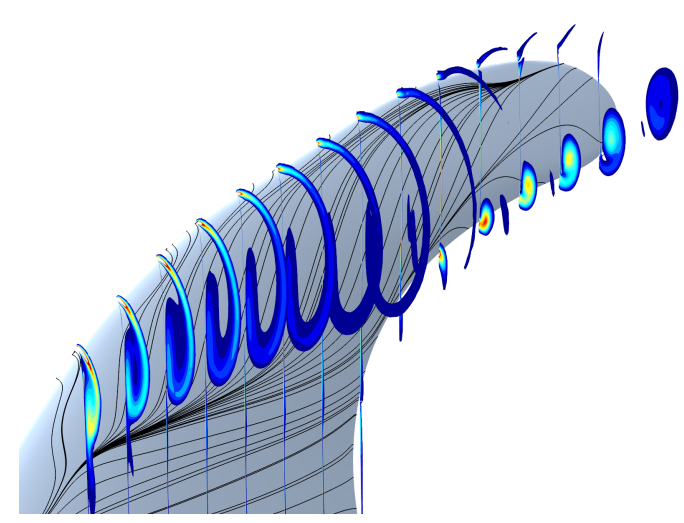

(b) $\Lambda_{\text {tip }}=45^{\circ}$

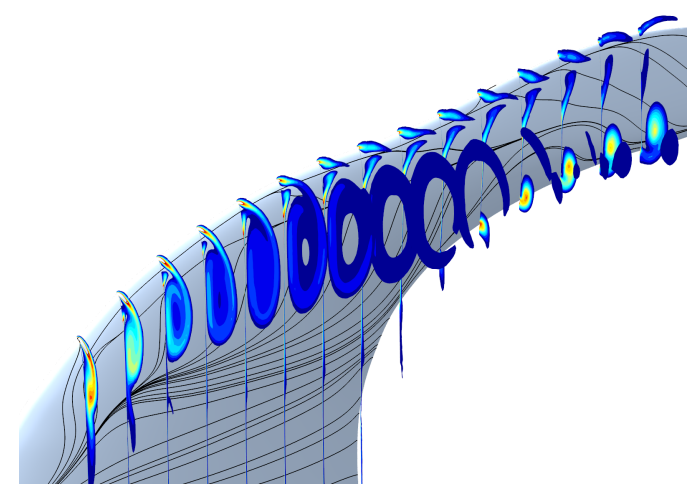

(d) $\Lambda_{\text {tip }}=55^{\circ}$

Figure 6.14: $\alpha_{F}=20^{\circ}$, vorticity images for $\Lambda_{t i p}=40^{\circ}$ through $55^{\circ}$.

the vortex dissipated faster. The higher $\Lambda_{t i p}$ fins showed the opposite trend. As can be seen in Figure 6.13 at $\alpha_{F}=20^{\circ}, \Lambda_{\text {tip }}=40^{\circ}, 45^{\circ}, 50^{\circ}$ and $55^{\circ}$ all have skin friction contours exceeding $C_{F}=1.6 \times 10^{-2}$, and additionally, there was also a secondary high shear region that develops towards the fin tip and near the trailing edge.

When the vorticity was investigated for these cases in Figure 6.14, it was clear that a second vortex formed. Moving from the leading edge to the trailing edge of $\Lambda_{t i p}=40^{\circ}, 45^{\circ}, 50^{\circ}$ and $55^{\circ}$ in Figure 6.14 , the initial vortex that forms off the leading edge begins to dissipate, but the low pressure generated from this vortex entrains the flow from aft portions of the fin, which then generated a new vortex off the fin tip.

The increased vorticity with increased $\Lambda_{\text {tip }}$ was a possible source for the differ- 
ences in $C_{L_{F}}$ curves in Figure 6.11. This is a trend classically more common with changing aspect ratio than with changing sweep because changing aspect ratio effects the downwash produced. Downwash is the result of low pressure created from strong vortices. The pressure gradient generated reduces the effective angle of attack on a wing or fin and therefore reduces the amount of lift produced [31]. Higher aspect ratio wings produce less induced drag from vortices than low aspect wings, and in turn create less downwash. The result of this is that although a high aspect ratio wing will have a higher maximum lift coefficient, it will stall sooner because it's effective angle of attack stays higher.

This was similar to the result seen by changing the sweep distribution in the fins. The increased vorticity as a result of increased sweep promotes a delay in the amount of separation generated at higher angles.

\subsubsection{Drag Comparison for Swept Fins}

Figure 6.15a shows the comparison of pressure versus shear drag for $\Lambda_{\text {tip }}=15^{\circ}, 20^{\circ}, 25^{\circ}$ and $30^{\circ}$, and Figure $6.15 \mathrm{~b}$ shows the comparison for $\Lambda_{t i p}=40^{\circ}, 45^{\circ}, 50^{\circ}$ and $55^{\circ}$. For every fin geometry the shear drag is proportionally much smaller than the pressure drag, and there was a noticeable difference in pressure drag.

The fins with less sweep had consistently lower drag coefficients than both the baseline fin and fins with higher sweep at incidence angles past $\alpha_{F}=15^{\circ}$. This stemmed from the difference in vortex generation. The fins with smaller $\Lambda_{\text {tip }}$ values did not produce as strong or any vortices at lower angles, which reduced the amount of pressure drag created.

When both the shear and pressure drag components are added together, the total drag was noticeably different. Figure 6.16a presented the percent difference in total drag for the smallest and largest $\Lambda_{t i p}$ cases $\left(15^{\circ}, 55^{\circ}\right)$ as compared to the baseline 


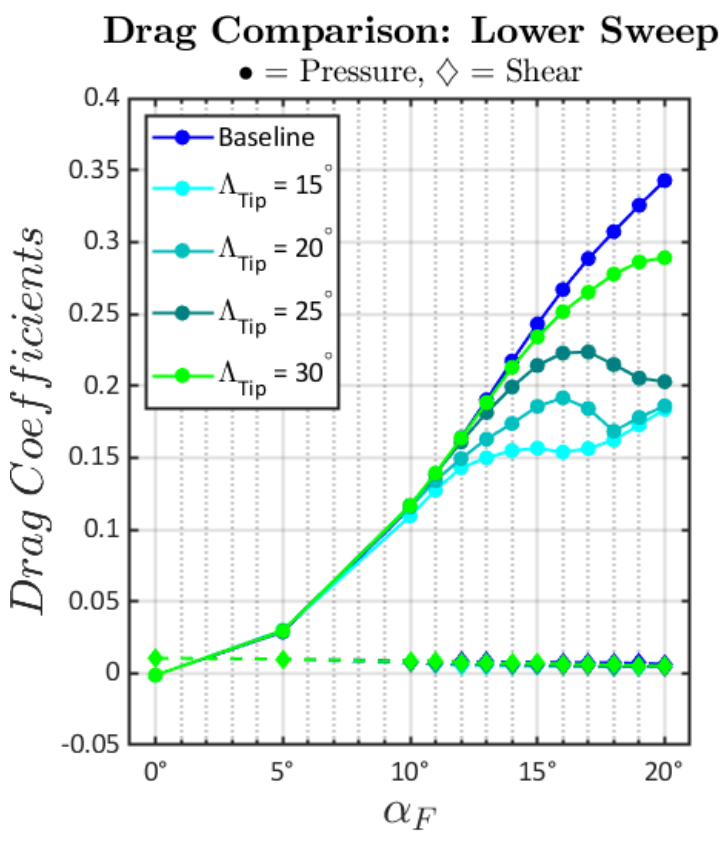

(a)

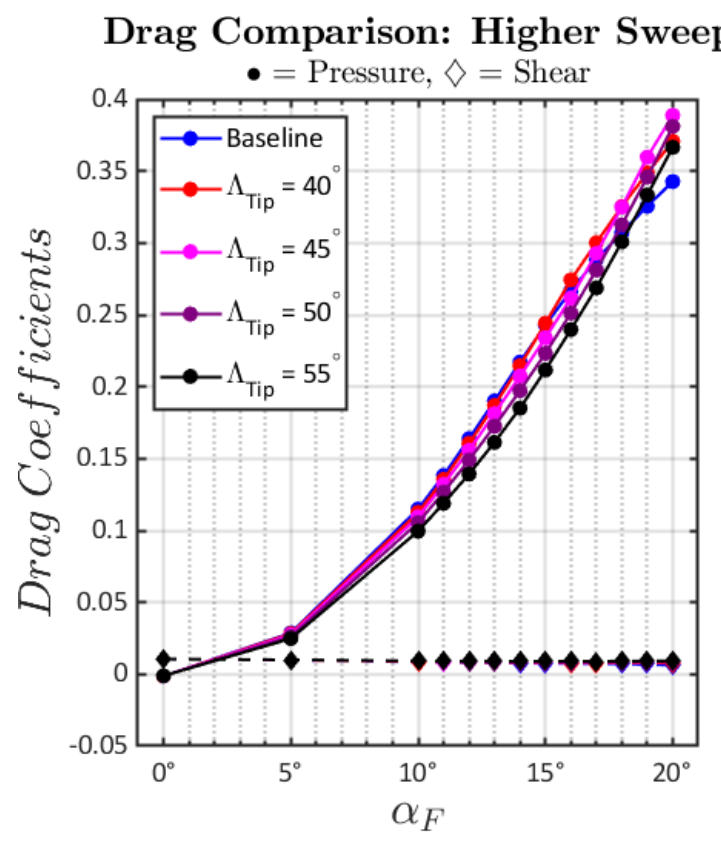

(b)

Figure 6.15: Pressure versus shear drag comparison for all $\Lambda_{t i p}$ values. The drag values are split into two plots: the lower and higher swept geometries.

geometry, where the percent difference is calculated as:

$$
\text { Percent Difference }(\%)=\frac{C_{D, \text { new }}-C_{D, \text { Baseline }}}{\left(C_{D, \text { new }}+C_{D, \text { Baseline }}\right) \cdot \frac{1}{2}} \cdot 100 \%
$$

Any percent difference value that is negative indicates a drag value less than the baseline configuration and vice versa. As $\alpha_{F}$ increases past $12^{\circ}$, there was a sizable gap in percent difference values, with a maximum difference between the two curves of $65.5 \%$ at $\alpha_{F}=19^{\circ}$. This equates to a difference in $C_{D}$ of 0.155 .

Even for smaller changes in sweep, the less swept geometry showed decreases in drag past $\alpha_{F}=12^{\circ}$. Figure 6.17 shows the same drag comparison but for the $\Lambda_{t i p}=$ $30^{\circ}$ and $40^{\circ}$ cases. Although the difference was not as great, after $\alpha_{F}=12^{\circ}$ the $\Lambda_{t i p}=30^{\circ}$ case begins to have distinctly lower total drag. At $\alpha_{F}=20^{\circ}$, the percent difference between the $\Lambda_{t i p}=30^{\circ}$ and $40^{\circ}$ cases is $25.9 \%$ with a difference in $C_{D}$ of 0.0841 . 


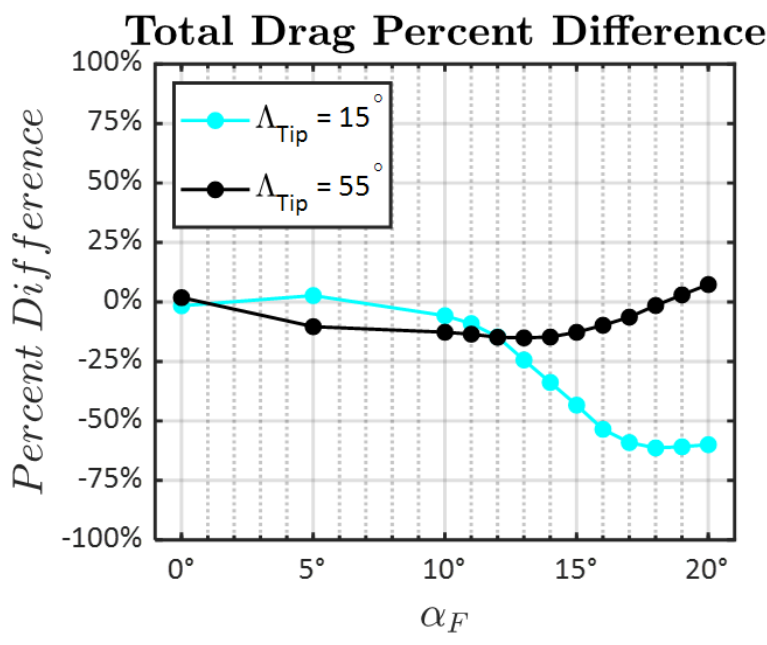

(a)

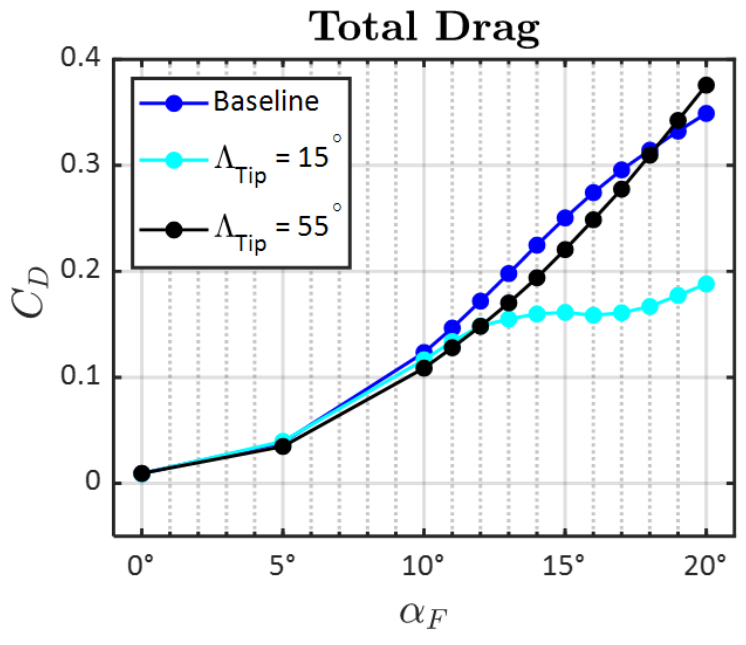

(b)

Figure 6.16: Drag comparison for $\Lambda_{t i p}=15^{\circ}$ and $55^{\circ}$ to the baseline geometry.

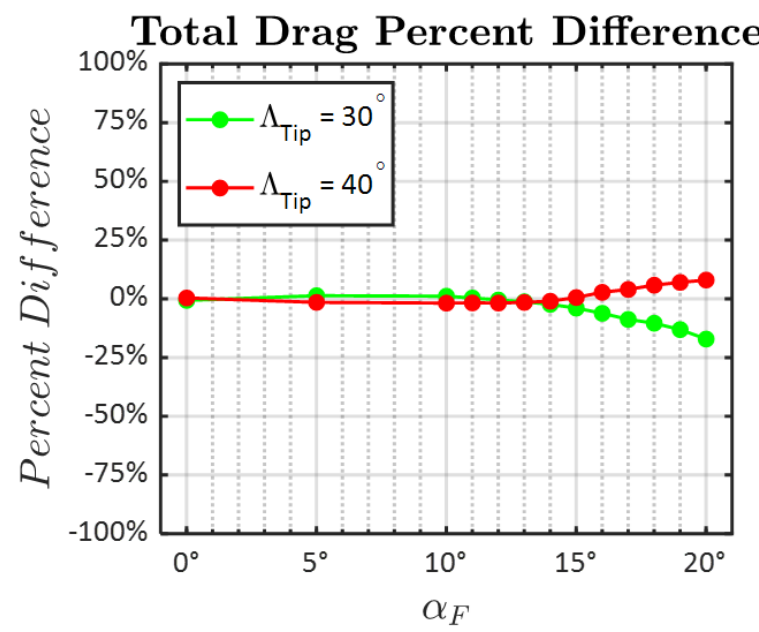

(a)

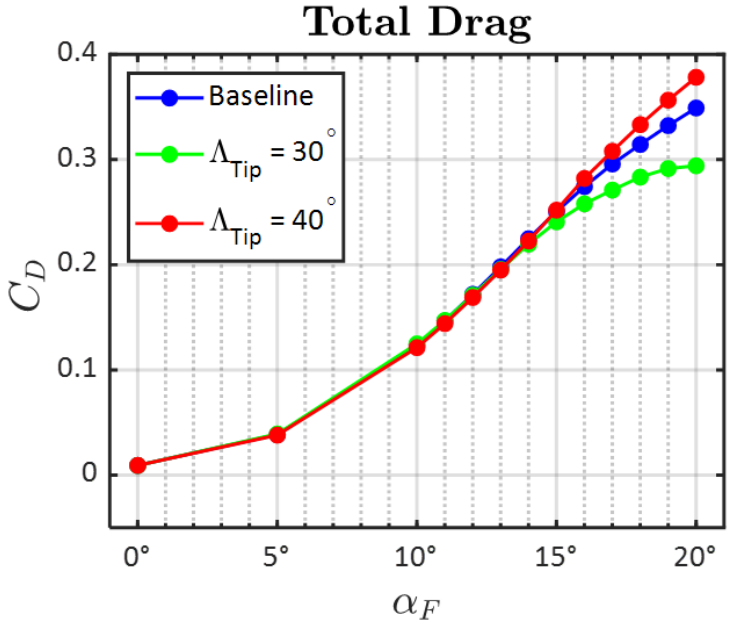

(b)

Figure 6.17: Drag comparison for $\Lambda_{t i p}=30^{\circ}$ and $40^{\circ}$ to the baseline geometry.

\subsubsection{Moment Comparison for Swept Fins}

The last metric used for comparison was the amount of moment each fin generated. From Equation 1.6, the moment was calculated by multiplying a moment arm to the lift force generated. The moment arm is relative to the center of gravity of the surfer, and the $c g$ location is highly variable depending on the surfboard and surfer riding the 


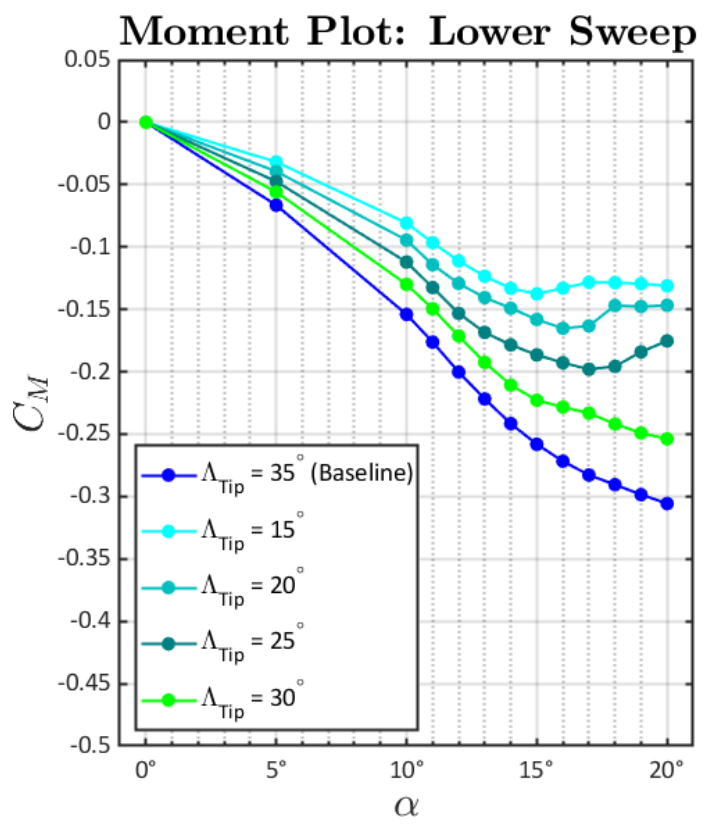

(a)

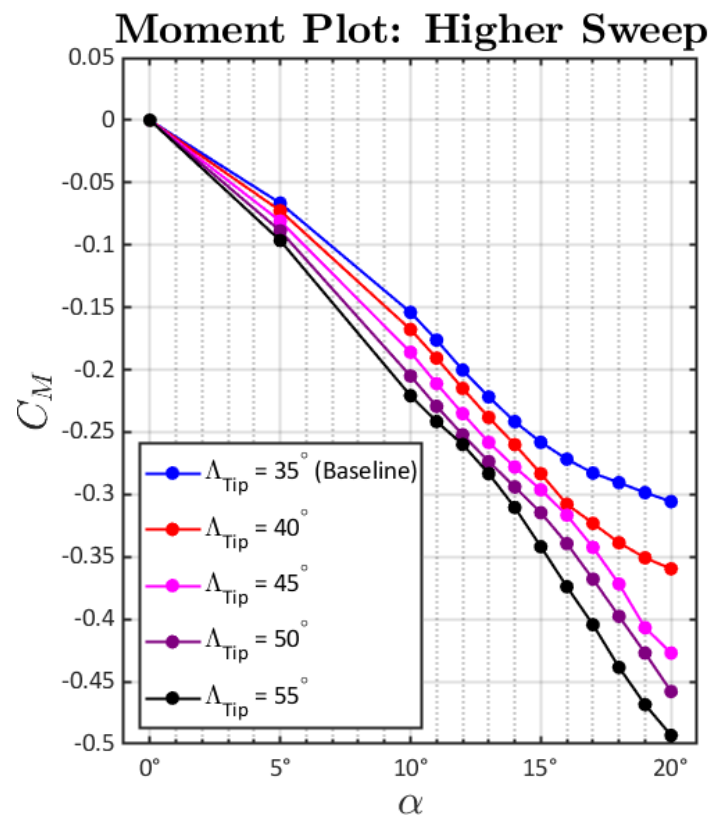

(b)

Figure 6.18: Moment coefficient comparison for all $\Lambda_{t i p}$ values. The drag values are split into two plots: the lower and higher swept geometries.

board, so 1.0 m was used as the moment arm for ease of calculation and an estimated distance on a shortboard.

Figure 6.18 presents the moment coefficient curves for each swept fin geometry. As the sweep distribution was increased, the moment coefficient increased as well. This was the result of two things. The first was that the fins with greater $\Lambda_{t i p}$ values generated more lift force and did not experience any drop in lift at higher $\alpha_{F}$.

The second reason was as each fin geometry increased in sweep, the moment arm increased as well. As the sweep increased, each hydrofoil cross section moved farther aft, and in doing so, increased the distance that the hydrodynamic center was located from the center of gravity. Since the hydrodynamic center was the point where the lift force was integrated too, this increased the moment arm and the amount of moment created. 


\subsection{Taper}

\subsubsection{Taper Geometry}

Figure 6.19 shows the results of the geometry creation using Equation 4.15. Each fin geometry will be referred to be the taper coefficient used to change the distribution, $K_{\text {taper }}$, and the fins with $K_{\text {taper }}$ values of -1.00 and 1.00 are the minimum and maximum differences in taper. The fins with negative taper coefficient have decreased surface area towards the root and increased area towards the tip, making them more rectangular and vice versa for the fins with positive taper coefficients, making them more triangular. Appendix B.3 shows more detail for each geometry.

Unfortunately, the amount of variation in this geometry set was not as great as the swept geometries. The positive $K_{\text {taper }}$ cases were very similar to the other positive

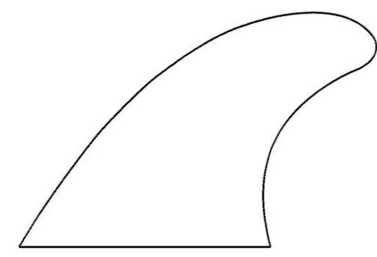

(a) $K_{\text {taper }}=0.00$ (Baseline)

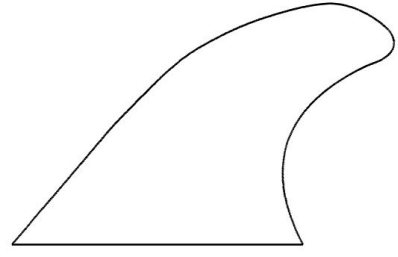

(b) $K_{\text {taper }}=0.50$

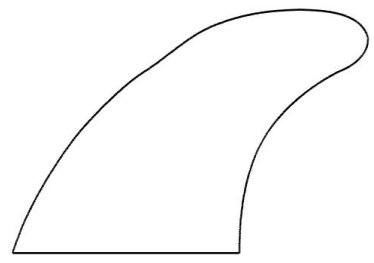

(e) $K_{\text {taper }}=-0.50$

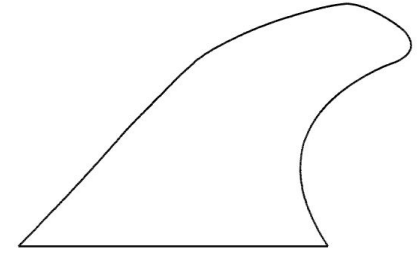

(c) $K_{\text {taper }}=0.75$

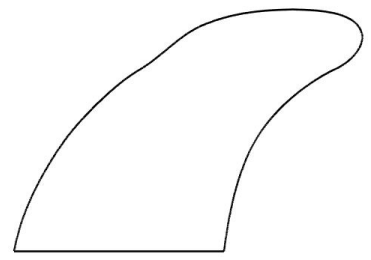

(f) $K_{\text {taper }}=-0.75$

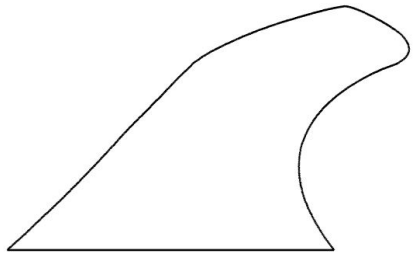

(d) $K_{\text {taper }}=1.00$

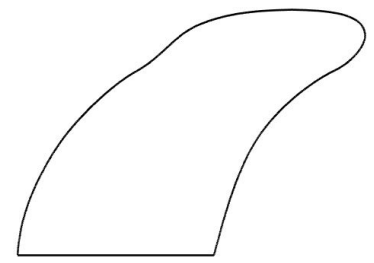

(g) $K_{\text {taper }}=-1.00$

Figure 6.19: Planform shapes of tapered geometries. 
cases, and the same for negative $K_{\text {taper }}$ fins. There is room to improve this geometry creation in the future, but for this research, the results from this geometry set show how sensitive changing the taper distribution is.

\subsubsection{Taper Lift, Shear, and Vorticity Comparison}

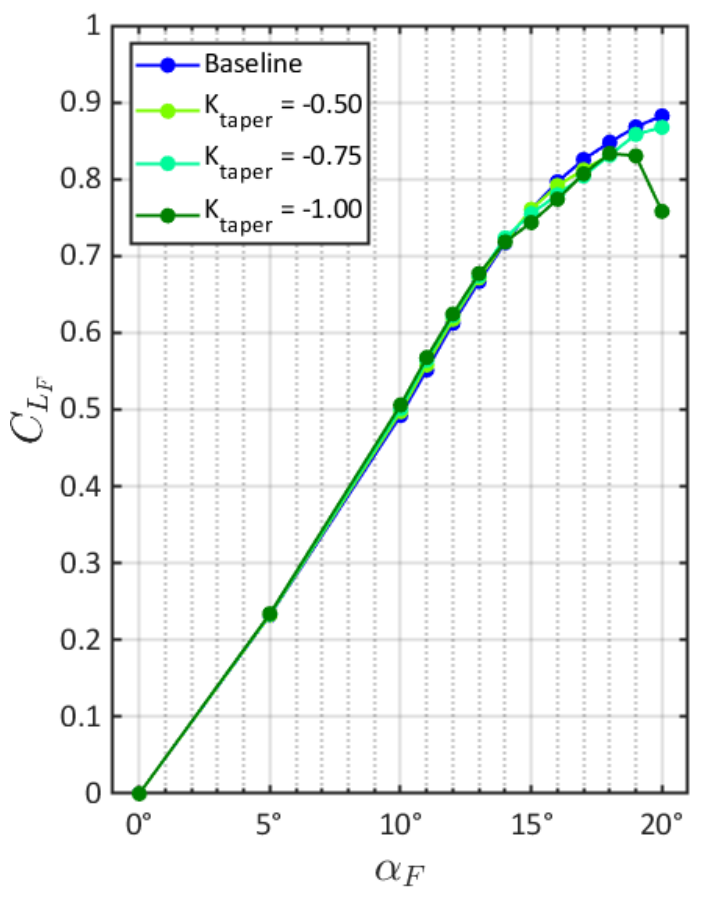

(a) $K_{\text {taper }}=-0.50,-0.75,-1.00$

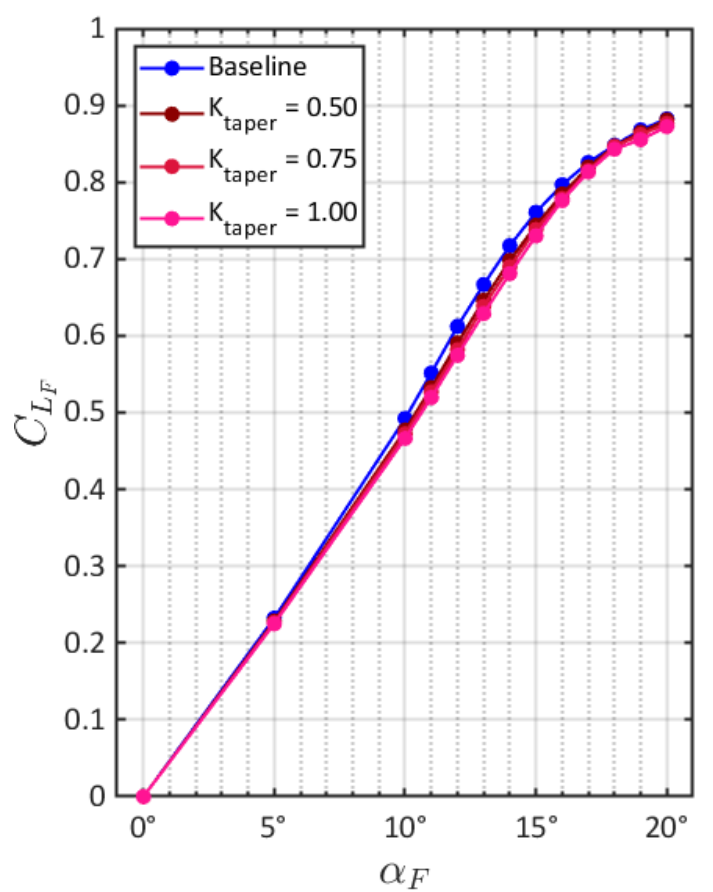

(b) $K_{\text {taper }}=0.50,0.75,1.00$

Figure 6.20: Tapered fin lift curve comparison.

A similar methodology was used to compare the tapered geometry to the baseline

fin. Lift curves were plotted for each tapered fin in Figure 6.20, and different than the swept geometries, the tapered fins show much closer trends to the baseline fin. Where in the swept cases, each $\Lambda_{t i p}$ value produced a different $C_{L, \max }$ point or lift curve slope; each $K_{\text {taper }}$ case was very similar. The only outlier was the $K_{\text {taper }}=-1.00$ case. Shown with the dark green line, this was the only line that has a distinct maximum lift point. 
Besides $K_{\text {taper }}=-1.00$, the rest of the tapered geometries overlapped the lift curve line set by the baseline geometry. Meaning, changing the taper distribution did not have as much of an effect on the lift force as changing the sweep. This was interesting as changing the taper often influences the stall and lift behavior. This is due to the change in cross sectional chord length. An increase or decrease in chord length influences the local Reynolds number and two dimensional lift produced at that location.

Figure 6.21 shows a comparison of the lift distributions for $K_{\text {taper }}=0.75$ and -0.75 to the baseline geometry in increments of $\alpha_{F}=5^{\circ}$. The change in taper distribution should shift the point of maximum $c_{l_{F}}$ depending on how the chord length is changed, but for both plots, this trend wasn't seen. At $\alpha_{F}=0^{\circ}, 5^{\circ}$, and $10^{\circ}$, the tapered geometries almost identically overlap the baseline fin, and at $\alpha_{F}=15^{\circ}$ and $20^{\circ}$ there are only two noticeable differences.

The first difference was in Figure 6.21a, $K_{\text {taper }}=0.75$. At $\alpha_{F}=20^{\circ}$, the $K_{\text {taper }}=$ 0.75 fin had a higher peak then the baseline geometry. The max value for the baseline lied at $\frac{y}{b}=0.2$ and was $c_{l_{f}} \cdot c=0.934$. For $K_{\text {taper }}=0.75$, the max was $c_{l_{f}} \cdot c=1.01$ and lied at $\frac{y}{b}=0.3$, a $10 \%$ change in height, but a percent difference of $7.82 \%$.

The second difference was with $K_{\text {taper }}=-0.75$ in Figure 6.21b. At $\alpha_{F}=15^{\circ}$, the $K_{\text {taper }}=-0.75$ lift distribution has its maximum lift values shifted closer to the root chord, but at $\alpha_{F}=20^{\circ}$ both plot lines return to following the same trend. The shift at $\alpha_{F}=15^{\circ}$ could indicate a shift in the vortex generation to be closer to the root chord, and when investigated in Figure 6.22 this appears to be true.

Figure 6.22 shows the skin friction coefficient contours on the suction surface of the baseline and tapered geometries for $\alpha_{F}=10^{\circ}, 15^{\circ}$ and $20^{\circ}$. Figure $6.22 \mathrm{k}$ shows the $K_{\text {taper }}=-0.75$ fin at $\alpha_{F}=15^{\circ}$, and when compared to the baseline geometry, the location of the high shear area was closer to the root chord at the same angle, 


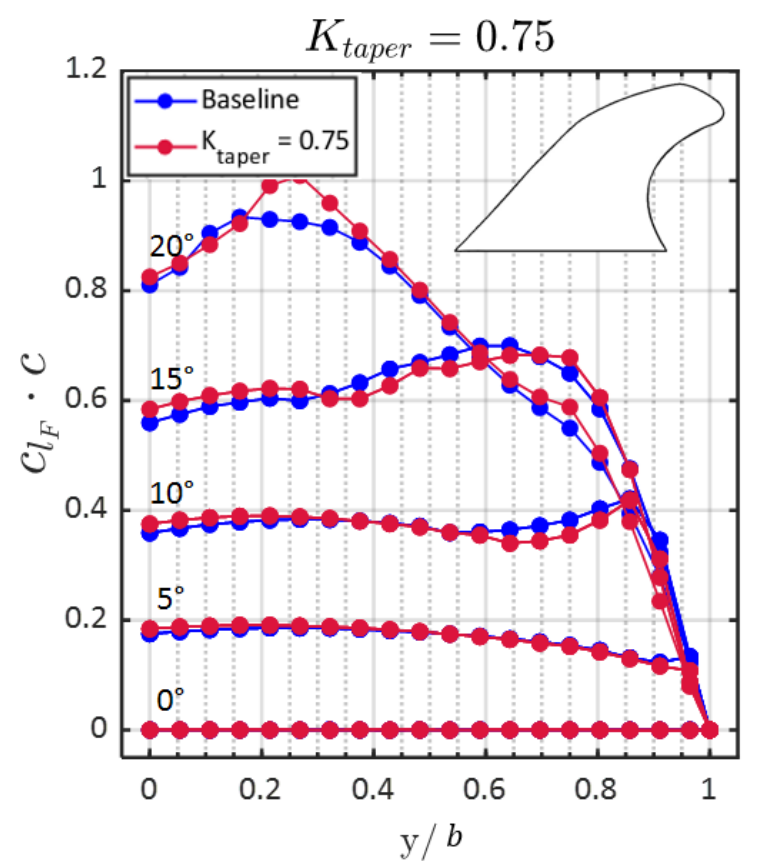

(a)

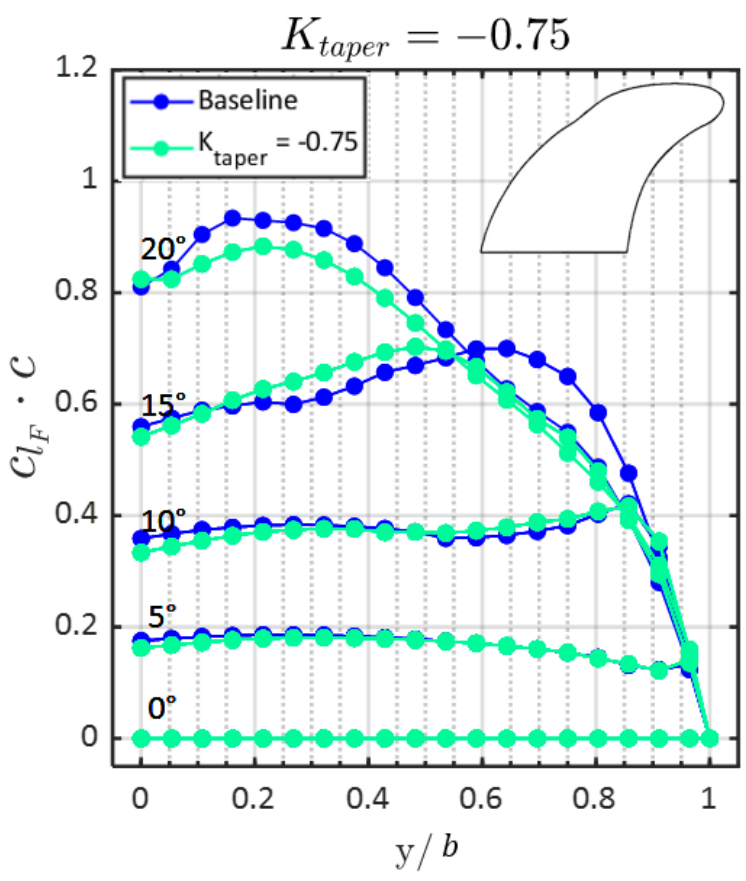

(b)

Figure 6.21: Lift distribution comparison for $K_{\text {taper }}=0.75$ and -0.75 to the baseline geometry. Each line represents the lift distribution for a given $\alpha_{F}$ in increments of $5^{\circ}$.

indicating a shift in the location of the vortex core. This trend appeared in the positive $K_{\text {taper }}$ cases as well, as the high shear areas are moved closer to the tip.

From Figure 6.22 there are a few other flow features that emerge. For both negative $K_{\text {taper }}$ fins at $\alpha_{F}=20^{\circ}$, there are no high skin friction regions. This means that for these fins the vortices have dissipated and switched to separated regions, which is not seen in the positive $K_{t}$ aper fins.

The other flow feature seen in Figure 6.22f and Figure 6.22i was a small high shear region exceeding $C_{F}=1.6 \times 10^{-2}$ that reforms near the leading edge. This was also highlighted by the lift distribution for $K_{\text {taper }}=0.75$ in Figure $6.21 \mathrm{~b}$ by the sharp peak in lift force at $\alpha_{F}=20^{\circ}$. This region was different than what has been seen previously in that it is focused to a single location. Previously, there was a streak that went across the surface of the fin due to a vortex traveling past the surface, 


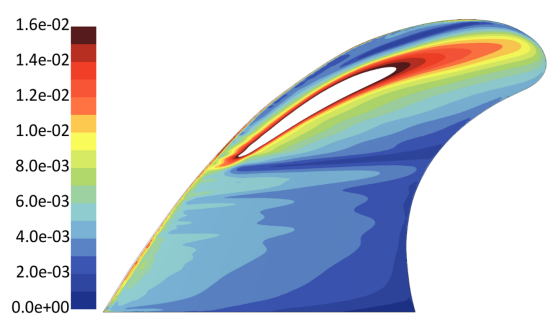

(a) Baseline, $\alpha_{F}=10^{\circ}$

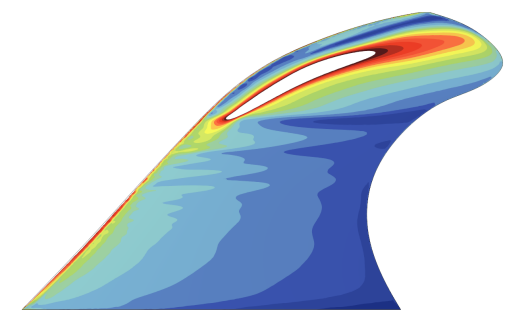

(d) $K_{\text {taper }}=0.75, \alpha_{F}=10^{\circ}$

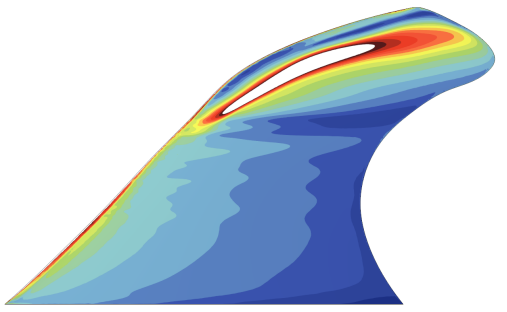

(g) $K_{\text {taper }}=1.00, \alpha_{F}=10^{\circ}$

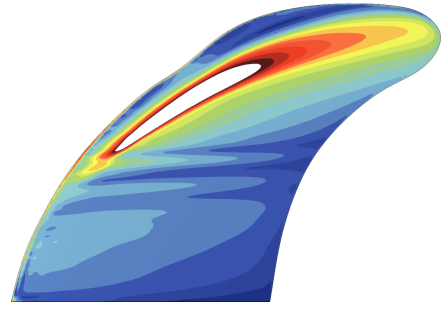

(j) $K_{\text {taper }}=-0.75, \alpha_{F}=10^{\circ}$

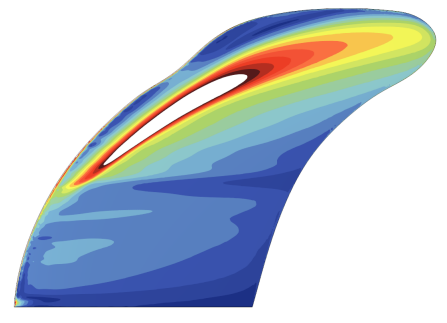

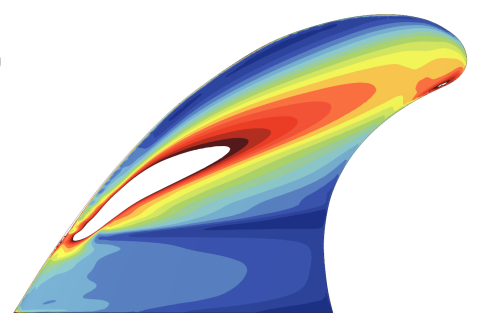

(b) Baseline, $\alpha_{F}=15^{\circ}$

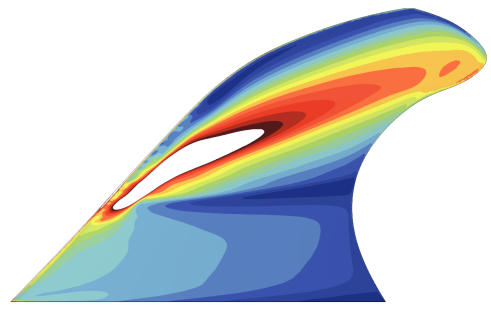

(e) $K_{\text {taper }}=0.75, \alpha_{F}=15^{\circ}$

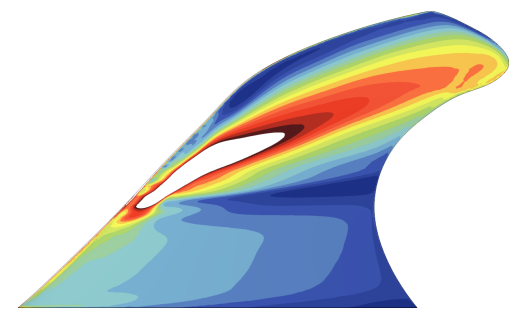

(h) $K_{\text {taper }}=1.00, \alpha_{F}=15^{\circ}$

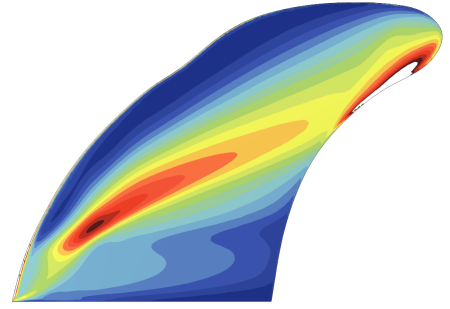

(k) $K_{\text {taper }}=-0.75, \alpha_{F}=15^{\circ}$

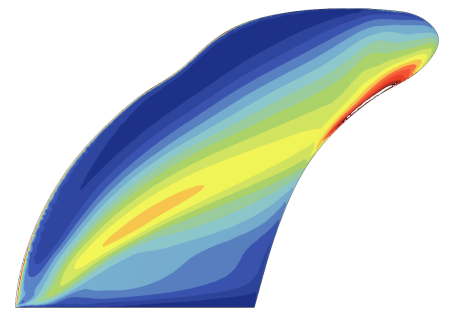

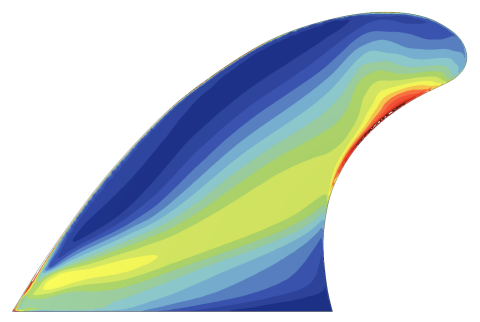

(c) Baseline, $\alpha_{F}=20^{\circ}$

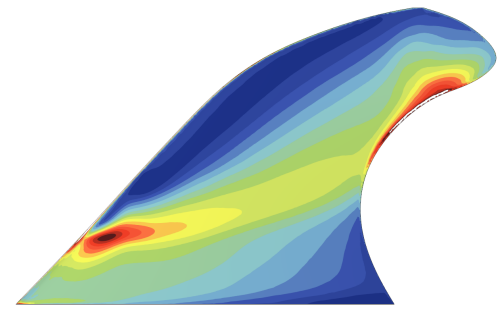

(f) $K_{\text {taper }}=0.75, \alpha_{F}=20^{\circ}$

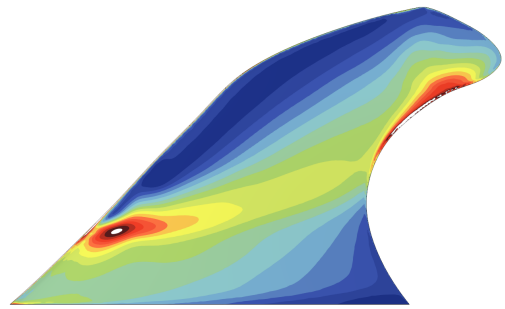

(i) $K_{\text {taper }}=1.00, \alpha_{F}=20^{\circ}$

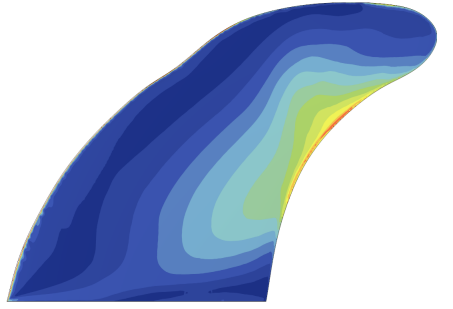

(l) $K_{\text {taper }}=-0.75, \alpha_{F}=20^{\circ}$

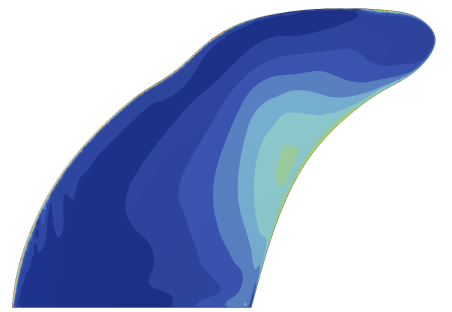

(m) $K_{\text {taper }}=-1.00, \alpha_{F}=10^{\circ}$

(n) $K_{\text {taper }}=-1.00, \alpha_{F}=15^{\circ}$

(o) $K_{\text {taper }}=-1.00, \alpha_{F}=20^{\circ}$

Figure 6.22: Skin friction coefficient comparison on the low pressure surface for the fins with different taper distributions. Areas of white on the fin surface indicate high shear areas that exceed the maximum scalar value. 


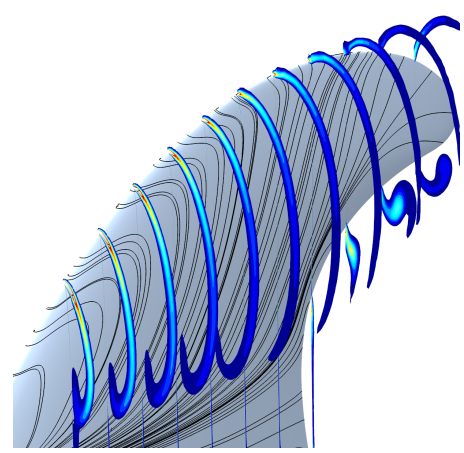

(a) Baseline

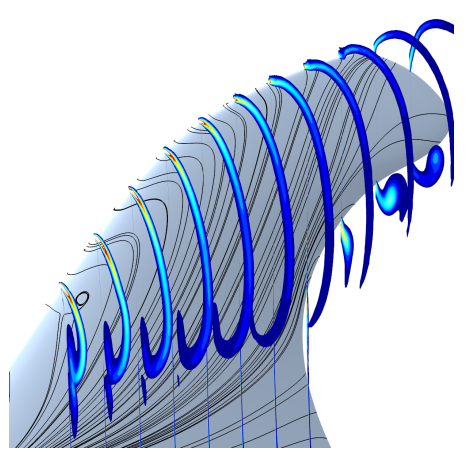

(b) $K_{\text {taper }}=0.75$

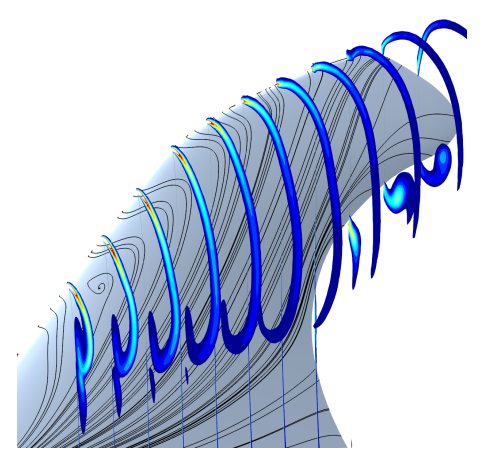

(c) $K_{\text {taper }}=1.00$

Figure 6.23: Vorticity comparison at $\alpha_{F}=20^{\circ}$ for positive $K_{\text {taper }}$ fins and the baseline geometry.

but as Figure 6.23 shows, at $\alpha_{F}=20^{\circ}$, there was not a vortex core at this location. Instead, the high shear area was caused by large amounts of acceleration. There was still separated flow that dominated the upper half of the surface, but close to the root chord there was still attached flow that was highly accelerated.

This was a feature unique to the positive $K_{\text {taper }}$ fins. Further geometry testing needs to be done to see if increasing the root chord was the cause of this.

\subsubsection{Taper Drag Comparison}

The drag force was also broken into pressure and shear drag coefficient components for the tapered fins and plotted in Figure 6.24. For the positive $K_{\text {taper }}$ fins, the drag coefficient was near identical to the baseline fin, but the negative $K_{\text {taper }}$ fins had lower pressure drag. This was due to a decrease in vortex strength. As the $K_{\text {taper }}$ constant became more negative, the fin became more rectangular. This seemed to reduce the strength of the vortex created off the leading edge, and in doing so, reduced the pressure drag coefficient. Besides this, the change in taper distribution had little influence on the drag coefficients. 
Drag Comparison: Positive $K_{\text {taper }}$ $\bullet=$ Pressure, $\diamond=$ Shear

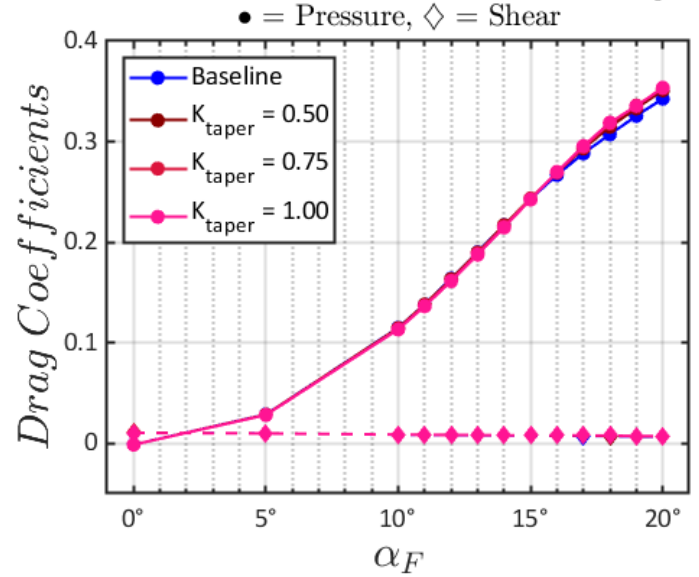

(a)
Drag Comparison: Negative $K_{\text {taper }}$

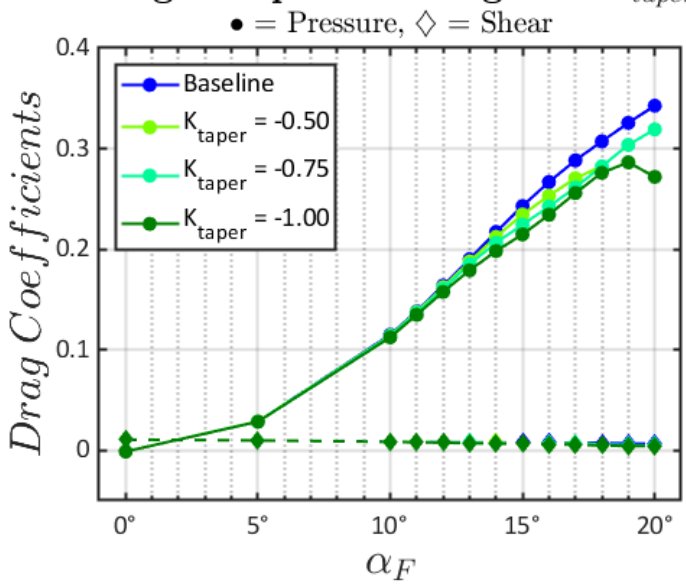

(b)

Figure 6.24: Pressure versus shear drag coefficient comparison for both positive and negative $K_{\text {taper }}$ fin geometries.

\subsubsection{Taper Moment Comparison}

The moment coefficient for each of the tapered geometries were plotted in Figure 6.25.

There was not much variation, which was expected, as the lift curves were also very similar.

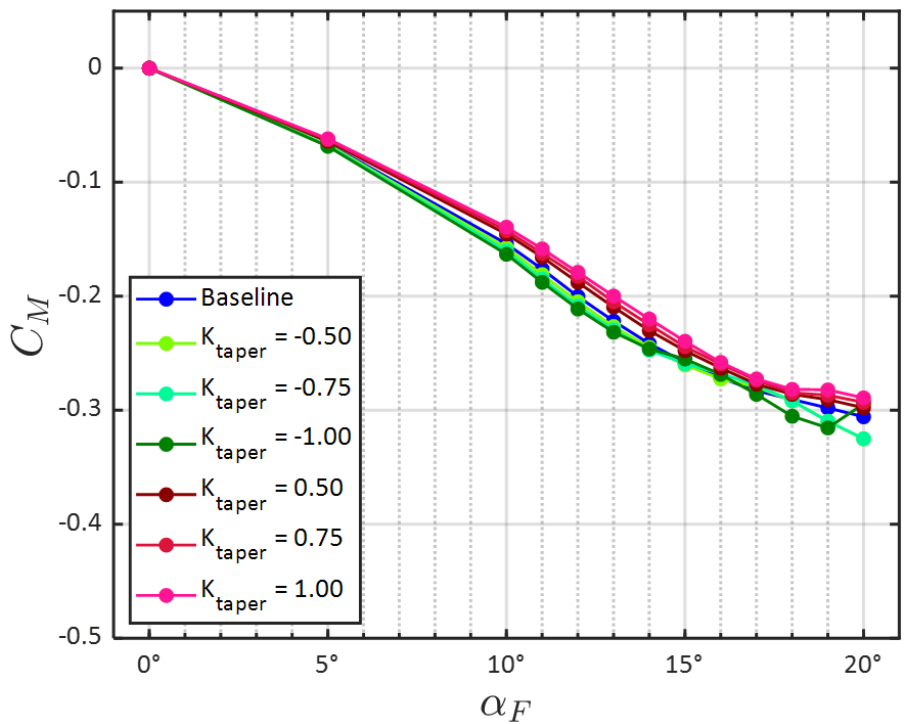

Figure 6.25: Moment coefficient comparison for each of the tapered geometries. 


\section{Chapter 7}

\section{CONCLUSIONS}

\subsection{Effectiveness of Geometry Creation}

The geometry creation process focused on altering the sweep and taper of a given baseline planform whilst maintaining the quarter-chord line and aspect ratio of the fin. This was to isolate the effects of changing either how swept or tapered a fin was without the influence of other geometric parameters. However, neither the sweep or taper were constant along the span of the fin, so the sweep and taper distribution with respect to the span were varied instead.

The sweep distribution was successfully altered to create eight different fin outlines. This was done by translating each cross section proportionally to a desired final tip sweep value. This methodology maintained the other geometric parameters of the fin and increased or decreased the sweep distribution. It proved to be effective, but the simplicity of the methodology became apparent at the maximum and minimum $\Lambda_{\text {tip }}$ values, where the leading edge become distorted. Future iterations of this method should enforce conditions that maintain the concavity of the leading edge.

Changing the taper distribution was not as well executed. There was an inherent problem with the baseline geometry in that the $M H C$ occurred at more than one location along the span. This meant that the taper distribution did not uniformly increase or decrease, so the same kind of proportional change was not possible. Instead an equation was developed (Equation 4.15) that maintained the length and location of the $M H C$ and increased or decreased the chord lengths above or below it. This was partially successful. There were a limited range of coefficients that could be used in this equation, and the resultant planform shapes were not overly different, but 
using Equation 4.15, six different geometries were created and simulated.

If further studies were to be done on the taper of surfboard fins, the taper equation needs to become more robust. It is possible that the issue is inherent with the baseline geometry and multiple $M H C$ locations, but this could be checked with a fin that does not have multiple $M H C$ locations, or by creating an alternate equation that only enforces a single $M H C$ location.

There were an additional four geometries created using a separate, proportional equation (Equation 4.14). These cases were not included in the body of the paper, as this method changed the surface area of the fin. The results from the proportional method, however, can be seen in Appendix B.4.

\subsection{Effects of Sweep and Taper on Performance}

After each of the fin shapes were created, they were simulated in CFD using the RANS equations at $R e=3.51 \times 10^{5}$ from $\alpha_{F}=0^{\circ}$ to $20^{\circ}$. The results from these simulations showed that changing the sweep distribution influenced a vortex formation across the suction surface of the fin. The baseline shape showed a strong vortex that formed on and followed the leading edge, and as the sweep distribution decreased this vortex either did not form or dissipated at a lower incidence angle. When the sweep distribution increased the vortex persisted for higher angles. This influenced the fin lift, drag and moment coefficients.

Through $\alpha_{F}=12^{\circ}$, the lower swept fins maintained higher lift values, but began to see points of separation and stall. Although the lift curve slope was smaller, the fins with more sweep maintained increasing values for longer. The fin lift force, however, is not always the most influential metric to a surfer's performance. A fin on a surfboard is not used to counteract a weight force, but to aid in maneuvering the surfboard, and the moment coefficient is a better indicator of how effective the fin is at doing 
this.

It was clear that increasing the sweep distribution on the fin increased the moment generated. One of the largest influences to this was the fact that the hydrodynamic center of the fin is moved aft as the sweep increased, increasing the moment arm. This came at a cost of increased drag. At $\alpha_{F}<12^{\circ}$, the higher swept fins have similar if not lower drag values, but as $\alpha_{F}$ is increased, there are large increases and differences in drag. This ultimately means a few things for a surfing with differently swept fins.

A fin with lower sweep will be better for maintaining speed through turns. It may not be able to turn as quickly as a higher swept fin, but at larger incidence angles there is a huge savings in drag. This could be extremely useful for surfing a larger faced wave, where the surfer needs to accelerate quickly past the initial drop and turn past the breaking part of the wave or has room to make large sweeping maneuvers. Fins with higher sweep will excel at making sharp hard turning maneuvers, but need either a lot of speed beforehand or a fast moving wave. This is also assuming the fins hold perfect rigidity, which is much more difficult to do with the higher swept fins.

There are fewer conclusive statements to be made about changing the taper distribution. The positive $K_{\text {taper }}$ fins increased the root chord and decreased the tip chord, making the fin more triangular, and had very similar characteristics to the negative $K_{\text {taper }}$ cases. The only points of difference where that at higher incidence angles, the negative $K_{\text {taper }}$ fins had lower drag values, but the positive $K_{\text {taper }}$ fins delayed separation for longer.

\subsection{Future Work}

There is still much work to be done for surfing hydrodynamics. As the sport continues to become more popular, the effectiveness of the equipment will become more important. This research serves as foundation for understanding the geometric influences 
on fin design, and as such needs a follow-up study to validate the results found. The most important flow feature that needs validation is the vortex formation along the leading edge. This seemed to be a predictable feature influenced by the fin sweep. Water tunnel testing could confirm that this is indeed influenced by the fin geometry and not manufactured by the meshing or simulation environment.

If the results were confirmed, further studies on tapering the fins should be done along with testing new baseline geometries.

Ultimately, the simulations and testing on fins need to be tested in a more realistic surfing environment. In order to determine how much a fin influences the performance of surfboard both the board and the correct flow field need to be included. However, the fluid environment around a surfboard is dynamically changing and difficult to imitate. Simulating this is computationally expensive, and the flow field is rarely constant. Although it is difficult to do this, it will be an important next step to understanding how the shape of the surfboard fin affects the performance. 


\section{BIBLIOGRAPHY}

[1] FCS Fin Data. https://www.surffcs.com/pages/fcs-fin-data. accessed: 2018-06-20.

[2] Tom Blake. Tom Blake's Nub-Keel Fin. Surfer Magazine, 22(3), 1981. http://www.surfresearch.com.au/f.html.

[3] Mark Blackburn. Surfs up: collecting the longboard era. Schiffer Pub., 2001.

[4] P A Brandner and G J Walker. Hydrodynamic Performance of a Surfboard Fin. In 15th Australasian Fluid Mechanics Conference, 2004.

[5] Tony Butt. Surf science: an introduction to waves for surfing. Univ. of Hawaii Press, third edition, 2014.

[6] Boardcave. Concave's, flats and vee's and how they affect your surfboard. https://www . boardcave. com/the-surfers-corner/surfboard-bottomcontours/, 2015. accessed: 2018-11-22.

[7] John David Anderson. Fundamentals of aerodynamics. McGraw-Hill Education, 2017.

[8] D. R. Philpott and R. H. Barnard. Aircraft Flight, chapter 2. Prentice Hall, third edition, 2011.

[9] Vincent W. Tong and Richard J. Millard. Wind Tunnel Method and Analysis of Surfboard Fins. 2006.

[10] Megan S Macneill. Bio-Inspired Optimal Fin Shape and Angle for MaximumSurfboard Stability. 2015. 
[11] FCS II Filipe Toledo Tri Fins.

https://www.surffcs.com/products/fcs-ii-filipe-toledo-tri-fins. accessed: 2018-06-20.

[12] Surfing Heritage Foundation. The History of Surfing. http://files.surfingheritage.org/flash/timeline. accessed: 2018-07-14.

[13] Joel K. Bourne. Inside the Curl: Surfing's Surprising History. https://news .nationalgeographic.com/news/2013/08/130803-surfingsurprising-history-hawaiian-culture-extreme-sports/, 2013. accessed: 2018-09-28.

[14] A Greenough Scrapbook. The Australian Surfer's Journal, 2(2), 1999. http://www. surfresearch.com.au/f.html.

[15] FCS II Julian Wilson PC Aircore Limited Tri Fins. https://www .surffcs.com/collections/fins/products/fcs-ii-julianwilson-pc-aircore-limited-tri-fins. accessed: 2018-11-21-18.

[16] Magicseaweed. Bob \& Sink: The Trial and Error of Ryan Burch. https://magicseaweed.com/news/bob-sink-the-trial-and-error-ofryan-burch/6167/, 2014. accessed: 2018-11-21.

[17] Paul Kvinta. Surfonomics 101. http://fortune.com/2013/06/05/surfonomics-101/, 2013. accessed: 2018-11-21.

[18] Ryan Brower. The science behind how Kelly Slater's wave pool churns out perfect waves. https:

//www.adventuresportsnetwork.com/sport/surf/the-science-behindhow-kelly-slaters-wave-pool-churns-out-perfect-waves-video/, 2017. accessed: 2018-11-21. 
[19] World Surf League. Rules and regulations. http: //www . worldsurfleague.com/pages/rules-and-regulations, 2018. accessed: 2018-11-25.

[20] Anderson Jr. John D. Aircraft Performance and Design. McGraw-Hill, 1999.

[21] How to Choose Surf Fins: Fin Setup and Types. https://www . evo.com/guides/how-to-choose-surf-fins. accessed: 2018-11-11.

[22] Guide to Surfboard Fins. https://www.tactics.com/info/guide-to-surfboard-fins. accessed: 2018-11-11.

[23] P. Gudimetla, N. Kelson, and B. E. Analysis of the hydrodynamic performance of three and four surfboards using computational fluid dynamics. Australian Journal of Mechanic Engineering, 7(1), 2009.

[24] Magicseaweed. Are Quads Better Than Thrusters. https:

//magicseaweed.com/news/are-quads-better-than-thrusters/4379/, 2009. accessed: 2018-11-21.

[25] Oliver Farley, Nigel Harris, and Andrew Kilding. Physiological Demands of Competitive Surfing. Journal of Strength and Conditiong Research, 26(7):1887-1896, 2012.

[26] Engineering Toolbox. Sea water properties. https: //www.engineeringtoolbox.com/sea-water-properties-d_840.html, 2005. accessed: 2018-06-10.

[27] Michael V Cook. Flight dynamic principles a linear systems approach to aircraft stability and control. Butterworth-Heinemann, third edition, 2007. 
[28] Siemens. STAR-CCM+User Manual. Siemens.

[29] Rusty Preisendorfer. Talking Design: The Board Blog with Rusty Preisendorfer. http://www.surfline.com/blogs/talking-design-theboard-blog-with-rusty-preisendorfer/rusty-breaks-down-theingredients-for-a-perfect-four-fin_38677/, 2009. accessed: 2018-11-25.

[30] Frank M. White. Viscous fluid flow. McGraw-Hill Higher Education, third edition, 2011.

[31] NASA. Downwash effects on lift. https://www.grc.nasa.gov/www/k-12/airplane/downwash.html. accessed: 2018-06-10.

[32] S.M. Salim and S.C Cheah. Wall y+ Strategy for Dealing with Wall-bounded Turbulent Flows. Proceedings of the International MultiConference of Engineers and Computer Scientists, 2, 2009. 


\section{APPENDICES}

Appendix A

TABULATED DATA

\section{A.1 Sampled Fin Measurements}

\begin{tabular}{ccccc}
\hline Fin Name & Type of Fin & $t(\mathbf{m m})$ & $c(\mathbf{m m})$ & $t / c$ \\
\hline Futures Jordy Small & Side & 6.94 & 111.23 & $6.24 \%$ \\
\hline Futures AM1 & Side & 7.57 & 111.92 & $6.76 \%$ \\
\hline Futures Control Pyzel Series & Side & 6.00 & 117.20 & $5.12 \%$ \\
\hline FCS II Performer & Side & 7.30 & 109.66 & $6.66 \%$ \\
\hline FCS II Carver & Side & 6.90 & 110.63 & $6.24 \%$ \\
\hline FCS ARC & Side & 7.97 & 115.40 & $6.91 \%$ \\
\hline FCS PC-7 & Side & 7.03 & 114.07 & $6.16 \%$ \\
\hline FCS GMB-5 & Center & 7.53 & 111.30 & $6.77 \%$ \\
\hline FCS II FW & Side & 7.11 & 110.25 & $6.45 \%$ \\
\hline Futures Machado & Side & 6.70 & 111.60 & $6.00 \%$ \\
\hline Futures Machado & Center & 6.94 & 108.59 & $6.39 \%$ \\
\hline Futures AM2 HC Thruster & Center & 7.15 & 114.82 & $6.23 \%$ \\
\hline Futures AM2 HC Thruster & Side & 7.06 & 115.50 & $6.11 \%$ \\
\hline Futures John John Florence & Side & 7.03 & 110.24 & $6.38 \%$ \\
\hline Futures John John Florence & Center & 7.55 & 111.11 & $6.80 \%$ \\
\hline FCS II FW Five Fin & Outer & 7.17 & 108.87 & $6.59 \%$ \\
\hline FCS II FW Five Fin & Side & 7.70 & 106.00 & $7.26 \%$ \\
\hline FCS II FW Five Fin & Center & 7.35 & 101.45 & $7.24 \%$ \\
\hline
\end{tabular}




\begin{tabular}{ccccc}
\hline FCS II Performer (X-large) & Side & 7.19 & 114.10 & $6.30 \%$ \\
\hline FCS II Performer (X-large) & Center & 7.55 & 114.80 & $6.58 \%$ \\
\hline FCS II MF & Side & 6.90 & 113.00 & $6.11 \%$ \\
\hline FCS II MF & Center & 6.93 & 113.68 & $6.10 \%$ \\
\hline
\end{tabular}

Table A.1: Dimensions of surfboard fins. There is one outer fin. This is for the five fin setup, which uses a thruster configuration with the addition of two outer fins

\section{A.2 Mesh Settings}

Table A.2: Mesh sizing for the domain. The domain size represents the cell size of the volume mesh away from the surfboard fin, and the growth rate determines how fast the cells in the domain increase in size.

\begin{tabular}{ccc} 
Iteration & Domain Cell Size $(\mathbf{m})$ & Growth Rate \\
\hline 1 & 0.50 & 1.00 \\
\hline 2 & 0.50 & 0.85 \\
\hline 3 & 0.25 & 0.70 \\
\hline 4 & 0.35 & 0.85 \\
\hline 5 & 0.35 & 0.70 \\
\hline 6 & 0.50 & 0.50 \\
\hline 7 & 0.50 & 0.50 \\
\hline 8 & 0.50 & 1.00 \\
\hline 9 & 0.50 & 1.00 \\
\hline
\end{tabular}


Table A.3: Mesh sizing for the wake behind the fin. The wake length represents the length of the refinement area aft of the fin, the wake angle determines the spread of the refinement, and the wake density determines the target cell size in the wake.

\begin{tabular}{cccc}
\hline Iteration & Wake Length & Wake Angle & Wake Density \\
\hline 1 & 1.00 & $10^{\circ}$ & 0.060 \\
\hline 2 & 3.00 & $20^{\circ}$ & 0.030 \\
\hline 3 & 5.00 & $30^{\circ}$ & 0.015 \\
\hline 4 & 3.00 & $20^{\circ}$ & 0.030 \\
\hline 5 & 3.00 & $20^{\circ}$ & 0.020 \\
\hline 6 & 3.00 & $20^{\circ}$ & 0.030 \\
\hline 7 & 3.00 & $20^{\circ}$ & 0.030 \\
\hline 8 & 3.00 & $20^{\circ}$ & 0.040 \\
\hline 9 & 3.00 & $20^{\circ}$ & 0.040 \\
\hline
\end{tabular}

Table A.4: Mesh sizing for the fin surface size. The fin surface size is the targeted mesh size on the fin surface and the minimum surface size is the allowed minimum size for cells wrapping around curvature.

\begin{tabular}{ccc} 
Iteration & Fin Surface Size & Fin Minimum Surface Size \\
\hline 1 & 0.0500 & $1.00 \times 10^{-2}$ \\
\hline 2 & 0.0250 & $1.00 \times 10^{-2}$ \\
\hline 3 & 0.0050 & $5.00 \times 10^{-3}$ \\
\hline 4 & 0.0175 & $7.00 \times 10^{-3}$ \\
\hline 5 & 0.0175 & $3.50 \times 10^{-3}$ \\
\hline 6 & 0.0175 & $8.75 \times 10^{-4}$ \\
\hline 7 & 0.0175 & $1.75 \times 10^{-3}$ \\
\hline 8 & 0.0175 & $2.10 \times 10^{-3}$ \\
\hline 9 & 0.0175 & $2.50 \times 10^{-3}$ \\
\hline
\end{tabular}


Appendix B

FIN DATA

\section{B.1 Baseline Fin}
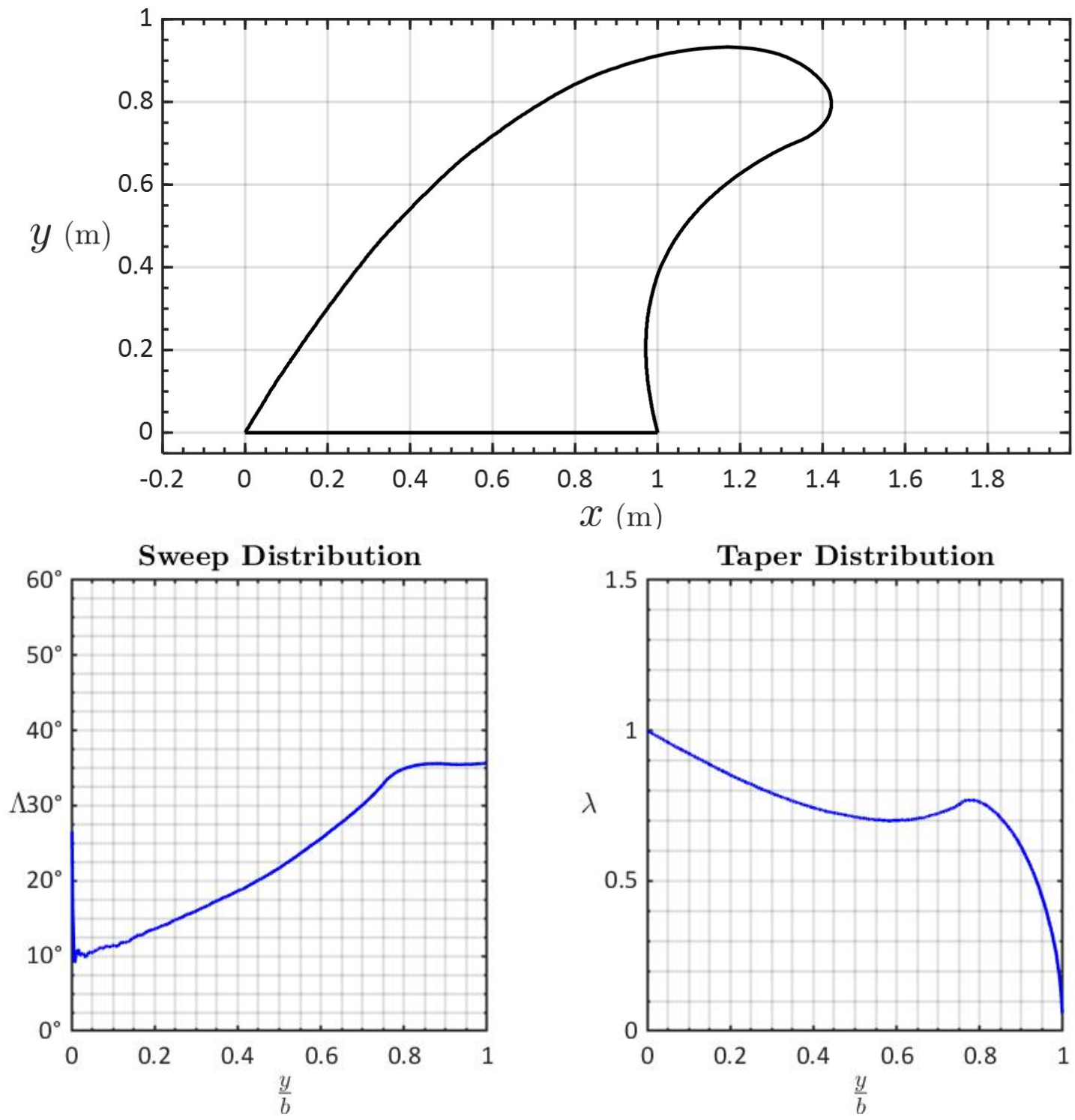

Figure B.1: Planform characteristics, baseline fin. 

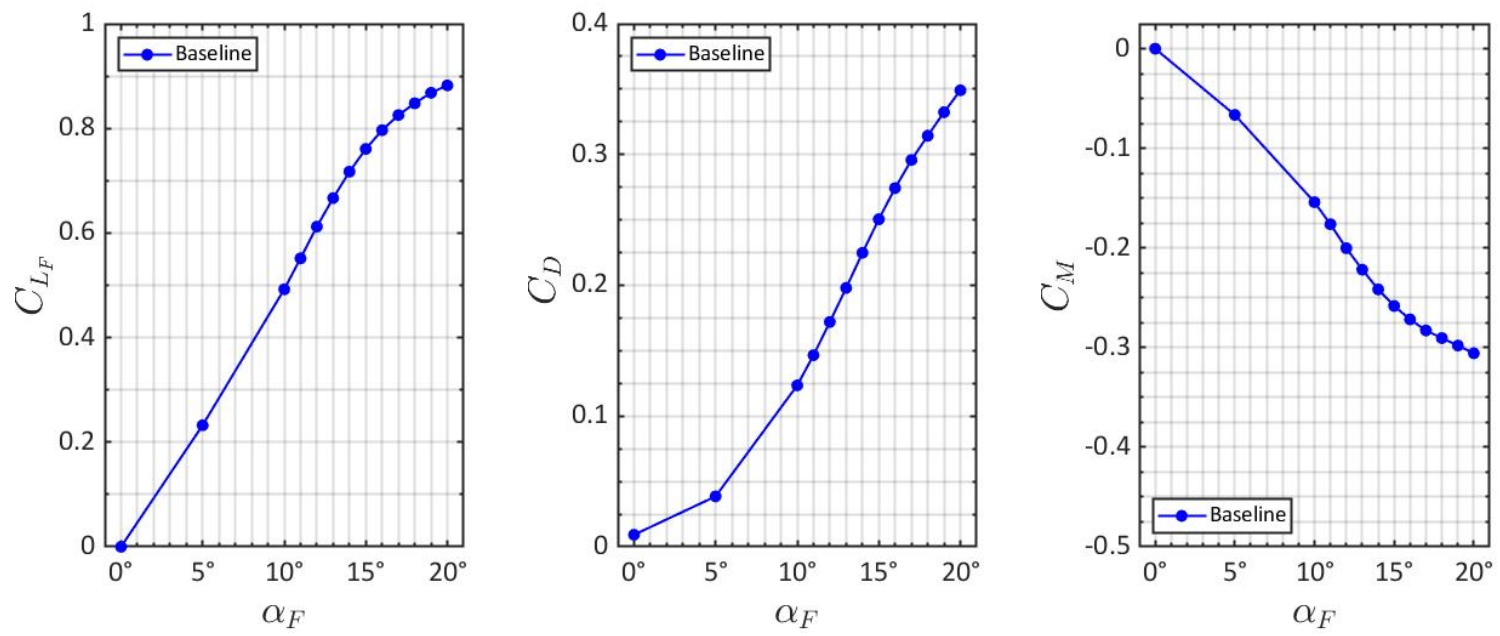

Figure B.2: Lift, drag, and moment coefficients, baseline fin.

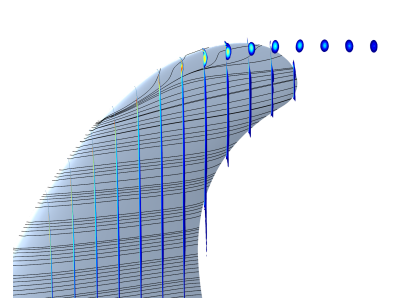

(a) $\alpha_{F}=5^{\circ}$

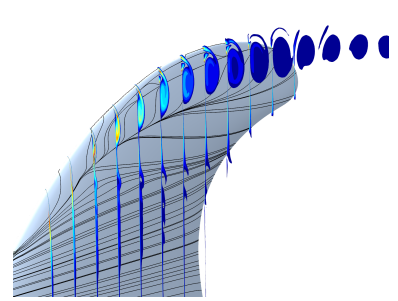

(b) $\alpha_{F}=10^{\circ}$

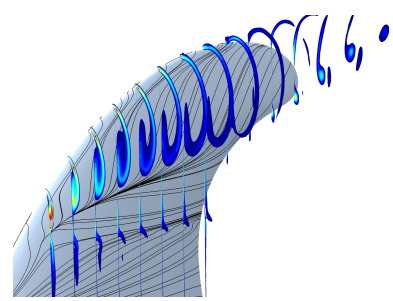

(c) $\alpha_{F}=15^{\circ}$

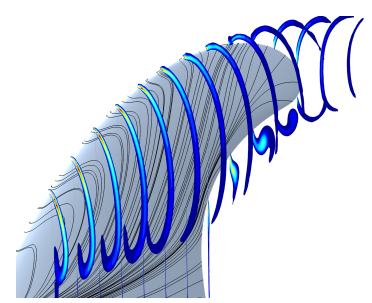

(d) $\alpha_{F}=20^{\circ}$

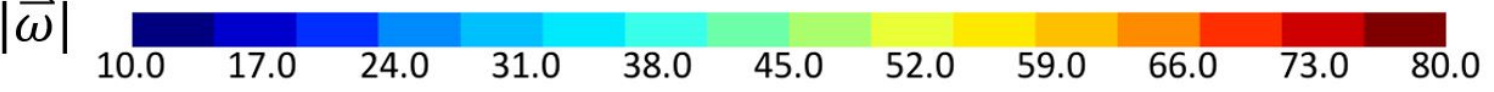

Figure B.3: Vorticity cross sections on the suction surface, baseline fin.

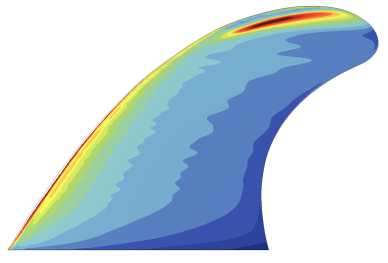

(a) $\alpha_{F}=5^{\circ}$

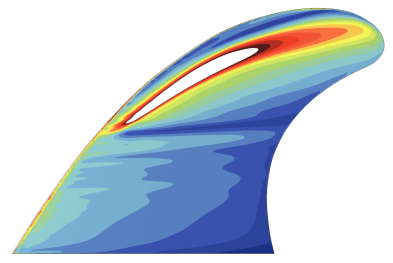

(b) $\alpha_{F}=10^{\circ}$

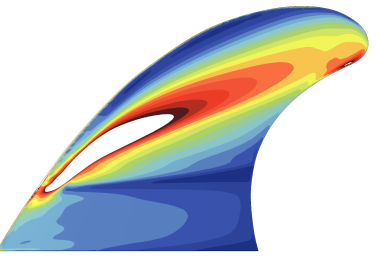

(c) $\alpha_{F}=15^{\circ}$

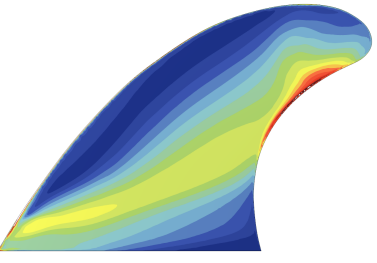

(d) $\alpha_{F}=20^{\circ}$

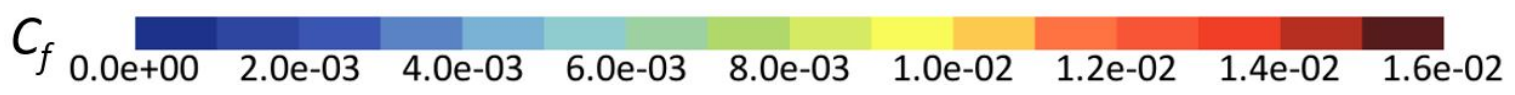

Figure B.4: $C_{f}$ coefficient contours on the suction surface, baseline fin. 


\section{B.2 Sweep}

B.2.1 $\Lambda_{t i p}=15^{\circ}$
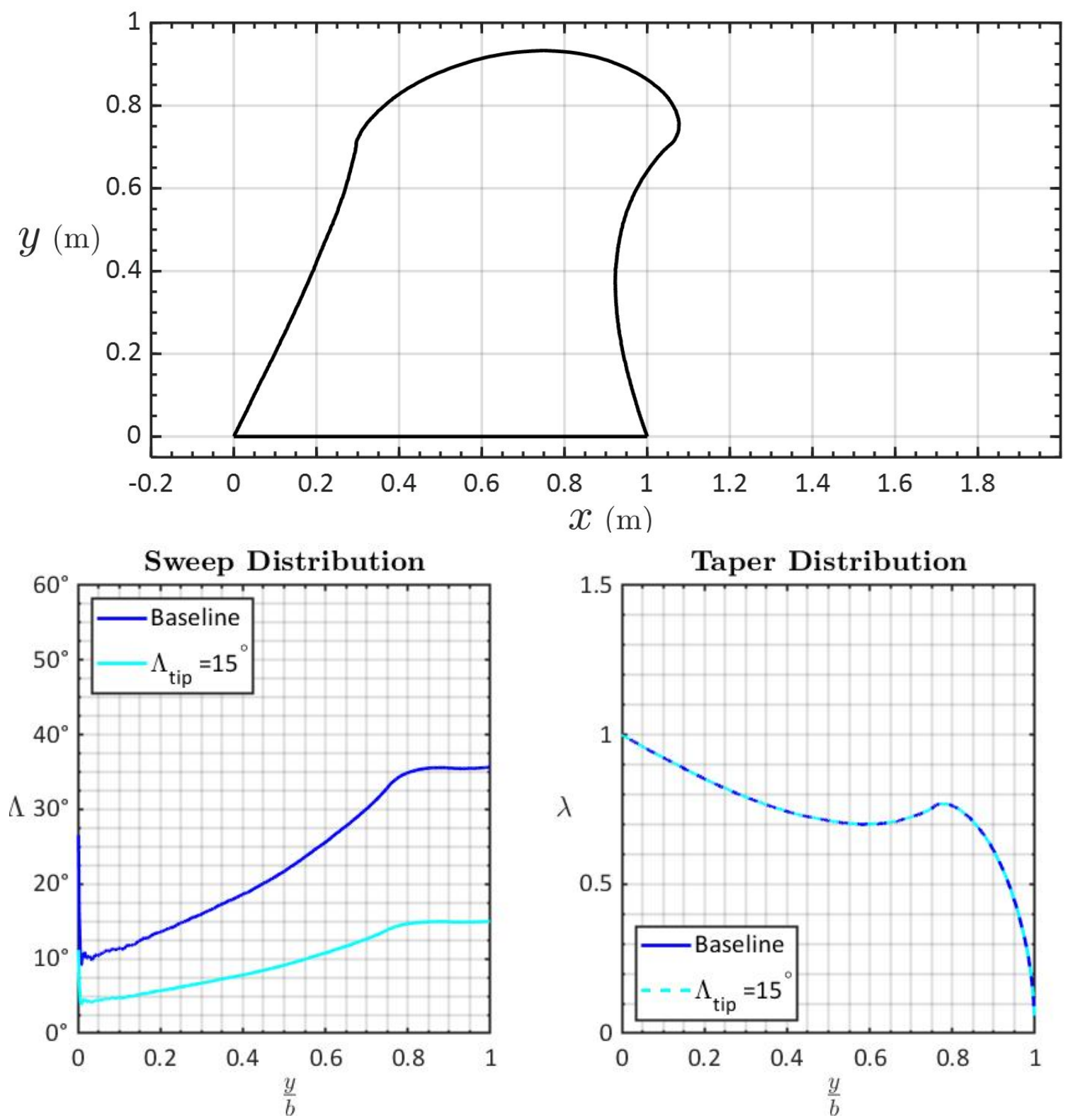

Figure B.5: Planform characteristics, $\Lambda_{t i p}=15^{\circ}$. 

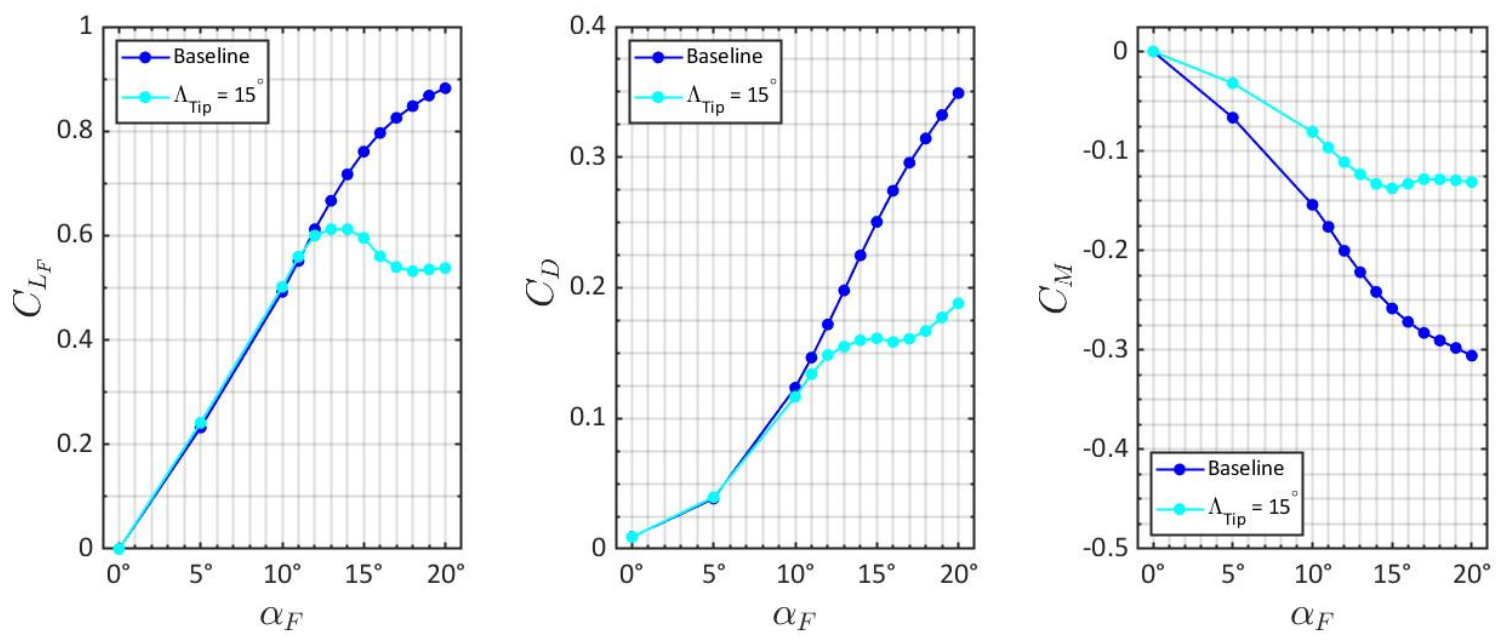

Figure B.6: Lift, drag, and moment coefficients, $\Lambda_{t i p}=15^{\circ}$.

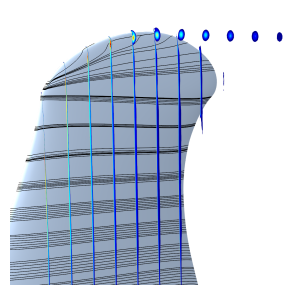

(a) $\alpha_{F}=5^{\circ}$

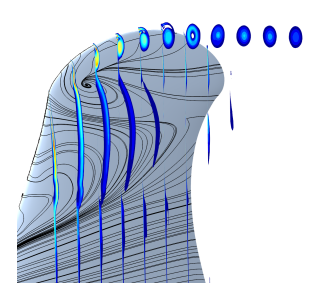

(b) $\alpha_{F}=10^{\circ}$

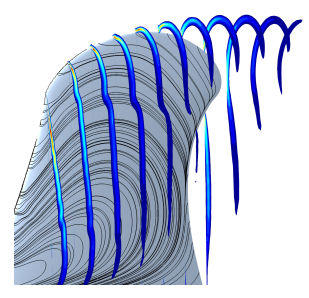

(c) $\alpha_{F}=15^{\circ}$

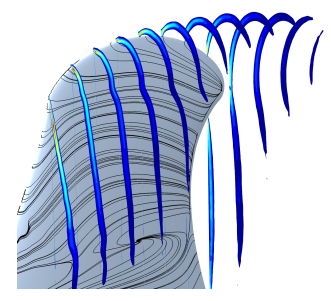

(d) $\alpha_{F}=20^{\circ}$

$|\vec{\omega}|$

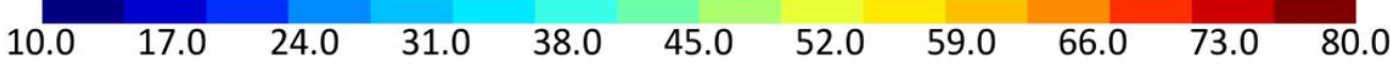

Figure B.7: Vorticity cross sections on the suction surface, $\Lambda_{t i p}=15^{\circ}$.

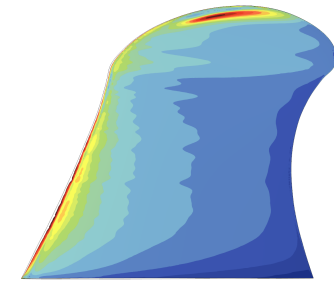

(a) $\alpha_{F}=5^{\circ}$

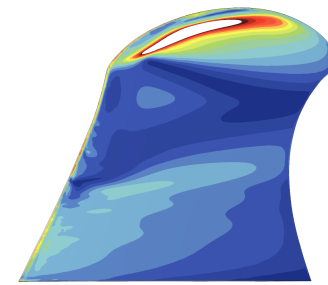

(b) $\alpha_{F}=10^{\circ}$

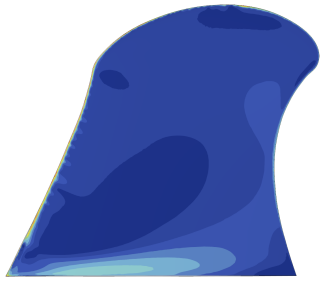

(c) $\alpha_{F}=15^{\circ}$

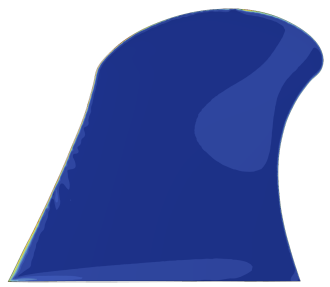

(d) $\alpha_{F}=20^{\circ}$

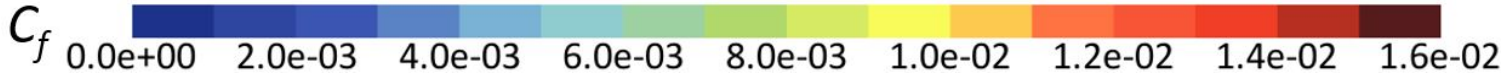

Figure B.8: $C_{f}$ coefficient contours on the suction surface, $\Lambda_{t i p}=15^{\circ}$. 
B.2.2 $\Lambda_{t i p}=20^{\circ}$
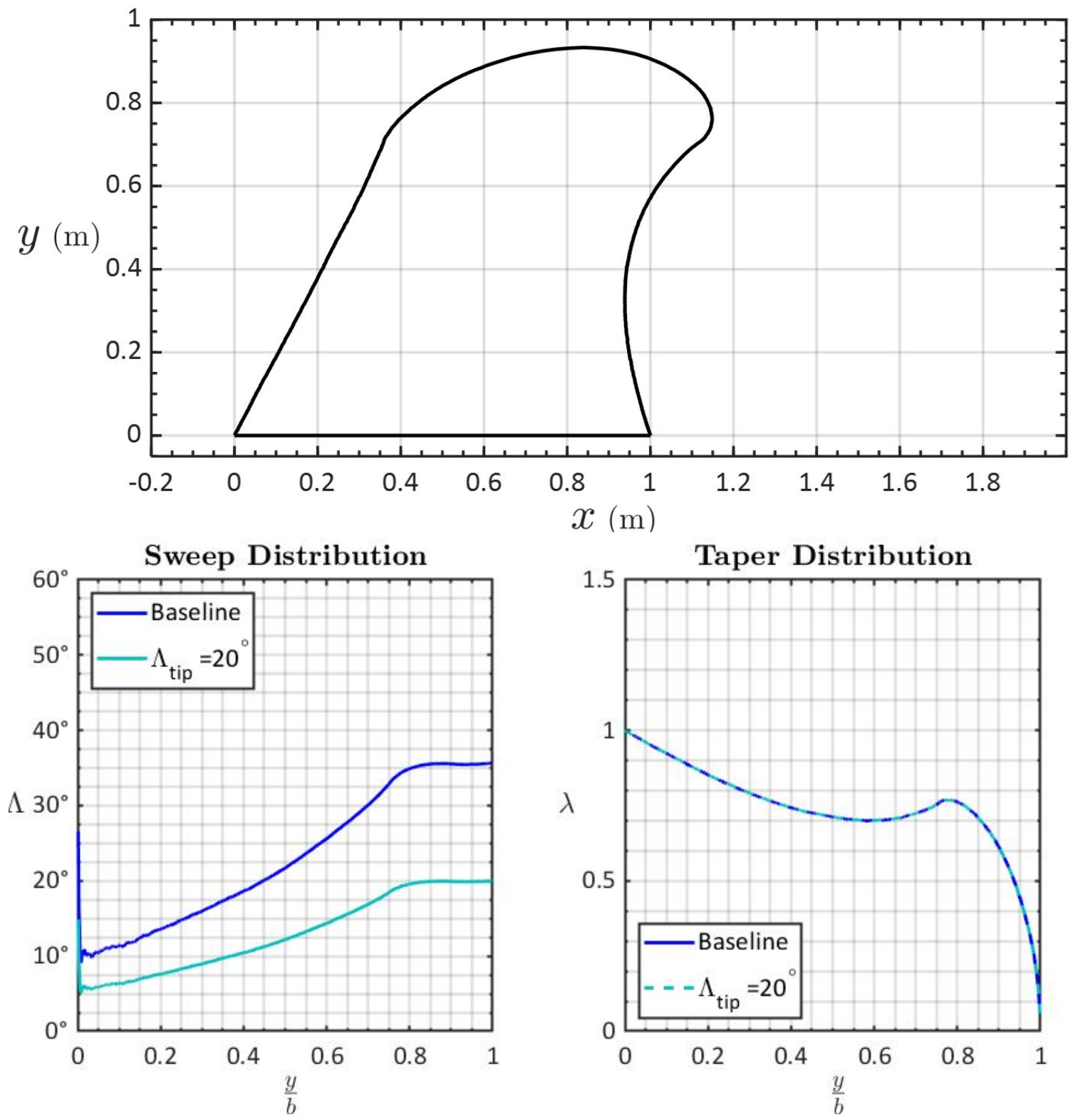

Figure B.9: Planform characteristics, $\Lambda_{t i p}=20^{\circ}$. 

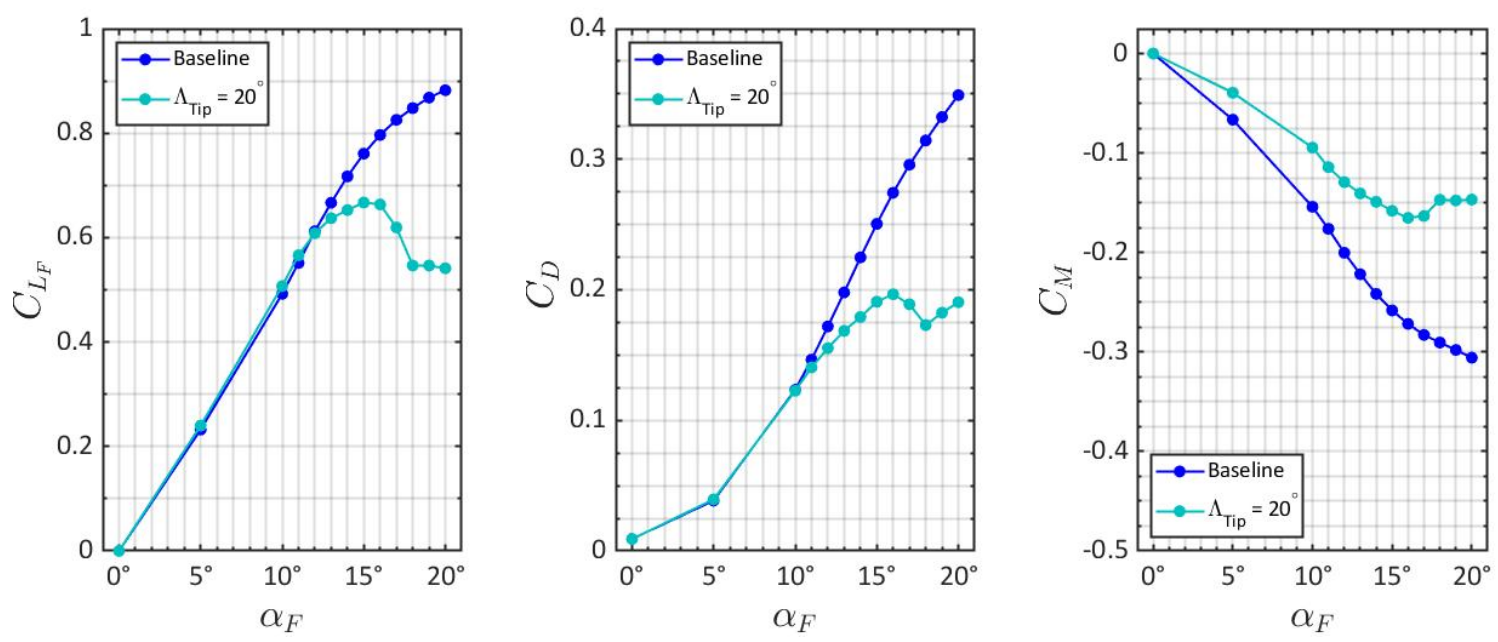

Figure B.10: Lift, drag, and moment coefficients, $\Lambda_{t i p}=20^{\circ}$.

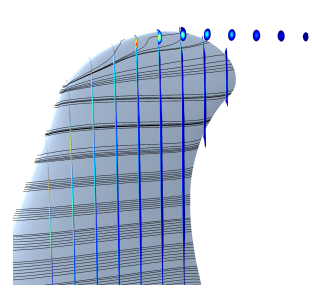

(a) $\alpha_{F}=5^{\circ}$

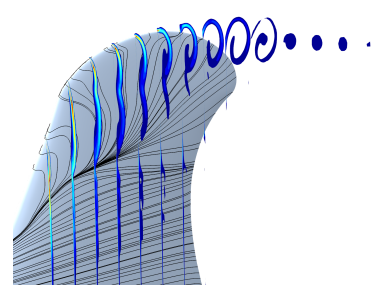

(b) $\alpha_{F}=10^{\circ}$

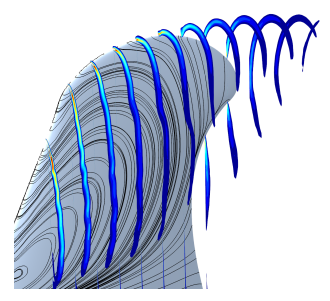

(c) $\alpha_{F}=15^{\circ}$

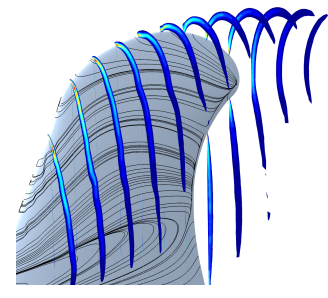

(d) $\alpha_{F}=20^{\circ}$

$|\vec{\omega}|$

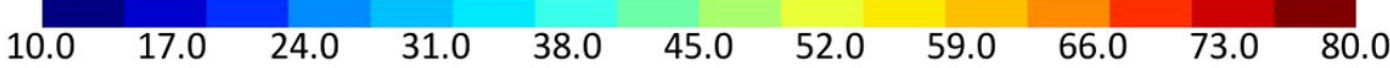

Figure B.11: Vorticity cross sections on the suction surface, $\Lambda_{t i p}=20^{\circ}$.

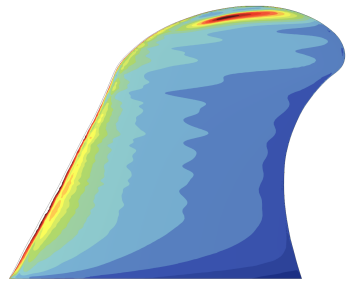

(a) $\alpha_{F}=5^{\circ}$

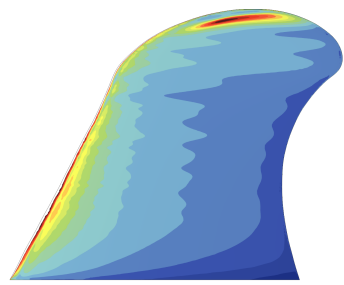

(b) $\alpha_{F}=10^{\circ}$

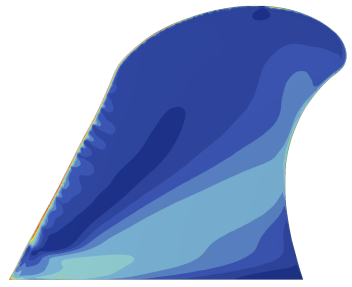

(c) $\alpha_{F}=15^{\circ}$

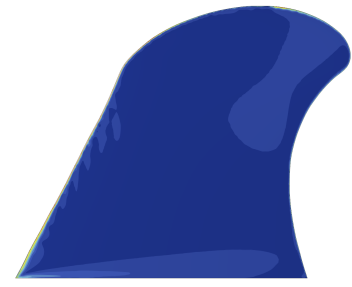

(d) $\alpha_{F}=20^{\circ}$

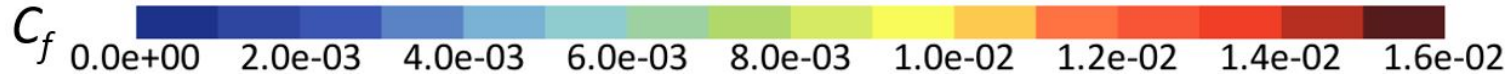

Figure B.12: $C_{f}$ coefficient contours on the suction surface, $\Lambda_{t i p}=20^{\circ}$. 
B.2.3 $\Lambda_{t i p}=25^{\circ}$
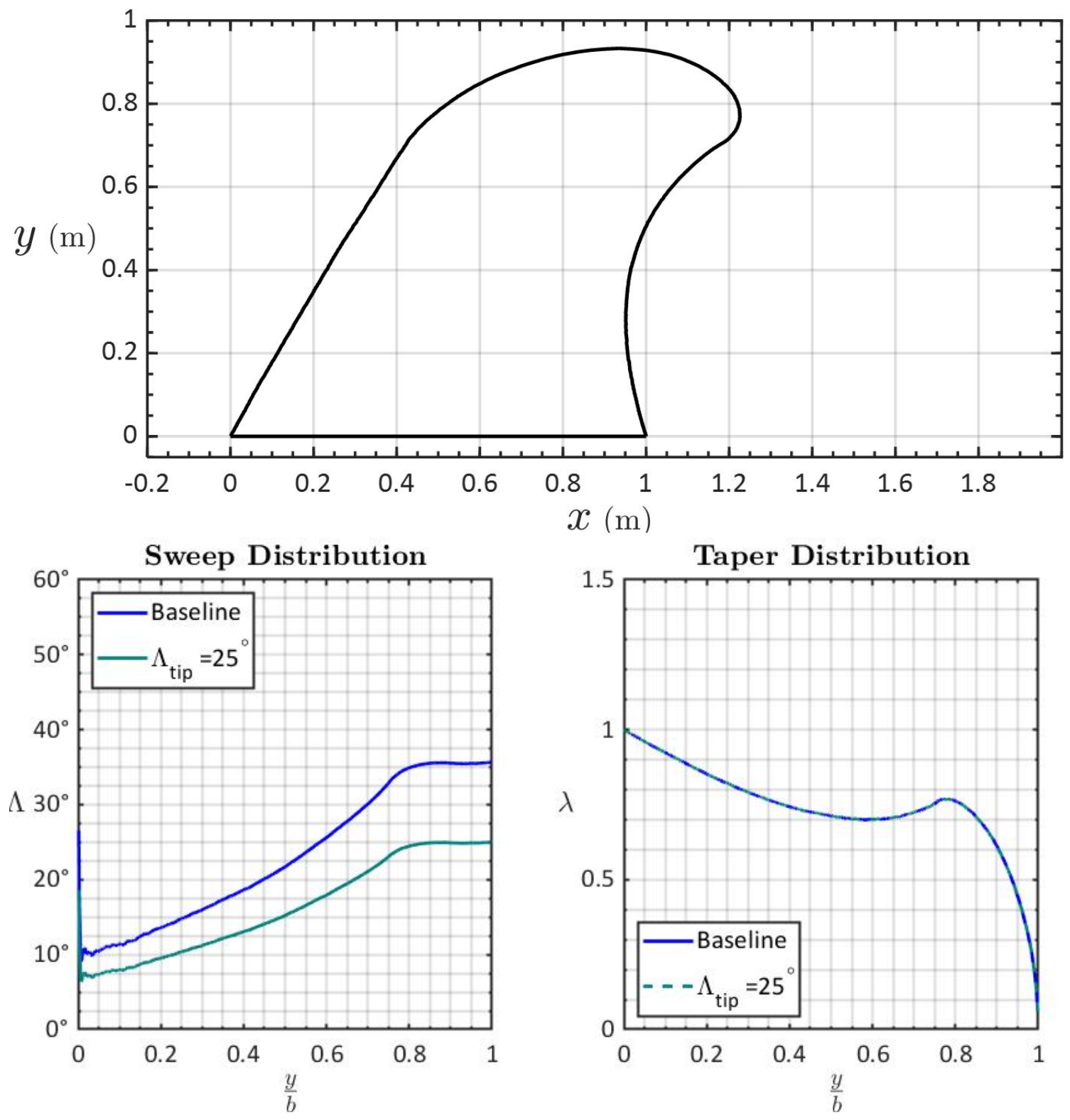

Figure B.13: Planform characteristics, $\Lambda_{t i p}=25^{\circ}$. 

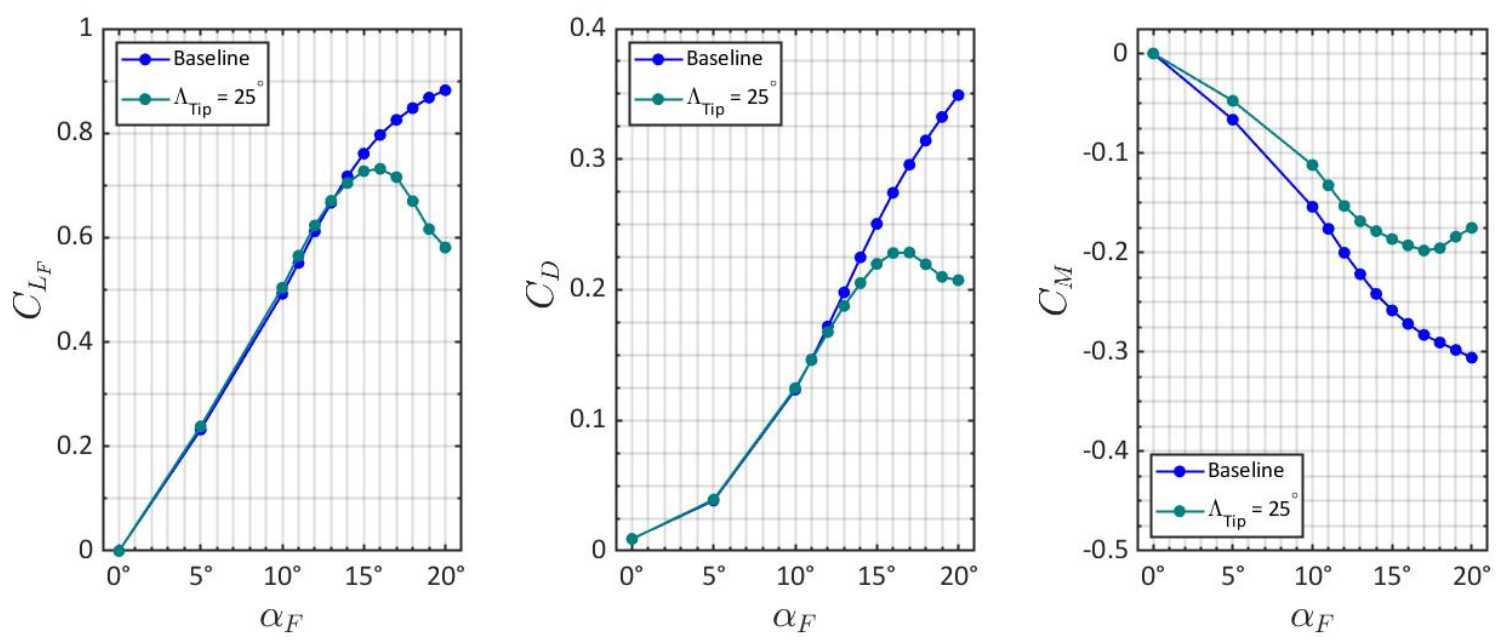

Figure B.14: Lift, drag, and moment coefficients, $\Lambda_{t i p}=25^{\circ}$.

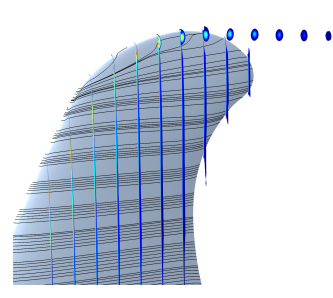

(a) $\alpha_{F}=5^{\circ}$

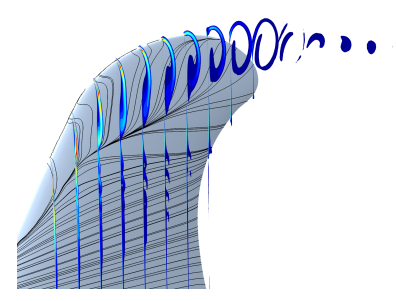

(b) $\alpha_{F}=10^{\circ}$

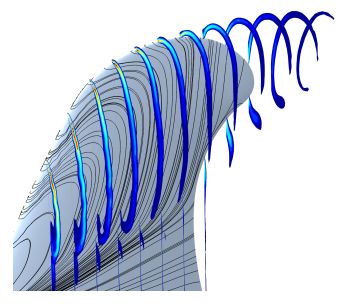

(c) $\alpha_{F}=15^{\circ}$

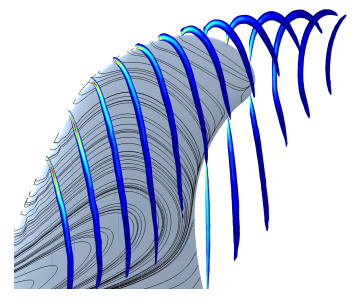

(d) $\alpha_{F}=20^{\circ}$

$|\vec{\omega}|$

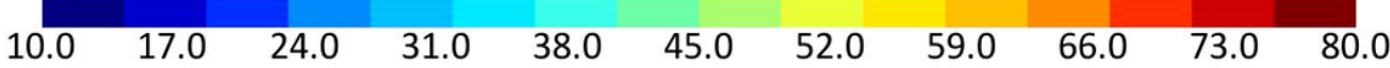

Figure B.15: Vorticity cross sections on the suction surface, $\Lambda_{t i p}=25^{\circ}$.

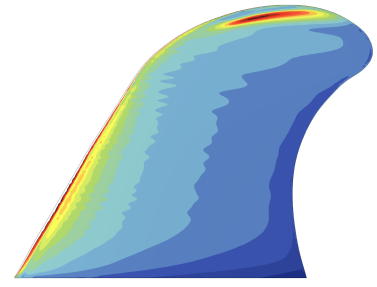

(a) $\alpha_{F}=5^{\circ}$

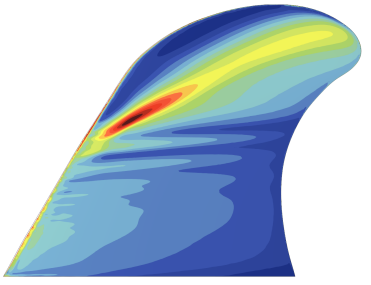

(b) $\alpha_{F}=10^{\circ}$

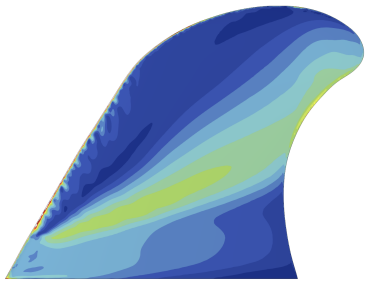

(c) $\alpha_{F}=15^{\circ}$

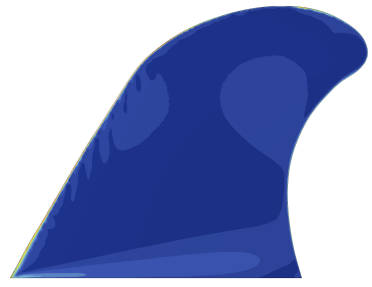

(d) $\alpha_{F}=20^{\circ}$

$\begin{array}{lllllllll}C_{f}{ }_{0.0 \mathrm{e}+00} & 2.0 \mathrm{e}-03 & 4.0 \mathrm{e}-03 & 6.0 \mathrm{e}-03 & 8.0 \mathrm{e}-03 & 1.0 \mathrm{e}-02 & 1.2 \mathrm{e}-02 & 1.4 \mathrm{e}-02 & 1.6 \mathrm{e}-02\end{array}$

Figure B.16: $C_{f}$ coefficient contours on the suction surface, $\Lambda_{t i p}=25^{\circ}$. 
B.2.4 $\Lambda_{t i p}=30^{\circ}$
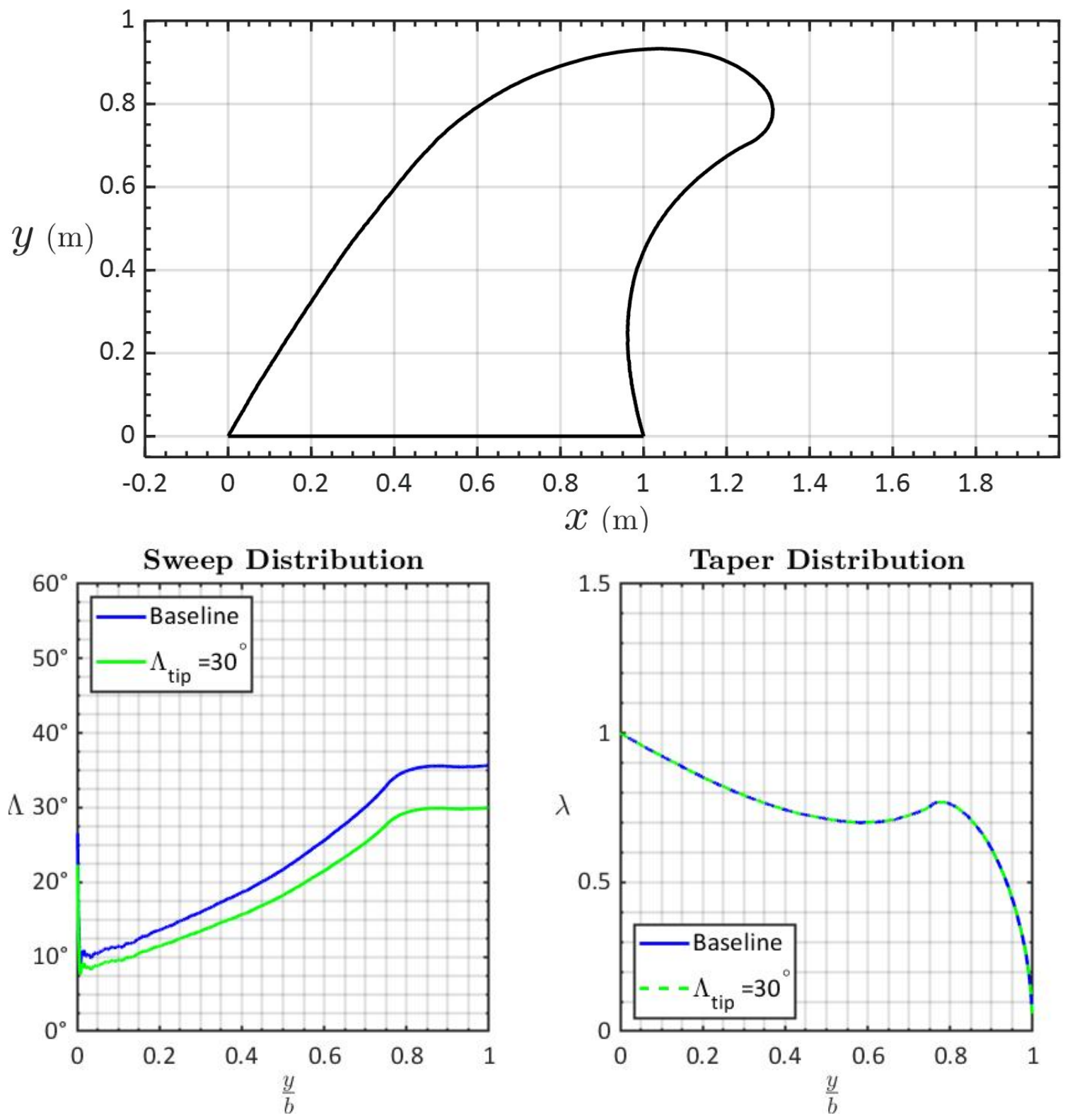

Figure B.17: Planform characteristics, $\Lambda_{t i p}=30^{\circ}$. 

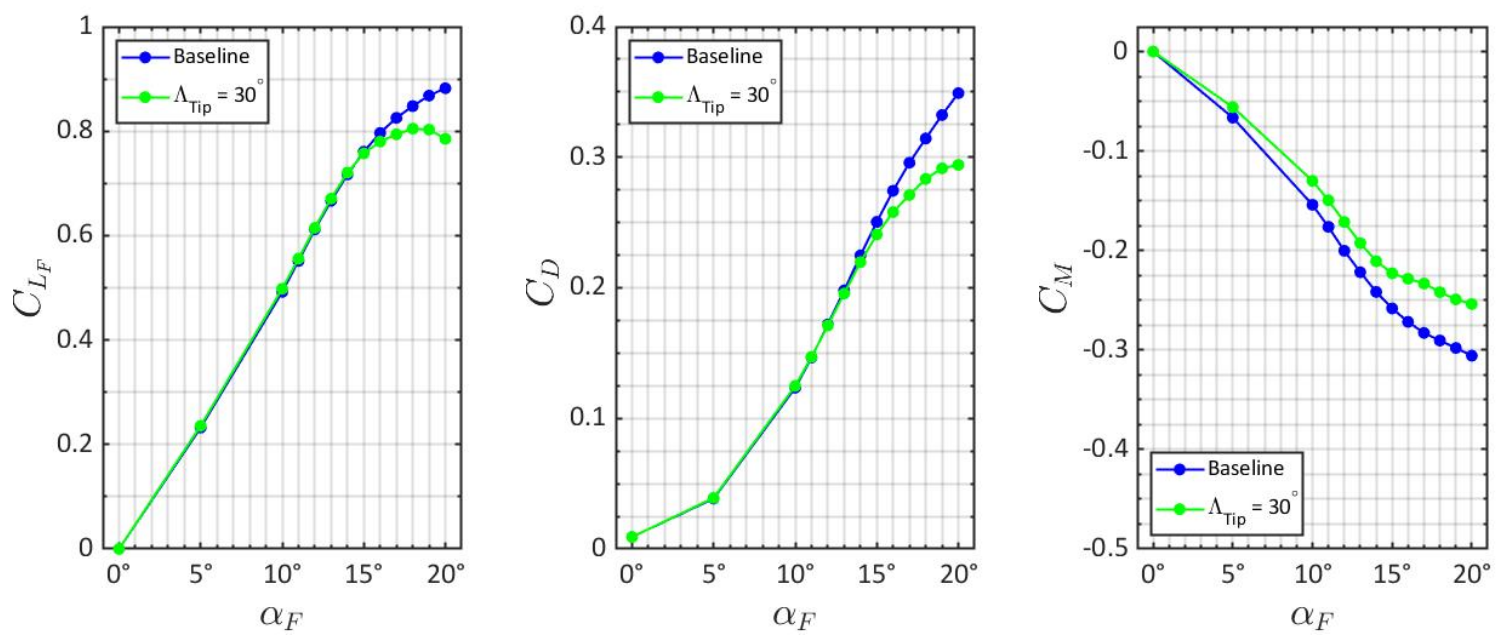

Figure B.18: Lift, drag, and moment coefficients, $\Lambda_{t i p}=30^{\circ}$.

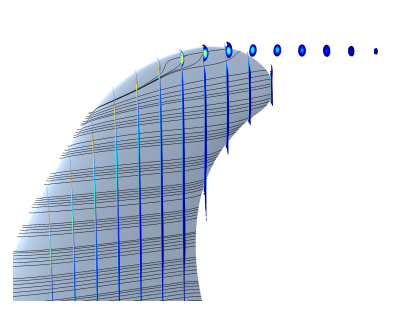

(a) $\alpha_{F}=5^{\circ}$

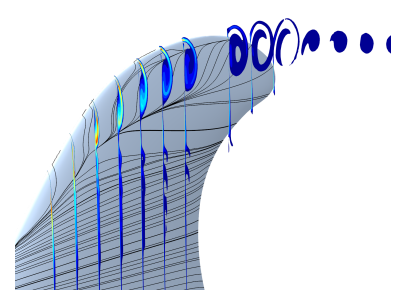

(b) $\alpha_{F}=10^{\circ}$

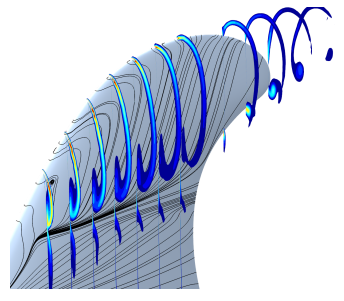

(c) $\alpha_{F}=15^{\circ}$

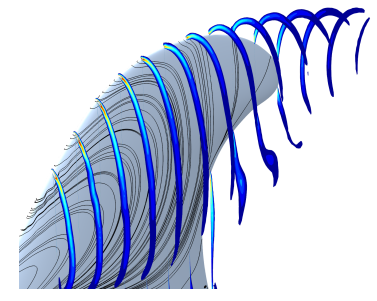

(d) $\alpha_{F}=20^{\circ}$

$|\vec{\omega}|$

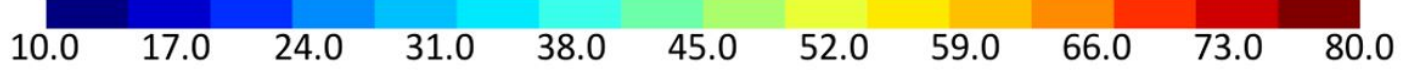

Figure B.19: Vorticity cross sections on the suction surface, $\Lambda_{t i p}=30^{\circ}$.

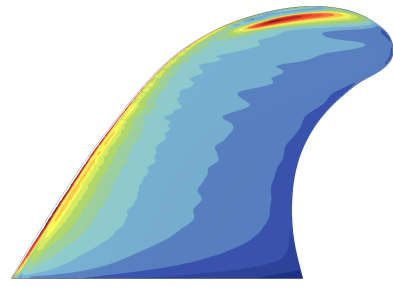

(a) $\alpha_{F}=5^{\circ}$

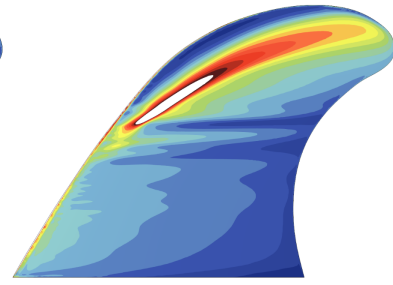

(b) $\alpha_{F}=10^{\circ}$

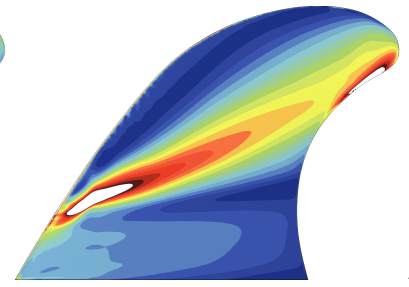

(c) $\alpha_{F}=15^{\circ}$

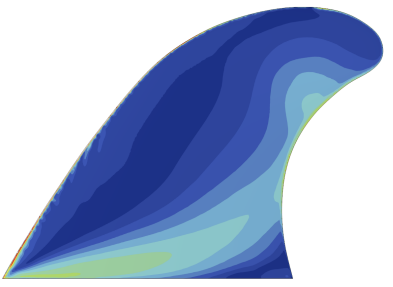

(d) $\alpha_{F}=20^{\circ}$

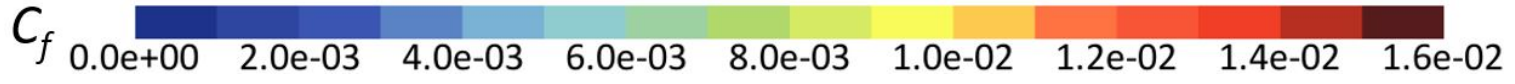

Figure B.20: $C_{f}$ coefficient contours on the suction surface, $\Lambda_{t i p}=30^{\circ}$. 
B.2.5 $\Lambda_{t i p}=40^{\circ}$
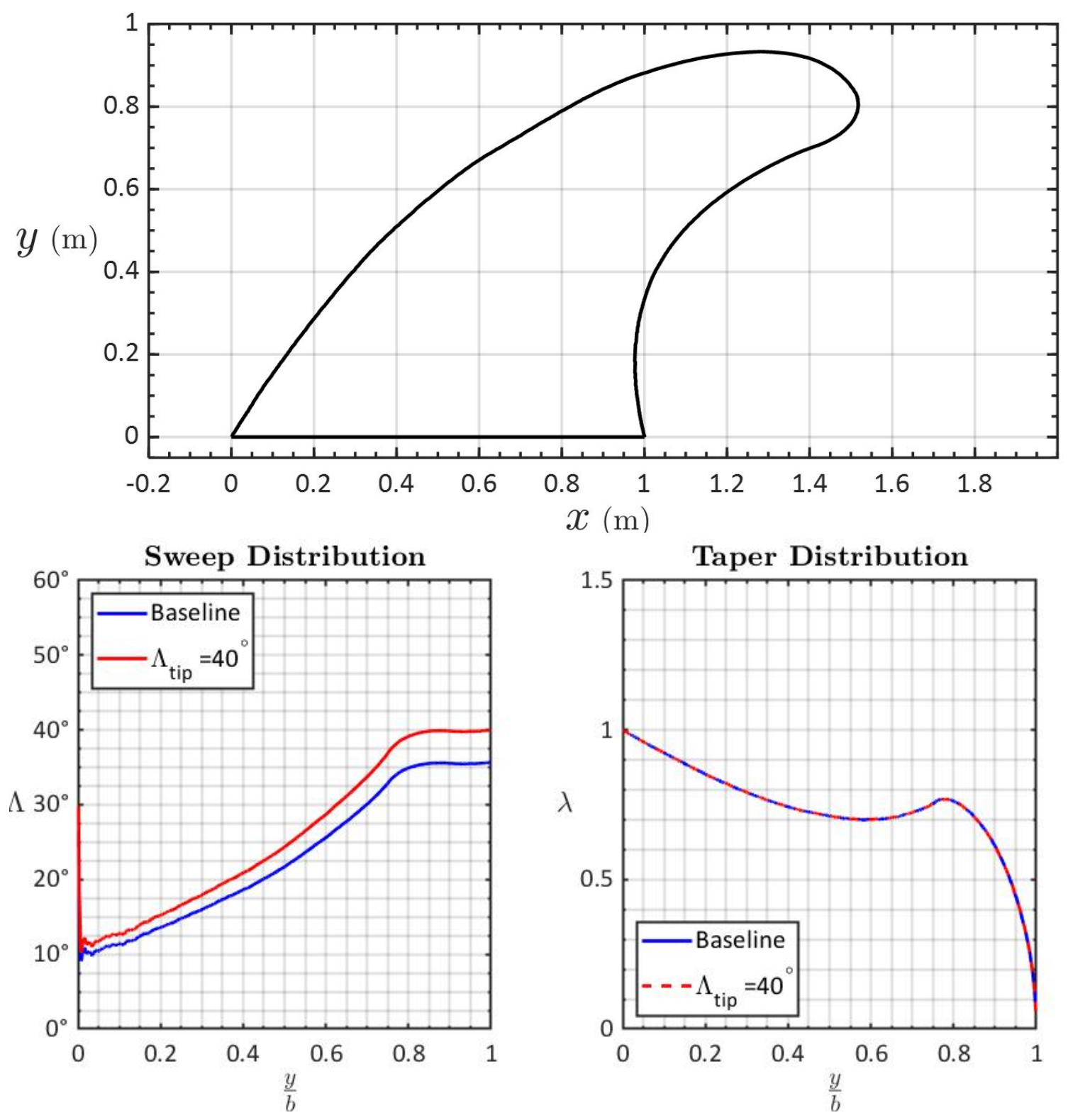

Figure B.21: Planform characteristics, $\Lambda_{t i p}=40^{\circ}$. 

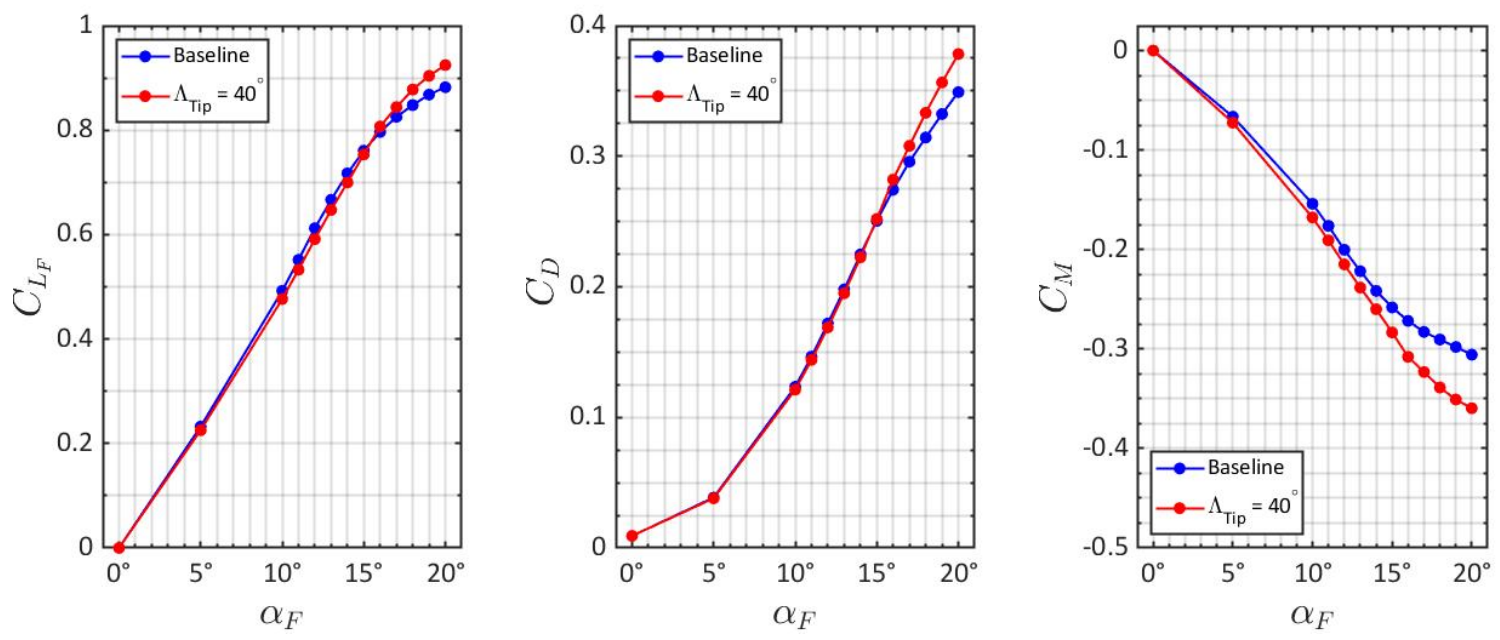

Figure B.22: Lift, drag, and moment coefficients, $\Lambda_{t i p}=40^{\circ}$.

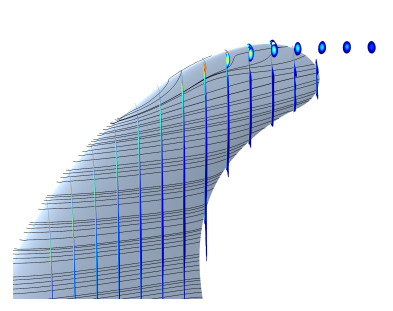

(a) $\alpha_{F}=5^{\circ}$

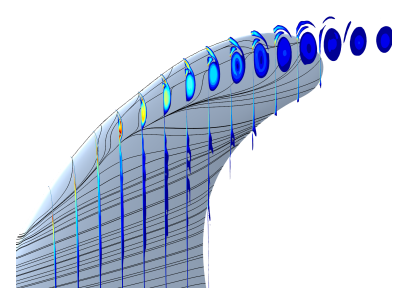

(b) $\alpha_{F}=10^{\circ}$

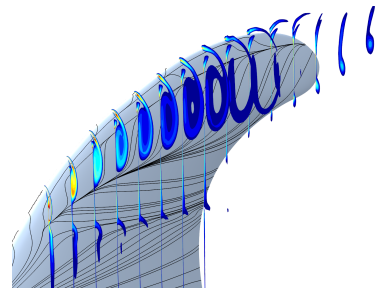

(c) $\alpha_{F}=15^{\circ}$

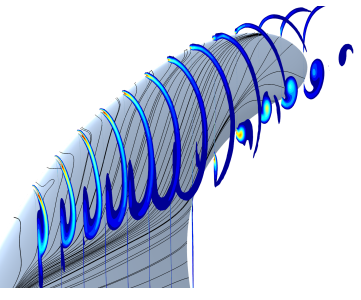

(d) $\alpha_{F}=20^{\circ}$

$|\vec{\omega}|$

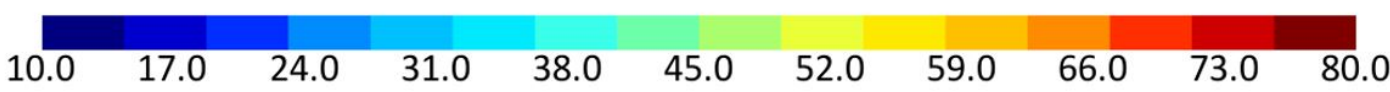

Figure B.23: Vorticity cross sections on the suction surface, $\Lambda_{t i p}=40^{\circ}$.

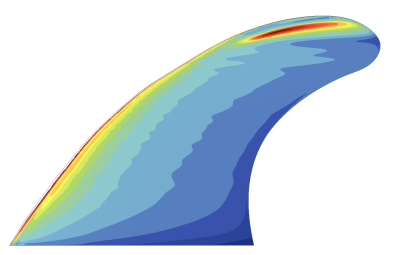

(a) $\alpha_{F}=5^{\circ}$

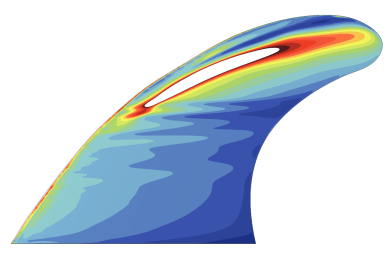

(b) $\alpha_{F}=10^{\circ}$

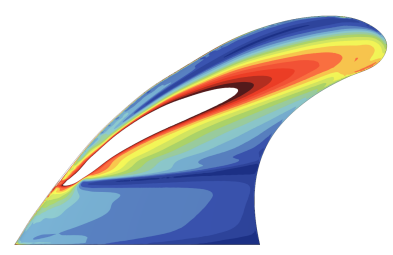

(c) $\alpha_{F}=15^{\circ}$

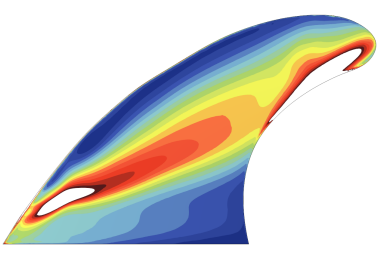

(d) $\alpha_{F}=20^{\circ}$

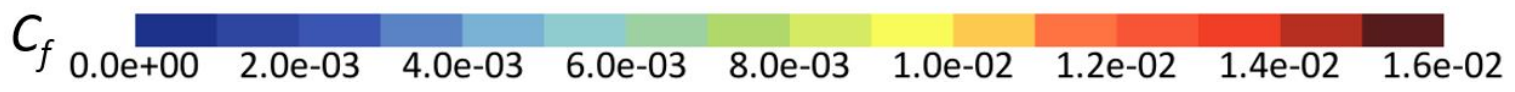

Figure B.24: $C_{f}$ coefficient contours on the suction surface, $\Lambda_{t i p}=40^{\circ}$. 
B.2.6 $\Lambda_{t i p}=45^{\circ}$
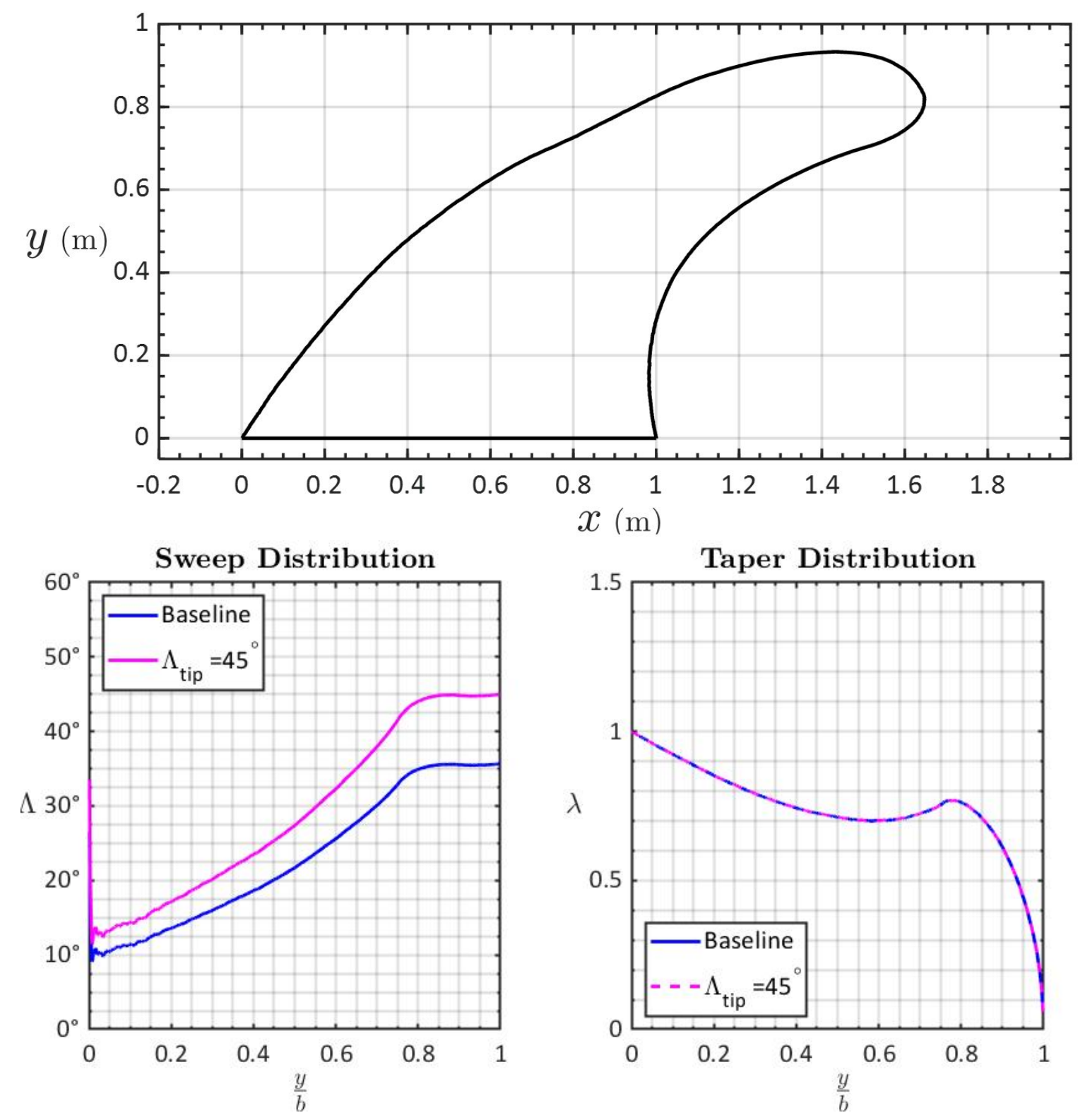

Figure B.25: Planform characteristics, $\Lambda_{t i p}=45^{\circ}$. 

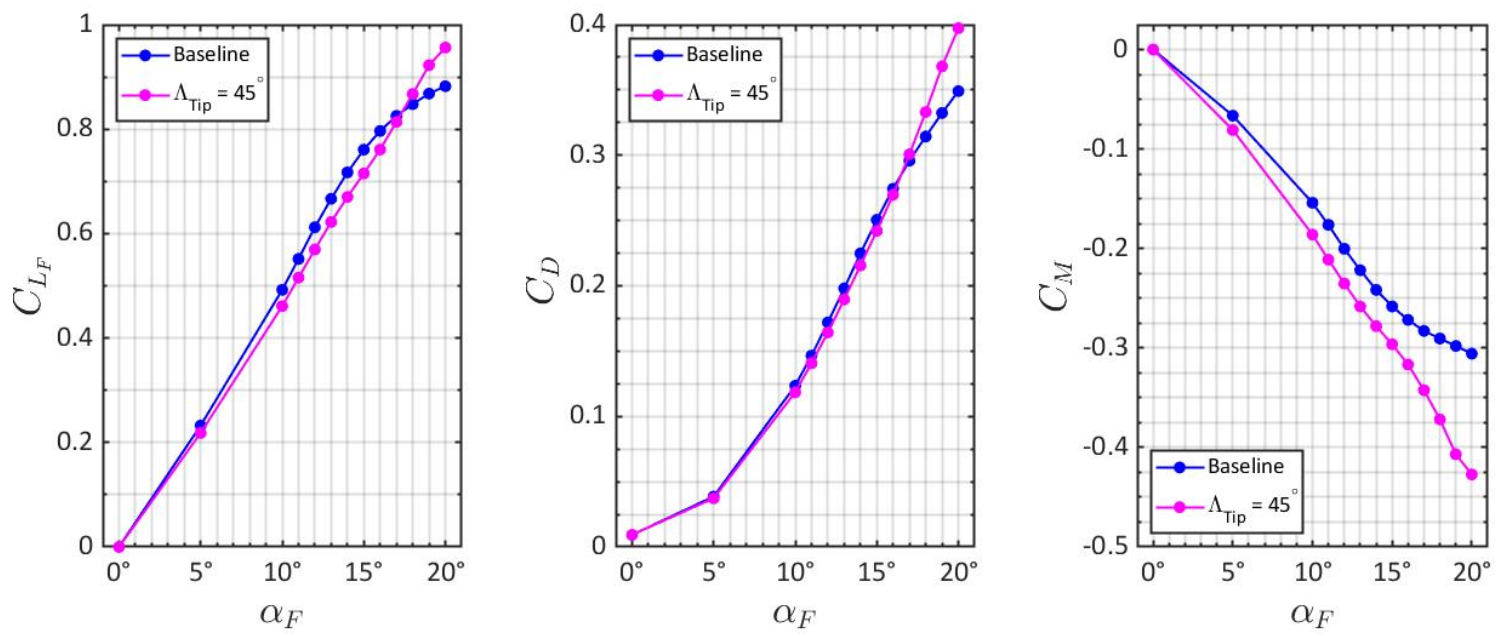

Figure B.26: Lift, drag, and moment coefficients, $\Lambda_{t i p}=45^{\circ}$.

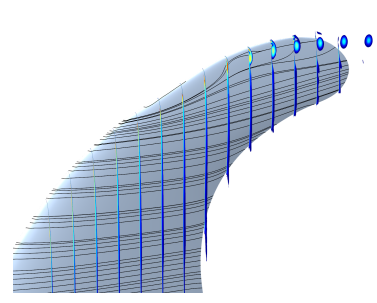

(a) $\alpha_{F}=5^{\circ}$

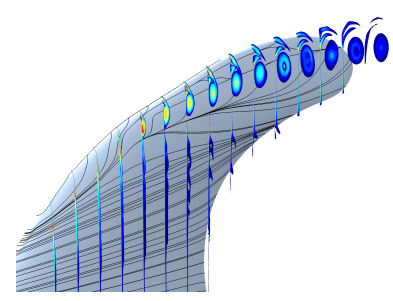

(b) $\alpha_{F}=10^{\circ}$

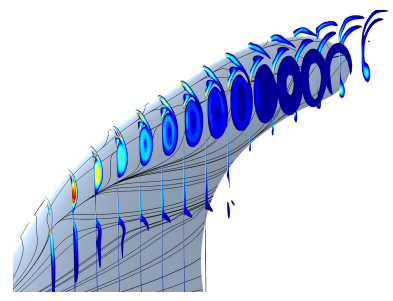

(c) $\alpha_{F}=15^{\circ}$

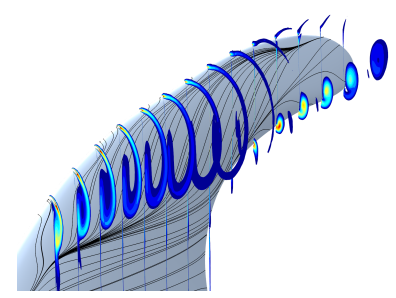

(d) $\alpha_{F}=20^{\circ}$

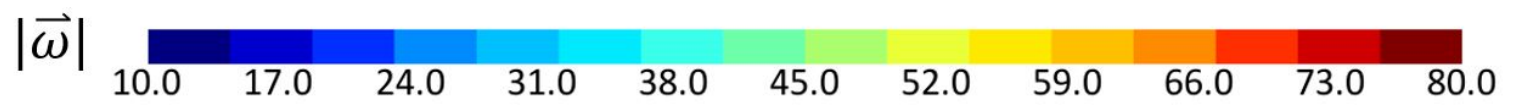

Figure B.27: Vorticity cross sections on the suction surface, $\Lambda_{t i p}=45^{\circ}$.

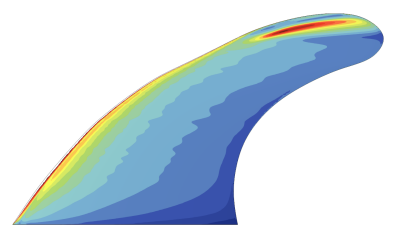

(a) $\alpha_{F}=5^{\circ}$

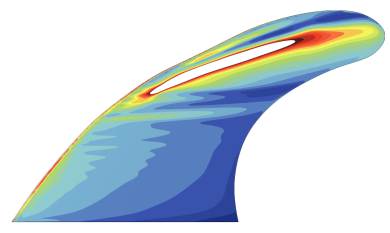

(b) $\alpha_{F}=10^{\circ}$

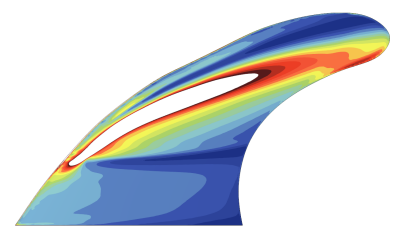

(c) $\alpha_{F}=15^{\circ}$

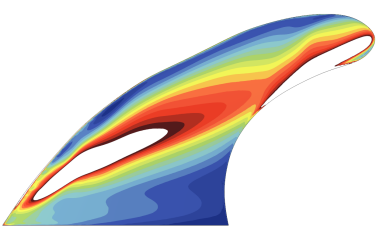

(d) $\alpha_{F}=20^{\circ}$

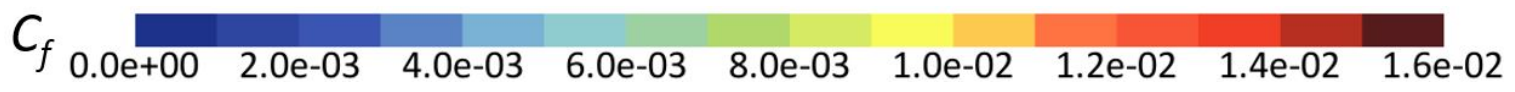

Figure B.28: $C_{f}$ coefficient contours on the suction surface, $\Lambda_{t i p}=45^{\circ}$. 
B.2.7 $\Lambda_{t i p}=50^{\circ}$
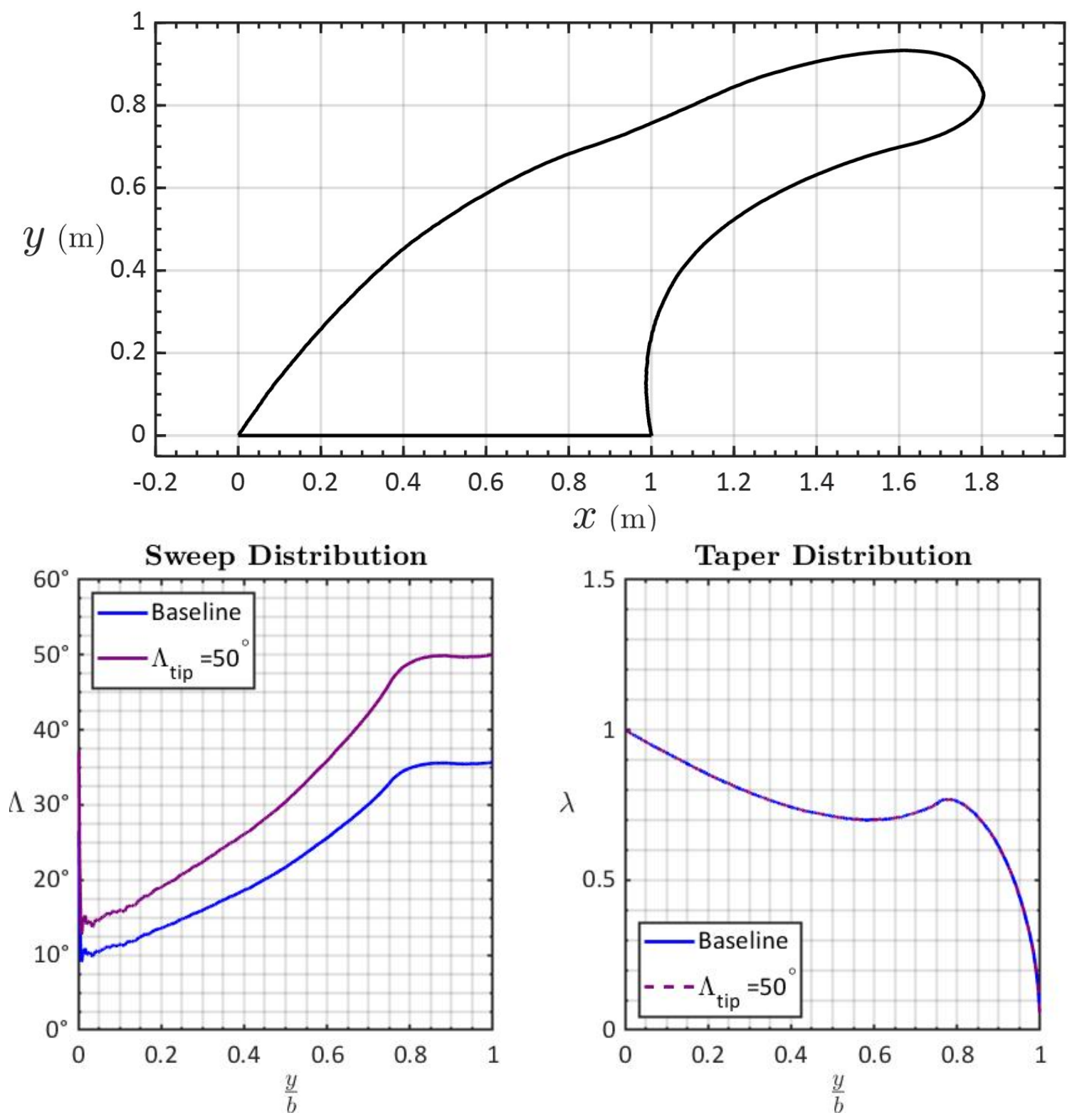

Figure B.29: Planform characteristics, $\Lambda_{t i p}=50^{\circ}$. 

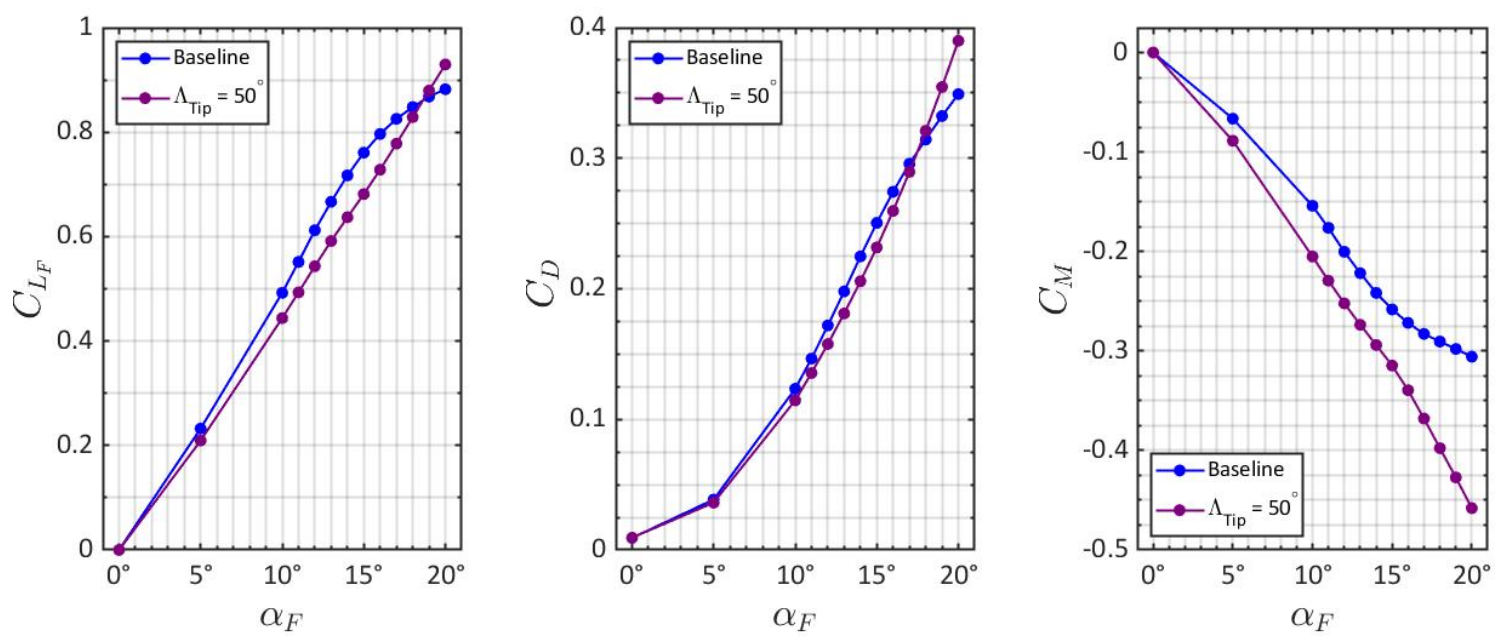

Figure B.30: Lift, drag, and moment coefficients, $\Lambda_{t i p}=50^{\circ}$.

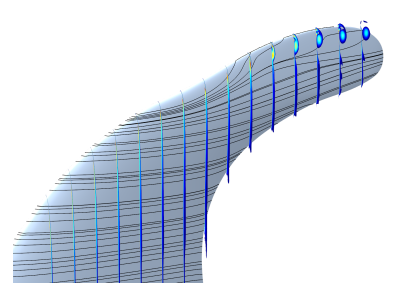

(a) $\alpha_{F}=5^{\circ}$

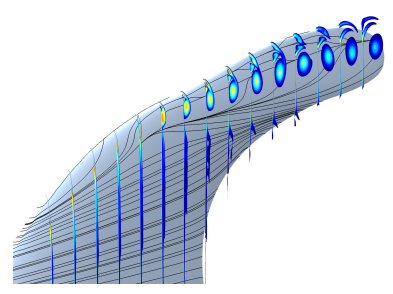

(b) $\alpha_{F}=10^{\circ}$

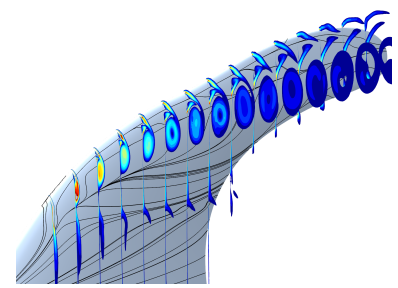

(c) $\alpha_{F}=15^{\circ}$

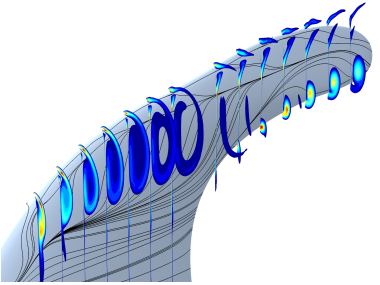

(d) $\alpha_{F}=20^{\circ}$

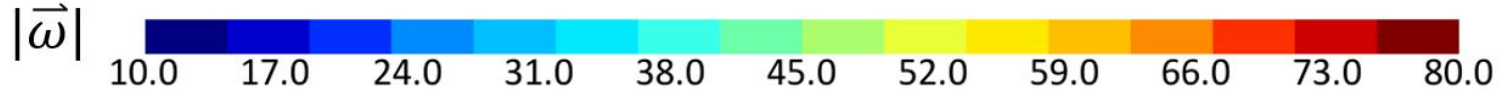

Figure B.31: Vorticity cross sections on the suction surface, $\Lambda_{t i p}=50^{\circ}$.

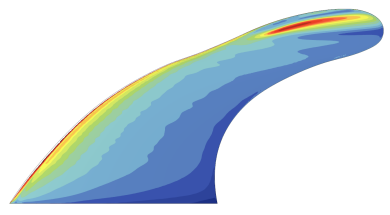

(a) $\alpha_{F}=5^{\circ}$

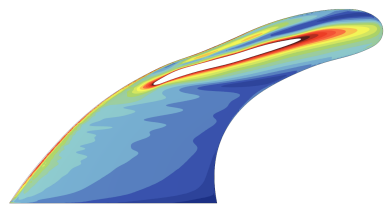

(b) $\alpha_{F}=10^{\circ}$

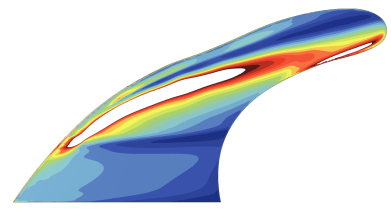

(c) $\alpha_{F}=15^{\circ}$

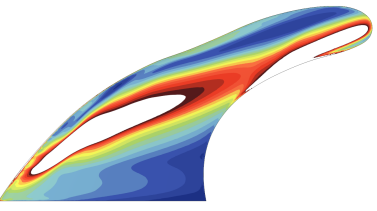

(d) $\alpha_{F}=20^{\circ}$

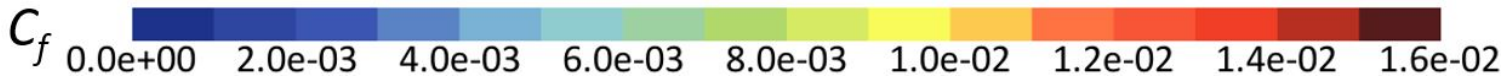

Figure B.32: $C_{f}$ coefficient contours on the suction surface, $\Lambda_{t i p}=50^{\circ}$. 
B.2.8 $\Lambda_{t i p}=55^{\circ}$
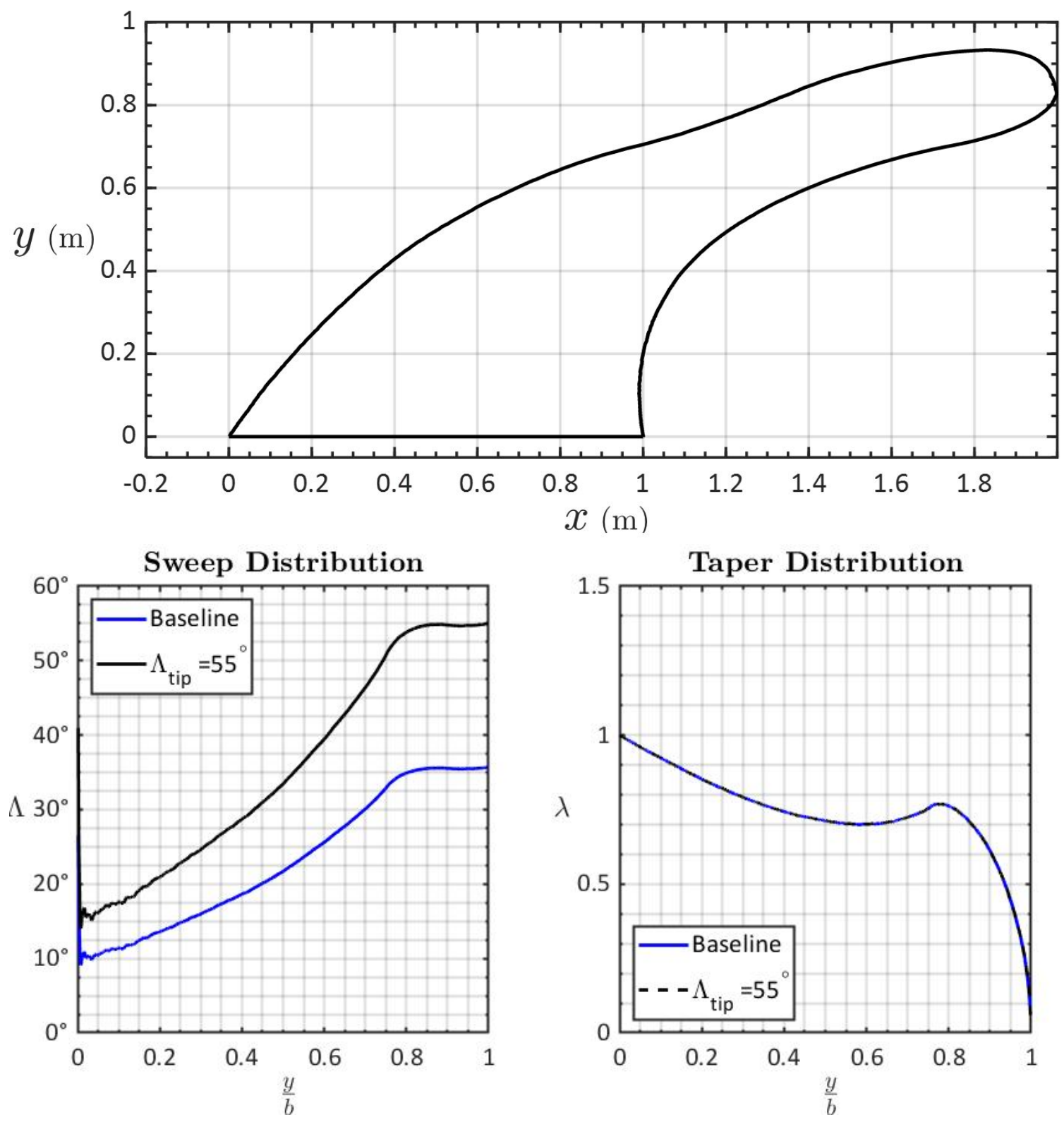

Figure B.33: Planform characteristics, $\Lambda_{t i p}=55^{\circ}$. 

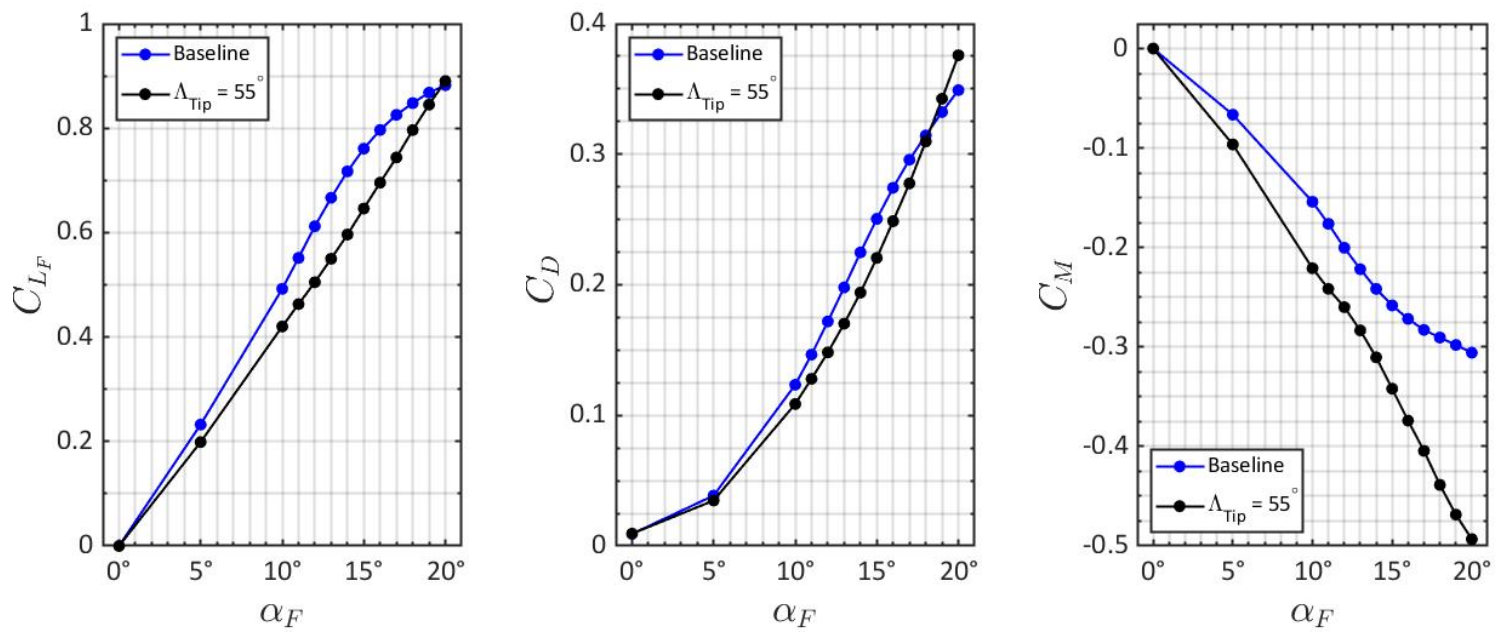

Figure B.34: Lift, drag, and moment coefficients, $\Lambda_{t i p}=55^{\circ}$.

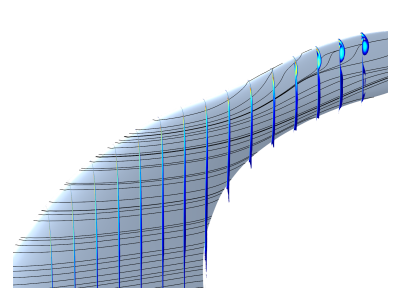

(a) $\alpha_{F}=5^{\circ}$

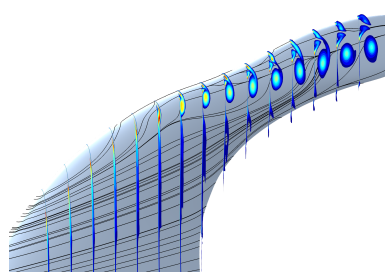

(b) $\alpha_{F}=10^{\circ}$

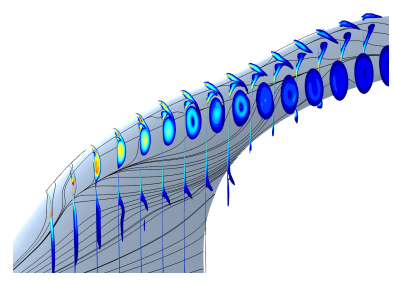

(c) $\alpha_{F}=15^{\circ}$

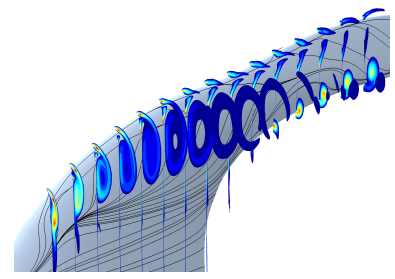

(d) $\alpha_{F}=20^{\circ}$

$|\vec{\omega}|$ $\begin{array}{lllllllllll}10.0 & 17.0 & 24.0 & 31.0 & 38.0 & 45.0 & 52.0 & 59.0 & 66.0 & 73.0 & 80.0\end{array}$

Figure B.35: Vorticity cross sections on the suction surface, $\Lambda_{t i p}=55^{\circ}$.

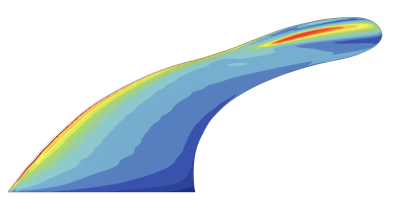

(a) $\alpha_{F}=5^{\circ}$

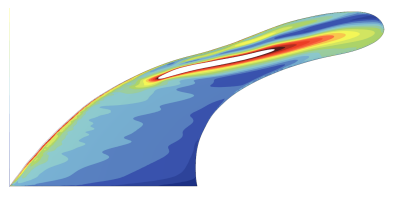

(b) $\alpha_{F}=10^{\circ}$

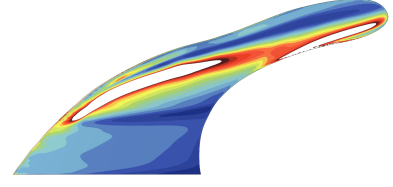

(c) $\alpha_{F}=15^{\circ}$

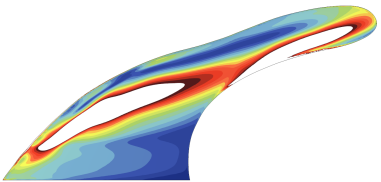

(d) $\alpha_{F}=20^{\circ}$

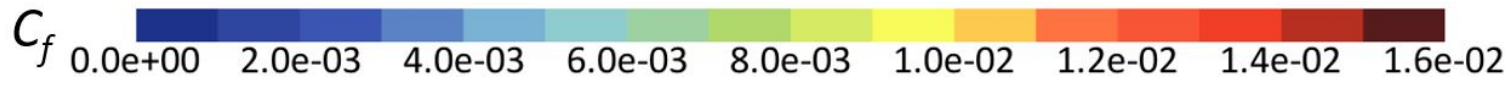

Figure B.36: $C_{f}$ coefficient contours on the suction surface, $\Lambda_{t i p}=55^{\circ}$. 


\section{B.3 Taper}

B.3.1 $K_{\text {taper }}=0.50$
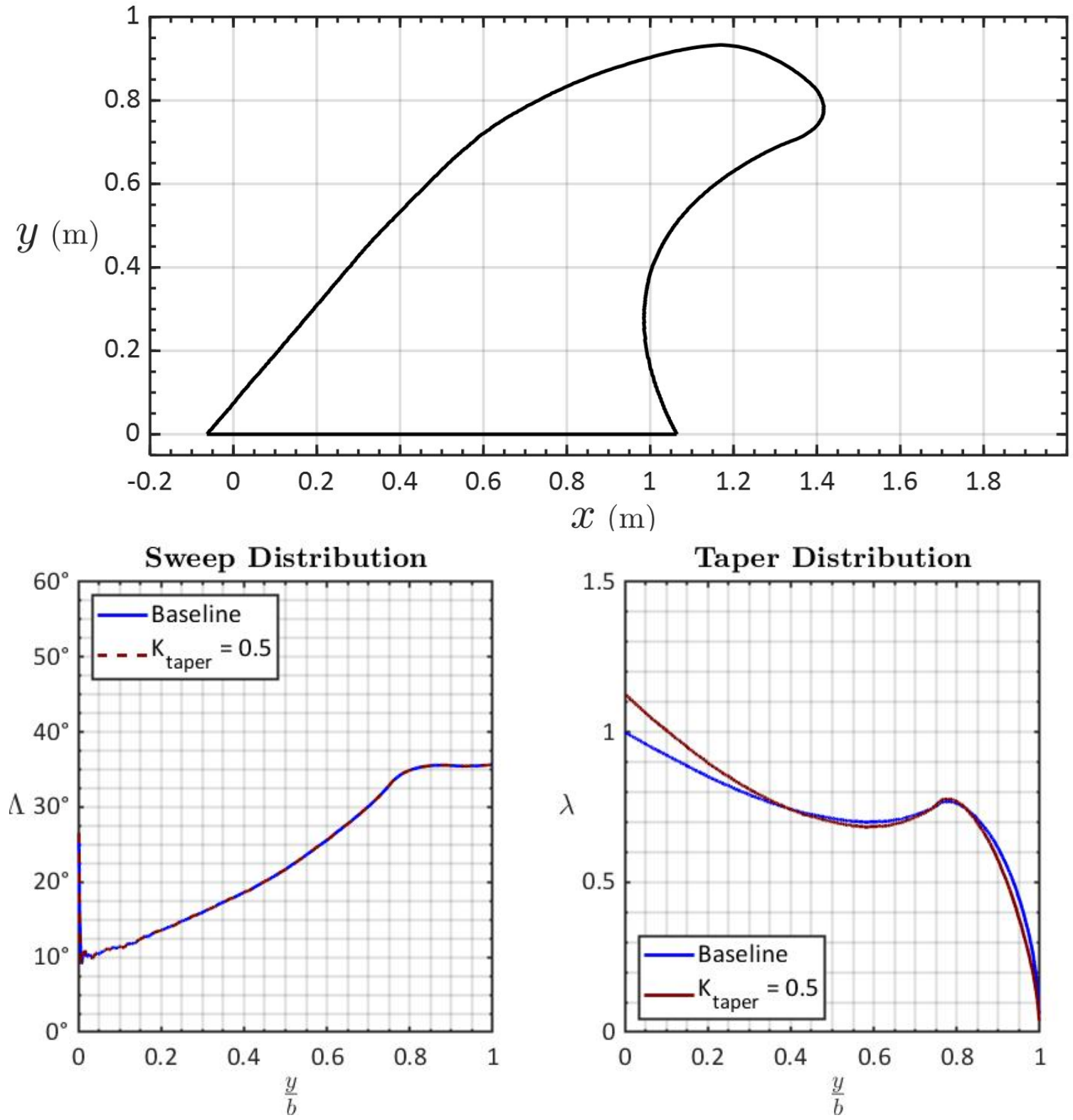

Figure B.37: Planform characteristics, $K_{\text {taper }}=0.50$. 

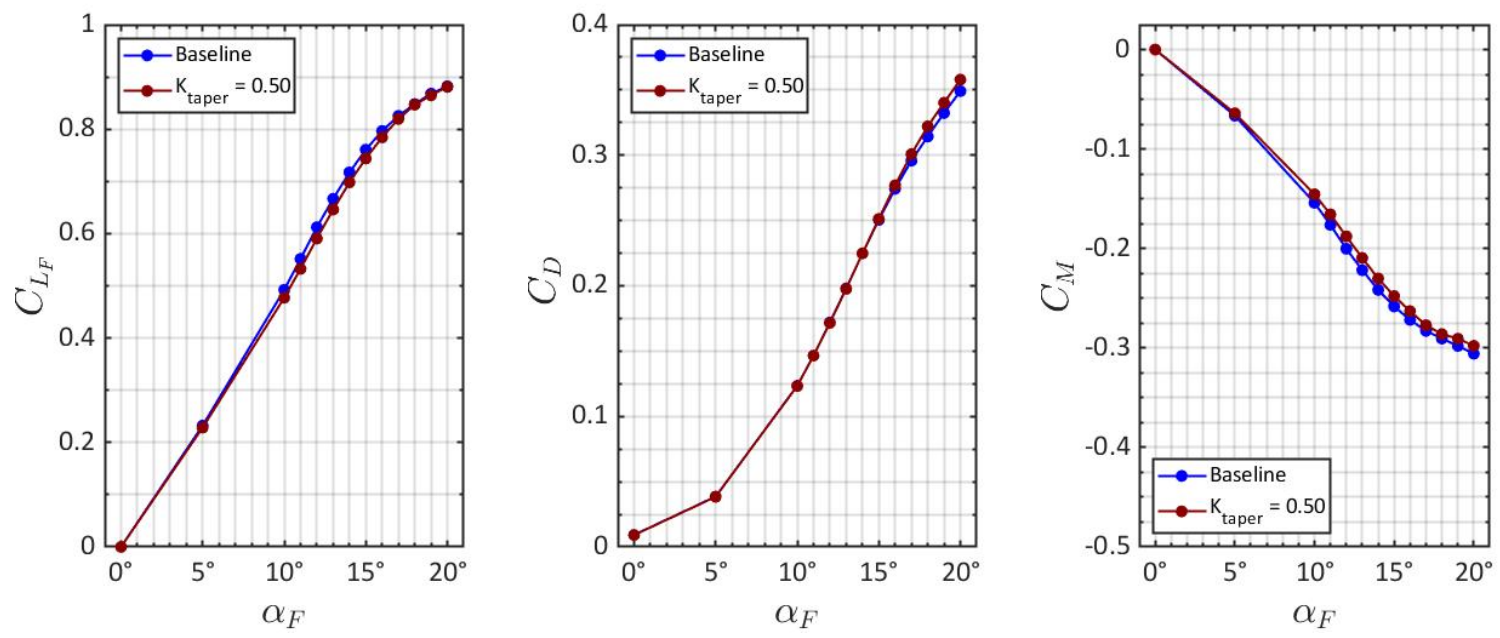

Figure B.38: Lift, drag, and moment coefficients, $K_{\text {taper }}=0.50$.

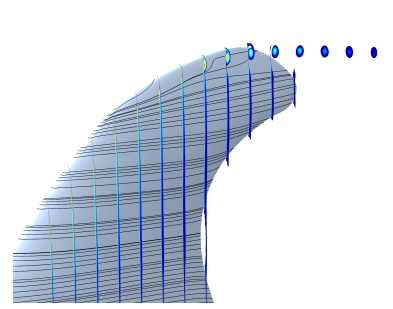

(a) $\alpha_{F}=5^{\circ}$

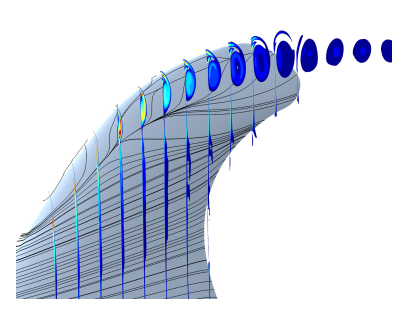

(b) $\alpha_{F}=10^{\circ}$

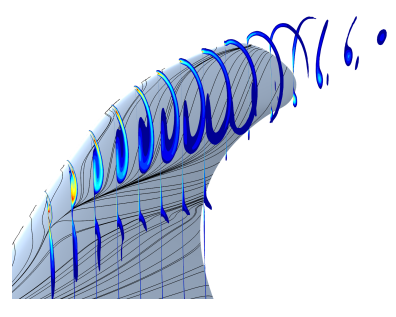

(c) $\alpha_{F}=15^{\circ}$

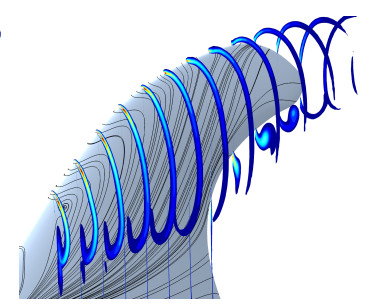

(d) $\alpha_{F}=20^{\circ}$

$|\vec{\omega}|$

$\begin{array}{lllllllllll}10.0 & 17.0 & 24.0 & 31.0 & 38.0 & 45.0 & 52.0 & 59.0 & 66.0 & 73.0 & 80.0\end{array}$

Figure B.39: Vorticity cross sections on the suction surface, $K_{\text {taper }}=0.50$.

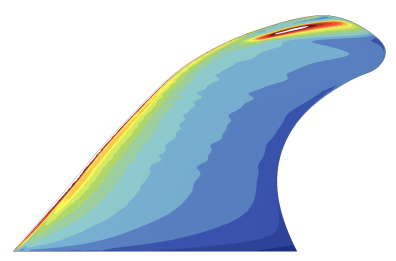

(a) $\alpha_{F}=5^{\circ}$

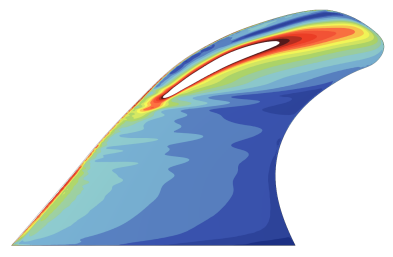

(b) $\alpha_{F}=10^{\circ}$

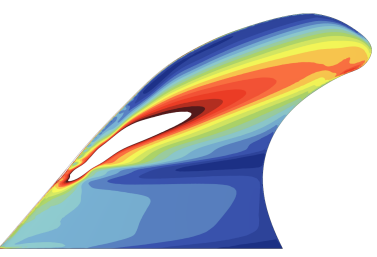

(c) $\alpha_{F}=15^{\circ}$

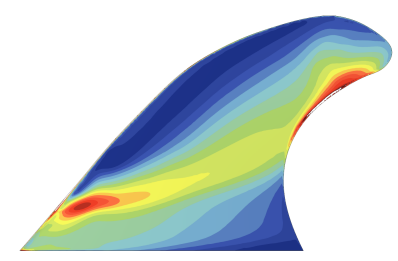

(d) $\alpha_{F}=20^{\circ}$

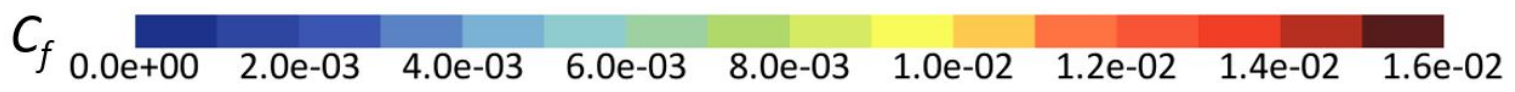

Figure B.40: $C_{f}$ coefficient contours on the suction surface, $K_{\text {taper }}=0.50$. 
B.3.2 $K_{\text {taper }}=0.75$
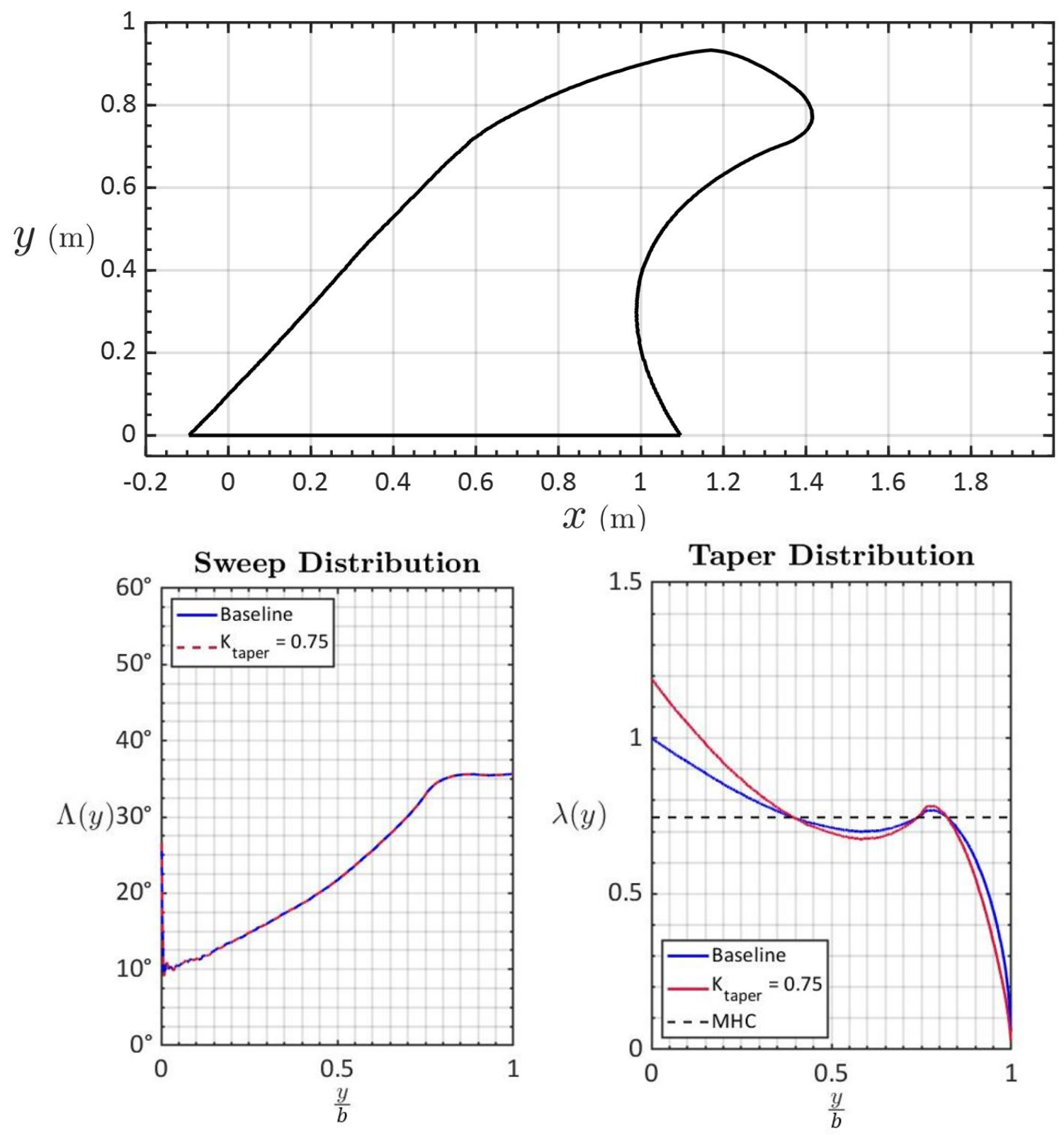

Figure B.41: Planform characteristics, $K_{\text {taper }}=0.75$. 

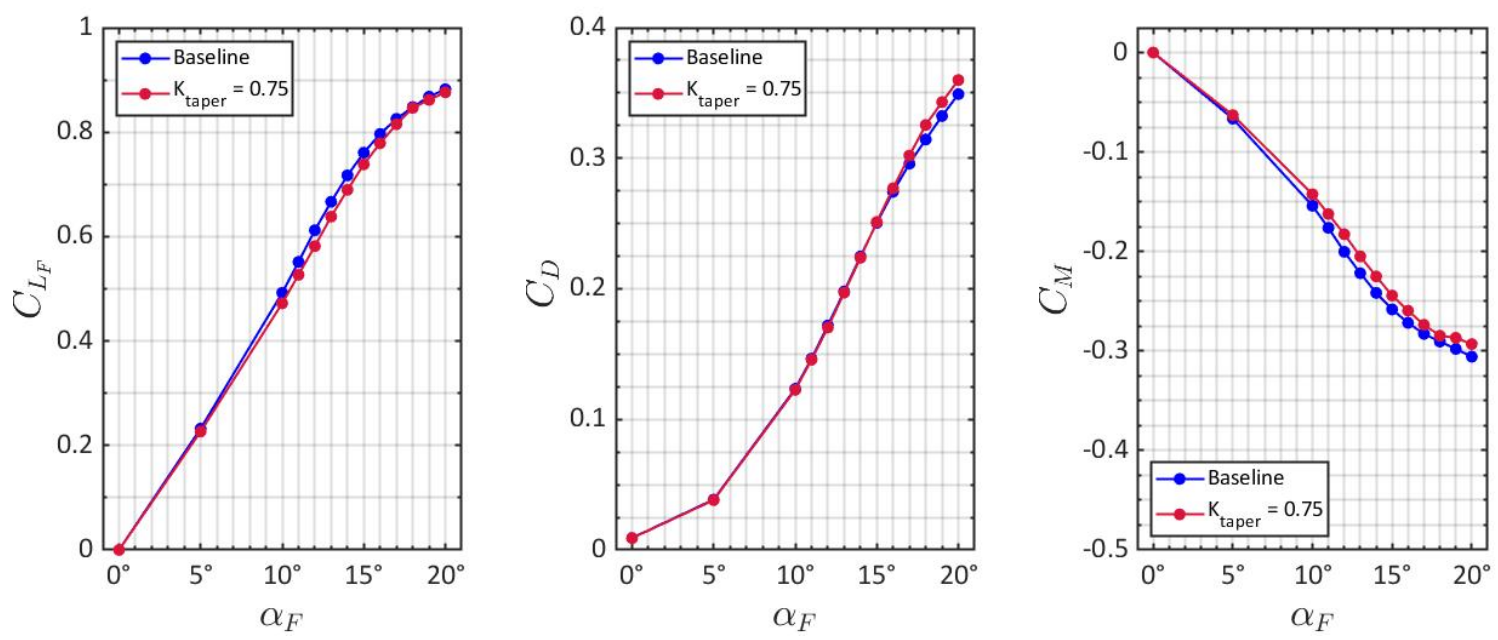

Figure B.42: Lift, drag, and moment coefficients, $K_{\text {taper }}=0.75$.

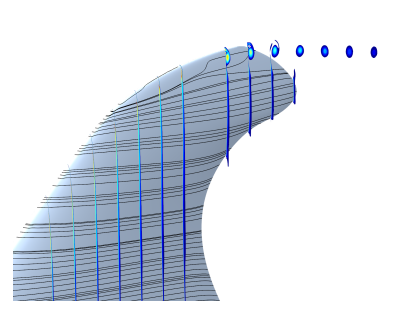

(a) $\alpha_{F}=5^{\circ}$

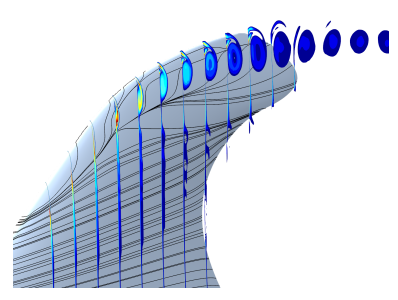

(b) $\alpha_{F}=10^{\circ}$

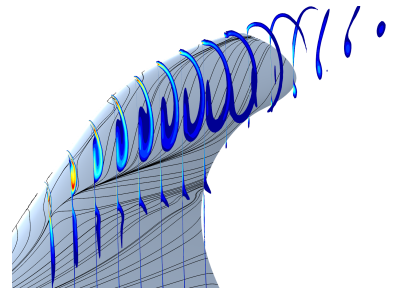

(c) $\alpha_{F}=15^{\circ}$

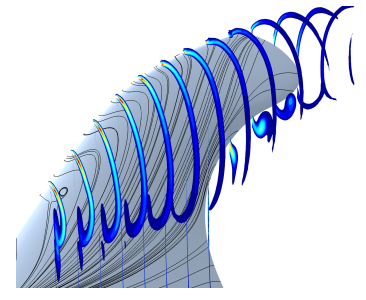

(d) $\alpha_{F}=20^{\circ}$

$|\vec{\omega}|_{10.0} \begin{array}{rrrrrrrrrr}17.0 & 24.0 & 31.0 & 38.0 & 45.0 & 52.0 & 59.0 & 66.0 & 73.0 & 80.0\end{array}$

Figure B.43: Vorticity cross sections on the suction surface, $K_{\text {taper }}=0.75$.

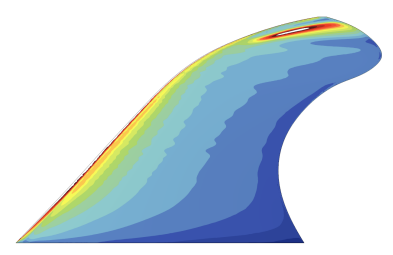

(a) $\alpha_{F}=5^{\circ}$

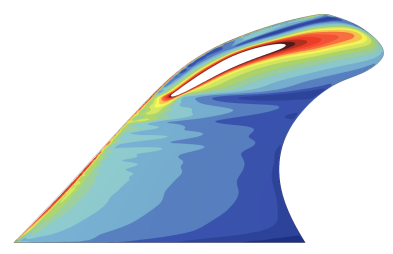

(b) $\alpha_{F}=10^{\circ}$

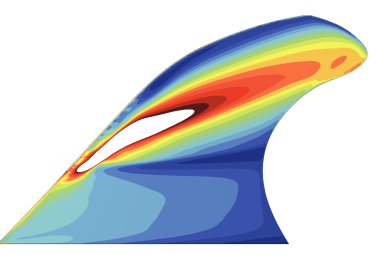

(c) $\alpha_{F}=15^{\circ}$

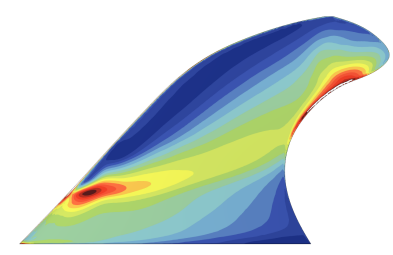

(d) $\alpha_{F}=20^{\circ}$

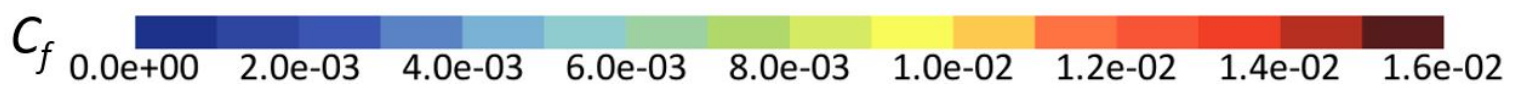

Figure B.44: $C_{f}$ coefficient contours on the suction surface, $K_{\text {taper }}=0.75$. 
B.3.3 $K_{\text {taper }}=1.00$
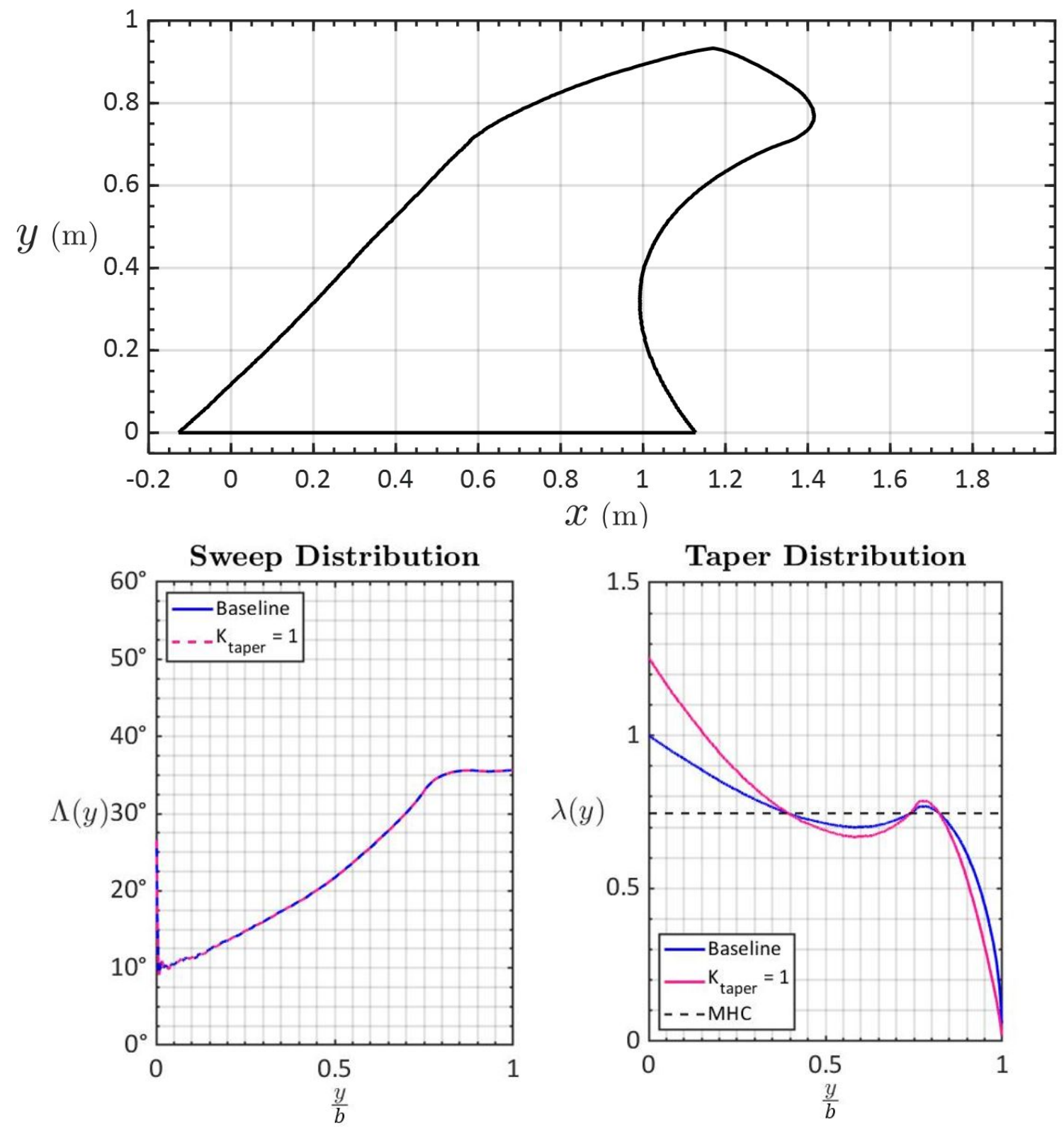

Figure B.45: Planform characteristics, $K_{\text {taper }}=1.00$. 

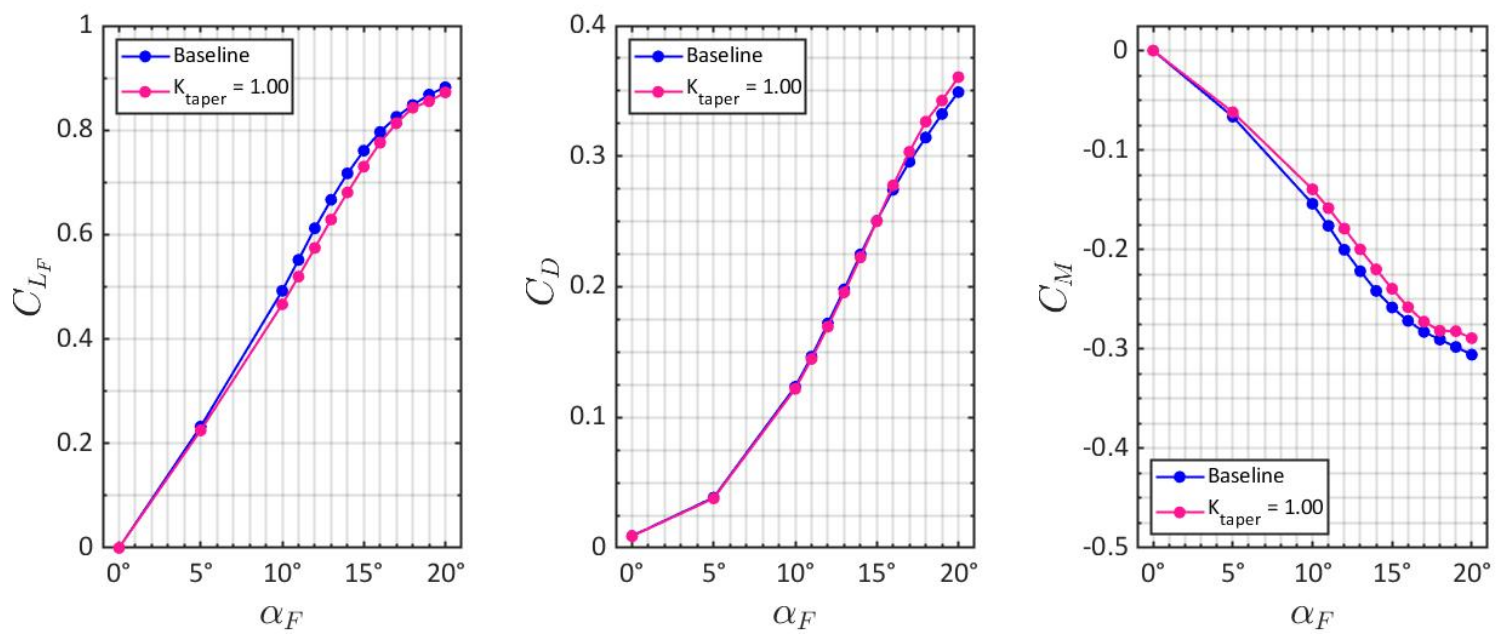

Figure B.46: Lift, drag, and moment coefficients, $K_{\text {taper }}=1.00$.

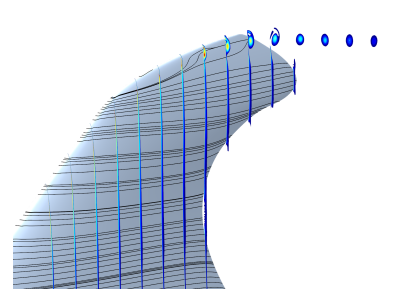

(a) $\alpha_{F}=5^{\circ}$

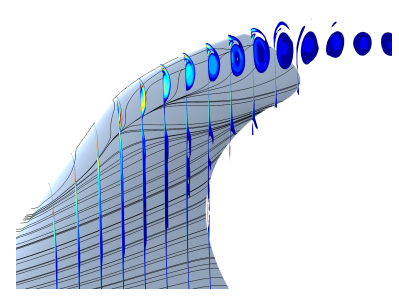

(b) $\alpha_{F}=10^{\circ}$

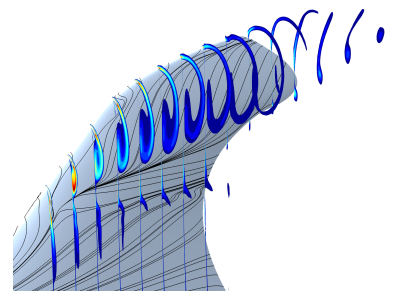

(c) $\alpha_{F}=15^{\circ}$

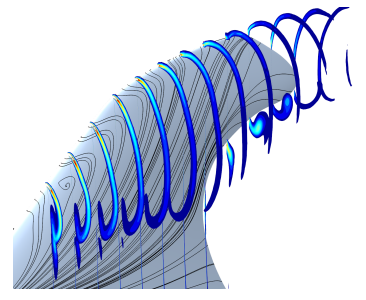

(d) $\alpha_{F}=20^{\circ}$

$|\vec{\omega}|_{10.0} \begin{array}{rrrrrrrrrr}17.0 & 24.0 & 31.0 & 38.0 & 45.0 & 52.0 & 59.0 & 66.0 & 73.0 & 80.0\end{array}$

Figure B.47: Vorticity cross sections on the suction surface, $K_{\text {taper }}=1.00$.

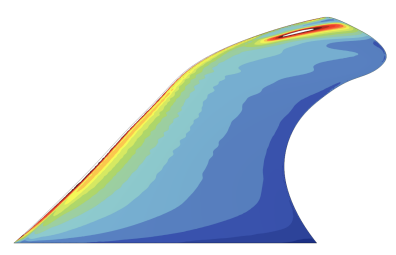

(a) $\alpha_{F}=5^{\circ}$

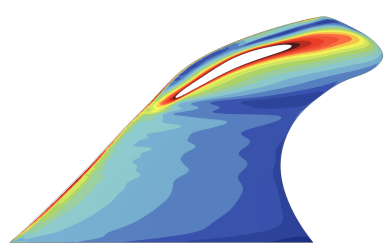

(b) $\alpha_{F}=10^{\circ}$

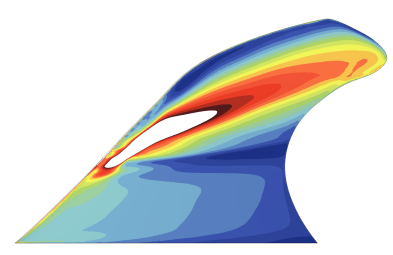

(c) $\alpha_{F}=15^{\circ}$

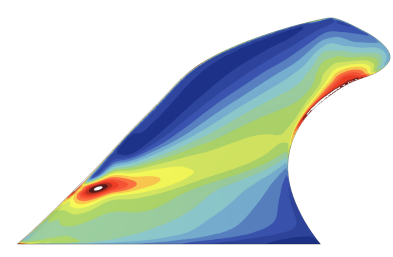

(d) $\alpha_{F}=20^{\circ}$

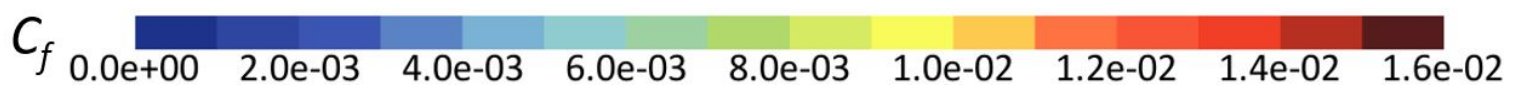

Figure B.48: $C_{f}$ coefficient contours on the suction surface, $K_{\text {taper }}=1.00$. 
B.3.4 $K_{\text {taper }}=-0.50$
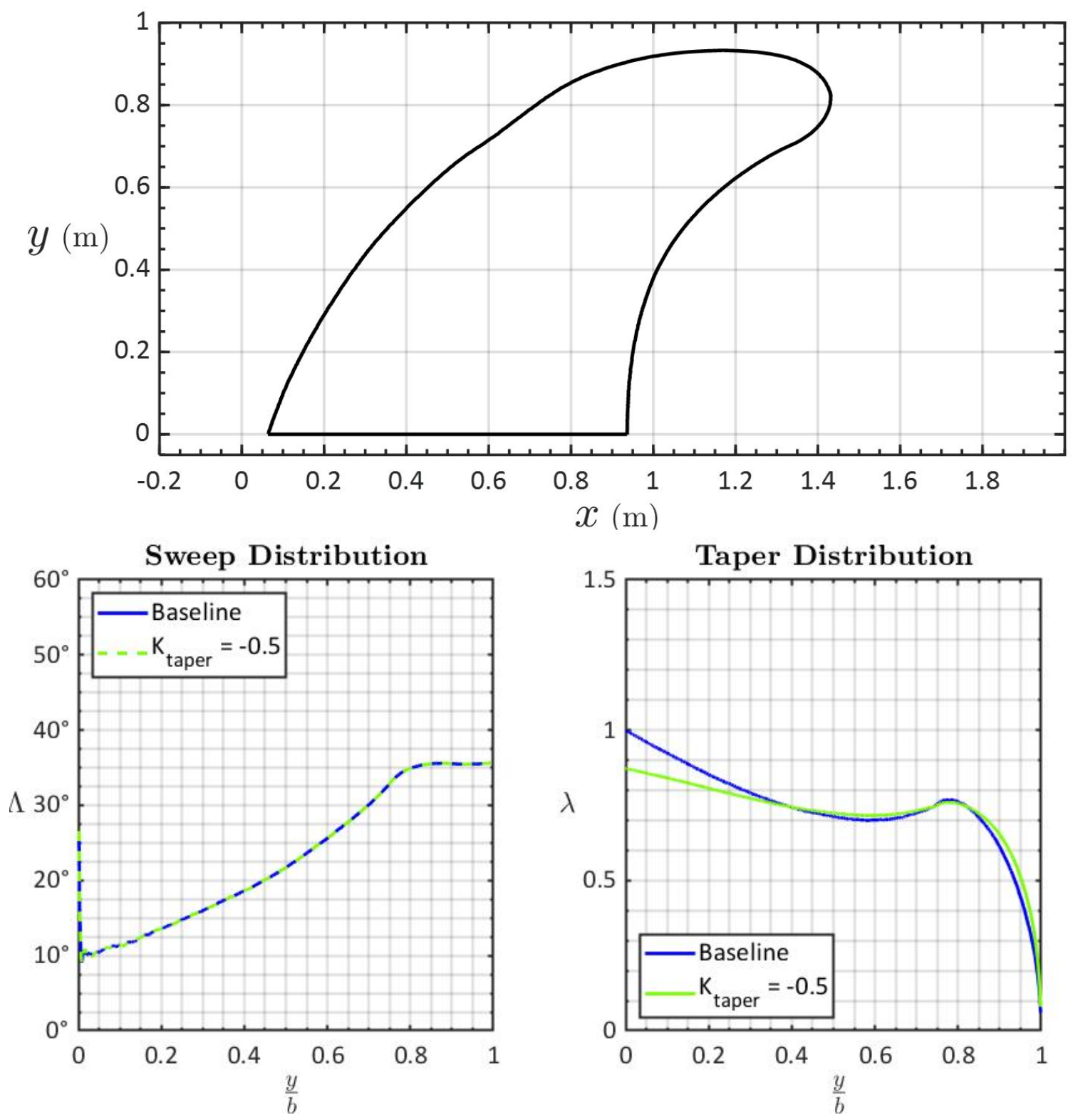

Figure B.49: Planform characteristics, $K_{\text {taper }}=-0.50$. 

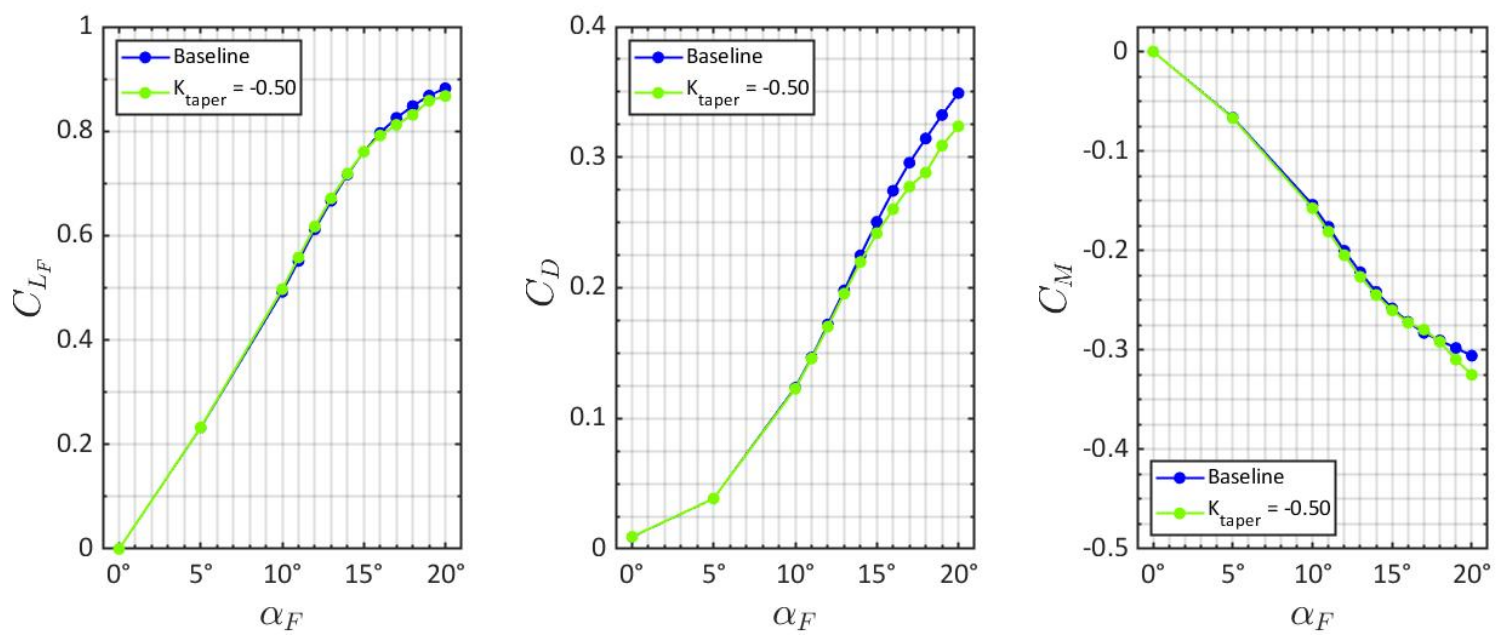

Figure B.50: Lift, drag, and moment coefficients, $K_{\text {taper }}=-0.50$.

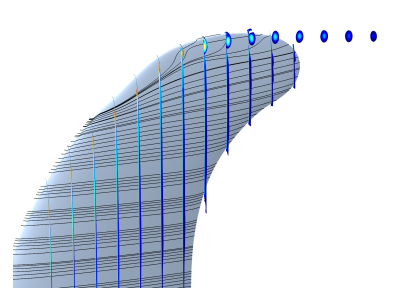

(a) $\alpha_{F}=5^{\circ}$

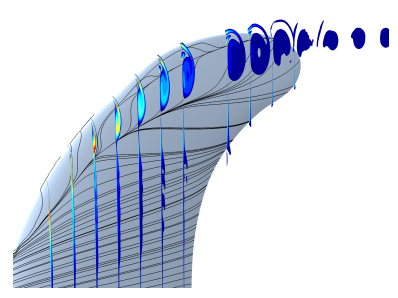

(b) $\alpha_{F}=10^{\circ}$

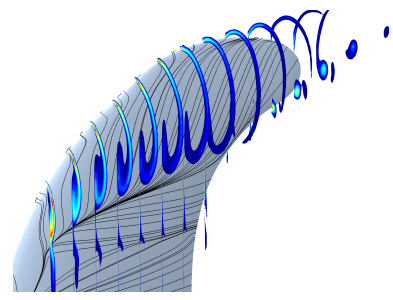

(c) $\alpha_{F}=15^{\circ}$

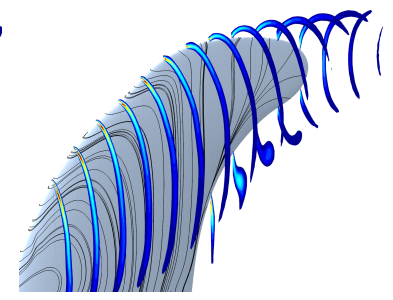

(d) $\alpha_{F}=20^{\circ}$

$|\vec{\omega}|_{10.0} \begin{array}{rrrrrrrrrr}17.0 & 24.0 & 31.0 & 38.0 & 45.0 & 52.0 & 59.0 & 66.0 & 73.0 & 80.0\end{array}$

Figure B.51: Vorticity cross sections on the suction surface, $K_{\text {taper }}=-0.50$.

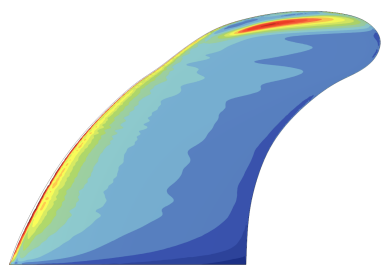

(a) $\alpha_{F}=5^{\circ}$

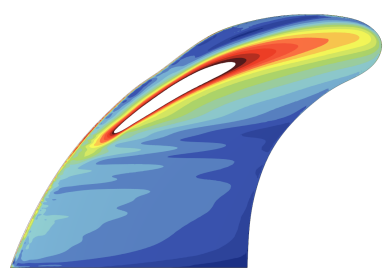

(b) $\alpha_{F}=10^{\circ}$

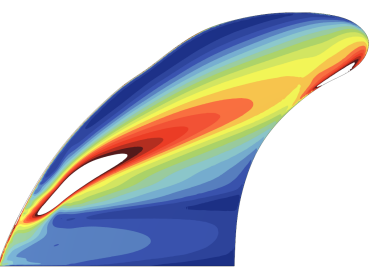

(c) $\alpha_{F}=15^{\circ}$

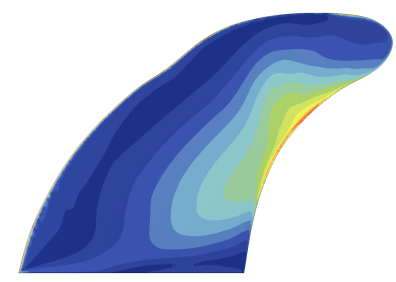

(d) $\alpha_{F}=20^{\circ}$

$\begin{array}{lllllllll}C_{f}{ }_{0.0 \mathrm{e}+00} & 2.0 \mathrm{e}-03 & 4.0 \mathrm{e}-03 & 6.0 \mathrm{e}-03 & 8.0 \mathrm{e}-03 & 1.0 \mathrm{e}-02 & 1.2 \mathrm{e}-02 & 1.4 \mathrm{e}-02 & 1.6 \mathrm{e}-02\end{array}$

Figure B.52: $C_{f}$ coefficient contours on the suction surface, $K_{\text {taper }}=-0.50$. 
B.3.5 $K_{\text {taper }}=-0.75$
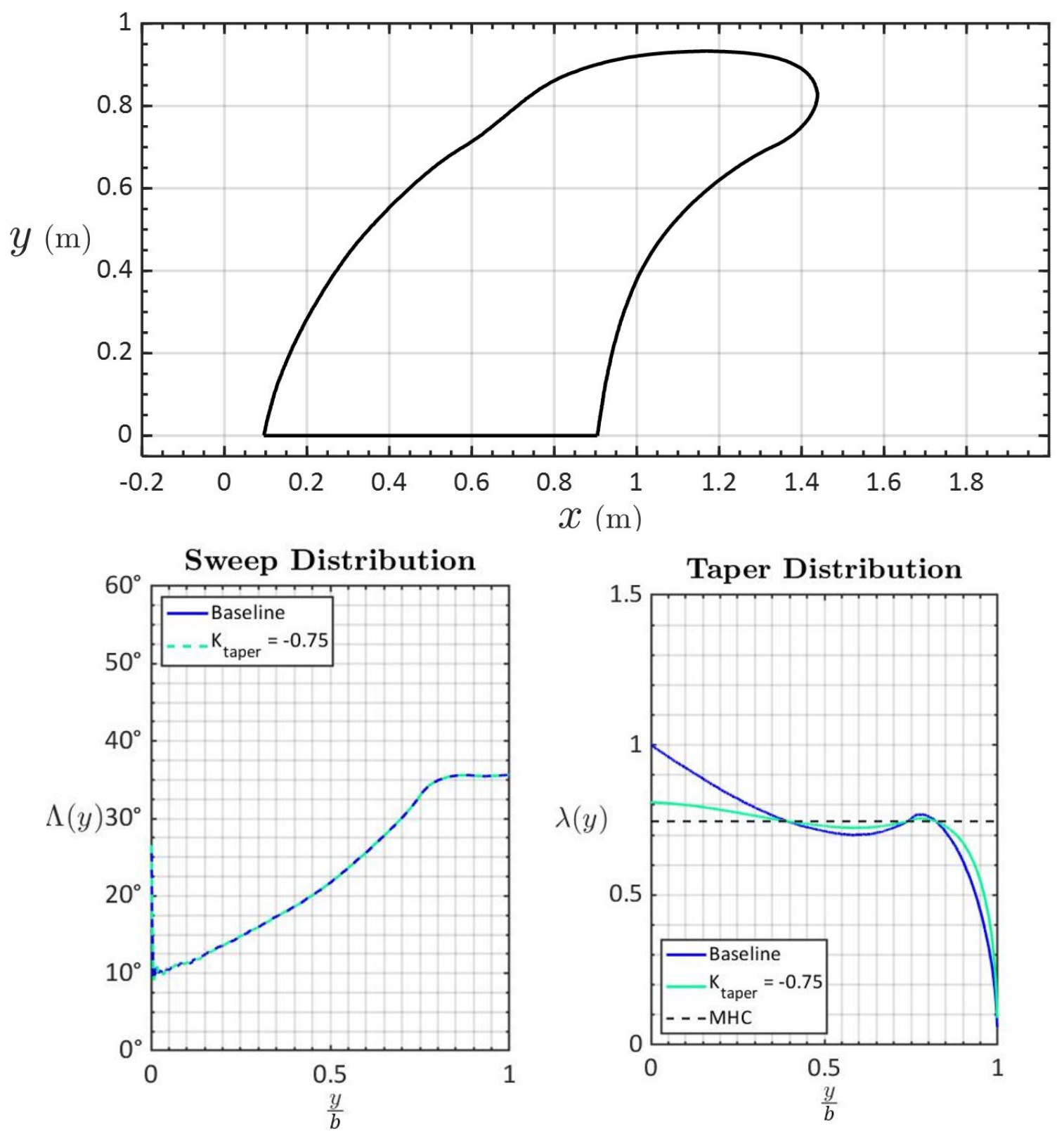

Figure B.53: Planform characteristics, $K_{\text {taper }}=-0.75$. 

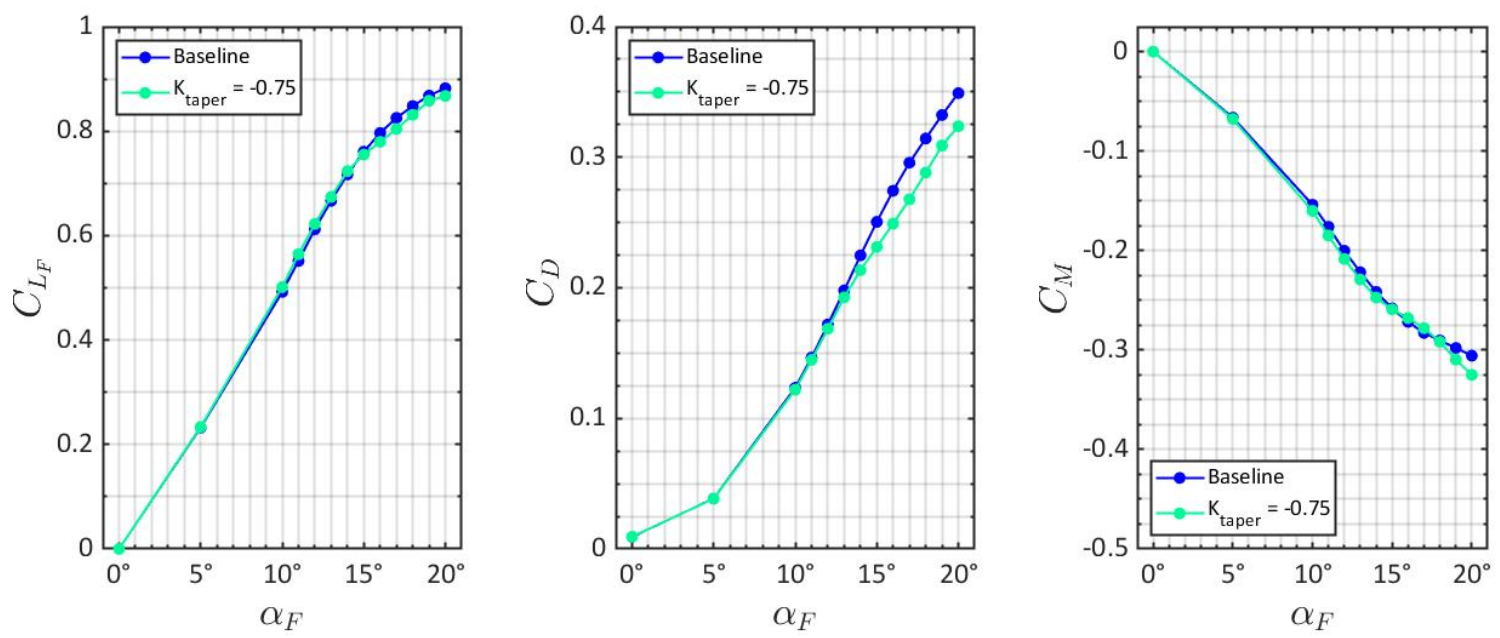

Figure B.54: Lift, drag, and moment coefficients, $K_{\text {taper }}=-0.75$.

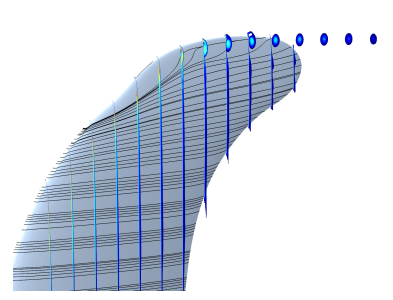

(a) $\alpha_{F}=5^{\circ}$

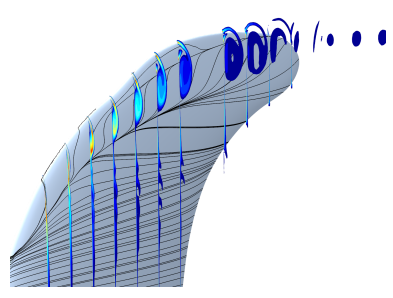

(b) $\alpha_{F}=10^{\circ}$

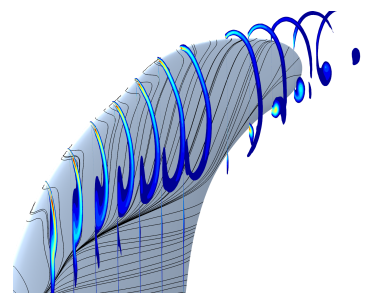

(c) $\alpha_{F}=15^{\circ}$

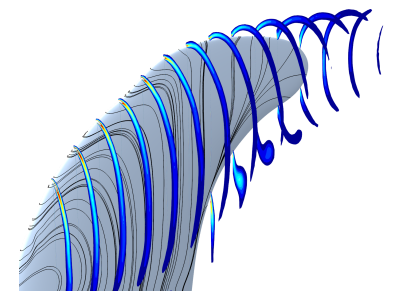

(d) $\alpha_{F}=20^{\circ}$

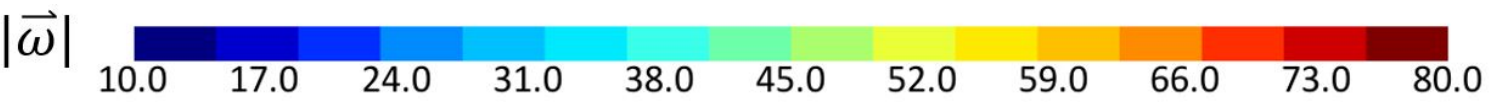

Figure B.55: Vorticity cross sections on the suction surface, $K_{\text {taper }}=-0.75$.

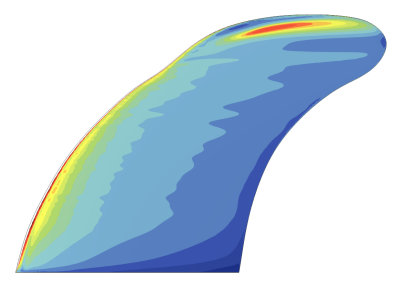

(a) $\alpha_{F}=5^{\circ}$

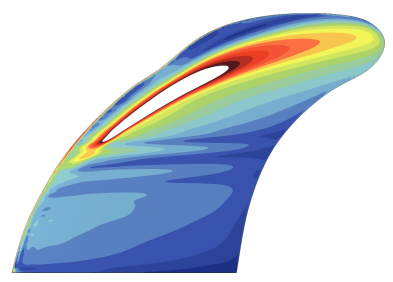

(b) $\alpha_{F}=10^{\circ}$

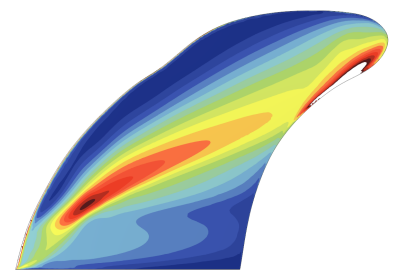

(c) $\alpha_{F}=15^{\circ}$

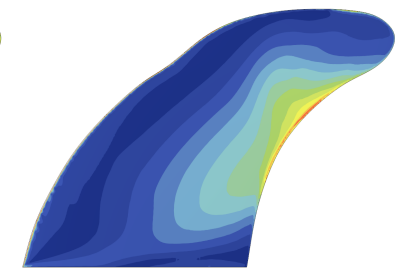

(d) $\alpha_{F}=20^{\circ}$

$\begin{array}{lllllllll}C_{f}{ }_{0.0 \mathrm{e}+00} & 2.0 \mathrm{e}-03 & 4.0 \mathrm{e}-03 & 6.0 \mathrm{e}-03 & 8.0 \mathrm{e}-03 & 1.0 \mathrm{e}-02 & 1.2 \mathrm{e}-02 & 1.4 \mathrm{e}-02 & 1.6 \mathrm{e}-02\end{array}$

Figure B.56: $C_{f}$ coefficient contours on the suction surface, $K_{\text {taper }}=-0.75$. 
B.3.6 $K_{\text {taper }}=-1.00$
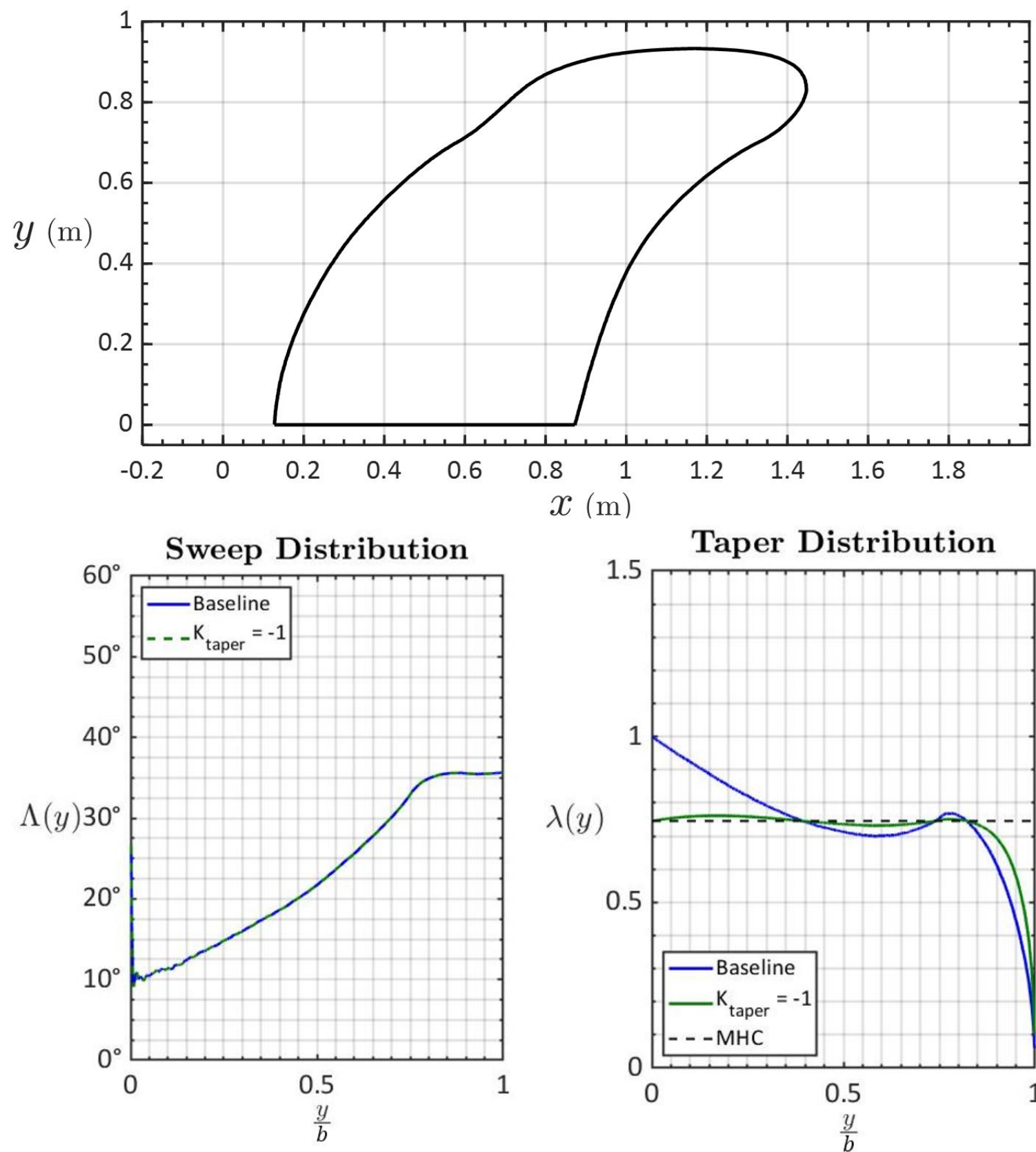

Taper Distribution

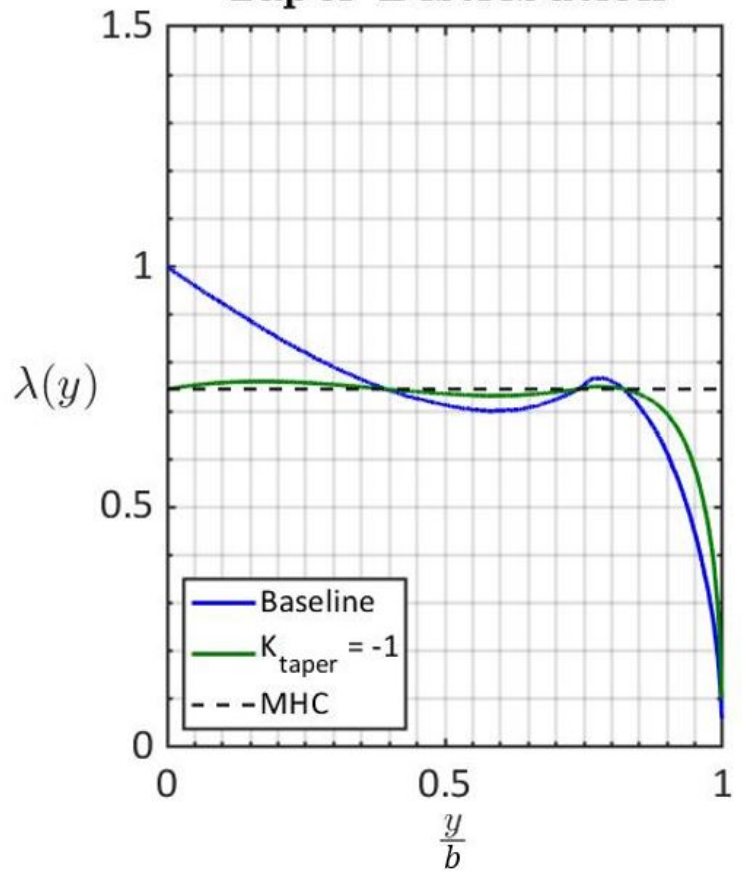

Figure B.57: Planform characteristics, $K_{\text {taper }}=1.00$. 

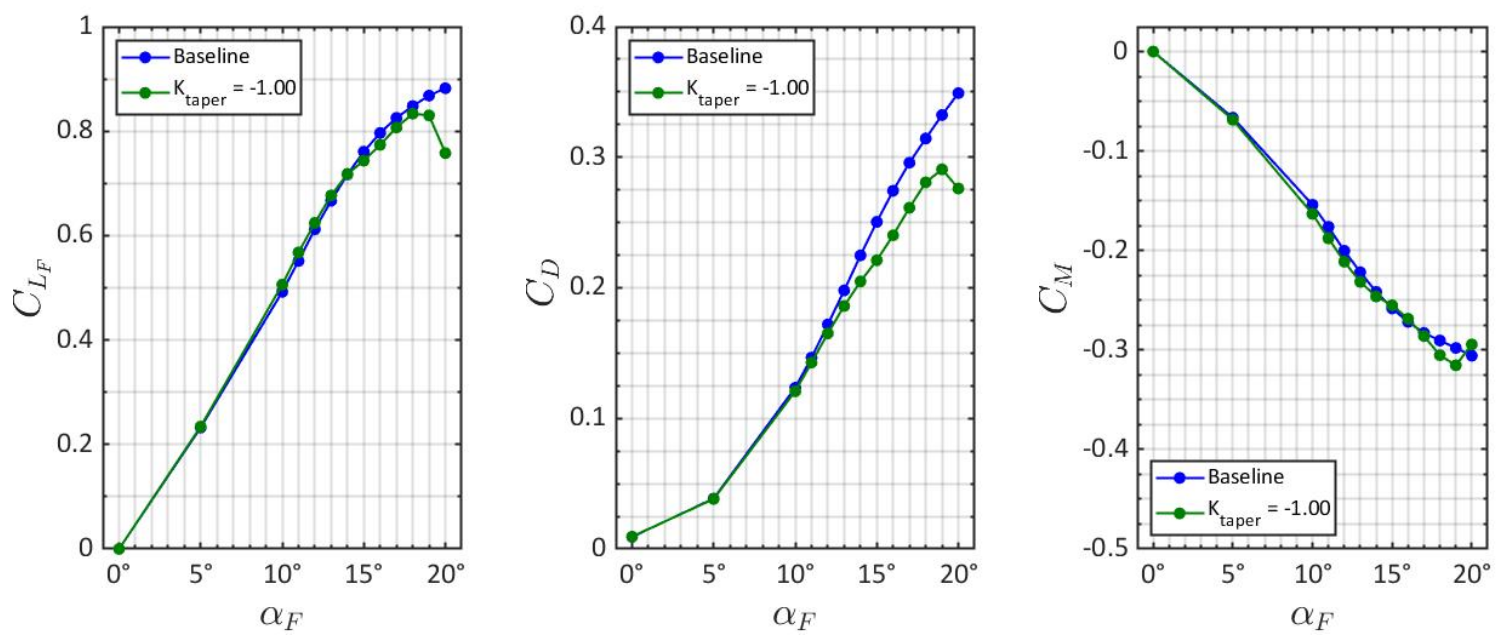

Figure B.58: Lift, drag, and moment coefficients, $K_{\text {taper }}=-1.00$.

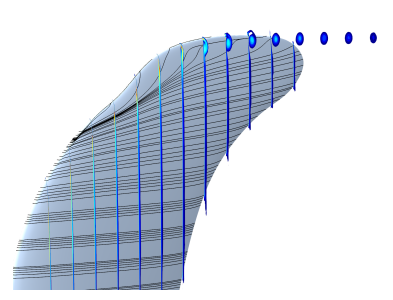

(a) $\alpha_{F}=5^{\circ}$

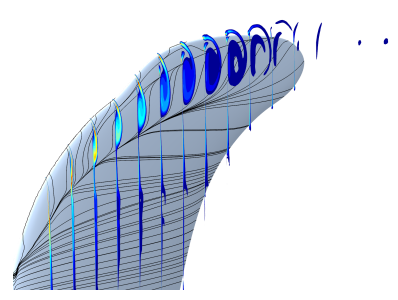

(b) $\alpha_{F}=10^{\circ}$

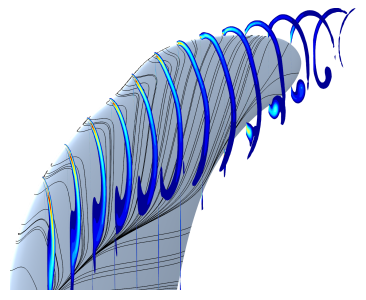

(c) $\alpha_{F}=15^{\circ}$

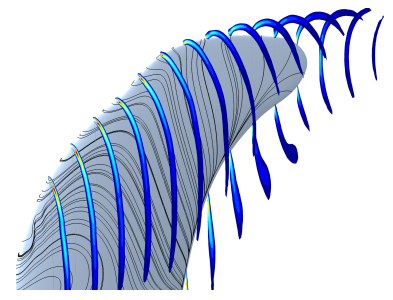

(d) $\alpha_{F}=20^{\circ}$

$|\vec{\omega}|$

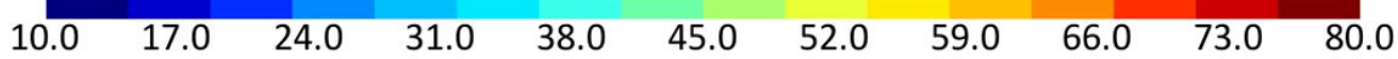

Figure B.59: Vorticity cross sections on the suction surface, $K_{\text {taper }}=-1.00$.

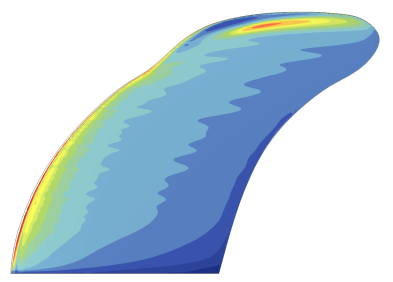

(a) $\alpha_{F}=5^{\circ}$

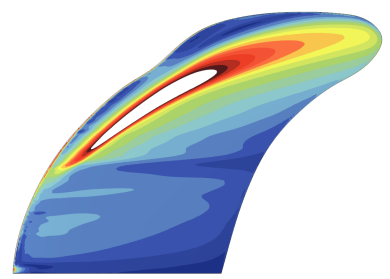

(b) $\alpha_{F}=10^{\circ}$

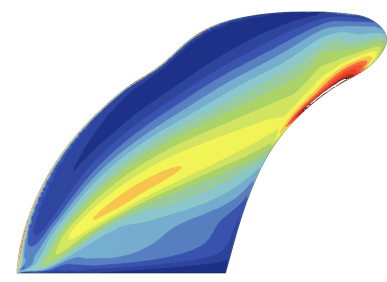

(c) $\alpha_{F}=15^{\circ}$

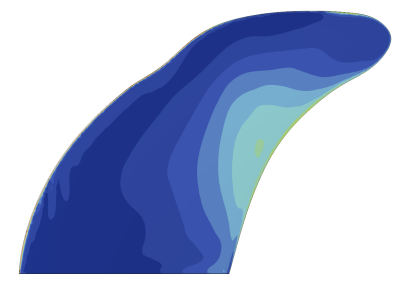

(d) $\alpha_{F}=20^{\circ}$

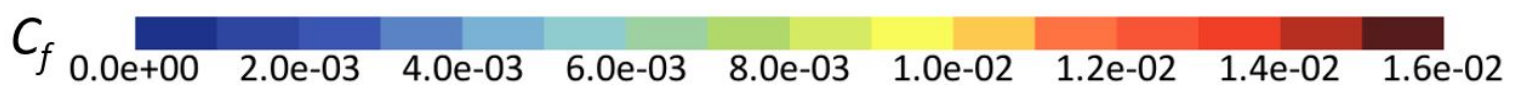

Figure B.60: $C_{f}$ coefficient contours on the suction surface, $K_{\text {taper }}=-1.00$. 


\section{B.4 Proportionally Tapered}

The following sets of fin data were not included in the body of the report because the surface area is not constant. Each fin shape was created using Equation 4.14.

B.4.1 $K_{\text {taper }}=0.50$
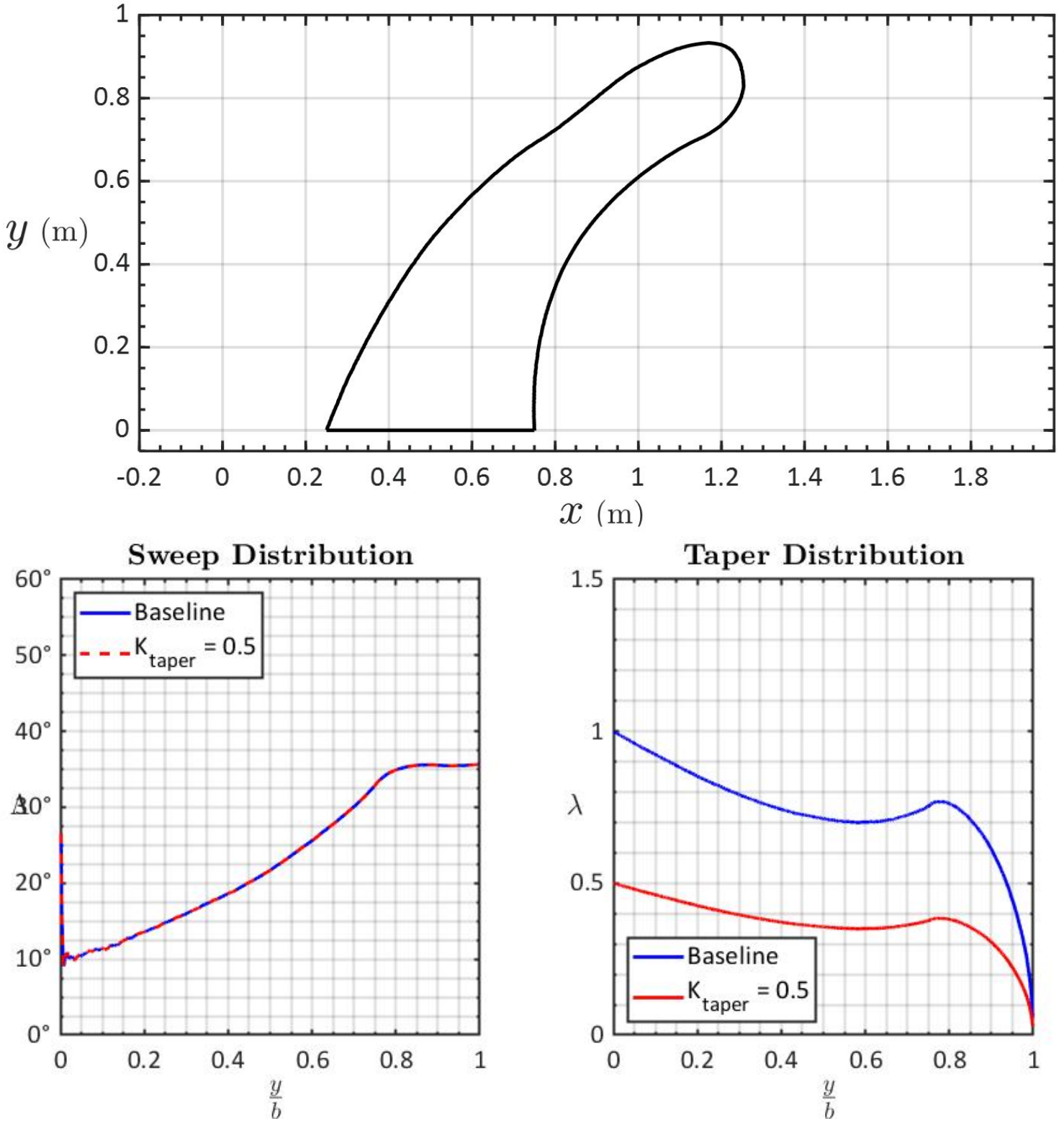

Figure B.61: Planform characteristics, $K_{\text {taper }}=0.50$. 

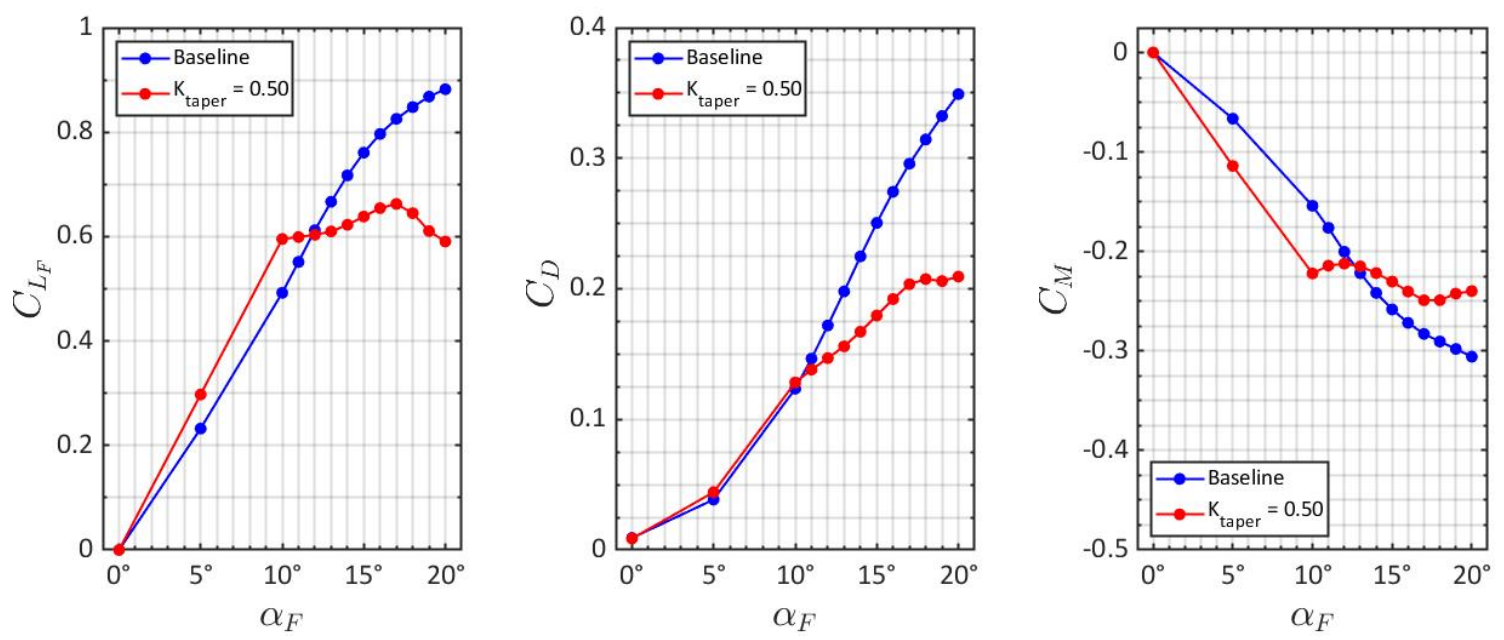

Figure B.62: Lift, drag, and moment coefficients, $K_{\text {taper }}=0.50$.

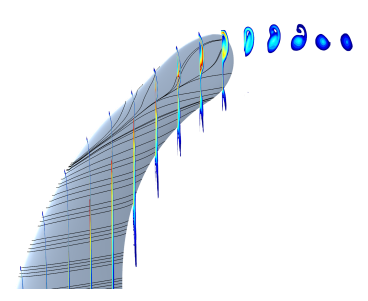

(a) $\alpha_{F}=5^{\circ}$

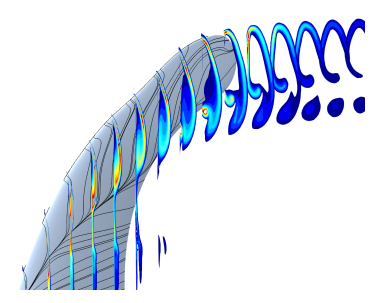

(b) $\alpha_{F}=10^{\circ}$

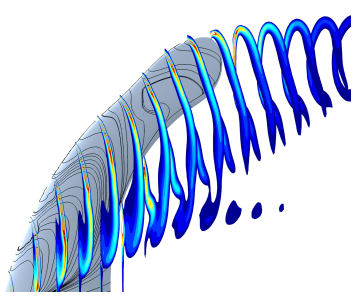

(c) $\alpha_{F}=15^{\circ}$

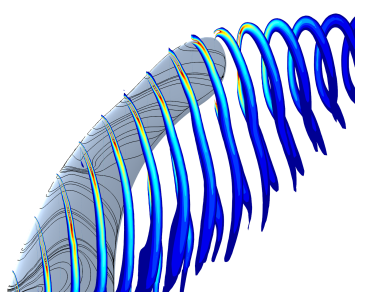

(d) $\alpha_{F}=20^{\circ}$

$|\vec{\omega}|$

$\begin{array}{lllllllllll}10.0 & 17.0 & 24.0 & 31.0 & 38.0 & 45.0 & 52.0 & 59.0 & 66.0 & 73.0 & 80.0\end{array}$

Figure B.63: Vorticity cross sections on the suction surface, $K_{\text {taper }}=0.50$.

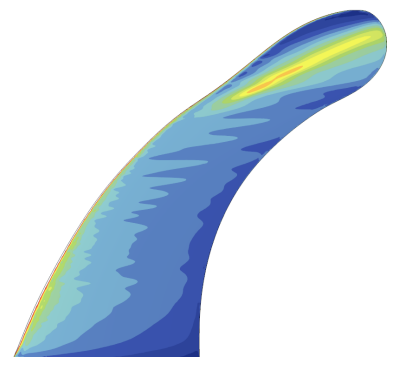

(a) $\alpha_{F}=5^{\circ}$

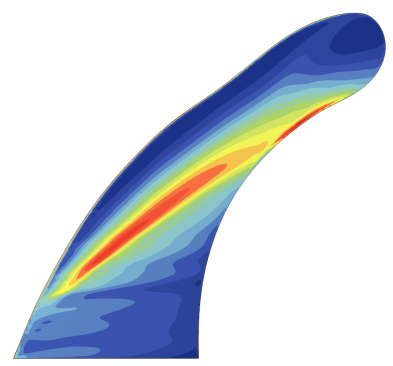

(b) $\alpha_{F}=10^{\circ}$

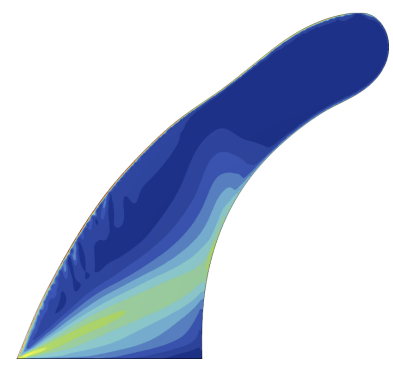

(c) $\alpha_{F}=15^{\circ}$

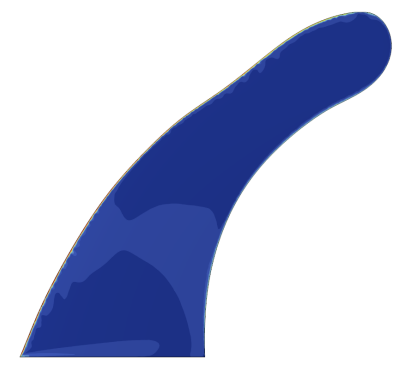

(d) $\alpha_{F}=20^{\circ}$

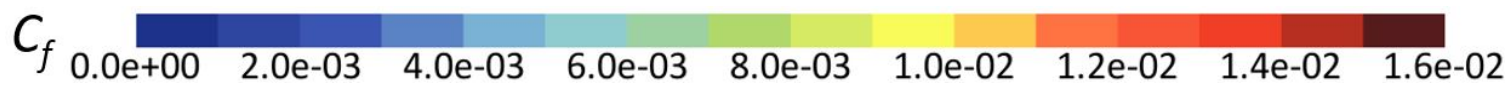

Figure B.64: $C_{f}$ coefficient contours on the suction surface, $K_{\text {taper }}=0.50$. 
B.4.2 $K_{\text {taper }}=0.75$
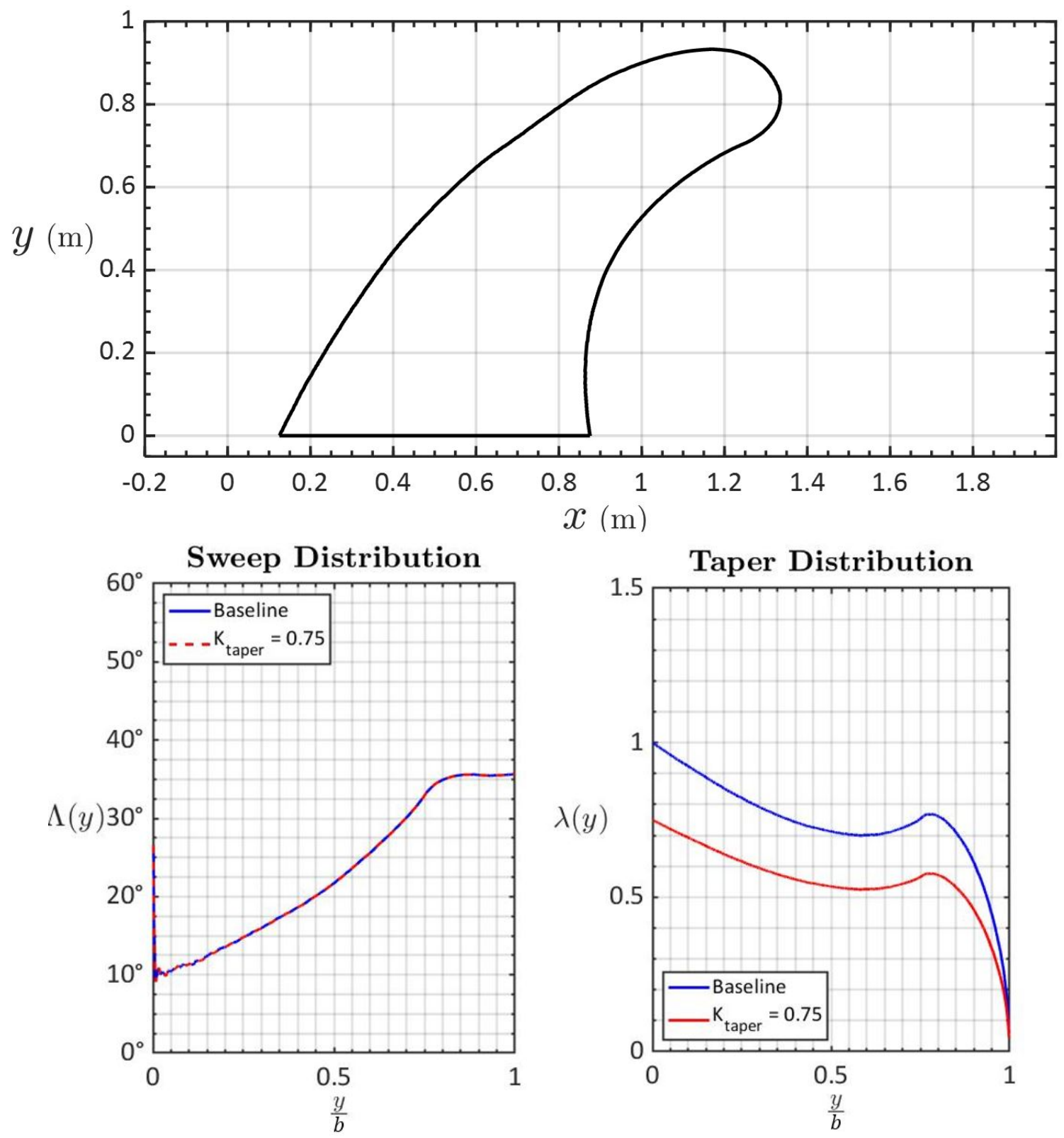

Figure B.65: Planform characteristics, $K_{\text {taper }}=0.75$. 

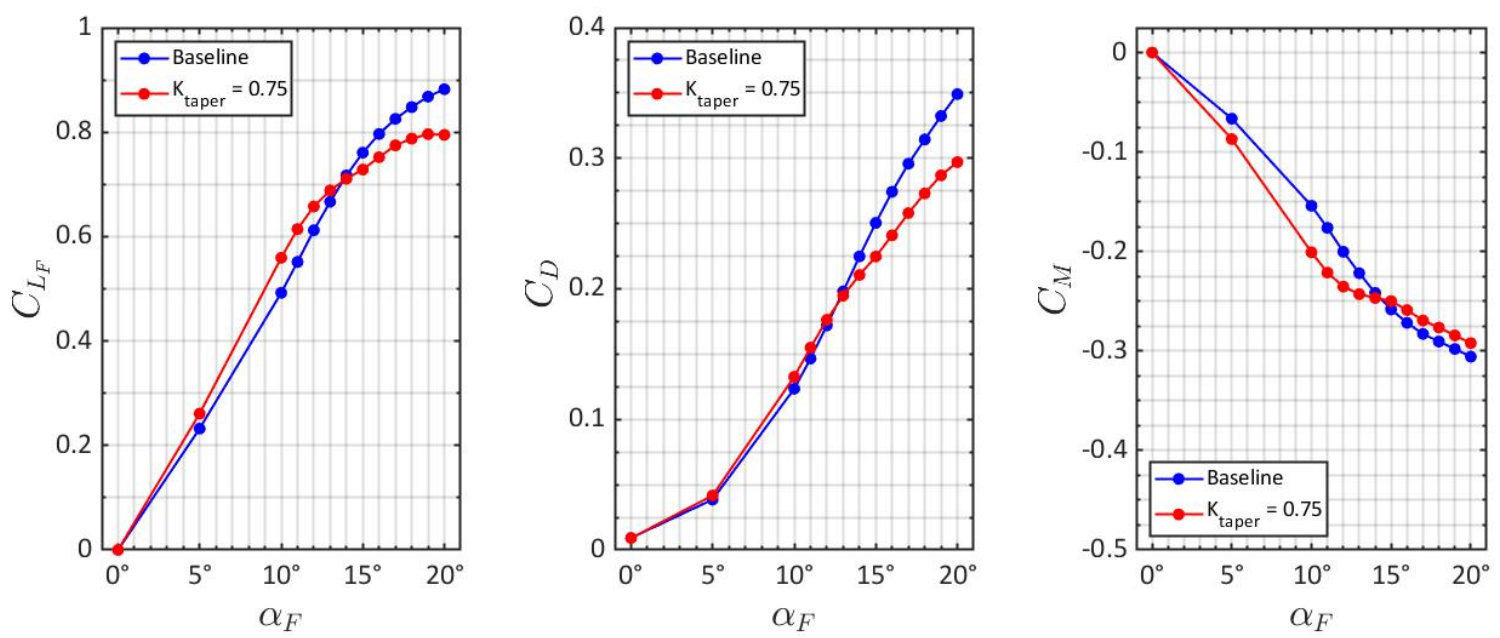

Figure B.66: Lift, drag, and moment coefficients, $K_{\text {taper }}=0.75$.

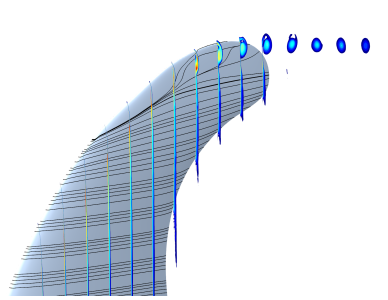

(a) $\alpha_{F}=5^{\circ}$

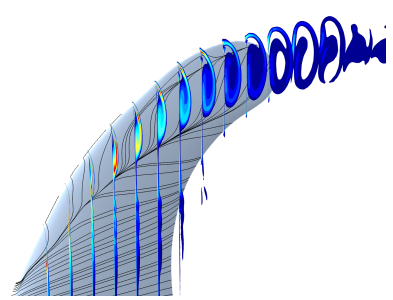

(b) $\alpha_{F}=10^{\circ}$

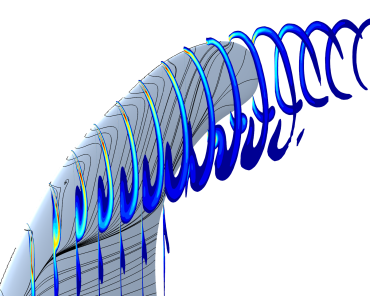

(c) $\alpha_{F}=15^{\circ}$

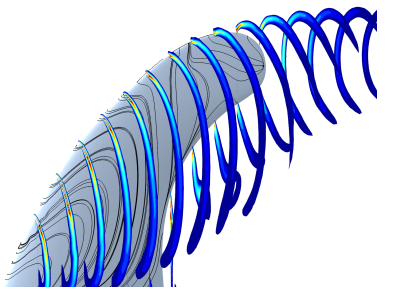

(d) $\alpha_{F}=20^{\circ}$

\section{$|\vec{\omega}|_{10.0} \begin{array}{rrrrrrrrrr}17.0 & 24.0 & 31.0 & 38.0 & 45.0 & 52.0 & 59.0 & 66.0 & 73.0 & 80.0\end{array}$}

Figure B.67: Vorticity cross sections on the suction surface, $K_{\text {taper }}=0.75$.

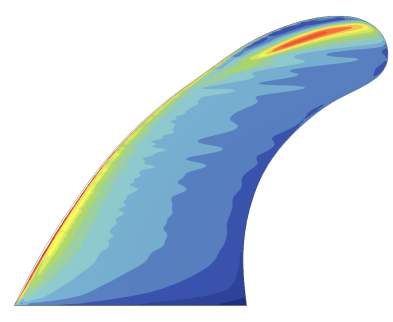

(a) $\alpha_{F}=5^{\circ}$

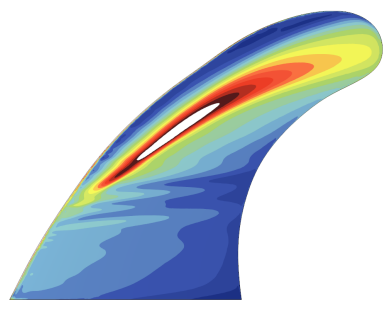

(b) $\alpha_{F}=10^{\circ}$

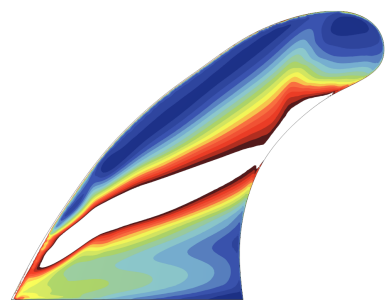

(c) $\alpha_{F}=15^{\circ}$

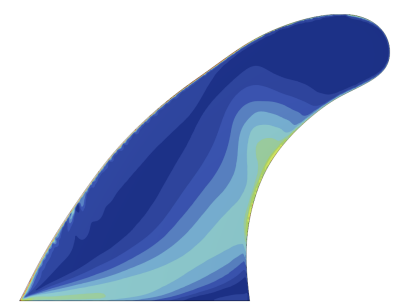

(d) $\alpha_{F}=20^{\circ}$

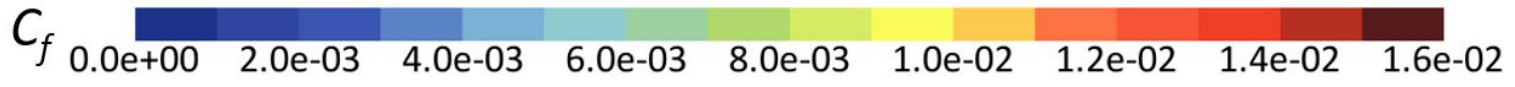

Figure B.68: $C_{f}$ coefficient contours on the suction surface, $K_{\text {taper }}=0.75$. 
B.4.3 $K_{\text {taper }}=1.25$
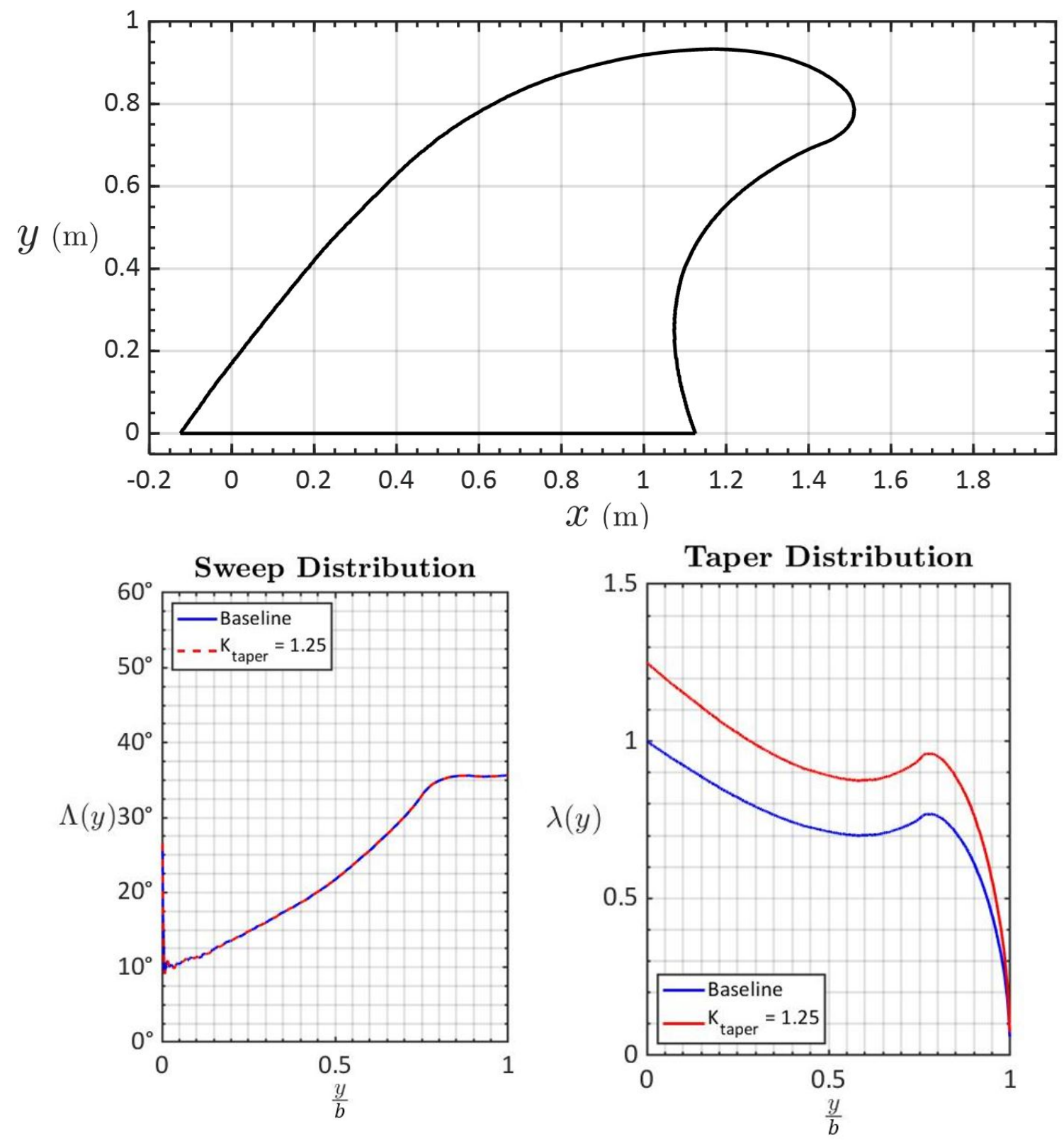

Figure B.69: Planform characteristics, $K_{\text {taper }}=1.25$. 

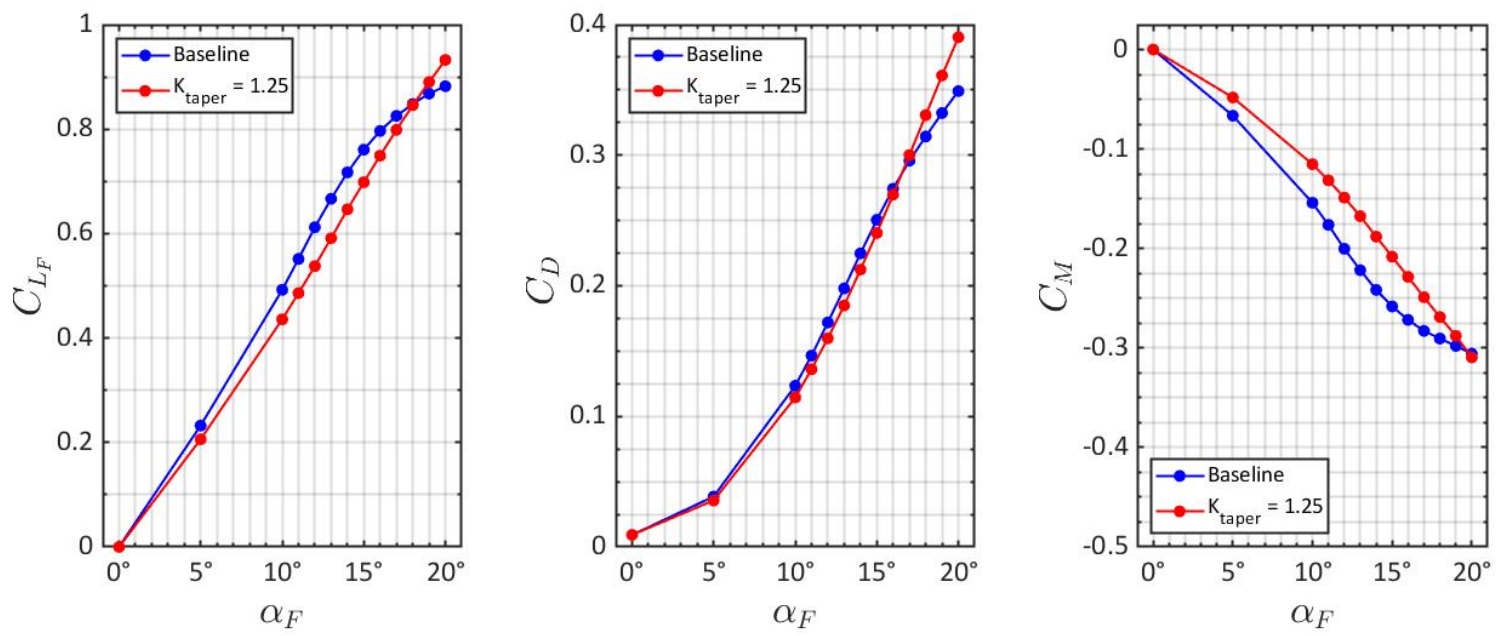

Figure B.70: Lift, drag, and moment coefficients, $K_{\text {taper }}=1.25$.

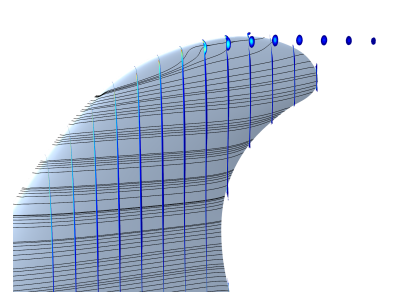

(a) $\alpha_{F}=5^{\circ}$

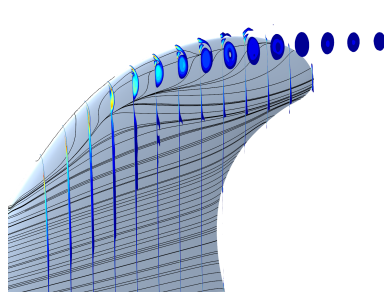

(b) $\alpha_{F}=10^{\circ}$

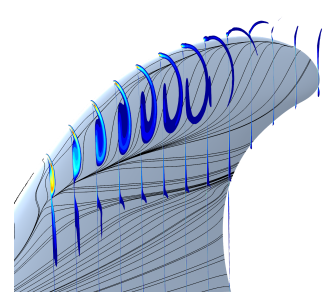

(c) $\alpha_{F}=15^{\circ}$

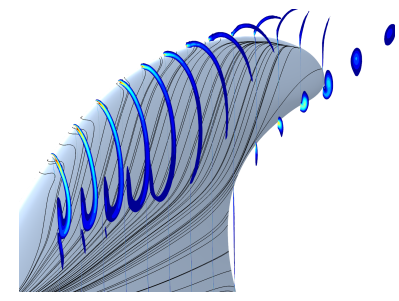

(d) $\alpha_{F}=20^{\circ}$

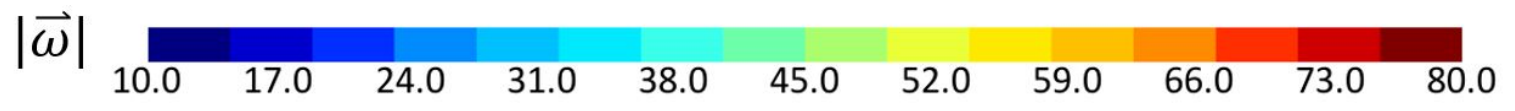

Figure B.71: Vorticity cross sections on the suction surface, $K_{\text {taper }}=1.25$.

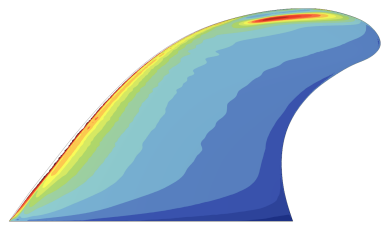

(a) $\alpha_{F}=5^{\circ}$

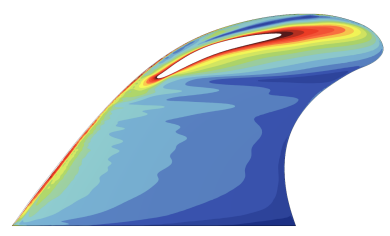

(b) $\alpha_{F}=10^{\circ}$

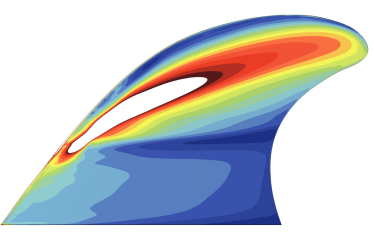

(c) $\alpha_{F}=15^{\circ}$

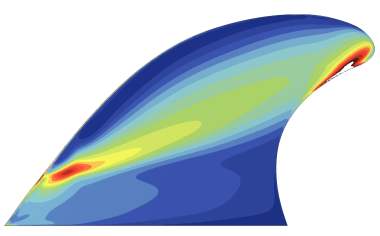

(d) $\alpha_{F}=20^{\circ}$

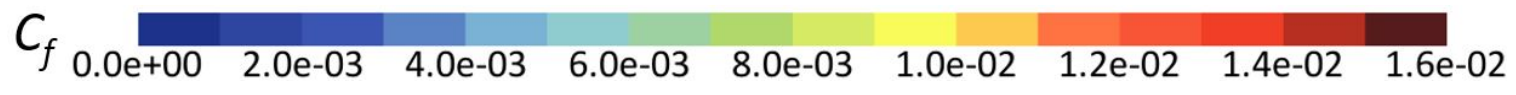

Figure B.72: $C_{f}$ coefficient contours on the suction surface, $K_{\text {taper }}=1.25$. 
B.4.4 $K_{\text {taper }}=1.50$
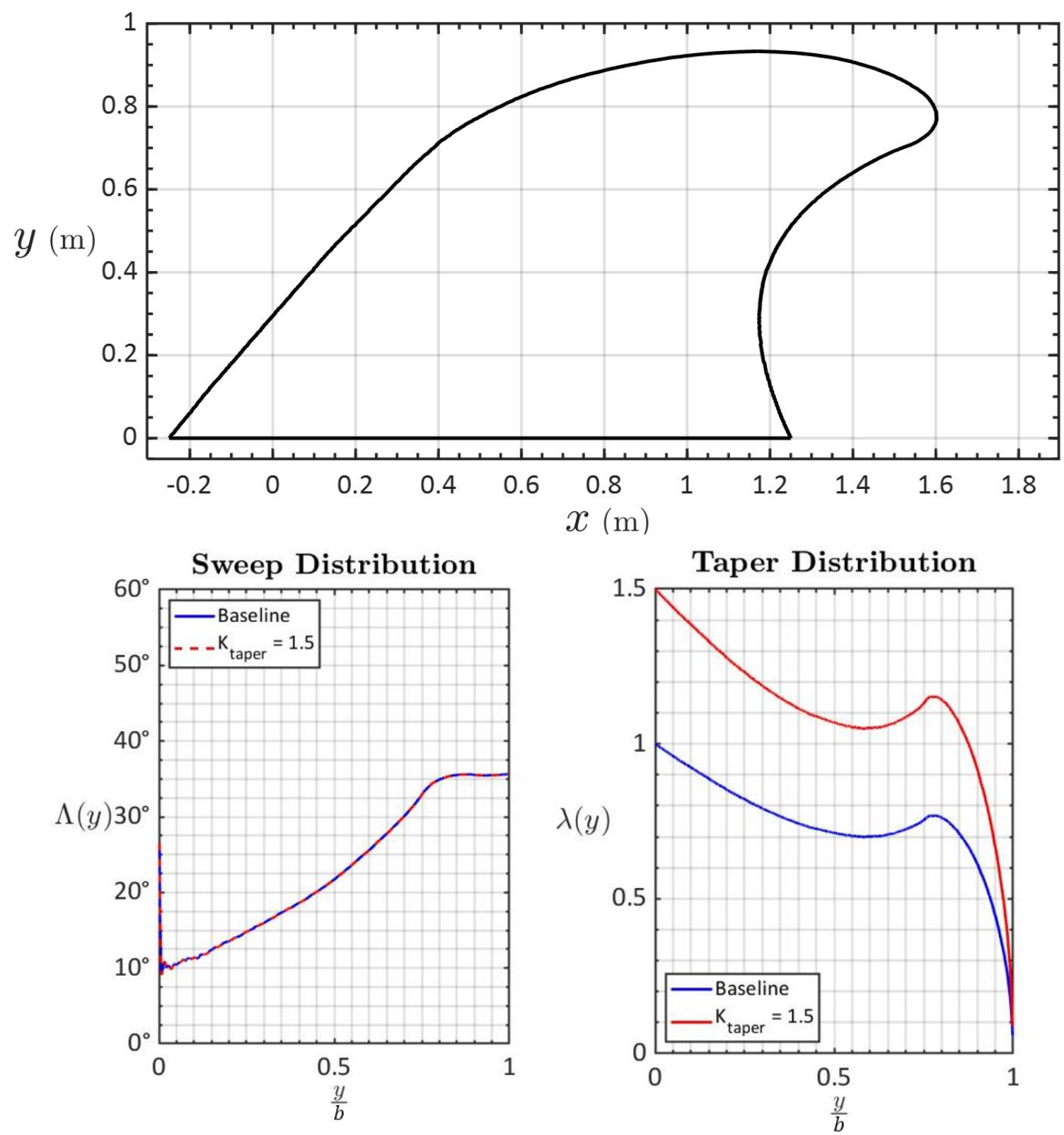

Figure B.73: Planform characteristics, $K_{\text {taper }}=1.50$. 

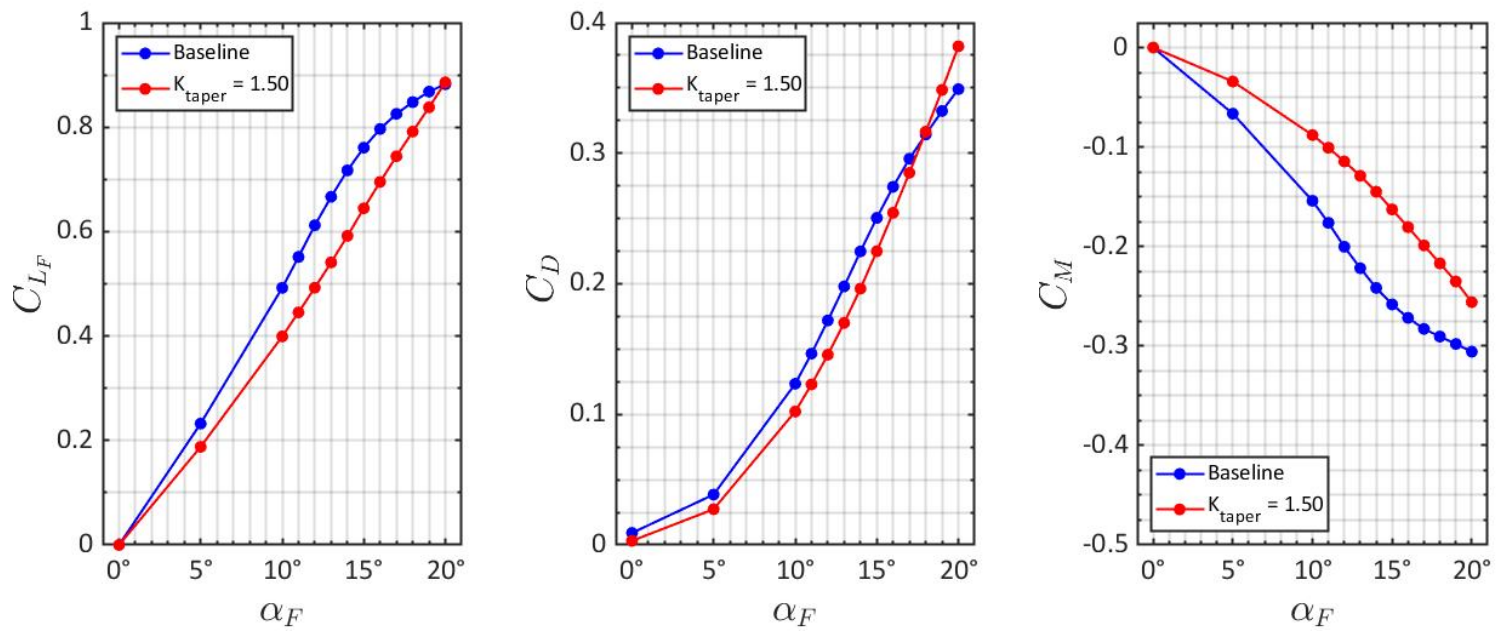

Figure B.74: Lift, drag, and moment coefficients, $K_{\text {taper }}=1.50$.

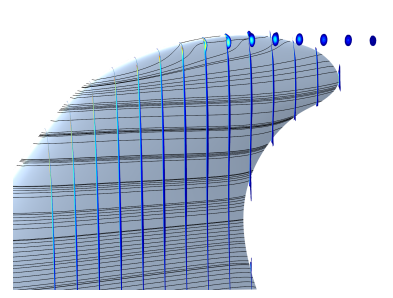

(a) $\alpha_{F}=5^{\circ}$

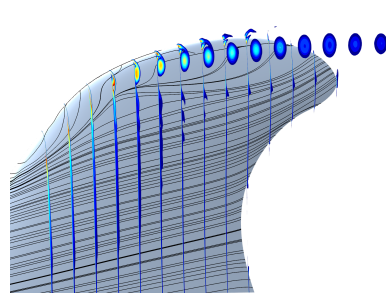

(b) $\alpha_{F}=10^{\circ}$

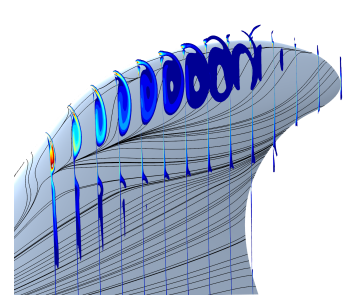

(c) $\alpha_{F}=15^{\circ}$

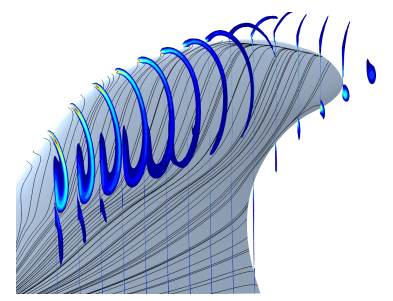

(d) $\alpha_{F}=20^{\circ}$

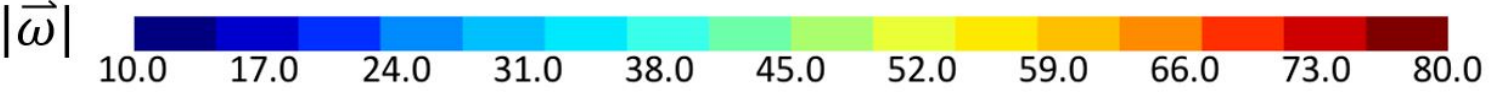

Figure B.75: Vorticity cross sections on the suction surface, $K_{\text {taper }}=1.50$.

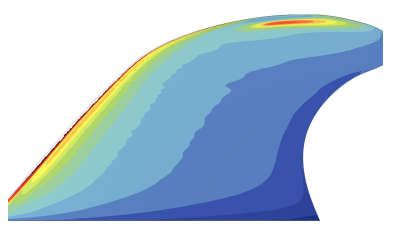

(a) $\alpha_{F}=5^{\circ}$

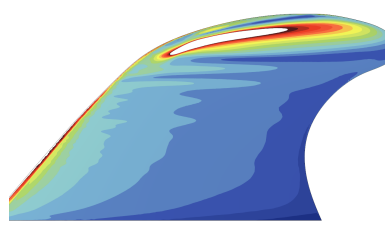

(b) $\alpha_{F}=10^{\circ}$

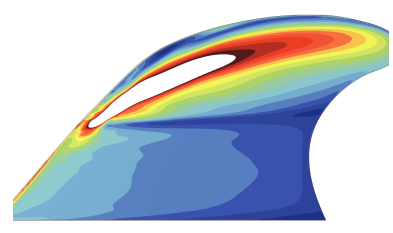

(c) $\alpha_{F}=15^{\circ}$

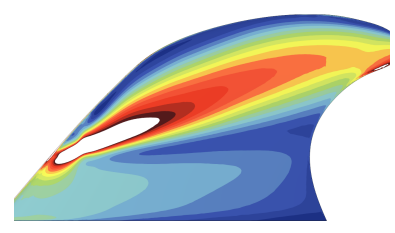

(d) $\alpha_{F}=20^{\circ}$

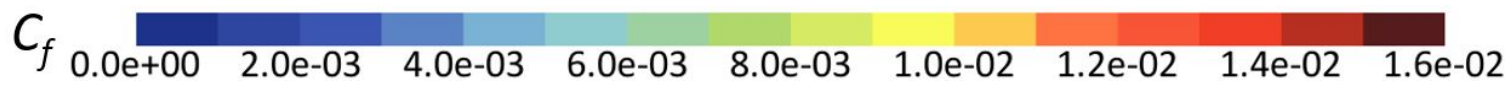

Figure B.76: $C_{f}$ coefficient contours on the suction surface, $K_{\text {taper }}=1.50$. 


\section{Appendix C}

CODE

\section{C.1 Matlab Code}

\section{C.1.1 Fin Planform Generation}

The below code was used to generate the fin planform shapes. It should be noted that on line 23, the baseline image is loaded, and the name used was the name of the file saved locally.

1 \% Brandon Baldovin

2 October 2018

$3 \quad \mathrm{clc}$;

4 clearvars;

5

$6 \%$ Description

7 \%mage processing to find the outline of a surfboard fin .

Once the outline

$8 \%$ is saved in $x, y$ coordinates, the script backs out the centerline of the

9 \%fin and tries to create an equation for it

10

${ }_{11} \%$ Flags

12 remove_flag $=$ false $\quad$ \%Remove Duplicate Points

13 sweep_flag $=$ false $; \quad$ \%Changes Sweep

14

taper_flag = false; $\quad$ \%Changes Taper

${ }_{15}$ CSV_Write = false ; $\quad \%$ Writes CSV File 
${ }_{17} \%$ Fin Parameters Change

18 SweepNew $=15 ; \quad \%$ New Max Sweep (deg)

19 TaperConstant $=1$;

$\%$ Ratio of New to Old Taper

20

$21 \%$ Border

$22 \%$ Load Image

${ }_{23} \mathrm{RGB}=$ imread ('FCS_FT_BLACK. png') ;

${ }_{24} \mathrm{I}=\operatorname{rgb} 2 \operatorname{gray}(\mathrm{RGB})$;

${ }_{25} \%$ Turns image into binary

${ }_{26} \mathrm{BW}=\mathrm{imbinarize}(\mathrm{I})$;

27

28 \%ompare the Original Image with the Binary

29 imshowpair ( I ,BW, 'montage')

30 


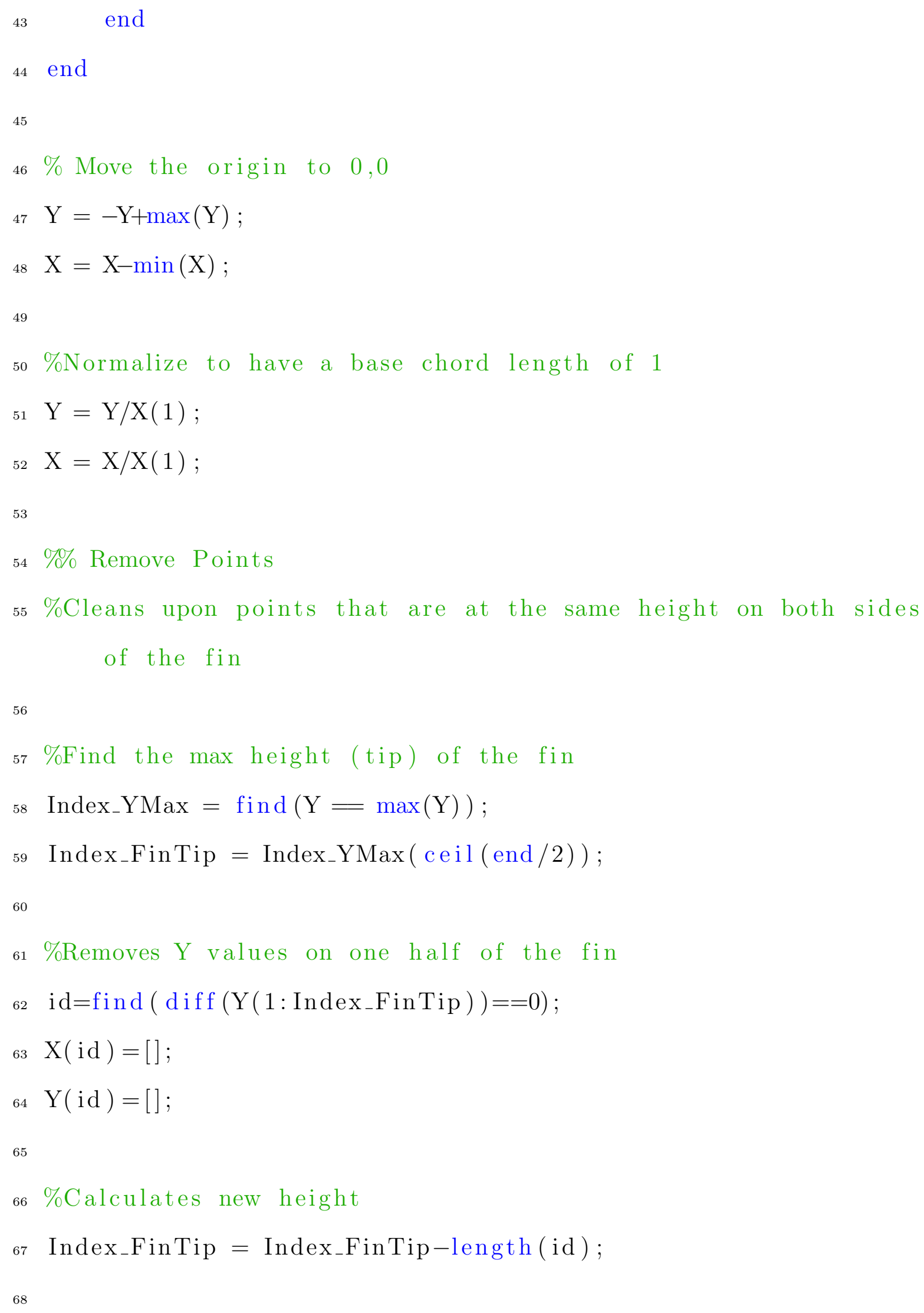




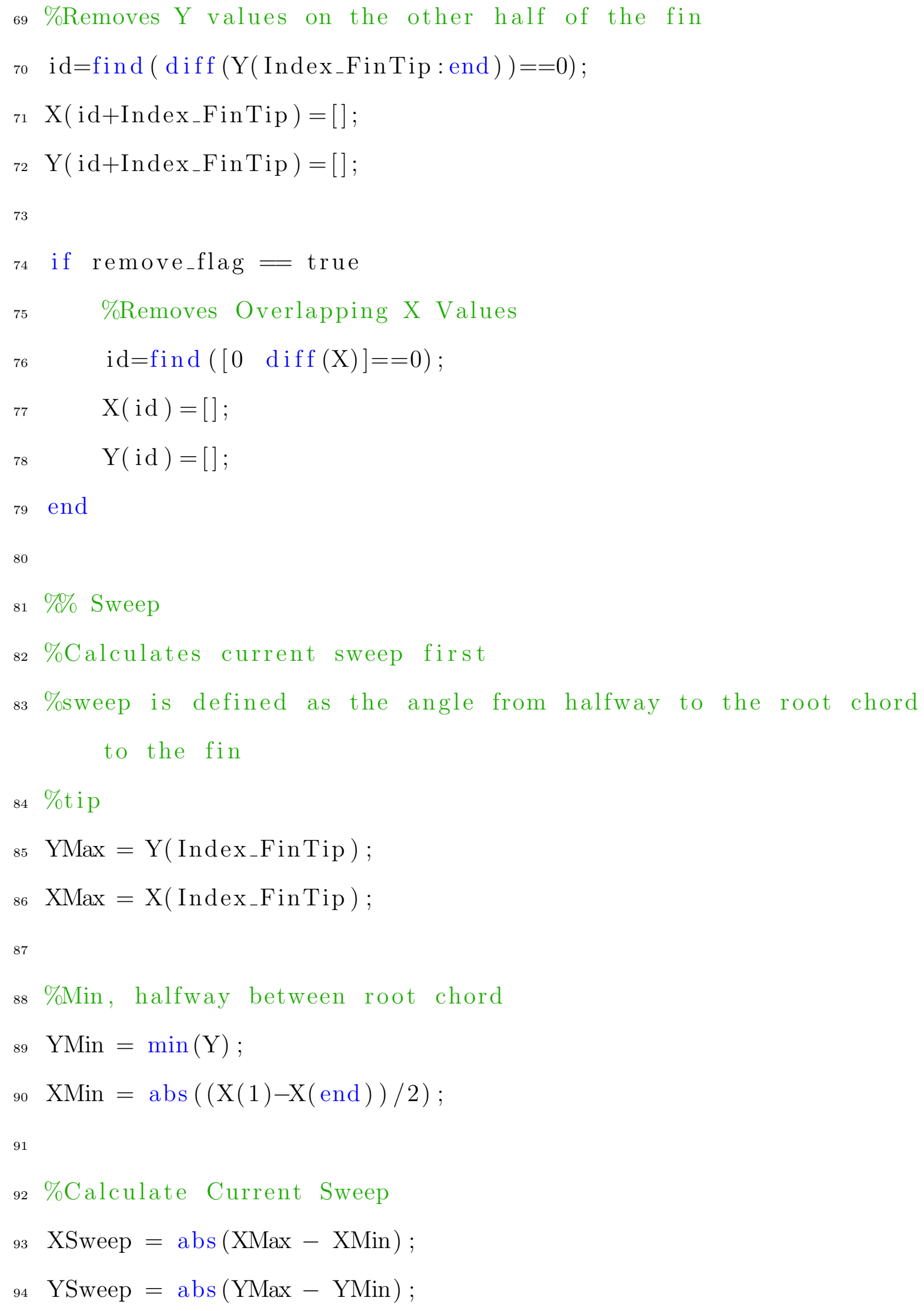


96

97

98

99

100

101

102

103

104

105

106

107

108

\% \% Taper

${ }_{110} \%$ Calculate the taper along the fin

111

112

113

114

115

116

117

118

119

120

SweepCurrent $=$ atand $($ XSweep $/$ YSweep $) ;$

SweepFin $=$ zeros $(1$, Index_FinTip -2$)$; you need to

\%start one up from root chord

for i i $=2$ : Index_FinTip -1

Ytemp $=\operatorname{abs}(Y($ i i $)-$ YMin $)$;

SweepFin $($ i -1$)=\operatorname{atand}($ Xtemp/Ytemp $)$;

end

SweepFin $=\operatorname{smooth}($ flip $($ SweepFin $)$, 'moving' $)$;

$\mathrm{XRoot}=\operatorname{abs}(\mathrm{X}(1)-\mathrm{X}($ end $))$;

TaperFin $=$ zeros $(1$, Index_FinTip -1$)$;

4

for i i $=1$ : Index_FinTip -1

$16 \quad \mathrm{Xtemp}=\operatorname{abs}(\mathrm{X}(\mathrm{i}$ i $)-\mathrm{X}(\mathrm{end}-\mathrm{i} \mathrm{i}+1))$;

$7 \quad$ TaperFin $($ i i $)=$ Xtemp/XRoot;

end

9

$\%$ Change Sweep

\%Calculates Fin along the Span, start at index of 2 because

$\mathrm{Xtemp}=\operatorname{abs}((\mathrm{X}(\mathrm{i}$ i $)+\mathrm{X}($ end $-\mathrm{i} \mathrm{i}+1)) / 2-\mathrm{XMin})$; 
$121 \%$ Changes the sweep of the fin while keeping the same taper distribution

${ }_{122} \%$ along the span.

${ }_{123}$ if sweep_flag $=$ true

124

SweepChange $=$ SweepNew/SweepCurrent; \%Ratio to multiply current sweep values

126

${ }_{127} \quad \%$ Pre-Allocate

128

129

130

131

132

133

134

135

for $\quad \mathrm{i} i=2:$ Index_FinTip -1

\%Need to reverse engineer the new outline of the fin SweepFin_New $($ i i -1$)=$ SweepFin $($ i i -1$) *$ SweepChange ;

SweepFin_Diff $($ ii -1$)=($ abs $($ SweepFin_New $($ i i -1$)-$ SweepFin $($ i i -1$)) / \ldots$

$(($ SweepFin_New $($ i i -1$)+$ SweepFin $($ i i -1$)) / 2)) * 100$; \% ok $<$ SAGROW 
147

165 166

167

\%Calculates New Shift in the X Midpoint, add XMin as that is the \%midpoint of the root chord Xtemp_New $=Y($ i i $) * \operatorname{tand}($ SweepFin_New $($ i i -1$))+$ XMin $;$ \%Add and subtract the taper at that location to get left and right $\%$ points XNew $($ ii $)=$ Xtemp_New + TaperFin $($ i i $) / 2$;

$\mathrm{XNew}($ end $-\mathrm{i} \mathrm{i}+1)=\mathrm{Xtemp} \_$New - TaperFin $(\mathrm{i}$ i $) / 2$;

YNew( i i $)=Y($ i i $)$;

YNew $($ end $-\mathrm{i} \mathrm{i}+1)=\mathrm{Y}($ end $-\mathrm{i} \mathrm{i}+1)$;

end

\%Include the new Vertex

YNew(Index_FinTip $)=$ Y(Index_FinTip $)$;

Xtemp $=\operatorname{tand}($ SweepNew $) * \mathrm{YNew}($ Index_FinTip $)$;

XNew(Index_FinTip) = Xtemp + XMin ;

end

\%\% Change Taper

\% Changes the taper of the fin while keeping the same sweep distribution

$\%$ along the span. if taper_flag $=$ true 


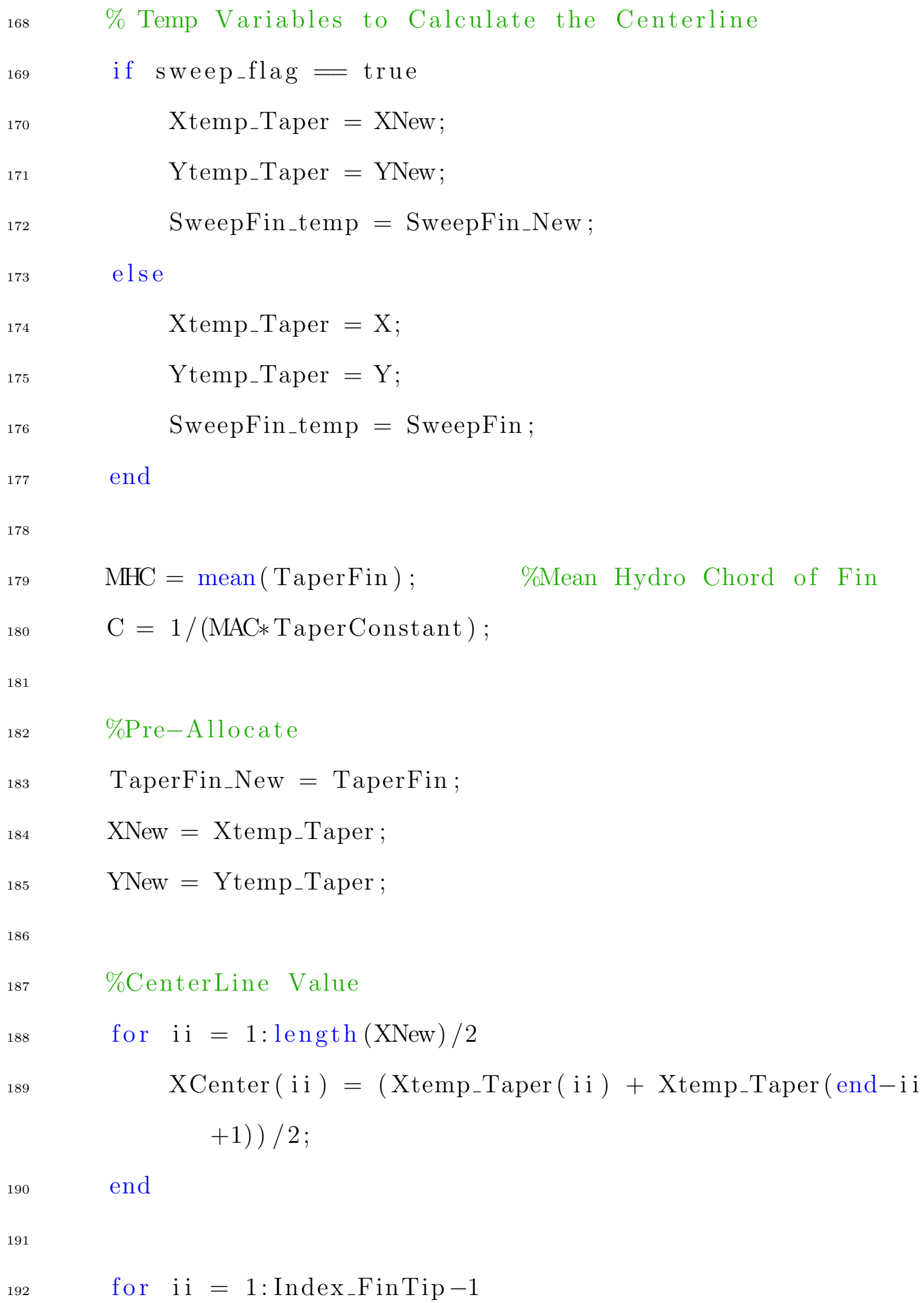

\% Temp Variables to Calculate the Centerline

if sweep_flag $=$ true

Xtemp_Taper $=$ XNew;

Ytemp_Taper $=$ YNew;

SweepFin_temp $=$ SweepFin_New ;

else

Xtemp_Taper $=\mathrm{X}$;

Ytemp_Taper $=\mathrm{Y}$;

SweepFin_temp $=$ SweepFin;

end

$\mathrm{MHC}=\operatorname{mean}($ TaperFin $) ; \quad$ \%ean Hydro Chord of Fin

$\mathrm{C}=1 /(\mathrm{MAC} *$ Taper Constant $)$;

\%Pre-Allocate

TaperFin_New $=$ TaperFin ;

XNew $=$ Xtemp_Taper ;

YNew $=$ Ytemp_Taper ;

\%CenterLine Value

$$
\begin{aligned}
& \text { for } \text { i i }=1: \operatorname{length}(\text { XNew }) / 2 \\
& \text { XCenter }(\text { i i })=\left(X_{\text {Xtemp_Taper }} \text { (i i }\right)+\text { Xtemp_Taper }(\text { end-i i }
\end{aligned}
$$$$
+1)) / 2 \text {; }
$$

end

for i i $=1:$ Index_FinTip -1 
195

198

206

$207 \%$ Split Lines

${ }_{208} \%$ Descrption: Splits the border into left and right lines for export

209

210

211

212 Index_Right $=$ Index_FinTip -1 ;

${ }_{213}$ Index_Left $=$ Index_FinTip +1 ;

214

215 if sweep_flag $_{-}$true || taper_flag $=$true 

orientation

224

225

226

227

228

229

230

231

232

233

234

235

236

(1)

Line_Right $=[$ XNew $(1$ : Index_Right $) '$, zeros (Index_Right, 1$)$, YNew (1:Index_Right)' ] ;

Line_Left $=[$ XNew(Index_Left $:$ end $) ', \quad$ zeros (Index_Right, 1$)$, YNew(Index_Left : end ) '] ;

else

\%Zero column in the middle to match solidworks orientation

Line_Right $=[X(1$ : Index_Right $) '$, zeros (Index_Right,1), Y (1:Index_Right)' ];

Line_Left $=\left[\mathrm{X}\left(\right.\right.$ Index_Left: end )', ${ }_{-}$zeros (Index_Right, 1$), \mathrm{Y}($ Index_Left : end ) '] ;

end

$\%$ CSV Write

if $\mathrm{CSV}_{-}$Write $=$true

\%Uncomment to write to csv

csvwrite ('Fin_Left.csv', Line_Left);

csvwrite ('Fin_Right.csv', Line_Right);

end

$\%$ Plot

figure (1)

clf

plot (X,Y, '. ', 'Color' ,' black', 'LineWidth', ,2) 
237 hold on

238

239 if sweep_flag $=$ true || taper_flag $=$ true

$240 \quad$ figure $(1)$

${ }_{241} \quad$ plot $(\mathrm{XNew}, \mathrm{YNew}$, '.g', 'LineWidth',2)

${ }_{242}$ legend('Fin Outline', 'New Fin Outline', 'Location', ' NorthWest')

243

244

Area $=\operatorname{abs}(\operatorname{trapz}(X, Y))$

245

Area_New $=\operatorname{abs}(\operatorname{trapz}(\mathrm{XNew}, \mathrm{YNew}))$

246

Percent_Difference_Area $=($ abs $($ Area-Area_New $) /(($ Area + Area_New ) /2) ) $* 100$

247 end 